volume 9

ISSUE 2

2021 agosto

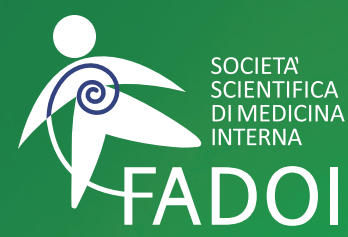

FEDERAZIONE

DELLE ASSOCIAZION

DEI DIRIGENTI

OSPEDALIERI

INTERNISTI

Editor in Chief

Michele Meschi

Supervisor Editor

Roberto Nardi
QUADERNI

\section{dell'Italian Journal} of Medicine

\section{A Journal of Hospital \\ and Internal Medicine}

The official journal of the Federation of Associations of Hospital Doctors on Internal Medicine (FADOI)

\title{
Lo scompenso cardiaco a 360 gradi
}

Guest Editors: Paola Gnerre, Giuseppe Di Pasquale 
PRESIDENTE ELETTO

Francesco Dentali, Varese, Italy

\section{PAST PRESIDENT}

Andrea Fontanella, Napoli, Italy

\section{SEGRETARIO}

Paola Gnerre, Savona, Italy

\section{STAFF DI SEGRETERIA}

Lorenza Lenzi, Pomarolo (TN), Italy

Ada Maffettone, Napoli, Italy

Claudia Tieri, Bari, Italy

\section{TESORIERE}

Giorgio Ballardini, Rimini, Italy

\section{COORDINATORE COMMISSIONE GIOVANI}

Ombretta Para, Firenze, Italy

\section{RESPONSABILE RAPPORTI ISTITUZIONALI}

Claudio Santini, Roma, Italy

\section{RESPONSABILE RAPPORTI CON LE REGIONI}

Alberto Fortini, Firenze, Italy

\section{RESPONSABILE EVENTI E INIZIATIVE SPECIALI}

Mauro Campanini, Novara, Italy

EDITOR IN CHIEF ITALIAN JOURNAL OF MEDICINE

Giorgio Vescovo, Padova, Italy
RESPONSABILE DEI

QUADERNI DELL'ITALIAN JOURNAL OF MEDICINE EDIZIONI ON LINE

Michele Meschi, Borgo Val di Taro (PR), Italy

SUPERVISOR EDITOR DEI

QUADERNI DELL'ITALIAN JOURNAL OF MEDICINE

Roberto Nardi, Bologna, Italy

\section{RESPONSABILE SITO WEB E COMUNICAZIONE SOCIAL}

Salvatore Lenti, Arezzo, Italy

Giuseppe Oteri, Milano, Italy

WEB MANAGER E CONTENT EDITOR

Giuseppe Oteri, Milano, Italy

Davide Ghilardi, Milano, Italy

\section{RESPONSABILE SISTEMA GESTIONE QUALITÀ}

Franco Berti, Roma, Italy

DPO

Alba Sciascera, Magenta (MI), Italy

\section{CONSULTA DEI PRESIDENTI}

Sandro Fontana, Biella, Italy

Salvatore Di Rosa, Palermo, Italy

Ido Iori, Reggio Emilia, Italy

Antonino Mazzone, Legnano (MI), Italy

Carlo Nozzoli, Firenze, Italy

Mauro Campanini, Novara, Italy

Andrea Fontanella, Napoli, Italy 


\section{Italian Journal of Medicine}

A Journal of Hospital and Internal Medicine

\section{COORDINATORE}

David Terracina, Roma, Italy

\section{SEGRETARIO E RESPONSABILE SCIENTIFICO} DEL PROVIDER ECM

Andrea Montagnani, Grosseto, Italy

DIRETTORI DEL DIPARTIMENTO

DELLA RICERCA CLINICA FADOI

Filippo Pieralli, Firenze, Italy

Fulvio Pomero, Savigliano (CN), Italy

\section{DIRETTORI DIPARTIMENTO}

\section{PER LA FORMAZIONE E AGGIORNAMENTO}

Luigi Magnani, Voghera (PV), Italy

Roberta Re, Novara, Italy

\section{COORDINATORE SCIENTIFICO CENTRO STUDI FONDAZIONE FADOI}

Gualberto Gussoni, Milano, Italy

\section{DELEGATI SOCIETÀ SCIENTIFICHE COLLEGATE}

FISM Antonino Mazzone, Legnano (MI), Italy

SIF Luigi Magnani, Voghera (PV), Italy

Consulta Michele Stornello, Siracusa, Italy cardiovascolare

SIIA e ISO

Michele Stornello, Siracusa, Italy Arcangelo Iannuzzi, Pomigliano d'Arco (NA), Italy

EFIM

Antonio Brucato, Milano, Italy Lorenza Lenzi, Pomarolo (TN), Italy

Choosing wisely Roberto Frediani, Chieri (TO), Italy

\section{PROGETTI SPECIALI}

\section{AGGIORN@FADOI}

Giuliano Pinna

PROGETTO NUOVE TECNOLOGIE

Francesco Nasso, Flavio Tangianu

PROGETTO MEDICINA DI GENERE

Cecilia Politi

PROGETTO COMPETENCE

Flavio Tangianu

PROGETTO GOVERNANCE

Stefano De Carli, Andrea Montagnani, Fabrizio Colombo

PROGETTO HOSPITALIST

Francesco Orlandini

PROGETTO FINE VITA

Mauro Carbone, Fabio Gilioli

PROGETTO GASTROENTEROLOGIA e FEGATO

Luca Fontanella, Paola Piccolo, Franco Radaelli, Giancarlo Parisi PROGETTO NUTRIZIONE CLINICA

Roberto Risicato, Luciano Tramontano

PROGETTO MALATTIE INFETTIVE/ANTIBIOTICI

Claudio Santini, Massimo Giusti, Marco Falcone

PROGETTO ECOGRAFIA INTERNISTICA

Francesco Cipollini, Nicola Mumoli

PROGETTO MALATTIE RARE

Antonio Brucato, Antonella Paradiso

PROGETTO BPCO/NIV

Marco Candela, Giuseppe De Matthaeis, Francesco Ventrella

PROGETTO TROMBOSI

Mauro Silingardi, Matteo Giorgi Pierfranceschi, Pierpaolo Di Micco PROGETTO TRIAL

Giancarlo Agnelli, Antonio Ceriello, Leo Fabbri, Claudio Ferri,

Franco Radaelli, Paolo Verdecchia

\section{PRESIDENTE ANÍMO}

Gabriella Bordin, Castelfranco Veneto (TV), Italy 


\title{
QUADERNI - Italian Journal of Medicine
}

\author{
LO SCOMPENSO CARDIACO A 360 GRADI
}

Guest Editor: Paola Gnerre, Giuseppe Di Pasquale

Presentazione

M. Meschi, P. Gnerre, A. Casola

Introduzione

G. Di Pasquale

TOPIC

Il paziente con scompenso cardiaco come paradigma del paziente complesso: il corretto uso dei diuretici ed il blocco sequenziale del nefrone.........

A. Cinque, C. Tarsia, A.G. Posteraro, S. Bianchi, A. Gaspardone

Scompenso cardiaco: differenze di genere?

R. Falzone, L. Lenzi, C. Politi

Opportunità terapeutiche nel paziente con scompenso cardiaco cronico a frazione d'eiezione depressa $\ldots \ldots \ldots \ldots \ldots \ldots \ldots \ldots \ldots \ldots$

A. Navazio, M. Piepoli, G.Q. Villani, G. Halasz, G. Tortorella

Opportunità terapeutiche nel paziente con scompenso cardiaco

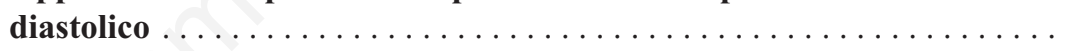

A. Cabassi, G. Regolisti

Scompenso cardiaco: rivalutazione della terapia

in fase di ospedalizzazione

F. Corradi, I. Lamberti, G. Gelati, S. Massini

I peptidi natriuretici nella diagnosi e nella stratificazione del rischio nei pazienti con scompenso cardiaco

A. Montagnani, L. Luschi, F. Pirrotta, A. Palazzuoli

L'educazione terapeutica nel paziente scompensato .

M.D. Corbo, E. Vitale, M. Correale, N.D. Brunetti, M. Iacoviello

Il modello del disease management nella gestione dello scompenso cardiaco .

O. Para

Modelli a confronto nei percorsi gestionali dello scompenso cardiaco: l'esperienza dell'AO Ordine Mauriziano di Torino

C. Norbiato, L. Arnaldi, S. Marengo, M. Tricarico, M. Daddea, C. Garza, E. Irene, P. Paolì

Modelli a confronto nei percorsi gestionali dello scompenso cardiaco: l'esperienza dell'ASST Rhodense $\ldots \ldots \ldots \ldots \ldots \ldots \ldots \ldots \ldots$ S.A. Berra 


\section{QUADERNI - Italian Journal of Medicine}

Modelli a confronto nei percorsi gestionali dello scompenso cardiaco:

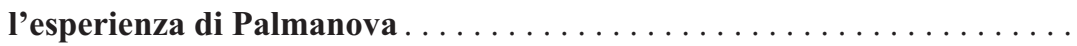

C. Battello

Modelli a confronto nei percorsi gestionali dello scompenso cardiaco:

l'esperienza dell'AUSL di Bologna .

S. Urbinati, M. Ongari

Modelli a confronto nei percorsi gestionali dello scompenso cardiaco: l'esperienza delle Aziende USL della Toscana . . . . . . . . . . . .

A. Fortini, G. Tintori, M. Alessandri

Modelli a confronto nei percorsi gestionali dello scompenso cardiaco:

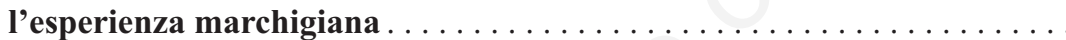
N. Tarquinio

Modelli a confronto nei percorsi gestionali dello scompenso cardiaco: l'esperienza savonese. Il progetto PONTE.

P. Gnerre, M. Pivari, E. Monaco, M.C. Pistone, M. Basso, P. Bellone, A. Visconti,

B. Sardo, B. Zanella, A.M. Saccone, A. Piras, A. Santo, F. Bernardi, S. Lapponia,

E. Montanari, R. Rapetti, R. Tassara, L. Parodi

Modelli a confronto nei percorsi gestionali dello scompenso cardiaco: l'esperienza campana $\ldots \ldots \ldots \ldots \ldots \ldots \ldots \ldots \ldots \ldots \ldots$

F. Gallucci

Modelli a confronto nei percorsi gestionali dello scompenso cardiaco:

l'esperienza pugliese. . . . . . . . . . . . . . . . .

F. Mastroianni, G. Larizza, F. D’Onofrio, A. Belfiore, M.V. Palma, S. Cataldi

Modelli a confronto nei percorsi gestionali dello scompenso cardiaco:

l'esperienza di Sassari. Il progetto LEAP

C.A. Usai, F.L. Bandiera

Il ruolo delle cure palliative nello scompenso cardiaco end-stage . . . . . . . 


\section{LO SCOMPENSO CARDIACO A 360 GRADI}

\section{Presentazione}

È con grande piacere che FADOI - Federazione Associazioni Dirigenti Ospedalieri Internisti, la Società Scientifica nata nel 1995 con l'intento di promuovere lo sviluppo delle conoscenze medico-scientifiche e della ricerca clinica nell'ambito della Medicina Interna, presenta una monografia della collezione Quaderni dell'Italian Journal of Medicine interamente dedicata allo scompenso cardiaco. L'insufficienza cardiaca è la patologia «exemplum» dell'interconnessione tra le comorbilità più frequenti nel pazienti internistici e problema sanitario sempre più diffuso, peraltro in significativo aumento in tutti gli ambiti di degenza ordinaria e di gestione ambulatoriale.

Le osservazioni epidemiologiche, nel nostro Paese come nella letteratura internazionale, ne evidenziano un impatto crescente sia nella popolazione generale sia, soprattutto, nelle fasce di età più avanzate. Le comorbilità coesistenti costituiscono un significativo fattore predittivo nel merito del numero di riospedalizzazioni per singolo paziente, che a loro volta divengono elementi determinanti nel computo definitivo dei costi assistenziali.

I modelli organizzativi proposti per ovviare parzialmente al problema sono molteplici e prevedono programmi di gestione integrata e multidisciplinare tra differenti professionisti della salute, sistemi di integrazione continua ospedale-territorio, esperienze innovative associate a nuove modalità comunicative (es. telemedicina).

Questo Quaderno è finalizzato ad un focus complessivo sui principali temi epidemiologici, fisiopatologici, terapeutici ed assistenziali che ruotano attorno al complesso universo dell'insufficienza cardiaca acuta e cronica. Frutto della collaborazione stretta tra le voci più autorevoli dell'esperienza della Medicina Interna, della Cardiologia, e di altre discipline specialistiche, tocca alcuni topics fondamentali e spesso non completamente chiariti, come il corretto impiego dei diuretici nello scompenso apparentemente refrattario, ed altri assolutamente inediti e innovativi, come l'impatto della differenza di genere sulla clinica di tali pazienti.

Tra gli aspetti trattati più eminentemente clinici, la gestione dello scompenso a frazione di eiezione ridotta e di quello a frazione di eiezione conservata, la rivalutazione della terapia in fase di ospedalizzazione, il significato pratico dell'utilizzo dei peptidi natriuretici e, in aggiunta, i fondamentali capitoli dell'educazione terapeutica del paziente e l'applicazione ad essa dei nuovi modelli di disease management.

La seconda parte è frutto, vivo ed attuale, della diretta esperienza di svariati centri italiani di trattamento dello scompenso cardiaco, nello sforzo di raccontare e confrontare i principali modelli di percorso gestionale nelle singole realtà di cura.

Infine, imprescindibile l'argomento delle cure palliative per lo scompenso cardiaco giunto allo stadio di insufficienza d'organo terminale, nella logica di un approccio che sta diventando sempre più cogente nella totalità degli ambiti della Medicina Interna.

A nome di FADOI il nostro più sentito ringraziamento a tutti coloro che hanno contribuito, con sapienza e dedizione, a questo impegnativo lavoro, in un anno - quello della pandemia da COVID-19 - tra i più difficili della nostra professione e della nostra parabola umana.

\author{
Michele Meschi \\ Direttore UOC Medicina Interna, \\ Presidio Ospedaliero \\ Azienda USL Parma, \\ Responsabile Quaderni \\ dell'Italian Journal of Medicine
}

\author{
Paola Gnerre \\ Direttore UOC Medicina Generale, \\ Ospedale "Monsignor Galliano" \\ Acqui Terme, \\ Segretario Nazionale FADOI
}

\author{
Alessia Casola \\ UOC Medicina Interna, \\ Presidio Ospedaliero \\ Azienda USL Parma, \\ Responsabile Commissione Giovani \\ FADOI Regione Emilia-Romagna
}




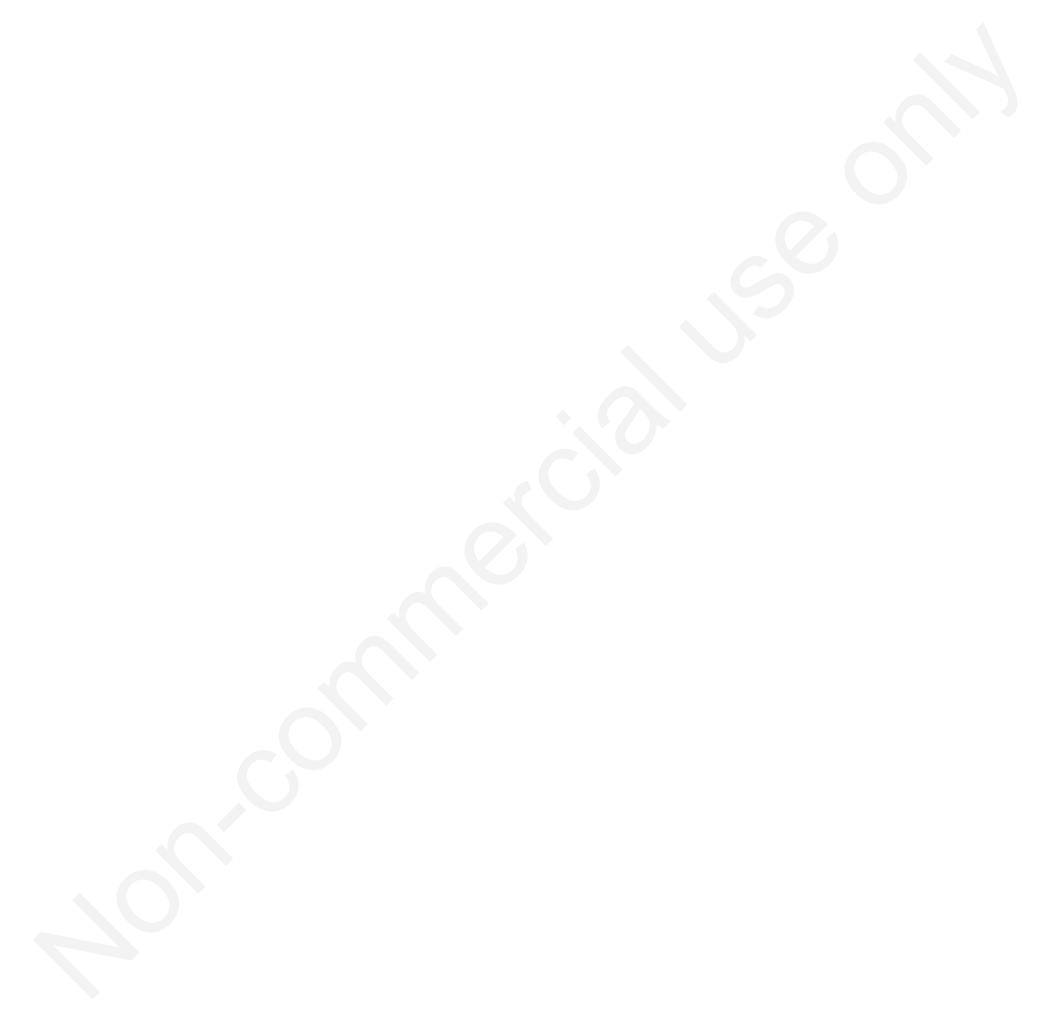




\title{
Lo scompenso cardiaco a 360 gradi
}

\author{
Giuseppe Di Pasquale \\ Consulente Direzione Sanitaria, Azienda USL di Bologna, Italia
}

\section{Introduzione}

La prognosi dei pazienti con scompenso cardiaco (SC) è notevolmente migliorata rispetto al passato grazie agli avanzamenti terapeutici e la diffusione, anche se piuttosto disomogenea e con modalità diverse, di modelli di disease management ospedale-territorio. Tutto questo riguarda i pazienti con SC cronico, in particolare quelli con ridotta frazione di eiezione ventricolare sinistra (HFrEF). Per quanto riguarda invece lo SC acuto gli ultimi decenni sono stati caratterizzati da aspettative deluse per nuovi approcci terapeutici (nesiritide, tolvaptan, rolofillina, serelaxina) e la prognosi è rimasta sostanzialmente invariata da tre decadi. ${ }^{1}$ Analogamente, per quanto riguarda lo SC con frazione di eiezione preservata (HFpEF) che è la forma prevalente nel setting della Medicina Interna tutti i farmaci finora testati nei trial clinici (ACE inibitori, sartani, $\beta$-bloccanti, antagonisti mineralcorticoidi, ARNI) hanno dato risultati deludenti. ${ }^{2}$ Questo verosimilmente in relazione all'eterogeneità dei fenotipi fisiopatologici presenti all'interno della sindrome multiforme HFpEF che rimane una diagnosi difficile, formulata spesso per esclusione, con i limiti degli algoritmi diagnostici probabilistici che sono stati proposti, quali il $\mathrm{H}_{2}$ FPEF score ${ }^{3}$ ed il HFA-PEFF score. ${ }^{4}$

\section{Obiettivi del trattamento}

Gli obiettivi del trattamento dei pazienti con SC sono la riduzione della mortalità e delle (re)-ospedalizzazioni. Questo costituisce l'endpoint composito primario richiesto anche dalle autorità regolatorie per

Corrispondente: Giuseppe Di Pasquale, Consulente Direzione Sanitaria, Azienda USL di Bologna, Italia.

E-mail: g.dipa@libero.it

Articolo pubblicato secondo la Creative Commons Attribution NonCommercial 4.0 License (CC BY-NC 4.0).

${ }^{\circ}$ Copyright: the Author(s), 2021

Licensee PAGEPress, Italy

QUADERNI - Italian Journal of Medicine 2021; 9(2):e1 l'approvazione di nuovi farmaci per lo SC. La riduzione delle ospedalizzazioni per SC ha un impatto significativo non solo sulla qualità di vita dei pazienti ma anche sulla prognosi a medio-lungo termine. Il paziente ricoverato per SC acuto, una volta dimesso dall'ospedale non è più lo stesso di prima, diventando molto più vulnerabile. L'obiettivo da perseguire è quello di ridurre le re-ospedalizzazioni per SC ma anche quelle per altre cause cardiologiche e non cardiologiche che costituiscono fino al 50\% delle cause di re-ospedalizzazione. Lo studio TEMISTOCLE condotto nel 2000 da ANMCO e FADOI ha dimostrato che il rate di re-ospedalizzazione dalla dimissione a sei mesi era del tutto sovrapponibile per i pazienti dimessi da reparti di Cardiologia e di Medicina Interna $(43,7 \%$ vs $45,4 \%) .{ }^{5}$ Più recentemente i dati di mondo reale provenienti dall'Osservatorio ARNO in una casistica di 41.413 pazienti con SC dimessi vivi hanno dimostrato che a distanza di dodici mesi dalla dimissione per SC almeno una re-ospedalizzazione si è verificata nel 56\% dei pazienti, nella metà dei casi per cause non cardiovascolari (esacerbazione di broncopneumopatia cronica ostruttiva (BPCO), infezioni polmonari, insufficienza renale, cancro, ecc.). ${ }^{6}$

Risulta pertanto evidente che l'approccio al paziente con SC deve essere omnicomprensivo, finalizzato a trattare anche le patologie associate. Nel 74\% dei pazienti con SC è presente infatti almeno una comorbilità (malattia renale $41 \%$, anemia $29 \%$, diabete $29 \%$, BPCO $15 \%$, stroke $11 \%$ ) ed il numero di comorbilità aumenta con la severità dello SC. ${ }^{7}$ Le comorbilità hanno un impatto importante sulla qualità di vita e la prognosi, determinando una quota significativa della mortalità per tutte le cause dei pazienti con SC. Un approccio olistico al paziente con SC è connotato al DNA dell'internista, mentre il cardiologo è di solito prevalentemente focalizzato sul cuore.

\section{Dalla triplice alla quadruplice terapia e oltre}

La terapia convenzionale dello SC fino a pochi anni fa era costituita dagli ACE inibitori o sartani, $\beta$ bloccanti e antialdosteronici. La prima rivoluzione terapeutica è avvenuta nel 2014 con la pubblicazione dello studio PARADIGM-HF che ha dimostrato che 
il sacubitril valsartan, appartenente ad una nuova classe di farmaci denominati ARNI, era in grado di ridurre rispetto all'enalapril del $20 \%$ l'endpoint composito di morte cardiovascolare e ospedalizzazioni per SC con un beneficio significativo anche per la mortalità. ${ }^{8}$ Più recentemente la nuova classe di farmaci antidiabetici inibitori SGLT2, in particolare dapaglifozin ed empaglifozin in aggiunta alla terapia standard ottimizzata, hanno dimostrato di essere in grado di ridurre l'endpoint composito di morte e ospedalizzazione per SC nei pazienti HFrEF anche in assenza di diabete, proponendosi come il quarto pilastro nella terapia dello SC. ${ }^{9,10}$ Purtroppo l'uptake del sacubitril valsartan è ancora modesto, in parte per le limitazioni imposte dal piano terapeutico che in accordo con le linee guida ESC 2016 sullo SC un po' corrose dal tempo richiede che il farmaco possa essere prescritto se il paziente con SC è ancora sintomatico dopo un iniziale trattamento con ACE inibitori o sartani, ed in parte anche per una reale inerzia terapeutica che ampiamente condividiamo con i colleghi di altri Paesi. ${ }^{11}$ Per l'utilizzo degli inibitori SGLT2 nei pazienti con HFrEF con e senza diabete mellito nel novembre 2020 è arrivata l'autorizzazione dell'EMA per il dapaglifozin, mentre siamo ancora in attesa della rimborsabilità da parte di AIFA, ma purtroppo anche nei pazienti diabetici l'utilizzo di questa classe di farmaci è ancora modesto.

Un'analisi recente pubblicata da Vaduganathan et $a l .{ }^{12}$ ha dimostrato utilizzando i dati provenienti da tre trial clinici con evidenza di beneficio significativo sulla mortalità (EMPHASIS-HF con eplerenone, ${ }^{13}$ PARADIGM-HF con sacubitril valsartan ${ }^{8}$ e DAPA-HF con dapaglifozin ${ }^{9}$ ) che un trattamento con tutte e quattro le classi di farmaci (ARNI, $\beta$-bloccanti, antagonisti mineralcorticolidi e inibitori SGLT2) produce un guadagno di sopravvivenza di 6,3 anni in un paziente con HFrEF di 55 anni e di 1,4 anni in un paziente di 80 anni, ed un beneficio rispettivo di 8,3 e 2,7 anni in termini di morte o ospedalizzazione per SC, rispetto alla terapia medica convenzionale costituita da ACE inibitori o sartani e $\beta$ blocacanti. Passando dalla teoria alla pratica rimane un interrogativo non risolto se una quadruplice terapia vada iniziata subito oppure procedendo per step dopo avere cercato di titolare i singoli farmaci alle dosi raccomandate dalle linee guida. ${ }^{14}$

In aggiunta a queste quattro classi di farmaci che sono in grado di modificare la prognosi dello SC, esistono da anni ampie evidenze sull'efficacia del ferro carbomaltoso per la correzione della carenza di ferro che è frequente nei pazienti con SC. I trial clinici randomizzati, compreso l'AFFIRM-AHF recentemente pubblicato ${ }^{15}$ hanno dimostrato una sicura efficacia di questo trattamento nella riduzione delle ospedalizzazioni, senza un apparente effetto sulla mortalità. Infine per la prima volta un farmaco che agisce sull'inotro- pismo, omecamtiv-mecarbil attivatore selettivo della miosina cardiaca, ha dimostrato l'efficacia nella riduzione degli eventi di scompenso in pazienti con SC cronico, anche se i benefici sono risultati complessivamente modesti. ${ }^{16}$ È difficile intravedere quale futuro potrà esserci per questo farmaco.

\section{I trattamenti non farmacologici}

I trattamenti non farmacologici sono anzitutto rappresentati da interventi finalizzati alla correzione delle cause eziologiche dello SC che comprendono rivascolarizzazione miocardica mediante PTCA o bypass aorto-coronarico, correzione delle valvulopatie aortica e mitralica mediante approccio cardiochirurgico o interventistico percutaneo (TAVI e mitraclip) e ablazione transcatetere della fibrillazione atriale.

Una volta escluse eziologie cardiache potenzialmente trattabili alla base dello SC vanno considerate le indicazioni al defibrillatore impiantabile (ICD) per la prevenzione della morte improvvisa nei pazienti con FE ridotta nonostante terapia medica ottimale e la resincronizzazione cardiaca (CRT) che nei pazienti con blocco di branca sinistra è in grado di migliorare la funzione cardiaca, la qualità di vita e ridurre le ospedalizzazioni in pazienti con $\mathrm{FE}<35 \%$ e QRS largo (>130 ms) con evidenza di blocco di branca sinistra.

Nei pazienti non candidabili alla CRT sta emergendo la possibilità di una nuova opzione terapeutica rappresentata dalla modulazione della contrattilità cardiaca (CCM) ${ }^{17}$ La CCM si basa su un sistema di trasmissione di un impulso elettrico non eccitatorio a livello del setto interventricolare determinando un miglioramento della performance cardiaca. Il dispositivo impiantabile per la CCM è simile ad un pacemaker con un elettrodo posizionato nell'atrio destro e gli altri due nel ventricolo destro. Gli studi clinici finora eseguiti dimostrano che la CCM è sicura ed efficace nel ridurre i ricoveri per SC e nel migliorare i sintomi. Sono tuttavia necessari ulteriori studi su casistiche più ampie e con più lungo follow-up prima che questo presidio elettrico possa trovare spazio nelle raccomandazioni delle linee guida.

Dal momento che fino all' $80 \%$ dei pazienti con $\mathrm{SC}$ sono ricoverati nei reparti di Medicina Interna è necessario che l'internista individui i pazienti che meritano di essere riferiti al cardiologo per valutare le indicazioni ad una diagnostica cardiologica di secondo livello oppure a trattamenti cardiochirurgici o interventistici percutanei oppure a terapie elettriche. Una valutazione cardiologica durante il ricovero è particolarmente appropriata per i pazienti con SC de novo ed in quelli con HFrEF. In questi casi una gestione integrata internista-cardiologo è fortemente auspicabile. 


\section{Disease management ospedale-territorio: una strategia a tre fasi}

A metà degli anni ' 90 sono state prodotte le prime evidenze che un intervento multidisciplinare, comprensivo di un coinvolgimento infermieristico, con la gestione del paziente a domicilio dopo la dimissione dall'ospedale era in grado di ridurre significativamente le re-ospedalizzazioni nei soggetti anziani con SC. ${ }^{18}$ I modelli di disease management ospedale-territorio sono numerosi ed in questa monografia vengono messe a confronto diverse esperienze, compresa la nostra in atto da anni nell'Azienda USL di Bologna. In diversi di questi modelli è prevista un'analisi di indicatori di processo e di outcome, ricavabili dai database amministrativi ed eventualmente dalle lettere di dimissione e visite ambulatoriali informatizzate, per valutare l'efficacia degli interventi.

Una prima fase particolarmente importante di questi programmi, che recentemente ha ricevuto considerevole attenzione in letteratura, è la cosiddetta fase di transizione dalla dimissione dall'ospedale al rientro a domicilio. È noto infatti che esiste un cluster di reospedalizzazioni precoci a 30 giorni stimato intorno al $23-27 \%$, con un $17 \%$ entro quindici giorni ad un tempo mediano di 12 giorni. ${ }^{19}$ È pertanto fondamentale una presa in carico precoce del paziente dopo la dimissione e particolarmente importante è il follow-up entro 7-10 giorni, prevedendo un controllo ambulatoriale dopo breve tempo dalla dimissione. . $^{19,20}$

Successivamente, nella cosiddetta seconda fase di plateau è necessaria una continua vigilanza sul territorio per evitare la destabilizzazione del compenso, prestando particolare attenzione all'ottimizzazione della terapia e all'aderenza terapeutica, insieme ad un attento monitoraggio per il riconoscimento dei segni precoci di peggioramento della congestione. L'utilizzo della telemedicina nei primi studi pubblicati negli anni 2010 e 2011 ha dato risultati deludenti non riuscendo a dimostrare una riduzione di mortalità e reospedalizzazioni. ${ }^{21,22}$ Le esperienze italiane, principalmente condotte nei centri della Fondazione "S. Maugeri", in regione Lombardia e a Trieste hanno invece dimostrato che la telemedicina può portare benefici al paziente con SC se viene inserita in un modello di gestione ospedale-territorio condivisa e integrata che costituisce il "Chronic Care Model" nazionale. ${ }^{23}$

La terza fase è quella delle cure palliative che subentrano nelle fasi finali della vita del paziente con SC quando la prognosi non è più il criterio principale per definire le scelte, ma prevalgono come fattori di riferimento la soddisfazione dei bisogni del paziente e dei famigliari, bisogni clinici, psicologici, spirituali e di condivisione ${ }^{24} \mathrm{La}$ cultura delle cure palliative in ambito cardiologico non è tuttavia ancora diffusa ed esi- ste la necessità di strumenti informativi e opportune modalità organizzative..$^{25}$

\section{Conclusioni}

Lo SC più di ogni altra malattia richiede una visione a 360 gradi, dal momento che il paziente con $\mathrm{SC}$ è forse il prototipo del paziente complesso e della cronicità. ${ }^{23}$ Avere una visione a 360 gradi significa non concentrarsi soltanto sulla patologia cardiaca, ma anche sulle condizioni morbose coesistenti. Questo vale in particolare per il paziente HFpEF ampiamente rappresentato nel setting della Medicina Interna. In ogni realtà dovrebbe essere implementata la collaborazione tra la Medicina Interna e la Cardiologia per l'identificazione dei pazienti, in modo particolare quelli meno anziani, con SC de novo o HFrEF per i quali è appropriato uno specifico percorso cardiologico. L'aderenza alle linee guida è di fondamentale importanza ed ha un impatto sull'outcome, anche se i pazienti con SC del real world sono sostanzialmente diversi da quelli sui quali sono state costruite le linee guida. Avere una visione a 360 gradi significa non limitarsi alla gestione ottimale durante il ricovero in ospedale, ma assicurare al paziente con SC una continuità assistenziale sul territorio attraverso la collaborazione tra assistenza specialistica, medicina generale e assistenza infermieristica in una rete integrata che preveda percorsi condivisi e comunicazione.

\section{Bibliografia}

1. Tavazzi L. Progressi nella gestione dello scompenso cardiaco. G Ital Cardiol 2021;22:3-19.

2. Ferrari R, Bohm M, Cleland GF et al. Heart failure with preserved ejection fraction: uncertainties and dilemmas. Eur J Heart Fail 2015; 17:665-71.

3. Reddy YNV, Carter RE, Obokata M, Redfield MM, Borlaug BA. A simple, evidence-based approach to help guide diagnosis of heart failure with preserved ejection fraction. Circulation 2018;138:861-70.

4. Pieske B, Tschope C, de Boer RA et al. How to diagnose heart failure with preserved ejection fraction: the HFAPEFF diagnostic algorithm. A consensus recommendation from the Heart Failure Association (HFA) of the European Society of Cardiology (ESC). Eur Heart J 2019;40:3297-317.

5. Di Lenarda A, Scherillo M, Maggioni AP, et al. Current presentation and management of heart failure in cardiology and internal medicine hospital units: A tale of two worlds The TEMISTOCLE study. Am Heart J 2000;146:e12.

6. Maggioni AP, Orso F, Calabria S, et al; on behalf of the ARNO Observatory. The real-world evidence of heart failure: findings from 41.413 patientes of the ARNO database. Eur J Heart Fail 2016;18:402-10.

7. Van Deursen VM, Urso R, Laroche C, et al. Co-morbidities in patients with heart failure: an analysis of the 
European Heart Failure Pilot Survey. Eur J Heart Fail 2014;16:103-11.

8. McMurray JJV, Packer M, Desai AS, et al.; for the PARADIGM-HF Investigators and Committees. Angiotensin-neprilysin inhibition versus enalapril in heart failure. N Engl J Med 2014;371:993-1004.

9. McMurray JJV, Solomon SD, Inzucchi SE, et al. for the DAPA-HF Trial Committes and Investigators. Dapaglifozin in patients with heart failure and reduced ejection fraction. N Engl J Med 2019;381:1995-2008.

10. Packer M, Anker SD, Butler J et al.; for the EMPERORReduced Trial Investigators. Cardiovascular and renal outcomes with empaglifozin in heart failure. N Engl $\mathbf{J}$ Med 2020;383:1413-24.

11. Ponikowski P, Voors AA, Anker SD, et al. 2016 ESC Guidelines for the diagnosis and treatment of acute and chronic heart failure. Eur Heart J 2016;37:2129-200.

12. Vaduganathan M, Clagget BL, Jhund PS, et al. Estimating lifetime benefits of comprehensive disease-modifying pharmacological therapies in patients with heart failure with reduced ejection fraction: a comparative analysis of three randomised controlled trials. Lancet 2020;396:121-8.

13. Zannad F, McMurray JJV, Krum H, et al. Eplerenone in patients with systolic heart failure and mild symptoms. N Engl J Med 2001;364:11-21.

14. McMurray JJV, Packer M. How should we sequence the treatment for heart failure and a reduced ejection fraction? A redefinition of evidence-based medicine. Circulation 2021;143:875-877.

15. Ponikowski P, Kirwan B-A, Anker SD, et al.; on behalf of the AFFIRM-AHF Investigators. Ferric carboxymaltose for iron deficiency at discharge after acute heart failure: a multicentre, double-blind, randomised, controlled trial. Lancet 2020;396:1895-1904.

16. Teerlink JR, Diaz R, Felker GM, et al.; for the GALACTIC-HF Investigators. Cardiac myosin activation with omecamtiv mecarbil in systolic heart failure. N Engl J Med 2021;384:105-116.
17. Cappannoli L, Scacciavillani R, Rocco E, et al. La modulazione della contrattilità cardiaca: un'opzione terapeutica per il paziente con insufficienza cardiaca refrattaria. G Ital Cardiol 2021;22:212-220.

18. Rich MW, Beckham V, Wittenberg C, et al. A multidisciplinary intervention to prevent the readmission of elderly patients with congestive heart failure. N Engl J Med 1995;333:1190-5.

19. Desai AS, Stevenson LW. Rehospitalization for heart failure. Predict or prevent? Circulation 2012;126:501-6.

20. Di Lenarda, Colivicchi F, Gabrielli D. L'ospedalizzazione per scompenso cardiaco acuto: come un evento critico può diventare un'opportunità clinica. G Ital Cardol 2019;20:690-3.

21. Choudry SI, Mattera JA, Curtis JP, et al. Telemonitoring in patients with heart failure. $\mathrm{N}$ Engl $\mathrm{J}$ Med 2010;363:2301-9.

22. Koehler F, Winkler S, Schieber M, et al. Impact of remote telemedical management on mortality and hospitalizations in ambulatory patients with chronic heart failure: The Telemedical Interventional Monitoring in Heart Failure study: Circulation 2011;123:1873-80.

23. Di Lenarda A, Casolo G, Gulizia MM et al. Documento di consenso ANMCO/SIC/SIT: il futuro della telemedicina nello scompenso cardiaco. G Ital Cardiol 2016;17:491-507.

24. Sinagra G, Romanò M, Antonione R. Indicazioni pratiche per un'efficace implementazione delle cure palliative nel malato con scompenso cardiaco. G Ital Cardiol 2020;21:271-308.

25. Antonione R, Sinagra G, Moroni M, et al. Documento di consenso sulle cure palliative in ambito cardiologico a cura del Gruppo di Lavoro congiunto della Società Italiana di Cardiologia (SIC) e della Società Italiana di Cure Palliative (SICP). G Ital Cardiol 2019;20:46-61.

26. Allegri M, Bevere F, Boccanelli A, et al. Criteri di appropriatezza clinica, tecnologica e strutturale nell'assistenza al paziente complesso. Quaderni del Ministero della Salute N. 23, settembre-ottobre 2013. 


\title{
Il paziente con scompenso cardiaco come paradigma del paziente complesso: il corretto uso dei diuretici ed il blocco sequenziale del nefrone
}

\author{
Alessandra Cinque, ${ }^{1}$ Carmela Tarsia, ${ }^{1}$ Antonio Giuseppe Posteraro, ${ }^{1}$ Stefano Bianchi, ${ }^{2}$ Achille Gaspardone ${ }^{1}$ \\ ${ }^{1}$ U.O.C. di Cardiologia, Ospedale Sant'Eugenio, Roma; ${ }^{2}$ U.O.C di Nefrologia e Dialisi, Zona Livornese, ASL Toscana Nordovest, \\ Livorno, Italia
}

\section{Introduzione}

La storia naturale dello scompenso cardiaco è caratterizzata da episodi di riacutizzazione e ospedalizzazione che peggiorano la prognosi del paziente e la cui causa più frequente è il peggioramento dei sintomi legati alla congestione, mentre soltanto una minoranza dei pazienti si presenta acutamente con segni e sintomi da ipoperfusione. ${ }^{1}$

La congestione consiste nell'accumulo di liquidi nel comparto intravascolare e nello spazio interstiziale. Di fatto si distinguono due forme congestizie, intravascolare e tissutale, variabilmente presenti in ciascun paziente. Sebbene nella maggior parte dei casi siano presenti entrambe le forme, è tuttavia possibile identificare un fenotipo predominante in ciascun caso e l'importanza di distinguerlo consiste in un differente approccio terapeutico.

La congestione intravascolare è il risultato della ritenzione renale di sodio e acqua causata da una serie di fattori emodinamici, e non, che si realizzato nello scompenso cardiaco: la ridotta gittata cardiaca, l'attivazione del sistema renina-angiotensina-aldosterone, dell'asse dei peptidi netriuretici e del sistema nervoso simpatico. ${ }^{2}$ Oltre all'incremento assoluto della volemia, si realizza anche uno spostamento di sangue dal sistema venoso splancnico nel torrente circolatorio centrale. Le vene splancniche contengono normalmente il $20-50 \%$ della volemia totale, fungendo per-

Corrispondente: Alessandra Cinque, U.O.C. di Cardiologia, Ospedale Sant'Eugenio, Piazzale dell'Umanesimo 10, 00144 Roma, Italia.

E-mail: cinquealessandra@gmail.com

Articolo pubblicato secondo la Creative Commons Attribution NonCommercial 4.0 License (CC BY-NC 4.0).

${ }^{\circ}$ Copyright: the Author(s), 2021

Licensee PAGEPress, Italy

QUADERNI - Italian Journal of Medicine 2021; 9(2):e2 tanto da reservoir ematico, e sono ricche di recettori alfa-adrenergici che rispondono all'iperattivazione del sistema nervoso simpatico in corso di scompenso con conseguente vasocostrizione e shift volemico. ${ }^{3}$

La congestione tissutale è il risultato dello squilibrio di pressioni tra il letto capillare e l'interstizio. Generalmente nello scompenso l'edema tissutale è secondario alla congestione intravascolare. Tuttavia in alcuni pazienti è possibile osservare una predominante congestione tissutale, in assenza di una altrettanto significativa congestione intravascolare. La causa può risiedere nella perdita dell'integrità strutturale dell'interstizio che, con la sua rete di glicosamminoglicani, sembra avere un ruolo nell'omeostasi del sodio e contribuirebbe perciò a proteggere dall'ipernatremia che si realizza nello scompenso. ${ }^{4}$ Tuttavia l'eccesso di sodio nell'interstizio può alterarne la struttura, facendo sì che anche un piccolo incremento della pressione idrostatica capillare causi edema. ${ }^{5}$ Un altro possibile fattore causale può risiedere nella ridotta permeabilità vascolare, tipica ad es. della sepsi o del danno vascolare nel diabetico.

Per un adeguato indirizzo terapeutico occorre una corretta identificazione e quantificazione del grado di congestione intravascolare. ${ }^{6}$ Il gold standard è il cateterismo cardiaco destro, con misurazione della pressione atriale destra (v.n. 2-6 mmHg) e della wedge pressure (v.n. 3-8 mmHg). Tuttavia l'invasività della procedura ne limita l'utilizzo e la valutazione clinica routinaria si realizza di fatto mediante la raccolta di sintomi e segni, quali la dispnea, l'ortopnea, la distensione delle vene giugulari, l'auscultazione del terzo tono, l'incremento dei peptidi natriuretici. È di ausilio l'ecocardiografia con la valutazione del diametro e della collassabilità della vena cava inferiore: una collassabilità inspiratoria $<50 \%$ è segno di una pressione atriale destra $\geq 10 \mathrm{mmHg}$. Sono segni di congestione tissutale l'edema degli arti inferiori, l'ascite, i rumori polmonari all'auscultazione toracica, nonché il riscontro di edema polmonare alla radiografia del torace o all'ecografia polmonare. 
I diuretici agiscono sulla congestione intravascolare perché riducono l'ipervolemia che ne è la causa mediante meccanismi di natriuresi (riduzione del riassorbimento tubulare di sodio e quindi di acqua) o acquaresi diretta (riduzione del riassorbimento tubulare di acqua con riduzione dell'osmolarità urinaria, aumento di quella plasmatica e conseguente traslocazione di liquidi dall'interstizio al letto vascolare).

\section{Criteri e strumenti di ricerca}

Partendo dalle indicazioni delle linee guida internazionali sull'utilizzo dei diuretici nello scompenso cardiaco, abbiamo analizzato le più rilevanti pubblicazioni scientifiche presenti in PubMed e consultato i testi di farmacologia clinica per un adeguato ripasso dei meccanismi di azione dei farmaci argomento di tale monografia.

\section{I farmaci diuretici: evidenze e meccanismi di azione}

Nonostante nessun trial clinico randomizzato abbia dimostrato la loro efficacia nella riduzione della mortalità, i farmaci diuretici determinano un miglioramento dei sintomi più rapidamente rispetto a qualsiasi altro farmaco per lo scompenso; le linee guida internazionali ne raccomandano pertanto l'utilizzo come terapia di prima scelta. ${ }^{7,8}$

I diuretici dell'ansa sono sicuramente i più utilizzati. Tuttavia nelle fasi avanzate dello scompenso l'efficacia di questa classe di farmaci tende a ridursi ed è necessario sfruttare l'effetto sinergico delle diverse classi di diuretici che agiscono in punti diversi nel nefrone. Di fatto, il riassorbimento di acqua ed elettroliti filtrati a livello glomerulare si verifica in più punti $\mathrm{e}$ secondo diverse modalità nella porzione tubulare del nefrone. Ognuno di tali siti rappresenta un possibile bersaglio dei diuretici.

La conoscenza della farmacodinamica dei farmaci diuretici è fondamentale per sfruttare l'effetto sinergico delle diverse classi (Figura 1).

\section{Diuretici dell'ansa}

Questa classe di farmaci inibisce il riassorbimento di cloruro di sodio $(\mathrm{NaCl})$ nel tratto spesso ascendente dell'ansa di Henle, agendo sul trasportatore $\mathrm{Na}^{+} / \mathrm{K}^{+} / 2 \mathrm{Cl}$, denominato anche $\mathrm{NKCC}_{2}$ o $\mathrm{NK}_{2} \mathrm{CL}$. Seppure questo trasportatore sia di per sé elettricamente neutro, la sua azione provoca un accumulo di $\mathrm{K}^{+}$nelle cellule e una retrodiffusione dello stesso ione nel lume tubulare; il potenziale elettrico che ne deriva fornisce l'energia per il riassorbimento di cationi bivalenti, compresi magnesio $\left(\mathrm{Mg}_{2}^{+}\right)$e calcio $\left(\mathrm{Ca}_{2}^{+}\right)$, tra le cellule. Riducendo questo meccanismo, i diuretici dell'ansa causano un aumento dell'escrezione di $\mathrm{Mg}_{2}{ }^{+}$ e $\mathrm{Ca}_{2}{ }^{+}$. Questa classe di farmaci induce la sintesi di prostaglandine renali con un effetto diretto sul flusso ematico in diversi distretti vascolari, oltre quello renale, riducendo la congestione polmonare e le pressioni di riempimento del ventricolo sinistro nell'insufficienza cardiaca, ancor prima che si manifesti il loro principale effetto diuretico.

\section{Inibitori dell'anidrasi carbonica}

L'anidrasi carbonica è presente in molti siti del nefrone, ma il più importante si trova nelle membrane luminali delle cellule del tubulo prossimale. Il riassorbimento del bicarbonato di sodio $\left(\mathrm{HCO}_{3}^{-}\right)$inizia per l'azione di uno scambiatore $\mathrm{Na}^{+} / \mathrm{H}^{+}$che permette il passaggio del $\mathrm{Na}^{+}$presente nel lume tubulare all'interno della cellula scambiando uno ione $\mathrm{H}^{+}$che passa all'esterno della cellula. Gli ioni $\mathrm{H}^{+}$reagiscono con l'anione $\mathrm{HCO}_{3}{ }^{-}$formando acido carbonico $\left(\mathrm{H}_{2} \mathrm{CO}_{3}\right)$ che viene rapidamente deidratato dall'anidrasi carbonica $(\mathrm{CAI})$ ad anidride carbonica $\left(\mathrm{CO}_{2}\right)$ ed acqua $\left(\mathrm{H}_{2} \mathrm{O}\right)$ che a loro volta entrano nella cellula per diffusione. L'enzima anidrasi carbonica intracellulare riconverte l' $\mathrm{H}_{2} \mathrm{O}$ e la $\mathrm{CO}_{2}$ a $\mathrm{H}_{2} \mathrm{CO}_{3}$ che rapidamente si dissocia in $\mathrm{H}^{+}$e $\mathrm{HCO}_{3}^{-}$. Lo ione $\mathrm{H}^{+}$sarà quindi disponibile per lo scambiatore $\mathrm{Na}^{+} / \mathrm{H}^{+}$e l' $\mathrm{HCO}_{3}^{-}$viene trasportato fuori dalla cellula mediante un trasportatore presente sulla membrana cellulare baso-laterale. Inibendo l'enzima, tali farmaci causano una riduzione del riassorbimento del bicarbonato da parte delle cellule tubulari, determinando l'alcalinizzazione delle urine e l'acidificazione del sangue. L'effetto diuretico che ne consegue provoca una maggiore escrezione di acqua e una diminuzione della pressione sanguigna. L'anidrasi carbonica è presente anche in siti extrarenali, tra cui gli occhi dove è coinvolta nella produzione dell'umor acqueo da parte dell'epitelio del corpo ciliare. La ridotta produzione di bicarbonato, e di conseguenza dell'umor acqueo, abbassa la pressione

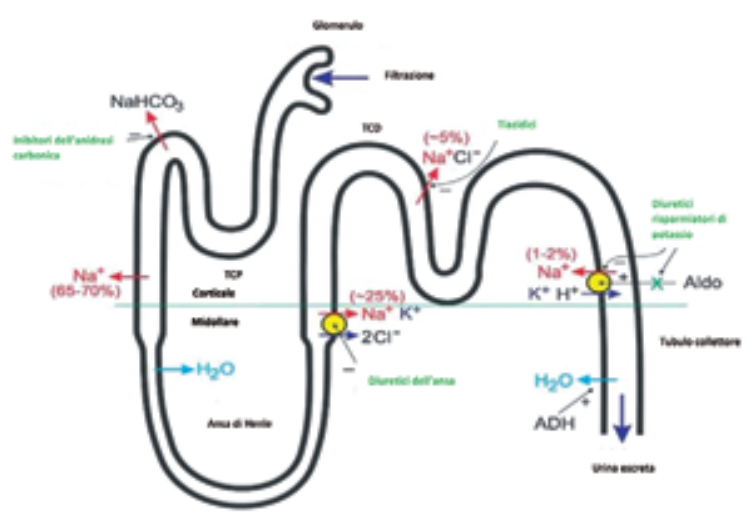

Figura 1. Siti di azione dei farmaci diuretici (TCP, tubulo contorto prossimale; TCD, tubulo contorto distale). 
intraoculare, rendendo questi farmaci utili nei pazienti con glaucoma.

\section{Tiazidici}

Nati dalla necessità di ottenere inibitori dell'anidrasi carbonica più potenti, è successiva la scoperta che il meccanismo d'azione di questa classe di farmaci si svolge all'interno del segmento prossimale del tubulo contorto distale e consiste nel blocco del trasportatore $\mathrm{Na}^{+} / \mathrm{Cl}^{-}$con conseguente inibizione del trasporto di $\mathrm{NaCl}$ dal sito luminale delle cellule epiteliali. Questo porta a un aumento nella concentrazione di sodio nel tubulo contorto distale e nel dotto collettore. Tuttavia l'aumento del flusso del $\mathrm{Na}+$ nel dotto collettore provoca l'attivazione di meccanismi aldosterone mediati, che portano ad un aumentato riassorbimento del $\mathrm{Na}+$ nei segmenti più distali del nefrone e contestuale escrezione degli ioni $\mathrm{H}+$ e $\mathrm{K}+$ nelle urine.

Un altro effetto dei tiazidici è l'aumento del riassorbimento del calcio a livello del tubulo contorto prossimale (secondario al depauperamento del volume indotto dal diuretico che incrementa il riassorbimento di $\mathrm{Na}^{+}$e passivo di $\mathrm{Ca}_{2}^{+}$) e distale (mediante l'attività del trasportatore $\mathrm{Na}^{+} / \mathrm{Ca}_{2}{ }^{+}$nella membrana baso-laterale, incrementata dalla riduzione del $\mathrm{Na}^{+}$intracellulare per effetto delle tiazidici).

\section{Diuretici risparmiatori di potassio}

Riducono il riassorbimento del $\mathrm{Na}^{+}$nei tubuli collettori e nei dotti. In questa sede il riassorbimento del $\mathrm{Na}^{+}$e l'escrezione di $\mathrm{K}^{+}$sono regolati dall'aldosterone e questa classe di farmaci agisce proprio a tale livello. È probabile che sia abbiano effetti simili anche su quello di $\mathrm{H}^{+}$da parte delle cellule intercalate del dotto collettore, che in parte spiegano l'acidosi metabolica osservata con gli antagonisti aldosteronici.

Lo spironolattone e l'eprelenone si legano direttamente al recettore dell'aldosterone, riducendo anche la formazione intracellulare di metaboliti attivi. Amiloride e triamterene non bloccano tale recettore, ma interferiscono direttamente con l'ingresso del $\mathrm{Na}+$ attraverso i canali ionici per il sodio nella membrana apicale del dotto collettore. Siccome la secrezione di $\mathrm{K}^{+}$a questo livello è accoppiata con l'ingresso di $\mathrm{Na}^{+}$, queste sostanze sono anche efficaci diuretici risparmiatori di $\mathrm{K}^{+}$.

\section{Vaptani}

Agiscono inibendo l'attività dell'orome antidiuretico (ADH) sul dotto collettore dove avviene la concentrazione finale delle urine. In tale sito, infatti, l'ADH controlla la permeabilità di questo segmento all'acqua regolando l'inserimento di canali per l'acqua preformati (acquaporina-2) nella membrana apicale attraverso un meccanismo mediato da cAMP accoppiato a proteina $\mathrm{G}$.

\section{Inibitori del co-trasportatore del glucosio sodio-dipendente 2}

Rappresentano una nuova classe di farmaci inizialmente indicati nel trattamento del diabete mellito, che attualmente trovano un possibile utilizzo anche nel trattamento dello scompenso cardiaco. I recettori del glucosio sodio-dipendente 2 (SGLT2) sono espressi nella superficie luminale del segmento $\mathrm{S} 1$ del tubulo contorto prossimale e nelle cellule alfa pancreatiche e sono responsabili del riassorbimento del $90 \%$ del glucosio filtrato dal glomerulo. Il traporto attivo del glucosio attraverso questi recettori è connesso al trasporto del $\mathrm{Na}+$ che viene mantenuto dalla sua escrezione attiva nell'interstizio grazie alla pompa $\mathrm{Na}+\mathrm{K}+$ ATPasi presente sulla membrana basolaterale delle sopradette cellule renali. La concentrazione plasmatica oltre il quale avviene l'escrezione urinaria di glucosio è di circa 180$200 \mathrm{mg} / \mathrm{dL}$, ma nel diabetico questo livello si innalza a $300 \mathrm{mg} / \mathrm{dL}$ per l'aumento dell'attività dell'SGLT2.

L'attività di questa nuova classe di farmaci sposta tale soglia a $50 \mathrm{mg} / \mathrm{dL}$, determinando una riduzione del $30-50 \%$ del riassorbimento del glucosio filtrato. L'effetto finale sarà un aumento della glicosuria, una riduzione del glucosio plasmatico e dei valori di emoglobina glicata, indipendentemente dall'insulina e siccome la glicosuria si verifica solo in presenza di iperglicemia, il rischio di ipoglicemia connesso a questi farmaci è basso. Addizionalmente, dato che il $\mathrm{Na}^{+}$ è co-trasportato con il glucosio, si verifica un aumento della diuresi osmotica e della natriuresi, con una secondaria riduzione del volume plasmatico e della pressione arteriosa, del precarico ventricolare, della tensione di parete e delle pressioni di riempimento. Inoltre, la riduzione della stiffness parietale arteriosa e delle resistenze vascolari periferiche e altri effetti cardioreno protettivi sono stati riconosciuti agli inibitori del SGLT2.

\section{La resistenza ai diuretici ed il blocco del nefrone}

La resistenza ai diuretici rappresenta un fattore prognostico negativo nei pazienti affetti da scompenso cardiaco. Seppure esistano molteplici definizioni, la si può generalmente identificare come l'impossibilità a risolvere lo stato congestizio nonostante l'utilizzo di appropriate dosi di diuretici. A causa della mancanza di una definizione universalmente riconosciuta, è difficile quantificarne esattamente la prevalenza. Alcuni recenti studi, stimano che la prevalenza di tale condizioni si attesti attorno al $20-30 \%$ tra i pazienti con insufficienza cardiaca. ${ }^{9,10}$

I meccanismi che portano ad una ridotta risposta 
ai diuretici nello scompenso cardiaco sono molteplici: in primo luogo, dopo un periodo di natriuresi, la concentrazione plasmatica e quindi tubulare del diuretico tende a ridursi, il riassorbimento tubulare di sodio non viene più inibito e subentra un periodo di anti-natriuresi; in secondo luogo con l'avanzare dello scompenso cardiaco si verifica una perdita della responsività ai peptidi natriuretici endogeni. Infine i diuretici aumentano il riassorbimento di soluti nei segmenti distali del nefrone, determinando ipertrofia ed iperplasia delle cellule epiteliali. ${ }^{11,12}$

La mancata aderenza alle indicazioni comportamentali e terapeutiche, nonché l'assunzione di farmaci che influenzano negativamente la funzione renale (come i FANS) devono sempre essere indagate nell'ambito di una presunta resistenza ai diuretici.

La somministrazione contemporanea di due classi differenti di diuretici è una scelta terapeutica comune ed efficace quando la somministrazione del solo diuretico dell'ansa non consente di ottenere la riduzione desiderata del volume di liquidi extracellulare. L'aggiunta di un diuretico del tubulo prossimale o del tubulo collettore distale è spesso eccezionalmente efficace; quando si aggiunge un secondo diuretico, in generale, non dovrebbe essere modificata la dose del diuretico dell'ansa, perché l'andamento della curva dose-risposta di quest'ultimo non è influenzata dall'aggiunta di altri diuretici. La combinazione di un diuretico dell'ansa e del tubulo collettore distale è efficace grazie a diversi meccanismi; in primo luogo $\mathrm{i}$ diuretici tiazidici hanno emivite lunghe e possono pertanto attenuare la ritenzione post-diuretica di $\mathrm{NaCl}$; inoltre l'inibizione del riassorbimento di sodio nel tubulo distale è fondamentale per evitare l'aumentato riassorbimento di soluti delle cellule epiteliali distali.

Questo approccio consente di bloccare in modo sequenziale la parte tubulare del nefrone.

La scelta del diuretico tiazidico da usare come secondo diuretico è soggettiva; in molti casi il metolazone è il farmaco preferito per la sua lunga emivita e perché ha mostrato una buona efficacia anche in caso di bassa velocità di filtrazione glomerulare. I diuretici del tubulo distale possono essere aggiunti al dosaggio massimo (es. metolazione 2,5-10 mg o idroclorotiazide 50-100 $\mathrm{mg}$ ). Tale approccio terapeutico tuttavia causa in molti casi una eccessiva deplezione di liquidi ed elettroliti per cui è necessario uno stretto monitoraggio clinico e laboratoristico; generalmente quando si è ottenuto un discreto controllo del sovraccarico idrico è opportuna la rapida riduzione del diuretico tiazidico, che può essere somministrato ad esempio a giorni alterni. ${ }^{13}$

Nella maggior parte dei casi l'aggiunta di un risparmiatore di potassio è fondamentale per evitare lo sviluppo di ipokaliemia, potenziando ulteriormente l'effetto diuretico.

Nelle fasi avanzate dello scompenso cardiaco un fattore realmente limitante la risposta alla terapia diuretica è lo sviluppo della sindrome cardio-renale. Il peggioramento degli indici di funzionalità renale nell'ambito delle cardiopatie è solitamente considerato ad eziologia pre-renale, anche se in molti casi la sola componente emodinamica non giustifica pienamente lo sviluppo dell'insufficienza renale. In realtà i meccanismi alla base della sindrome cardio-renale ed il suo trattamento sono ancora poco conosciuti. ${ }^{14}$

\section{Conclusioni}

La congestione è il sintomo più frequente dell'insufficienza cardiaca. La sua gestione, soprattutto nelle fasi avanzate dello scompenso, può essere complessa. La conoscenza della farmacodinamica dei farmaci diuretici è fondamentale per il loro corretto utilizzo ed associazione, al fine di ottenere un blocco sequenziale del nefrone e massimizzare l'effetto diuretico globale.

\section{Bibliografia}

1. Chioncel, O. et al. Clinical phenotypes and outcome of patients hospitalized for acute heart failure: the ESC Heart Failure Long- Term Registry. Eur. J. Heart Fail. 19, 1242-1254 (2017).

2. Martens, P., Nijst, P. \& Mullens, W. Current approach to decongestive therapy in acute heart failure. Curr. Heart Fail. Rep. 12, 367-378 (2015).

3. Fallick, C., Sobotka, P. A. \& Dunlap, M. E. Sympathetically mediated changes in capacitance: redistribution of the venous reservoir as a cause of decompensation. Circ. Heart Fail. 4, 669-675 (2011)

4. Nijst, P. et al. The pathophysiological role of interstitial sodium in heart failure. J. Am. Coll. Cardiol. 65, 378388 (2015).

5. Wolff, J. J., Laremore, T. N., Busch, A. M., Linhardt, R. J. \& Amster, I. J. Influence of charge state and sodium cationization on the electron detachment dissociation and infrared multiphoton dissociation of glycosaminoglycan oligosaccharides. J. Am. Soc. Mass. Spectrom. 19, 790-798 (2008).

6. Yu, A. S. L. et al. in Brenner and Rector's The Kidney 1708-1740 (Elsevier, 2020).

7. Faris RF, Flather M, Purcell H, Poole-Wilson PA, Coats AJ. Diuretics for heart failure. Cochrane Database Syst Rev 2012;2:CD003838.

8. Ponikowski P, Voors AA, Anker SD, Bueno H, Cleland JG, Coats AJ, Falk V, Gonzalez-Juanatey JR, Harjola VP, Jankowska EA, Jessup M, Linde C, Nihoyannopoulos P, Parissis JT, Pieske B, Riley JP, Rosano GM, Ruilope LM, Ruschitzka F, Rutten FH, van der Meer P. 2016 ESC Guidelines for the diagnosis and treatment of acute and chronic heart failure: the Task Force for the diagnosis and treatment of acute and chronic heart failure of the European Society of Cardiology (ESC). Developed with the special contribution of the Heart Failure Association (HFA) of the ESC. Eur J Heart Fail 2016;18:891-975. 
9. Jentzer JC, Dewald TA, Hernandez AF. Combination of loop diuretics with thiazide-type diuretics in heart failure. JACC. 2010;56:1527---34.

10. Valente MA, Voors AA, Damman K, et al. Diuretic response in acute heart failure: clinical characteristics and prognostic significance. Eur Heart J. 2014;35:1284---93.

11. Diuretic Resistance. Hoorn EJ, Ellison DH. Am J Kidney Dis. 2017 Jan;69(1):136-142.
12. Diuretic Treatment in Heart Failure. Ellison DH, Felker GM. N Engl J Med. 2017 Nov 16;377(20):1964-1975

13. Torrent or torment from the tubules? Challenge of the cardiorenal connections. Stevenson LW, Nohria A, Mielniczuk L. J Am Coll Cardiol. 2005 Jun 21;45(12):2004-7

14. Cardiorenal Syndrome Revisited. Zannad F, Rossignol P. Circulation. 2018 Aug 28;138(9):929-944. 


\title{
Scompenso cardliaco: differenze di genere?
}

\author{
Rosalba Falzone, ${ }^{1}$ Lorenza Lenzi, ${ }^{1}$ Cecilia Politi ${ }^{2}$ \\ ${ }^{1}$ U.O. Medicina Interna Rovereto, APSS TN, Trento; ${ }^{2}$ U.O.C. Medicina Interna Isernia - ASREM, Isernia, Italia
}

\section{Introduzione}

Dobbiamo sfatare una serie di miti che considerano erroneamente l'infarto e l'ictus patologie che colpiscono prevalentemente l'uomo, mentre la donna teme principalmente di morire di tumore ed in particolare di tumore della mammella. Ogni donna ha una probabilità su due di morire per malattia vascolare (ictus, infarto, scompenso cardiaco...) mentre solo una su diciassette morirà per tumore della mammella. Nel 2014 in Europa muore di patologia cardiovascolare il $42 \%$ degli uomini e ben il 51\% delle donne (Figura 1). I dati ISTISAN 2016 confermano anche in Italia (Figura 2) la maggiore mortalità vascolare per ictus, infarto e scompenso cardiaco (SC) della donna (38.7\%) rispetto all'uomo (48.4\%).

Lo SC, che è oggi una vera e propria epidemia in crescita, ha caratteristiche diverse nella donna ${ }^{1} \mathrm{e}$ dopo i 75 anni colpisce più donne che uomini (Figura 3 ). ${ }^{2}$ Lo scompenso cardiaco nella donna è correlato maggiormente all'ipertensione, alla obesità ed è frequentemente complicato dalla fibrillazione atriale. Lo SC è però una patologia esclusivamente femminile in tre condizioni: la cardiomiopatia peripartum (associato alla gravidanza), la cardiomiopatia da stress e dopo i trattamenti per neoplasia della mammella.

Un altro aspetto deve far riflettere: malgrado una documentata maggiore mortalità per malattia vascolare, i farmaci antipertensivi, anticoagulanti, antitrombotici, antidiabetici, ecc., vengono meno prescritti nella donna, ${ }^{3}$ così come le procedure diagnostiche $\mathrm{e}$ terapeutiche invasive. ${ }^{4}$

Le donne inoltre, specie le più anziane e quelle di

Corrispondente: Cecilia Politi, U.O.C. Medicina Interna Isernia - ASREM, Isernia, Italia.

E-mail: cecilia.politi@asrem.org

Articolo pubblicato secondo la Creative Commons Attribution NonCommercial 4.0 License (CC BY-NC 4.0).

${ }^{\circ}$ Copyright: the Author(s), 2021

Licensee PAGEPress, Italy

QUADERNI - Italian Journal of Medicine 2021; 9(2):e3 razza nera, meno facilmente raggiungono gli obiettivi pressori ed i valori glicemici consigliati dalle linee guida. ${ }^{5}$ È quindi fondamentale che aumenti sia nei medici che nella popolazione generale, la conoscenza delle differenze di genere nelle malattie vascolari.

\section{Differenze di genere}

\section{Dati epidemiologici}

Lo scompenso cardiaco, che in numeri assoluti colpisce più le donne che gli uomini, ${ }^{6}$ è responsabile del 35\% della mortalità cardiovascolare.

La prevalenza dello scompenso cardiaco tra i 45 ed i 75 anni è compresa tra 1,6 e 4,6 casi per 1000 nel maschio e tra 0,9 e 2,2 casi nella femmina; circa $1{ }^{\prime} 1 \%$ dei maschi svilupperà uno scompenso cardiaco dopo i 75 anni e ben il $2 \%$ delle femmine dopo gli 80 anni.

Il $40 \%$ dei pazienti muore entro un anno dalla prima ospedalizzazione e la sopravvivenza ad oltre 5 anni dalla diagnosi è del $25 \%$ nel maschio e del $38 \%$ nella femmina. Questo dato e la maggiore longevità femminile giustificano il maggior tasso di ospedalizzazione e di dimissione nelle donne $>80 \mathrm{anni}^{7}$ con una previsione di costi stimati, che nel 2030 raggiungerà i quasi 70 bilioni di dollari negli USA. ${ }^{8}$

Anche In Italia, i tassi di ospedalizzazione del Ministero della salute per SC vedono circa 200.000 ricoveri/anno con una lieve prevalenza femminile ( 99.033 $\mathrm{M} v s$ 105.840 F) nel rapporto ISTISAN 10/43 del 2010.

Ricordando che lo scompenso cardiaco è la prima causa di ricovero ospedaliero dopo il parto ed è il primo DRG della Medicina Interna, le caratteristiche dei pazienti con SC variano a seconda dei reparti di ricovero. Se in Cardiologia secondo il registro AMCO IN-CHF un 50\% dei pazienti ha meno di 70 anni, con un rapporto maschi/femmine di 7/10, in Medicina Interna osserviamo invece una età media più elevata (76,6 anni $\mathrm{M}$ vs 80,7 anni F) e con una lieve prevalenza numerica femminile ( $48 \% \mathrm{M} v s 52 \% \mathrm{~F}) .{ }^{9}$

\section{Meccanismi fisiopatologici}

Le differenze nello scompenso cardiaco tra uomo e donna potrebbero essere riferibili ad una serie di elementi (Tabella 1). ${ }^{6,10-14}$ 


\section{Caratteristiche cliniche}

La donna con scompenso cardiaco presenta più frequentemente all'ecocardiogramma rispetto all'uomo una frazione di eiezione conservata ( $\mathrm{pEF}$ $\geq 50 \%$ ) o mid-range (mEF $40-49 \%) .{ }^{15}$ La prevalenza dello scompenso a $\mathrm{pEF}$ è quasi il doppio nella donna rispetto all'uomo. Nello scompenso di nuova insor- genza inoltre, le donne hanno un $65 \%$ in meno di probabilità di avere il riscontro di una $\mathrm{EF}$ ridotta $(\mathrm{rEF})$ all'ecocardiogramma, dati confermati sia dal Framingham che dall'Olmstead County database. ${ }^{16,17}$

Il quadro clinico e la mortalità dello scompenso cardiaco sia a rEF che $\mathrm{pEF}$, sostanzialmente non differiscono. ${ }^{18}$

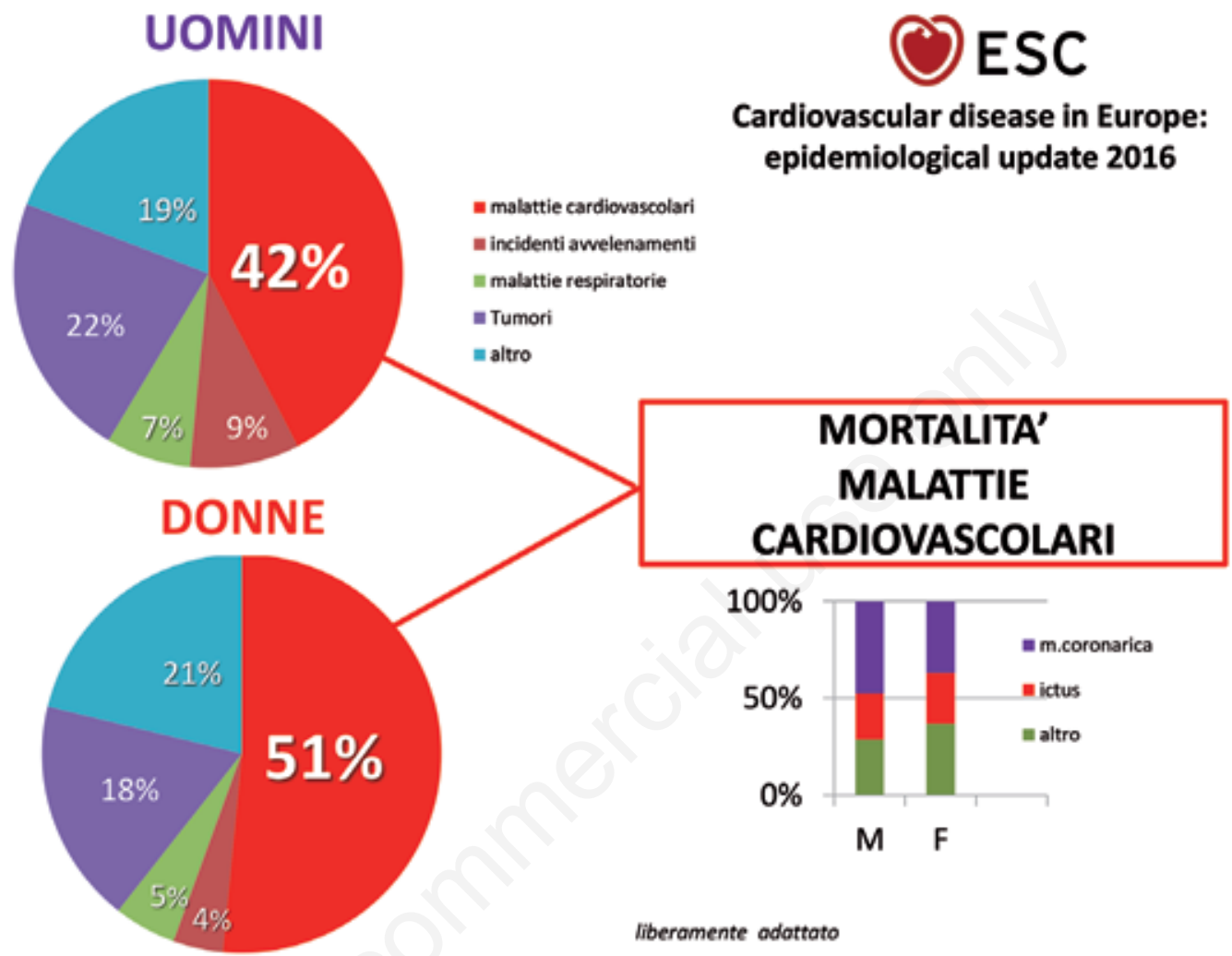

Figura 1. Malattie cardiovascolari in Europa - dati epidemiologici del 2014.

Uomini

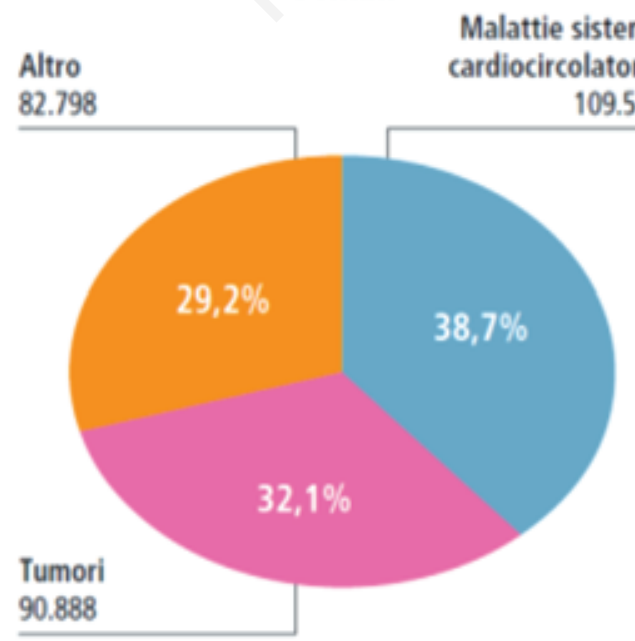

Donne

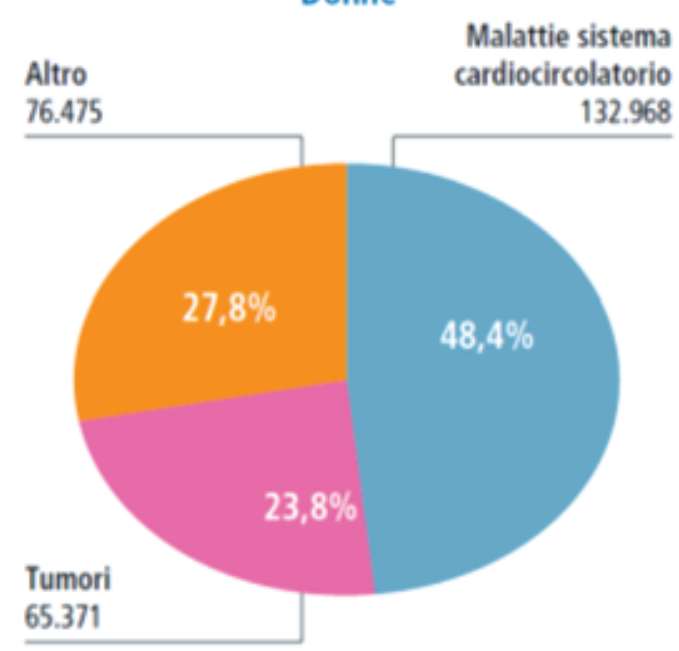

Figura 2. Mortalità in Italia - Dati ISTISAN 2016. 
La coesistenza in una donna di SC a pEF, obesità e fibrillazione atriale (che più frequentemente complica lo scompenso cardiaco rispetto all'uomo) costituisce inoltre quello che gli anglosassoni definiscono il terzetto malefico,${ }^{19}$ sottolineando la fragilità di questa specifica popolazione. La fibrillazione atriale (FA) è infatti un riconosciuto fattore di rischio per ictus, con un rischio quasi doppio nella donna, ma la FA è fattore di rischio anche per lo scompenso cardiaco, indipendentemente dal tipo di FA. ${ }^{20}$

Il rapporto stretto tra obesità (body mass index $>30$ $\mathrm{kg} / \mathrm{m}^{2}$ ) e l'aumento della incidenza di scompenso cardiaco è stato infatti dimostrato chiaramente in una metanalisi di studi condotti su oltre 600.000 partecipanti. $^{21}$

\section{Fattori di rischio e prevenzione}

Lo scompenso cardiaco è una patologia prevenibile in entrambi i sessi. In post-menopausa anche in assenza di malattia coronarica, ipertensione e diabete mellito, l'adozione di corretti stili di vita riduce il rischio di scompenso, sottolineando l'importanza della prevenzione sia primaria che secondaria, anche nella donna. ${ }^{22}$

Grande attenzione va quindi posta ai fattori di rischio cardiovascolari modificabili e non modificabili; le donne non solo hanno un rischio vascolare maggiore rispetto agli uomini per un peso maggiore dei fattori di rischio tradizionali ma, presentano anche dei fattori di rischio non tradizionali o emergenti (nascita pretermine, ipertensione in gravidanza, diabete gestazionale, malattie autoimmuni, trattamenti per neoplasia della mammella, depressione) che ne condizionano lo stato di salute o di malattia, se non adeguatamente riconosciute, trattate e seguite nel tempo.

\section{Terapia}

Le Linee guida della Società Europea di Cardiologia (ESC) del 2016, nel rimarcare che devono essere garantiti la diagnosi e la terapia più adeguata a uomini

Tabella 1. Scompenso cardiaco: differenze uomo/donna.

Diversa etiologia. Lo scompenso cardiaco della donna anziana con frazione di eiezione preservata si correla prevalentemente alla presenza di ipertensione arteriosa, diabete e alla cardiopatia valvolare; mentre nell'uomo lo scompenso cardiaco a frazione di eiezione ridotta, è correlato maggiormente alla cardiopatia ischemica ${ }^{6,10}$

Effetti cardioprotettivi degli estrogeni sul rimodellamento cardiaco per la aumentata produzione dei peptidi natriuretici atriali e la ridotta espressione dell'ACE e del TNF alfa dei miociti. ${ }^{11}$ L'infiammazione e la conseguente fibrosi sono ridotte nella donna in pre menopausa grazie all'azione antinfiammatoria degli estrogeni, con un ruolo cardio protettivo sull'endotelio; effetto che scompare dopo la menopausa. Il testosterone ha invece effetti negativi nel remodelling del cuore maschile ${ }^{12}$

Differenti risposte adattive all'invecchiamento e allo stress con aumento della fibrosi negli uomini maggiore rispetto alle donne ${ }^{13}$

Maggiore adattamento ipertrofico concentrico nella donna, ed ipertrofico eccentrico/dilatativo nell' uomo, ${ }^{10}$ le donne con scompenso cardiaco a frazione di eiezione conservato hanno cuori di minori dimensioni e più rigidi

Minor incidenza di morte improvvisa in tutte le fasce di età (per età $<75$ anni: nei maschi $1 / 9 \mathrm{M} v s$ nelle donne $1 / 30$ ), ${ }^{14}$ forse per il minore carico fibrotico

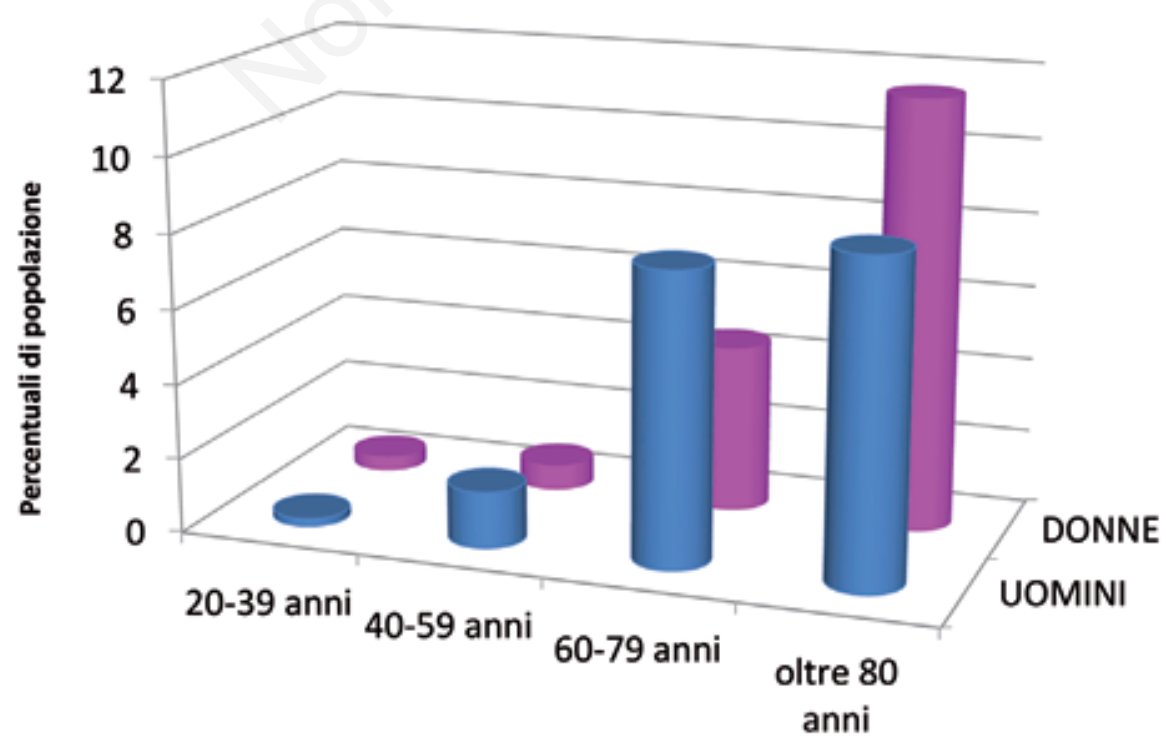

UOMINI

DONNE

Figura 3. Prevalenza di scompenso cardiaco per sesso ed età. 
e donne con scompenso cardiaco, nelle raccomandazioni sulla gestione dello SC a pEF e mEF, sottolineano l'importanza della valutazione e del trattamento delle principali e più frequenti comorbilità sia cardiovascolari che non. Sono infatti indicate specifiche raccomandazioni (evidenza grado IC) sul trattamento di: ipertensione arteriosa, cardiopatia ischemica, dislipidemie, diabete mellito, apnee notturne e artrite per migliorare la sintomatologia, il benessere del paziente e la prognosi, prevenendo o ritardando lo scompenso manifesto e la morte. La Società Europea di Cardiologia nel position paper del 2019, non indica differenze, tra uomini e donne, nel trattamento del diabete nel paziente con scompenso. ${ }^{23}$

Nella pratica clinica e nei trial cinici, i pazienti con $\mathrm{SC}$ a $\mathrm{pEF}$ e $\mathrm{mEF}$, ricevono in misura minore i farmaci specifici per il trattamento dello scompenso cardiaco: diuretici, $\beta$-bloccanti (BB), antialdosteronici (MRAs) e ACE inibitori (ACEIs) o antagonisti dell'angiotensina II (ARBs). ${ }^{24}$

Nello scompenso cardiaco con pEF rispetto allo scompenso con rEF non vi sono evidenze dirette che supportino l'uso di un trattamento farmacologico specifico. Fondamentale è quindi la gestione della sintomatologia e delle comorbilità.

Sulla riduzione della mortalità nello $\mathrm{SC}$ a pEF, i trial con ACEIs, ARBs BB e MRAs hanno fallito l'obiettivo.

Per quanto riguarda l'utilizzo degli inibitori del sistema angiotensina-neprilisina (ARNI), lo studio PARAGON che ha dimostrato l'efficacia del del sacubitril-valsartan nello $\mathrm{SC}$ a rEF, non ha raggiunto nei pazienti con $\mathrm{SC}$ a pEF l'end point previsto, anche se è stata dimostrato un beneficio in alcuni sottogruppi, tra cui le donne. ${ }^{25}$

Differente tra uomini e donne è anche la risposta alla terapia di risincronizzazione (CRT). Questa opzione terapeutica è molto importante nei pazienti con $\mathrm{rEF}$ $(<35 \%)$ in terapia farmacologica ottimale ed in III-IV classe funzionale NHYA. Le donne sottoposte a CRT rispetto agli uomini, presentano una maggiore riduzione del rischio di mortalità per tutte le cause, morte o ospedalizzazione per scompenso e per aritmie ventricolari, con un rimodellamento cardiaco, ecograficamente dimostrato, più evidente. ${ }^{26}$ Eppure le donne sono sottorappresentate nei trial clinici sulla CRT $(<30 \%)$ e questa procedura viene loro offerta in misura inferiore e nelle fasi più avanzate di malattia. Questa opportunità terapeutica deve essere offerta a tutti coloro che ne hanno la indicazione, superando il gap di genere. ${ }^{26,27}$

Se le linee guida non differiscono nelle indicazioni al trattamento dello scompenso cardiaco nell'uomo e nella donna, ${ }^{28}$ va però sottolineato che il numero di donne arruolate nei principali studi sui farmaci utilizzati nello scompenso è stato quasi sempre al disotto del $30 \%$, non raggiungendo la significatività statistica. ${ }^{29}$

L'utilizzo nella donna di dosi ridotte di $\beta$-bloc- canti, ARBs e ACEIs per una migliore sopravvivenza, ${ }^{30}$ necessita di studi appropriati con dati stratificati by gender.

Infine le donne sono meno sottoposte a trapianto cardiaco, e le riceventi sono più giovani rispetto agli uomini. Le donne inoltre sono più frequentemente donatrici, a conferma dell'esistenza di un bias di genere. ${ }^{31}$

\section{Cardiomiopatia peripartum}

Il gruppo di lavoro della Heart Failure Association (HFA) dell'ESC ha definito la cardiomiopatia peripartum (PPCM) nella messa a punto del 2016, come scompenso cardiaco acuto secondario a disfunzione sistolica sinistra $(L V E F<45 \%)$ che si presenta frequentemente alla fine della gravidanza o nel mese successivo al parto, senza precedenti cause di scompenso. ${ }^{32}$

Il meccanismo fisiopatologico prevede un modello a due tempi di uno squilibrio angiogenico cardiaco durante il periodo del peripartum, combinando segnali antiangiogenici sistemici durante le fasi avanzate della gravidanza e la suscettibilità dell'ospite, attraverso una insufficiente difesa proangiogenica localizzata nel cuore. ${ }^{33,-36}$ Questo squilibrio può essere ulteriormente innescato dallo stress ossidativo per l'attivazione della catepsina $\mathrm{D}$, una proteasi responsabile della scissione della prolattina nell' angiostatico e proapoptotico subframmento $16 \mathrm{kDa}^{37}$

I tassi di mortalità della PPMC variano dal $<5 \%$ al $50 \%$; la tachiaritmia ventricolare ne è spesso la causa, e principalmente durante i primi 6 mesi. ${ }^{38}$

L'alta mortalità della PPCM rappresenta una sfida, in quanto l'intervento terapeutico deve considerare la salute sia della madre che del prodotto del concepimento.

La sintomatologia della PPCM è quella tipica dello scompenso cardiaco, alcuni sintomi possono mimare le alterazioni fisiologiche durante o dopo la gravidanza, e potrebbero ritardare la diagnosi. Nella PPMC acuta, la diagnosi differenziale include: miocardite, preesistente cardiomiopatia, malattia valvolare o cardiaca congenita. In caso di shock cardiogeno vanno immediatamente esclusi l'infarto del miocardio associato alla gravidanza, l'embolia polmonare e l'embolismo da liquido amniotico.

La diagnosi di PPMC che è una diagnosi di esclusione, prevede l'esecuzione di un ECG che è raramente normale (l'assenza di anomalie della ripolarizzazione ha un alto valore predittivo negativo $^{39}$ ), dei peptidi natriuretici (per identificare la causa cardiaca della dispnea) e l'ecocardiografia (per la conferma diagnostica e/o l'esclusione di copatologie e/o complicanze). La risonanza magnetica nucleare $(\mathrm{RMN})$ non è necessaria di routine e la somministrazione di gadolinio va ritardata fino al parto, a meno di una necessità assoluta. La biopsia endomiocardica non aggiunge informazioni diagnostiche o prognostiche in 
caso di PPCM ma, se necessaria per escludere una miocardite acuta, va procrastinata a dopo il parto. ${ }^{40}$

Il management dello scompenso acuto provocato dalla PPCM, nelle fasi iniziali non differisce dal trattamento dello scompenso da etiologie differenti ${ }^{41-43} \mathrm{e}$ presenta specifiche peculiarità che richiedono un approccio multidisciplinare (Tabella 2).

\section{Cardiomiopatia da stress (Takotsubo)}

La cardiomiopatia di Takotsubo (TTS) è una sindrome caratterizzata da transitoria disfunzione regionale acuta del ventricolo sinistro, in assenza di una significativa malattia coronarica. Si riscontra prevalentemente nelle donne (F/M: 9/1) con età media di 66 anni. ${ }^{44}$

La precisa fisiopatologia della TTS non è ancora del tutto compresa. Studi recenti hanno dimostrato che alterazioni specifiche della risposta neurologica e dell'attivazione simpatica in seguito ad uno stimolo emozionale (morte di un familiare, abuso domestico, discussioni, diagnosi di malattia grave, devastanti perdite finanziarie o di gioco, catastrofi naturali) o fisico (insufficienza respiratoria acuta, complicanze decorso post-chirurgico, fratture, infezioni, malattie neurologiche) rivestono un ruolo centrale, confermando l'importanza dell'interazione cuore-cervello. ${ }^{45,46}$
Sono stati proposti dei nuovi criteri diagnostici, riassunti in otto punti nella Tabella $3 .{ }^{47}$

Dopo la coronarografia, qualora le alterazioni cinetiche parietali siano limitate alla distribuzione di un singolo vaso, la diagnosi differenziale con una sindrome coronarica acuta (SCA) o con la miocardite richiederà la esecuzione di una risonanza magnetica cardiaca. ${ }^{48} \mathrm{Le}$ caratteristiche cliniche e la prognosi della TTS possono variare ampiamente. Una nuova classificazione del rischio basata sul tipo di trigger (la classificazione InterTAK) ha rilevato nella donna anziana, che l'evento secondario a malattia neurologica così come quello associato a fattori di stress fisico, condizioni mediche o procedure, ha una prognosi peggiore a breve termine ed una mortalità a lungo termine più elevata, rispetto $a$ quello associato a fattori scatenanti emotivi. ${ }^{49}$

Una bassa frazione di eiezione del ventricolo sinistro (LVEF) al momento del ricovero, ha dimostrato essere un predittore indipendente di mortalità intraospedaliera e a lungo termine. ${ }^{50}$

Anche la fibrillazione atriale è stata identificata come un predittore di prognosi infausta a breve e lungo termine, probabilmente a causa delle conseguenze emodinamiche della perdita della contrazione atriale e dell'aumentato rischio di eventi tromboembolici. ${ }^{51}$

Tabella 2. Suggerimenti per la gestione della cardiomiopatia peripartum.

Evitare durante la gravidanza e l'allattamento i farmaci per lo SC a tossicità fetale (ACEi/ARBs, MCRA), successivamente introdurre lo standard terapeutico dello SC

Ipotizzare in via sperimentale l'uso della bromocriptina (2,5 mg BID per 2 settimane seguita da 2,5 mg/die per 6 settimane)

Anticoagulare con eparina per la prevenzione delle complicanze cardio-emboliche se $\mathrm{FE} \leq 35 \%$ o trattamento con bromocriptina

Considerare come inotropo di prima linea il levosimendan $(0,1 \mu \mathrm{g} / \mathrm{kg} / \mathrm{min}$ per $24 \mathrm{~h})$ invece delle catecolamine in caso di shock cardiogeno

Considerare precocemente l'impianto del defibrillatore per la prevenzione della morte improvvisa ${ }^{43}$ se $\mathrm{FE} \leq 35 \%$

Contemplati il supporto meccanico circolatorio: contropulsatore aortico, ECMO (extracorporeal life support), device di assistenza al ventricolo sx

Tabella 3. Criteri diagnostici cardiomiopatia di Takotsubo.

1. Disfunzione acuta transitoria del ventricolo sinistro (VS) (ipocinesia, acinesia o discinesia) che può coinvolgere tipicamente la regione apicale ma anche le pareti medio-ventricolari e basali o causare alterazioni segmentarie, può essere interessato anche il ventricolare destro. Le anomalie del movimento della parete regionale che si estendono oltre una singola distribuzione vascolare epicardica sono considerate una caratteristica importante che distingue la TTS dall'infarto miocardico classico

2. Un trigger emotivo, fisico o combinato può, ma non obbligatoriamente, precedere l'evento

3. Malattie neurologiche (ictus, emorragia subaracnoidea, attacco ischemico transitorio o convulsioni...) e anche il feocromocitoma possono essere eventi scatenanti

4. Alterazioni ECGrafiche frequenti sono: innalzamento diffuso del segmento ST anteriore o in diverse derivazioni, sottoslivellamento ST, inversione dell'onda T e prolungamento dell'intervallo QTc. Esistono casi rari senza modifiche all'ECG

5. I livelli dei biomarcatori cardiaci (troponina e creatinchinasi) sono moderatamente elevati nella maggior parte dei casi, spesso non proporzionati con la gravità e la diffusione delle anomalie del movimento della parete regionale

6. È comune un aumento significativo del peptide natriuretico di tipo B

7. Una sindrome coronarica acuta (SCA) non deve essere considerata un criterio di esclusione: TTS e cardiopatia ischemica possono coesistere

8. La miocardite infettiva deve essere esclusa ${ }^{47}$ 
Nella fase acuta, i pazienti possono manifestare complicanze meccaniche del ventricolo sinistro (rottura parete libera o del setto), ostruzione del tratto di efflusso del ventricolo sinistro (LVOTO), shock cardiogeno (CS), aritmie ed eventi tromboembolici.

I seguenti fattori sono correlati ad un maggior rischio di evoluzione sfavorevole di TTS: ${ }^{52}$ TTS apicale, stress fisico come causa scatenate, ridotta EF, FA, diabete mellito.

\section{Trattamento}

Non sono disponibili studi clinici randomizzati sul trattamento della TTS, per cui viene consigliato un approccio medico incentrato sul trattamento tempestivo di supporto delle complicanze e sulla personalizzazione della terapia a lungo termine. La gestione acuta della TTS dovrebbe essere basata sulla esclusione della presenza di una SCA, sulla stratificazione del rischio principalmente sulla base della LVEF, e garantendo il monitoraggio elettrocardiografico per almeno 48 ore. Gli esami ecocardiografici seriati sono mandatari per la valutazione del riempimento cardiaco e della funzione ventricolare, nonché per l'identificazione precoce di qualsiasi complicanza meccanica, come LVOTO.$^{53}$ Se la LVEF è del $35-45 \%$, devono essere presi in considerazione i farmaci per l'insufficienza cardiaca, inclusi ACEIs, ARBs e i BB..$^{54}$

Nei pazienti con LVOTO è indicata la prevenzione dell'ipovolemia con un'appropriata infusione di cristalloidi; la sua mancata rilevazione induce all'uso di diuretici, vasodilatatori o agenti inotropi che possono aggravare l'ostruzione e innescare un pericoloso circolo vizioso. Alcuni studi suggeriscono che i BB possono ridurre il LVOTO diminuendo l'ipercontrattilità basale, aumentando il riempimento del ventricolo sinistro e riducendo la frequenza cardiaca. ${ }^{55}$ L'ivabradina può ridurre la frequenza cardiaca senza alterare la contrattilità miocardica e potrebbe essere un'alternativa ai $\mathrm{BB}$, specialmente quando la tachicardia è associata a una funzione miocardica gravemente depressa. ${ }^{56}$

Il ballooning apicale con alti livelli di troponina al momento del ricovero è stato descritto come un marker di alto rischio tromboembolico legato al picco di catecolamine, alla disfunzione endoteliale e alla stasi ematica, causate dall'acinesia della parete regionale. Nonostante l'alto rischio, il ruolo della anticoagulazione orale in profilassi primaria resta ancora da determinare. ${ }^{54} \mathrm{Nel}$ caso in cui venga rilevato un trombo ventricolare, l'anticoagulazione orale viene raccomandata per 1-3 mesi oppure fino alla risoluzione trombo e/o alla ripresa della funzione del VS..$^{54}$

L'uso dell'aspirina da sola o in combinazione con clopidogrel, rappresenta un predittore indipendente di una minore incidenza di eventi cardiaci durante il ricovero, mentre non riduce la recidiva di TTS. ${ }^{57,58}$

Anche l'aggiunta di BB e ACEIs può contribuire a ridurre la mortalità, sebbene non vi siano indicazioni chiare sul trattamento farmacologico a lungo termine. Tutti i pazienti con TTS dovrebbero essere seguiti per valutare il completo recupero ventricolare, gestire i sintomi a lungo termine e rilevare le recidive. Inoltre, le comorbidità dei pazienti devono essere attentamente monitorate perché la maggior parte della mortalità a lungo termine nella TTS sembra correlata a condizioni non cardiache coesistenti. È stato inoltre suggerito il trattamento di eventuali fattori di stress neurologici o psichiatrici, perché riscontrati più frequentemente in caso di recidiva di TTS. ${ }^{59}$

\section{Cardiomiopatia nella donna con neoplasia mammaria}

I punti chiave sono sintetizzati nella Tabella $4 .{ }^{60,61}$

La neoplasia mammaria rappresenta la più frequente neoplasia nelle donne e condivide alcuni fattori di rischio con la patologia cardiovascolare, in particolare l'età, l'obesità, l'abitudine tabagica e perfino alcune mutazioni tipo la presenza di BRCA1. ${ }^{62}$

L'incidenza di tale patologia e la ridotta mortalità, grazie alle strategie di trattamento spesso combinate, hanno determinato un crescente numero di donne lungoviventi, e in questo gruppo la causa di mortalità è diventata la patologia cardiovascolare e non la neoplasia stessa. ${ }^{63}$

Vivere con o oltre il cancro ${ }^{64}$ diventa uno slogan che permette di entrare nella dimensione del problema. Nel 2010 in Italia 2.637.975 pazienti erano in

Tabella 4. Punti chiave scompenso cardiaco e neoplasia mammaria nella donna.

Le donne affette da neoplasia mammaria che vengono trattate con farmaci potenzialmente cardiotossici/radioterapia sono a rischio di sviluppare scompenso cardiaco e necessitano di stretto controllo dei fattori di rischio cardiovascolare

La frazione di eiezione del ventricolo sinistro deve essere monitorata prima, durante e dopo il trattamento: il valore del $50 \%$ rappresenta il limite inferiore in linea con i valori usati nei trials e registri internazionali. Una riduzione maggiore del 10\% rispetto al valore basale necessita di un controllo stretto nel tempo

In caso di una riduzione maggiore del $10 \%$ al di sotto del limite inferiore di normalità, ACEs inibitori (oARBs) in combinazione con i $\beta$ bloccanti sono raccomandati

ACE inibitori (o ARBs) e $\beta$-bloccanti sono raccomandati nelle pazienti con scompenso cardiaco se sintomatiche 
vita dopo una precedente diagnosi di cancro. Le donne erano 1.443.942 (il 4,8\% della popolazione italiana di sesso femminile) e la neoplasia mammaria rappresenta il 41,9\% di tutte le neoplasie $(604,841)$. Considerando un trend di crescita costante del 3\% per anno, una proiezione del 2020 prevede circa 3.600 .000 pazienti, di cui 1.900 .000 donne. Il 2,6\% delle donne italiane (0.8 milioni) nel 2020 sono viventi dopo la diagnosi di cancro al seno. ${ }^{65}$

Le possibili complicanze cardiovascolari che si possono verificare durante le terapie oncologiche e nel follow up, sono oggi classificate in nove gruppi, in relazione alla fisiopatologia e alle manifestazioni cliniche: ${ }^{66}$ i) disfunzione miocardica e scompenso cardiaco; ii) malattia coronarica; iii) malattia valvolare; iv) aritmie (specialmente farmaci che prolungano l'intervallo QTc); v) ipertensione arteriosa; vi) malattia tromboembolica; vii) malattia vascolare periferica e stroke; viii) ipertensione polmonare; ix) complicanze pericardiche.

Per ottimizzare la gestione è necessario valutare con attenzione i farmaci con potenziale cardiotossico, siano essi chemioterapici che anticorpi monoclonali.

La cardiotossicità tardiva da antracicline, che insorge tipicamente a distanza di anni, è l'esempio più noto; il deterioramento della frazione di eiezione avviene in circa il $10-15 \%$ delle pazienti trattate con la dose standard $\left(240 / \mathrm{m}^{2}\right){ }^{67}$

Fattori contribuenti sono la dose cumulativa, gli schemi di somministrazione rapida, il sesso femminile e l'età avanzata (>65 anni). L'effetto cardiotossico delle antracicline porta alla perdita diretta di cardiomiociti, alla riduzione della contrattilità del muscolo cardiaco e al danno della microcircolazione. Inoltre, l'ulteriore effetto sulle cellule progenitrici cardiache e sui fibroblasti rende il muscolo cardiaco suscettibile all'azione di successivi fattori stressanti, quali comorbidità o l'utilizzo di altri farmaci. Un caso tipico riguarda la concomitante o seguente somministrazione di trastuzumab (ERB2-target therapy) che può precipitare lo scompenso cardiaco. ${ }^{68}$

Nel trattamento della neoplasia mammaria va considerato l'uso della terapia endocrina e della soppressione ovarica che possono portare a modifiche ormonali e metaboliche che contribuiscono allo sviluppo della malattia cardiovascolare. ${ }^{69}$

Nel trattamento del tumore mammario ruolo determinante gioca la radioterapia.

Fin dai primi studi sugli effetti a distanza della radioterapia si è evidenziato un aumentata frequenza delle malattie cardiovascolari e della mortalità correlata. ${ }^{70}$

Gli effetti della radioterapia si possono manifestare come cardiopatia ischemica, cardiomiopatia, patologia del pericardio, disfunzione valvolare e anomalie di conduzione.

Il miglioramento delle tecniche utilizzate in ambito radioterapico ha permesso di ridurre la dose di irradia- zione del cuore. Nonostante ciò, non è chiaro se esista una dose sicura senza rischio, in quanto anche una piccola dose di radiazione può innescare la cascata infiammatoria ed aumentare il rischio di patologia cardiovascolare. ${ }^{71}$

I fattori chiave che influiscono sulla comparsa di cardiotossicità secondaria a radioterapia si possono riassumere: i) campo di irradiazione e dose: complicanze cardiovascolari paiono più frequenti nelle neoplasie localizzate a sinistra; ii) associazione a chemioterapia cardiotossica (antracicline/trastuzumab); iii) la preesistenza dei noti fattori di rischio cardiovascolare (ipertensione, fumo, cardiopatia preesistente) può aumentare il rischio dopo trattamento radioterapico; iv) tempo del follow up: aumento del rischio nella prima decade e aumento della mortalità nella seconda decade. ${ }^{72}$

\section{Riconoscimento e monitoraggio della tossicità cardiaca}

Necessari sono ECG ed ecocardiogramma con la valutazione della LVEF. Quest'ultima metodica è gravata di alcuni bias tra cui la determinazione operatoredipendente e la qualità dell'immagine. Spesso tale esame definisce già la presenza di un danno non permettendo una diagnosi precoce. La riduzione della frazione di eiezione è espressione solo di una delle molteplici tossicità cardiache. Una metodica promettente è l'ecocardiografia strain basata sull'utilizzo del Doppler tissutale o metodiche 3D che studiano il miocardial strain o global longitudinal strain che è una misura della deformazione miocardica.

Ad oggi l'uso dei due biomarcatori troponina e NT PROB NP viene suggerito, in particolare la troponina ha mostrato un alto valore predittivo negativo e pertanto aiuta a riconoscere i pazienti che necessitano di uno stretto controllo cardiologico. ${ }^{73,74}$

\section{Management}

Riguardo alla gestione farmacologica, alcune misure devono essere tenute in considerazione nella pianificazione terapeutica: ridurre la dose cumulativa di antracicline, impiego di schemi infusionali prolungati, impiego di formulazioni liposomiali di antracicline, impiego di cardioprotettori. Rispetto a quest'ultima categoria, non è chiaro se la prevenzione primaria possa essere rilevante solo in pazienti ad alto rischio al baseline, o anche in pazienti a rischio standard che effettuano chemioterapie altamente cardiotossiche. ${ }^{75}$

Il management si basa principalmente su: i) misure comportamentali (correzione degli stili di vita, in particolare dieta, fumo ed esercizio fisico); ii) presidi farmacologici una volta documentato il danno (uso di ACEs inibitori, $\beta$-bloccanti, aspirina, statine).

I dati a supporto dell'efficacia di questi interventi 
sono limitati per le lungo-sopravviventi, e non vi sono precise raccomandazioni per il trattamento di pazienti che sviluppino scompenso cardiaco come conseguenza di trattamenti antitumorali, al di là delle indicazioni generali esistenti per i pazienti con disfunzione ventricolare. La durata del trattamento dello scompenso cardiaco da chemioterapia rimane incerta, anche se alcuni dati suggeriscono una lunga durata. ${ }^{66,76}$

È stato proposto l'utilizzo dell'acronimo ABCDE per la prevenzione cardiovascolare nelle pazienti survivors con carcinoma della mammella (A: awareness/aspirin; B: blood pressure; C: cholesterol/ cigarettes; D: diet/diabetes/dose chemo-radiotherapy; E: exerciselechocardiogram) ${ }^{77}$

La valutazione cardiologica integrata del paziente oncologico ha assunto un così importante rilievo da avviare una vera e propria disciplina la Cardio-oncologia al fine di poter gestire al meglio la paziente nel suo percorso di cura e, sicuramente studi di genere potranno essere pensati, disegnati e proposti in quest'ambito. $^{78,79}$

\section{Scompenso cardiaco, gender e infezione da SARS-CoV-2}

Non possiamo non inserire una riflessione anche su gender e infezione da SARS-CoV-2. I primi dati anche italiani disponibili, evidenziano un maggior rischio di ricovero in terapia intensiva per gli uomini, in presenza di comorbilità, come obesità, malattie renali e ipertensione arteriosa mentre nelle donne i due fattori preesistenti più significativi sono scompenso cardiaco ed obesità. ${ }^{80}$

Lo scompenso cardiaco, come comorbilità è presente nel $18,2 \%$ delle donne e nel $14,4 \%$ negli uomini, dei 63.573 deceduti nel nostro paese considerando i dati disponibili a dicembre $2020 .^{81}$

\section{Bibliografia}

1. Quaderni del Ministero della Salute "Il genere come determinante di salute" n.26 del 26 aprile 2016

2. Nichols M, Townsend N, Scarborough P, Rayner M. Cardiovascular disease in Europe 2014: epidemiological update. European Heart Journal, Volume 35, Issue 42, 7 November 2014, Pages 2950-2959, https://doi.org/10. 1093/eurheartj/ehu299

3. Loikas D, Wettermark B, von Euler M, et al. Differences in drug utilization between men and women: a crosssectional analysis of all dispensed drugs in Sweden. BMJ Open 2013;3: e002378. doi: 10.1136/ bmjopen2012-002378

4. Ministero della Salute "Piano l'applicazione e la diffusione della Medicina di Genere (in attuazione dell'art.3, comma1, Legge 3/ 2018)" www.salute.gov.it/imgs/C _17_pubblicazioni_2860_allegato.pdf · PDF file

5. Krakoff LR, Gillespie RL, Keith CF al. NIH Hyperten- sion recommendation from the National Eight Lawrence R. Joint Commitee Panel Members Raise Concern for Eldery Black and female Populations. Journal of the American College of Cardioogy VOL. 64, 2014 (4):395-401

6. Regitz Zagroseg V, Oertel-Prigione S, E. et al. Gender in cardiovascular diseases: impact on clinical manifestations. Eur Heart J 2016; 37:24-31

7. Virani SS, Alonso A, Benjamin EJ et al. Heart disease and stroke statistic - 2020 Update: A report from AHA. Circulation 2020;141(9):139-596

8. Heidenreich PA, Albert N M Allen LA et al. AHA Policy statement - Forecasting the Impact of Heart Failure in the United States: a Policy Statement From the American Heart Association. Circulation Heart Failur 2013;6: 216

9. Biagi P, Gussoni G, Iori I et al. Clinical profile and predictors of in-hospital outcome in patients with heart failure: the FADOI "CONFINE" Study. International Journal of Cardiology 2011;152: 88-94

10. Eisenberg E, Di Palo KE, IL. Sex differences in heart failure. Clin Cardiol.2018 41(2); 211-216

11. Meyer S, van deer Meer P, van Deursen V et al. Neurohormonal and clinical sex differences in heart failure. Eur Heart J 2013 Aug;34(32):2538-47. doi: 10.1093/ eurheartj/eht152. Epub 2013 May 10

12. Barcelona de Arellano MI, Pozdniakova S, Huhl AA et al. Sex differences in the aging human heart. Aging (Albany NY) 2019; 11:1918-33)

13. Olivetti G, Anversa P, Corradi D et. al. Gender differences and aging: Effects on the human heart. JACC 1995; 26:1068-1079

14. Bogle BM, Hongyan Ning MPH, Sanjay Mehrotra MS et al. Lifetime Risk for Sudden Cardiac Death in the Community. J Am Heart Assoc.2016; 5: e002398

15. Eileen M. Hsich, Ileana L. Piña, Heart failure in woman: a need for a prospective data. JACC 2009; 54:491

16. Kenchaiah S, Vasan RS. Heart failure in Woman - Insight from the Framingham heart study. Cardiovascular drug therapy (2015) 29:377-390

17. Eileen M. Hsich, Ileana L. Piña. Heart failure in women A need for prospective data. JACC 2009; 54:491

18. Chioncel O, P Collins S. Predictors of Post-discharge Mortality Among Patients Hospitalized for Acute Heart Failure. Eur J Heart Fail 2017; 19: 1574-78

19. Peddrotty DM, Jessup M. Frailty my name is Woman: syndrome of woman with heart failure with preserved ejection fraction. Circ Cardiovasc Qual outcomes, March 2015

20. Steven A, Mooser C et al. Atrial Fibrillation pattern and risk of subsequent stroke, heart failure and death in community. J Am Heart Assoc. 2013

21. Aune D, Sen A, Norat T et al. Body Mass Index, Abdominal Fatness, and Heart Failure Incidence and Mortality. Circulation 2016; 133:639-649

22. Golareth A, Louks E B et al. Healthy lifestyle and decreasing risk of heart failure in women. J am Coll Card 2014; 64: 1777-85

23. Cosentino F, Grant PJ, Abojans V et al. 2019 ESC guidelines on diabetes, pre-diabetes and cardiovascular diseases developed in collaboration with the EASD (European association for the study of Diabetes). http;// doi.org/10.1093/eurheartj/ehz828 14 nov 2019

24. Ponikowski P, Voors AA, Anker SD et al. 2016 ESC Guidelines for the diagnosis and treatment of acute and 
chronic heart failure: The Task Force for the diagnosis and treatment of acute and chronic heart failure of the European Society of Cardiology (ESC). Developed with the special contribution of the Heart Failure Association (HFA) of the ESC. EHJ 2016 doi: 10.1002/ejhf.592

25. Scott D, Salomon SD, McMurray JJVet alt. Angiotensin-Neprilysin Inhibition in Heart Failure with Preserved Ejection Fraction. NEJM 2019; 381:1609-1620

26. Chen YJ, Zang J, Wei-Jie Li, Chang Y et al. More Favorable response to Cardiac resyncronization in woman than in man. Circ Arrithm Electrophysiol.2014;7-807-815

27. Chatterjee NA, Borgquist R, Yuchao C et al. Increasing sex differences in the use of cardiac resynchronization therapy with or without implantable cardioverter-defibrillator. Euroheart J 2018 Apr 1; 39 (13) 1077 PMID: 28065904

28. Regitz-Zagrosek V, Seeland U. Sex and gender differences in clinical medicine. Handbook of experimental pharmacology, Springer Berlin Heidelberg .2012;33-22

29. Savonitto S. Aspetti di genere nelle malattie cardiovascolari. Il pensiero scientifico Ed. 2018

30. Santena BT, Ouwerk W, Tromp J et, al. Identifiying optimal doses of heart faiure medication in men compared with women: a prospective, observational study. Lancet 2019; 394: 1254-63

31. Hickey KT, Doering LV, Chen B et al. Clinical and gender differences in heart transplant recipiens in the NEW HEART study. Eur J cardiovasc Nurs 2017 Mar; 16(3) 222-228

32. HFA Practical guidance PPCM - European Journal of Heart Failure (2016) 18. 1096-1105 doi: 10.1002/ ejhf.586

33. Sliwa K, Hilfiker-Kleiner D, Mebazaa A et al. Heart Failure Association of the European Society of Cardiology Working Group on Peripartum Cardiomyopathy. Current state of knowledge on aetiology,diagnosis, management, and therapy of peripartum cardiomyopathy: a position statement from the Heart Failure Association of the European Society of Cardiology Working Group on peripartum cardiomyopathy. Eur J Heart Fail2010; 12:767-778

34. Hilfiker-Kleiner D, Sliwa K. Pathophysiology and epidemiology of peripartum cardiomyopathy. Nat Rev Cardiol 2014; 11:364-370

35. Hilfiker-Kleiner D, Haghikia A, Nonhoff J, Bauersachs J. Peripartum cardiomyopathy: current management and future perspectives. Eur Heart J 2015; 36:1090-1097

36. Patten IS, Rana S, Shaul S et al. Cardiac angiogenic imbalance leads to peripartum cardiomyopathy. Nature 2012; 485:333-3381

37. Hilfiker-Kleiner D, K, Podewski E et al A cathepsin Dcleaved $16 \mathrm{kDa}$ form of prolactin mediates postpartum cardiomyopathy. Cell 2007; 128:589

38. Duncker D, A, Konig T et al. Risk for ventricular fibrillation in peripartum cardiomyopathy with severely reduced left ventricular function: value of the wearable cardioverter/defibrillator. Eur J Heart Fail 2014; 16:1331-1336

39. Tibazarwa K, Lee G, Mayosi B et al.The 12-lead ECG in peripartum cardiomyopathy. Cardiovasc J Afr 2012; 23:1-8

40. Maisch B, Ruppert V, Pankuweit S. Management of fulminant myocarditis: a diagnosis in search of its etiology but with therapeutic options. Curr Heart Fail Rep 2014; 11: $166-177$

41. Mebazaa A, MB, Levy P et al, Recommendations on prehospital and early management of the hospital management of acute heart failure: a consensus paper from the ESC, ESEM and SAEM. Eur J Heart Fail 2015; 17: 544-558

42. Mebazaa A, Tolppanen H, Mueller C et al. Acute heart failure and cardiogenic shock: a multidisciplinary guidance. Intensive care Med 2016; 42: 147-163

43. HFA PRACTICAL GUIDANCE CCPP, European Journal of Heart Failure (2016) 18,1096-1105 doi:10.1002/ejhf.586

44. Templin C, Ghadri JR, Diekmann J et al. Clinical features and outcomes of Takotsubo (stress) cardiomyopathy. N Engl J Med 2015; 373:929-38.

45. Templin C, Hanggi J, Klein C et al. Altereted limbic and autonomic processing supports brain - heart axis in Takotsubo syndrome. Eur Heart J; 40: 1183-7. 2019).

46. Sharkey SW, Lesser JR, Zenovich AG et al. Acute and reversible cardiomyopathy provoked by stress in women from the United States. Circulation 2005; 111:472.

47. Ghadri JR, Wittstein IS, Prasad A et al. International expert consensus document on Takotsubo syndrome (Part I): clinical characteristics, diagnostic criteria, and pathophysiology. EurHeart J 2018; 39:2032-46).

48. Ghadri JR, Cammann VL, Napp LC et al. Differences in the clinical profile and outcomes of typical and atypical Takotsubo Syndrome: data from the International Takotsubo Registry. JAMA Cardiol 2016; 1:335-40

49. Uribarri A, Nunez-Gil IJ, Conty DA et al Short- and Long-Term Prognosis of Patients With Takotsubo Syndrome Based on Different Triggers: Importance of the Physical Nature https://oi.org/10.1161/JAHA.119. 013701

50. Ghadri JR, Kato K, Cammann VL et al. Long-term prognosis of patients with Takotsubo syndrome. J Am Coll Cardiol 2018; 72:874-82

51. El-Battrawy I, Lang S, Ansari U et al. Impact of concomitant atrial fibrillation on the prognosis of Takotsubo cardiomyopathy. Europace 2017; 19:1288-92

52. Di Vece D, Citro R, Cammann VL et al. Outcomes associated with cardiogenic shock in Takotsubo syndrome. Circulation 2019; 139:413-5

53. Lyon AR, Bossone E, Schneider B et al. Current state of knowledge on Takotsubo syndrome: a position statement from the Taskforce on Takotsubo Syndrome of the Heart Failure Association of the European Society of Cardiology. Eur J Heart Fail 2016; 18:8-27

54. Ghadri JR, Wittstein IS, Prasad A et al. International expert consensus document on Takotsubo syndrome (Part II): diagnostic workup, outcome, and management. Eur Heart J 2018; 39:2047-62

55. Almendro-Delia M, Nunez-Gil IJ, Lobo M et al. Shortand long-term prognostic relevance of cardiogenic shock in takotsubo syndrome: results from the RETAKO Registry. JACC Heart Fail 2018; 6:928-36

56. Madias JE. If channel blocker ivabradine vs. b-blockers for sinus tachycardia in patients with takotsubo syndrome. Int J Cardiol 2016; 223:877-8

57. Kim H, Senecal C, Lewis B et al. Natural history and predictors of mortality of patients with Takotsubo syndrome. Int J Cardiol 2018; 267:22-7. 
58. Dias A, Franco E, Koshkelashvili N et al. Antiplatelet therapy in Takotsubo cardiomyopathy: does it improve cardiovascular outcomes during index event? Heart Vessels 2016; 31:1285-90.

59. Kato K, Di Vece D, Cammann VL et al. Takotsubo recurrence: morphological types and triggers and identification of risk factors. J Am Coll Cardiol 2019; 73:982-4.

60. Zamorano JL,Lancellotti P, Munotz DR et al (2017), 2016 ESC Position Paper on cancer treatments and cardiovascular toxicity developed under the auspices of the ESC Committee for Practice Guidelines. Eur J Heart Fail, 19: 9-42

61. Lam CSP, Arnott C, Beale AL et al Sex differences in heart failure. Eur Heart J. 2019 Dec 14;40(47):3859-3868

62. Shukla PC, Singh KK, Quan A et al. BRCA1 is an essential regulator of heart function and survival following myocardial infarction. Nat Commun 2011; 2:593

63. Abdel-Qadir H, Austin PC, Lee DS et al. A populationbased study of cardiovascular mortality following earlystage breast cancer. JAMA Cardiol 2017; 2:88-93.

64. Davies N. Cancer survivorship: living with or beyond cancer. Cancer Nurs Pract 2009; 8(7):29-35

65. Guzzinati. F, Virdone S, De Angelis R et al. Characteristics of people living in Italy after a cancer diagnosis in 2010 and projections to 2020, BMC Cancer (2018) 18: 169, DOI 10.1186/s12885-018-4053-y)

66. Zamorano JL, Lancellotti P, Rodriguez Muñoz D et al. ESC Scientific Document Group. 2016 ESC Position Paper on cancer treatments and cardiovascular toxicity developed under the auspices of the ESC Committee for Practice Guidelines: The Task Force for cancer treatments and cardiovascular toxicity of the European Society of Cardiology (ESC). Eur Heart J. 2016 Sep 21;37(36):2768-2801.

67. Floyd JD, Nguyen DT, Lobins RL et al. Cardiotoxicity of Cancer Therapy. J Clin Oncol, 2005; 23: 7685-7696

68. Chen J, Long JB, Hurria A et al. Incidence of heart failure or cardiomyopathy after adjuvant trastuzumab therapy for breast cancer. J Am Coll Cardiol 2012; 60:2504-2512.

69. Haque R, Shi J, Schottinger JE et al. Cardiovascular disease after aromatase inhibitor use. JAMA Oncol 2016; 2:1590-1597.

70. Cheng YJ, Nie XY, Ji CC et al. Long-term cardiovascular risk after radiotherapy in women with breast cancer. J Am Heart Assoc 2017; 6 .

71. Travis LB, Ng A K, Allan JM et al. Second malignant neoplasms and cardiovascular disease following radiotherapy. J Natl Cancer Inst 2012; 104:357.
72. Darby SC, Ewertz M, McGale P et al. Risk of ischemic heart disease in women after radiotherapy for breast cancer. N Engl J Med 2013; 368:987.

73. Pudil R, Mueller C, Čelutkienè et al. Role of serum biomarkers in cancer patients receiving cardiotoxic cancer therapies: a position statement from the Cardio-Oncology Study Group of the Heart Failure Association and the Cardio-Oncology Council of the European Society of Cardiology. Eur J Heart Fail, 2020; 22: 1966-1983. https://doi.org/10.1002/ejhf.2017

74. Curigliano G, Dent S, Jordan K et al Management of cardiac disease in cancer patients throughout oncological treatment: ESMO consensus recommendations J Ann Onc 2020; 31 (2):171-190 https://doi.org/10.1016/j.annonc.2019.10.023 11-190, FEBRUARY 01, 2020

75. Armenian SH, Lacchetti C, Barac A et al. Prevention and Monitoring of Cardiac Dysfunction in Survivors of Adult Cancers: American Society of Clinical Oncology Clinical Practice Guideline. J Clin Oncol 2017; 35:893911

76. Shukla A, Yusuf SW, Daher I et al. High mortality rates are associated with withdrawal of beta blockers and ACE inhibitors in chemotherapy-induced heart failure. Circulation 2008;118: S797. Abstract 2942.

77. Montazeri K, Unitt C, Foody JM, et al. ABCDE steps to prevent heart disease in breast cancer survivors. Circulation. 2014;130: e157-e159.

78. Alizadehasl A, Amin A, Maleki M et al. Cardio-oncology discipline: focus on the necessities in developing countries. ESC Heart Fail. 2020 Oct;7(5):2175-2183.

79. Lyon AR, Dent S, Stanway S et al. Baseline cardiovascular risk assessment in cancer patients scheduled to receive cardiotoxic cancer therapies: a position statement and new risk assessment tools from the Cardio-Oncology Study Group of the Heart Failure Association of the European Society of Cardiology in collaboration with the International Cardio-Oncology Society. Eur J Heart Fail,2020; 22: 1945-1960 https://doi.org/10.1002/ ejhf. 1920

80. Iaccarino G, Grassi G, Borghi C et al. Gender differences in predictors of intensive care units admission among COVID-19 patients: The results of the SARSRAS study of the Italian Society of Hypertension. (2020) PLOS ONE 15(10): e0237297. https://doi.org/10.1371/journal.pone.0237297

81. Report COVID 19 - EPICENTRO - Istituto Superiore di Sanità Bollettino https://www.epicentro.iss.it/coronavirus/bollettino/Report-COVID19 16_dicembre 2020. 


\title{
Opportunità terapeutiche nel paziente con scompenso cardiaco cronico a frazione d'eiezione depressa
}

\author{
Alessandro Navazio, ${ }^{1}$ Massimo Piepoli, ${ }^{2}$ Giovanni Quinto Villani, ${ }^{2}$ Geza Halasz, ${ }^{2}$ Giovanni Tortorella ${ }^{3}$ \\ ${ }^{1}$ UOC Cardiologia Ospedaliera, Azienda USL di Reggio Emilia, IRCCS, Reggio Emilia; ${ }^{2}$ UOC Cardiologia, Ospedale Guglielmo \\ da Saliceto, Azienda USL di Piacenza; ${ }^{3}$ UOC Cardiologia, Ospedale Vaio, Azienda USL di Parma, Fidenza, Italia
}

\section{Terapia farmacologica}

\section{Introduzione}

In Italia sono oltre 1,2 milioni i pazienti con diagnosi di scompenso cardiaco (SC) un numero in continua crescita in massima parte dovuto al progressivo invecchiamento della popolazione ed al miglioramento delle tecniche di trattamento dell'infarto miocardico acuto. Nonostante l'evolversi della terapia farmacologica la prognosi rimane infausta con una mortalità che tocca il 50\% a 2 anni nei soggetti con $\mathrm{SC}$ avanzato ed un tasso di riospedalizzazione ad una anno del $31,2 \%{ }^{1}$

Il trattamento dello SC cronico è finalizzato a migliorare il quadro sintomatico, ed è indirizzato a prevenire la progressione della disfunzione cardiaca, a ritardare la comparsa dei sintomi e a ridurre la mortalità. Gli obiettivi invece consistono nel contrastare il rimodellamento cardiaco, l'attivazione neuroendocrina e citochinica, la ritenzione dei fluidi e la disfunzione renale.

Il primo obiettivo è raggiunto con l'impiego di farmaci ace-inibitori (ACE-I), $\beta$-bloccanti (b-bloccanti) e antagonisti dei mineral-corticoidi (MRA) che in numerosi studi hanno dimostrato di migliorare la sopravvivenza, i sintomi e prevenire le riospedalizzazioni. Sempre in questo contesto si inseriscono i sartani che rappresentano un'alternativa agli ace-inibitori quando questi non sono tollerati mentre un discorso a parte merita l'ormai nota combinazione tra Valsartan e sa-

Corrispondente: Giovanni Tortorella, UOC Cardiologia, Ospedale Vaio, Azienda USL di Parma, via Don F. Tincati 5, Fidenza (PR), Italia.

E-mail: gtortorella@ausl.pr.it

Articolo pubblicato secondo la Creative Commons Attribution NonCommercial 4.0 License (CC BY-NC 4.0).

${ }^{\circ}$ Copyright: the Author(s), 2021

Licensee PAGEPress, Italy

QUADERNI - Italian Journal of Medicine 2021; 9(2):e4 cubitril che alle proprietà vasolidilatatrici unisce un maggiore escrezione del sodio ed un maggiore impatto sul rimodellamento cardiaco. ${ }^{2}$ La terapia diuretica invece pur non avendo alcun impatto sulla mortalità 0 la progressione della malattia rappresenta un utile alleato nel prevenire l'accumulo di liquidi ed i sintomi ad esso connessi. Oltre ai farmaci citati negli ultimi anni sono entrati o stanno per entrare a far parte dell'armamentario del cardiologo diverse altre molecole; tra queste ricordiamo l'ivabradina, un inibitore dell' attività del pacemaker del nodo seno atriale in grado di ridurre la frequenza cardiaca nei pazienti in ritmo sinusale senza un'attività inotropa negativa, il dapaglifozin e l'empaglifozin, appartenenti alla classe degli inibitori del trasportatore sodio/glucosio tipo 2 (SGLT2) ed il vericiguat un farmaco che aumenta la sensibilità all'ossido nitrico che nello studio Victoria ha mostrato di ridurre significativamente sia la mortalità cardiovascolare che le ospedalizzazioni per SC. Nonostante l'efficacia delle summenzionate terapie nel ridurre la mortalità, i sintomi e la progressione della malattia, ancora esiste nella pratica clinica un ampio gap dovuto alla mancata applicazione delle linee guida. Infatti diversi studi hanno mostrato come più di $1 / 4$ dei pazienti con $\mathrm{SC}$ non ricevono terapia con ACE-inibitori/sartani, $\beta$-bloccanti, MRA o ARNI nonostante ne siano eleggibili. ${ }^{3}$ Allo stesso modo molto spesso anche quando queste terapie vengono prescritte sono spesso sottodosate il che si associa con un outcome peggiore visto che l'efficacia di queste classi di farmaci è dose dipendente.

\section{ACE inibitori e sartani}

È ormai noto come l'elemento fisiopatologico fondamentale nella progressione dello SC è rappresentato da una disregolazione del sistema renina angiotensina aldosterone (RAAS) la cui stimolazione incontrollata provoca accumulo di fluidi, vasocostrizione arteriosa periferica, ipertrofia dei cardiomiociti e fibrosi inter$\operatorname{stiziale}^{4}$ (Tabella 1). Per tale motivo gli ACE-I ed i sartani modulando negativamente l'attività di tale sistema rappresentano uno dei cardini del trattamento farma- 
cologico dello SC. A partire dagli anni novanta quando il primo farmaco della classe degli ACE-I è stato approvato per la cure dello SC una gran moltitudine di evidenze, tra cui recentemente una meta-analisi condotta su 12.763 pazienti con SC, ha confermato come l'inibizione del RAAS con ace inibitori o sartani riduce sensibilmente la mortalità, i ricoveri ospedalieri per insufficienza cardiaca e il re-infarto, indipendentemente dall'età, dal sesso e dall'uso concomitante di diuretici, aspirina e $\beta$-bloccanti. ${ }^{5}$ I benefici sono risultati evidenti qualsiasi fosse la funzione ventricolare sinistra basale. Gli effetti collaterali più frequenti associati a questa categoria di farmaci sono l'ipotensione, la sincope, l'insufficienza renale, l'iperkaliemia, l'angioedema e la tosse secca questi ultimi due sono dovuti all'accumulo di bradichinina nei tessuti ed a livello polmonare e costituiscono un effetto non dose dipendente appartenente a tutti gli ace-inibitori. In questi casi l'ACE-I deve essere sostituito con un Sartano che risulta meglio tollerato. Dal punto di vista pratico un'insufficienza renale moderata ed una pressione arteriosa relativamente bassa (creatininemia plasmatica fino a $250 \mu \mathrm{mol} / \mathrm{L}$ o $3 \mathrm{mg} / \mathrm{dL}$ e pressione sistolica $\geq 90 \mathrm{mmHg}$ ) non rappresentano controindicazioni al trattamento con ACE-I/Sartani mentre lo sono livelli di potassio plasmatico $>5,5 \mathrm{mmol} / \mathrm{L}$, la stenosi bilaterale delle arterie renali e l'angioedema verificatesi nel corso di una precedente terapia con ACE-I. Sarebbe preferibile utilizzare tra gli ACE-I quelli con più evidenze scientifiche derivanti da grandi trial quali l'enalapril, il ramipril ed il captopril quest'ultimo per la breve emivita dovrebbe essere preferito nei pazienti con valori pressori più bassi. In generale il dosaggio degli ACE-I va aumentato ogni 7-15 gg in base al profilo pressorio ed all'andamento dei dati di laboratorio quali gli indici di funzione renale e la potassiemia aumentando per i farmaci che richiedono due somministrazioni prima la dose serale e poi quella mattutina per scongiurare il rischio di ipotensione sintomatica. Nonostante l'ACE-inibizione sia indicata in tutti i pazienti con disfunzione sistolica ventricolare sinistra, indipendentemente dall'età, è tuttavia innegabile che gli anziani possono più spesso presentare controindicazioni assolute a questa forma di trattamento o manifestare effetti collaterali di rilievo nel corso dello stesso (peggioramento della funzione renale, squilibri elettrolitici, marcata risposta ipotensiva). È pertanto necessario che la titolazione dell'ACE-inibitore sia effettuata con particolare scrupolo in questi pazienti, partendo da bassi dosaggi che vanno raddoppiati ad intervalli di settimane sino a raggiungere quelli dimostratisi efficaci. Rispetto agli ACE-I i sartani antagonizzando l'attività del recettore angiotensina-I (AT-I) esercitano la loro azione ad un livello più basso della cascata del sistema RAAS non riducendo il catabolismo della bradichinina e prevenendo quindi il suo ac- cumulo che provoca, come detto, tosse ed angioedema. Nonostante questo vantaggio in termini di tollerabilità i sartani rappresentano un'alternativa agli ACE-I poiché il loro ruolo nel trattamento dello SCC non appare ancora chiarito in modo del tutto definitivo. Infatti, allo stato attuale, l'evidenza complessivamente emersa dai singoli trial e da valutazioni condotte su base metanalitica suggerisce che questi farmaci, pur dimostrandosi efficaci sui maggiori endpoint clinici, non sembrano fornire vantaggi significativi rispetto agli ACE-I. . $^{6} 7$

Sebbene sia gli ACE-I sia i sartani dovrebbero essere titolati fino alla massima dose tollerata in alcuni casi si possono accettare dosaggi minori come quando il raggiungimento di tale target terapeutico potrebbe precludere l'introduzione di altri farmaci quali b-bloccanti o MRA.

Per facilitare la compliance, spesso ridotta, dei pazienti anziani, può essere vantaggioso fare ricorso ad

Tabella 1. Lista e dosaggi di farmaci per lo scompenso cardiaco cronico a ridotta frazione di eiezione.

\begin{tabular}{|c|c|c|}
\hline (mg) & Dose iniziale (mg) & Dose Target \\
\hline \multicolumn{3}{|l|}{ ACE-I } \\
\hline Captopril & $6,25 \times 3$ & $50 \times 3$ \\
\hline Enalapril & $2,5-5 \times 2$ & $10-20 \times 2$ \\
\hline Lisinopril & $2,5-5 \times 1$ & $15-20 \times 2$ \\
\hline Ramipril & $1,25-2,5 \times 1$ & $5 \times 2$ \\
\hline Quinapril & $2,5-5 \times 1$ & $5 \times 2$ \\
\hline Fosinopril & $5 \times 1$ & $20 \times 1$ \\
\hline \multicolumn{3}{|l|}{$\beta$-bloccanti } \\
\hline Carvedilolo & $3,125 \times 2$ & $25 \times 2$ \\
\hline Bisoprololo & $1,25 \times 1$ & $5-10 \times 1$ \\
\hline Metoprololo & $12,5-25 \times 1$ & $50-100 \times 2$ \\
\hline Nebivololo & $1,25 \times 1$ & $10 \times 1$ \\
\hline \multicolumn{3}{|l|}{ Sartani } \\
\hline Candesartan & $4-8 \times 1$ & $32 \times 1$ \\
\hline Valsartan & $40 \times 2$ & $160 \times 2$ \\
\hline Losartan & $50 \times 1$ & $150 \times 1$ \\
\hline \multicolumn{3}{|c|}{ Diuretici dell'ansa } \\
\hline Furosemide & $20-40$ & $40-240$ \\
\hline Bumetanide & $0,5-1$ & $1-5$ \\
\hline Torasemide & $5-10$ & $10-20$ \\
\hline \multicolumn{3}{|l|}{ Diuretici tiazidici } \\
\hline Metolazone & 2,5 & $2,5-10$ \\
\hline Idroclorotiazide & 12,5 & $25-100$ \\
\hline Indapamide & 2,5 & $2,5-5$ \\
\hline
\end{tabular}


un agente somministrabile in dose unica giornaliera.

Infine pur essendo in genere meglio tollerati degli ACE-inibitori anche i sartani possono produrre degli effetti collaterali quali un peggioramento della funzione renale, iperpotassiemia ed ipotensione.

\section{$\beta$-bloccanti}

Un tempo considerati controindicati in caso di disfunzione ventricolare per il loro effetto inotropo negativo, i $\beta$-bloccanti oggi rappresentano insieme agli ACE-I la prima linea di trattamento dello SC in fase di stabilità (Tabella 1). Come detto l'attivazione neuroumorale è presente fin dalle prime fasi dell'insufficienza ventricolare ed è proporzionale alla sua gravità. I $\beta$-bloccanti possono interferire con tale processo a vari livelli: bloccano l'azione delle catecolamine e inibiscono l'attivazione simpatica attraverso il blocco dei recettori B1 e B2 nel cuore e nella midollare del surrene; ripristinano l'effetto inotropo positivo AMP ciclico-dipendente (ad esempio ripristinando la risposta inotropa agli inibitori delle fosfodiesterasi); diminuiscono le endoteline plasmatiche (che sono elevate nello scompenso), potenti vasocostrittori circolanti; aumentano l'attività vagale e riducono il rischio aritmico. ${ }^{8}$ Questi effetti si traducono, come recentemente evidenziato anche da una metanalisi che ha incluso 10 trial randomizzanti e 18.254 pazienti, in una riduzione della mortalità e delle ospedalizzazioni ed in un miglioramento significativo della sintomatologia e della capacità funzionale (incremento della durata di esercizio e dei metri percorsi al six-minute walk test), sebbene il massimo consumo di ossigeno non cambi o possa anche diminuire (probabilmente per l'effetto cronotropo negativo). Questi effetti sono additivi a quelli dovuti all'impiego degli ACE-I. ${ }^{9}$ Ad oggi i $\beta$ bloccanti più estesamente studiati e validati nell'ambito dello scompenso cardiaco a frazione di eiezione ridotta sono: carvedilolo, metoprololo succinato , bisoprololo e nebivololo.

In particolare il metoprololo possiede un maggiore effetto bradicardizzante ed è da preferirsi in quei pazienti tendenzialmente tachicardici ed ipotesi o in quelli con fibrillazione atriale.

Il carvediololo è stato il primo di questa classe di farmaci ad essere autorizzato per il trattamento dello $\mathrm{SC}$, è un antagonista non selettivo dei recettori adrenergici $(\beta 1, \beta 2, \alpha)$, privo di attività simpaticomimetica intrinseca e dotato di proprietà ancillari non condivise da nessun altro farmaco della stessa classe, quali l'azione antiossidante, antiproliferativa e calcioantagonista quando somministrato ad alte dosi. Le proprietà $\alpha$-bloccanti e la vasodilatazione periferica possono svolgere un ruolo benefico nelle fasi iniziali del trattamento riducendo il post carico cosi come assumono una notevole rilevanza clinica specialmente per i pazienti con $\mathrm{SC}$ che presentano fattori di rischio addizionali, quali l'ipertensione arteriosa ed il diabete associate peraltro ad una marcata disfunzione endoteliale.

Il nebivololo come il carvedilolo è un $\beta$-bloccante di terza generazione tuttavia a differenza di quest'ultimo possiede un'attività vasodilatatrice mediata dall'ossido nitrico ed ha un'azione selettiva sui recettori b1. Queste proprietà lo rendono il $\beta$-bloccante di scelta nei pazienti ipertesi ed in quelli con aumento delle pressioni di riempimento inoltre il suo effetto metabolico neutro lo rende preferibile nei pazienti diabetici.

Il bisoprololo invece si caratterizza per l'estrema maneggevolezza infatti la possibilità di assumerlo in mono somministrazione cosi come la disponibilità di multipli dosaggi lo rendono particolarmente utile nei pazienti in cui c'è bisogno di modulare la terapia in maniera molto accurata.

Nella pratica clinica l'inizio della terapia con $\beta$ bloccanti richiede il raggiungimento di un buon compenso emodinamico e l'assenza di segni di congestione. La dose deve essere aumentata gradualmente e cosi come per gli ACE-I dovrebbe essere raggiunto il massimo dosaggio tollerato in base ai valori pressori e di frequenza cardiaca ed al grado di compenso. Per ridurre il rischio di ipotensione sarebbe opportuno somministrare il b-bloccante ad un orario diverso rispetto all'ACE-I/sartano.

Riguardo alle controindicazioni non esiste alcuna controindicazione assoluta nei pazienti diabetici ma particolare cautela deve essere riservata a pazienti con un difficile controllo dei valori glicemici per la possibilità di peggiorare il controllo glicemico e per il rischio di incorrere in ipoglicemie paucisintomatiche. L'asma bronchiale non controllato e la BPCO di grado moderato-severo (stadio II-III con FEV1< al 50\% del predetto) rappresentano delle controindicazioni assolute al trattamento b-bloccante cosi come la presenza di ipotensione sintomatica, bradicardia spiccata $(\mathrm{FC}<50 \mathrm{bpm})$ e blocco AV di II e III grado o di I grado con $\mathrm{PR}>0,28 \mathrm{~s}$.

\section{Ivabradina}

Dalle linee guida del 2012 è entrata ufficialmente a far parte delle terapie raccomandate per lo SC anche l'ivabradina.

Tale farmaco è in grado di ridurre la frequenza cardiaca attraverso una inibizione selettiva della corrente pacemaker specifica che controlla la depolarizzazione spontanea del nodo del seno e regola la frequenza cardiaca. Gli effetti cardiaci sono specifici per il nodo del seno senza effetti sui tempi di conduzione intra-atriale, atrioventricolare o intraventricolare, né sulla contrattilità miocardica o sulla ripolarizzazione ventricolare. Lo studio SHIFT (randomizzato, in doppio cieco, controllato con placebo) ha valutato l'effetto dell'ivabradina somministrata fino al dosaggio tollerato $(\max 7,5 \mathrm{mg}$ 
$\mathrm{x} 2$ volte/die) in aggiunta alla terapia standard, in pazienti con sc cronico, $\mathrm{FE} \leq 35 \%$, con una frequenza cardiaca $\geq 70 \mathrm{bpm}$. Sono stati randomizzati 6.558 pazienti (età media 60,5 anni, $49 \%$ in classe NYHA II, $68 \%$ eziologia ischemica, solo $1 / 3$ dei pazienti con età $\geq 65$ anni). Dai risultati di questo studio che ha mostrato come l'utilizzo di ivabradina si associava ad una riduzione significativa sia delle mortalità per scompenso sia del numero delle ospedalizzazioni sono nate le indicazioni all'utilizzo di ivabradina. ${ }^{10}$ In particolare l'ivabradina può essere utilizzata in tutti quei pazienti con SC stabile in classe funzionale NYHA I-II con severa disfunzione ventricolare sinistra $(\mathrm{FE}<35 \%)$ in ritmo sinusale con $\mathrm{FC}>75 \mathrm{bpm}$ nonostante la terapia b-bloccante, oppure nei pazienti che non tollerano la terapia b-bloccante. L'ivabradina deve essere somministrata partendo da un dosaggio ridotto $(2,5 \mathrm{mg}-5 \mathrm{mg}$ due volte al giorno) che può essere incrementato dopo 15 giorni di trattamento, qualora la FC risulti ancora $>60$ bpm, alla dose massima di 7,5 mg 2 volte al giorno. I più comuni effetti collaterali di questa molecola sono rappresentati dalla bradicardia, dai fosfeni, dal blocco AV di primo grado e dall'extrasistolia ventricolare.

\section{Digitale}

Sebbene l'analisi della letteratura mostri che la digitale non è efficace nel ridurre la mortalità per scompenso e che nessun trial randomizzato abbia studiato gli effetti di questo farmaco in pazienti già in terapia b-bloccante, vari studi hanno dimostrato invece la sua utilità nel ridurre la sintomatologia e le ospedalizzazioni. ${ }^{11,12} \mathrm{Il}$ suo razionale di impiego è costituito dai pazienti con SC sintomatico avanzato (NYHA III-IV) e nei pazienti con $\mathrm{SC}$ e fibrillazione atriale il cui controllo delle $\mathrm{FC}$ non è raggiunto con la sola terapia $\mathrm{b}$ bloccante. Nella pratica clinica, salvo nei casi in cui occorre una rapida digitalizzazione, sembra essere ragionevole iniziare da una dose di $0,25 \mathrm{mg}$ in monosomministrazione. Dopo 1-2 settimane è bene controllare i livelli ematici del farmaco che devono essere compresi tra $0,7 \mathrm{ng}$ e $1,3 \mathrm{ng} / \mathrm{mL}$ con target di 1 $\mathrm{ng} / \mathrm{mL}$. Particolare cautela deve essere prestata ai pazienti anziani, a quelli con basso peso corporeo ed ai pazienti con ipotiroidismo ed insufficienza renale nei quali è ragionevole iniziare il trattamento con dosaggi più bassi $(0,125 \mathrm{mg} /$ die o $0,0625 \mathrm{mg} / \mathrm{die})$. Costituiscono controindicazioni relativi all'utilizzo della digitale il blocco AV di II grado, la malattia del nodo del seno, la cardiomiopatia ipertrofica ostruttiva e la stenosi aortica mentre i disturbi elettrolitici quali soprattutto l'ipocalcemia e l'ipokaliemia possono aggravare l'iperdosaggio digitalico che si manifesta con la comparsa di aritmie ipo-ipercinetiche sopraventricolari, astenia, malessere ed allucinazioni visive.

In questi casi risulta cruciale il controllo della digossinemia e del quadro elettrolitico ogni 3-6 mesi op- pure quando si apportano delle modifiche nel dosaggio $\mathrm{o}$ in caso di deterioramento della funzione renale.

\section{MRA}

Negli ultimi anni è divenuto chiaro come l'inibizione del RAAS ottenuta con ACE-I o con sartani non era completa, ma una quota di aldosterone continuava ad essere prodotta attraverso vie alternative (fenomeno dell'escape dell'aldosterone) supportando il persistere di un tasso elevato di mortalità e ri-ospedalizzazioni per scompenso nonostante una terapia che si riteneva ottimizzata. ${ }^{13}$ Partendo da questi presupposti si è fatta avanti l'ipotesi di utilizzare anche farmaci in grado di antagonizzare l'attività dell'aldosterone per ottenere un ulteriore miglioramento della prognosi. L'aldosterone, principale ormone mineralcorticoide prodotto dalle cellule della zona glomerulare della corteccia surrenalica, rappresenta l'effettore finale del RAAS ed i suoi recettori sono espressi sia a livello miocardico sia a livello endoteliale e coronarico. Attraverso questi recettori l'aldosterone è in grado di promuovere la fibrosi a livello cardiaco oltre ad esercitare un ruolo proaritmico e favorire, insieme al sovraccarico pressorio cronico, la dilatazione ventricolare sinistra. ${ }^{14} \mathrm{Gli}$ MRA sono in grado di antagonizzare gli effetti mediati dall'attivazione del recettore mineralcorticoide da parte dell'aldosterone attraverso un legame competitivo con il recettore stesso.

Diversi trial randomizzati hanno confermato il ruolo degli MRA nella terapia dello scompenso. In particolare si è dimostrato come nei pazienti con FE $<35 \%$, classe NYHA III e IV, già in terapia ottimizzata con ACE-I e diuretici e/o digossina e/o $\beta$-bloccanti l'aggiunta di un MRA producesse una significativa riduzione della mortalità e della necessità di ricovero per cause cardiache, del 30\% circa rispetto a placebo. Inoltre in questi pazienti si osservava una minore incidenza di ipokaliemia ed una maggiore riduzione della pressione arteriosa. ${ }^{15}$

I farmaci MRA attualmente disponibili sono: canrenone, canrenoato di potassio, spironolattone $\mathrm{e}$, di più recente introduzione, eplerenone. Essi presentano differenti caratteristiche farmacocinetiche che, se da un lato non condizionano una sostanziale differenza in termini di efficacia (tanto che si parla di effetto di classe degli MRA) dall'altro possono caratterizzare un diverso profilo di maneggevolezza e tollerabilità, spesso responsabili della sospensione del trattamento.

Lo spironolattone è un profarmaco che viene rapidamente metabolizzato a livello epatico e trasformato nei suoi metaboliti attivi (principalmente canrenone) mentre il canrenone e la sua forma salificata il canrenoato di potassio sono caratterizzati da un elevato assorbimento, non interferiscono con il citocromo P450 e sono eliminati principalmente per via renale e fecale. I principali effetti collaterali dello spironolattone e del 
canrenone oltre all'iperkaliemia sono secondari all'affinità del farmaco per altri recettori steroidei in particolare quelli per il testosterone e per l'estradiolo. Questa peculiarità può causare, in una percentuale non trascurabile di pazienti, effetti collaterali quali: ginecomastia, dolore mammario, impotenza e calo della libido. In questi casi può essere utilizzato l'eplerenone il quale essendo dotato di una maggiore selettività per il recettore dell'aldosterone non presenta tali effetti collaterali. Quest'ultimo, tuttavia, è meno potente dello spironolattone (attività antialdosteronica 40 volte minore dello spironolattone) e richiede dosi maggiori, con relativa perdita della selettività per il recettore dei mineralcorticoidi.

Dal punto di vista pratico la terapia, in assenza di controindicazioni (iperkaliemia $>5 \mathrm{mmol} / \mathrm{L}$; GFR $<30$ $\mathrm{mL} / \mathrm{kg} / 1,73 \mathrm{~m}^{2}$ ), può essere iniziata partendo da un dosaggio ridotto di spironolattone $(12,5 \mathrm{mg} / \mathrm{die})$. Tale dosaggio può essere ulteriormente ridotto, tramite somministrazione a giorni alterni, in pazienti più delicati come ad esempio negli anziani con tendenza all'iperpotassiemia spontanea o con un GFR $<50$ $\mathrm{mL} / \mathrm{kg} / 1,73 \mathrm{~m}^{2}$. Dopo circa 4 settimane il dosaggio può essere aumentato fino a $25-50 \mathrm{mg} /$ die. La potassiemia e la funzione renale andrebbero controllate frequentemente dopo 2-3 gg dalla prima dose, dopo una settimana e successivamente almeno mensilmente almeno nei primi mesi di terapia.

L'eplerenone dovrebbe essere riservato ai pazienti che manifestano ginecomastia o altra intolleranza alla spironolattone, partendo da un dosaggio di $25 \mathrm{mg} / \mathrm{die}$ anche eventualmente a giorni alterni e titolando la terapia fino a $50 \mathrm{mg} /$ die.

\section{ARNI (sacubitril/valsartan)}

Sacubitril/valsartan è la maggiore novità farmacologica degli ultimi 20 anni nell'ambito delle cure dei pazienti con SC cronico a funzione sistolica ridotta. Capostipite di una nuova classe di farmaci, gli ARNI (inibitori del recettore dell'angiotensina e della neprilisina), sacubitril/valsartan, tramite un'azione congiunta di inibizione della neprilisina e di antagonismo dell'angiotensina II, rappresenta una originale ed innovativa potenzialità terapeutica, per la sua attività di neuro-modulazione integrata del sistema dei peptidi natriuretici e del sistema renina-angiotensina.

Per comprendere il meccanismo d'azione di questo farmaco occorre approfondire il ruolo dei peptidi natriuretici nello SC.

I peptidi natriuretici, sono un gruppo di ormoni di natura peptidica, dei quali i più studiati sono il peptide natriuretico di tipo B (BNP) ed il peptide natriuretico atriale (ANP), la cui stimolazione e rilascio nell'organismo è strettamente legata all'effetto contro bilanciatorio causato dall'attivazione del sistema renina-angiotensina-aldosterone, dei cui risvolti clinici sull' organismo abbiamo già parlato e sui quali si basa gran parte della farmacoterapia dello SC. Nel circolo sistemico questi peptidi presentano un'emivita breve, ciascun peptide viene infatti degradato da una proteasi extracellulare nota come neprilisina (NEP) la quale limita considerevolmente i loro effetti benefici fisiologici. L'idea che alla base dell'ARNI è che l'organismo potrebbe aver bisogno di un'ulteriore quota di BNP circolante in corso di SC ed una strategia per aumentarne le concentrazioni consiste appunto nel bloccare l'enzima, la neprilisina, che ne compie la degradazione. Infatti in seguito alla somministrazione del farmaco, aumentano i livelli di ANP e BNP così come la diuresi, la natriuresi, la compliance ventricolare. L'utilizzo di un antagonista recettoriale dell'angiotensina, atto ad impedire il legame di essa con il recettore AT1, porta ad ulteriori effetti benefici quali una diminuita vasocostrizione, una minor ritenzione di sodio ed acqua, e una riduzione dell'ipertrofia cardiaca.

Nello studio PARADIGM-HF l'utilizzo di sacubitril/valsartan rispetto ad enalapril nei pazienti con SC e severa riduzione della funzione sistolica ha portato ad una riduzione del $20 \%$ del rischio di morte cardiovascolare e di ospedalizzazione per scompenso.

Inoltre il trattamento con tale farmaco si associava ad un aumento di sopravvivenza medio di 1,3 anni (in alcuni casi di oltre 2 anni) e ad una significativa riduzione dei livelli di aldosterone, NT-proBNP, troponina e metalloproteinasi confermando una riduzione dello stress e del danno di parete nonchè dei meccanismi profibrotici rispetto ai pazienti trattati con enalapril. ${ }^{16}$

Successivamente alla pubblicazione dei risultati dello studio PARADIGM-HF sia le linee guida europee che americane hanno inserito il farmaco con una raccomandazione di classe I, anche se con alcuni distinguo relativamente alla necessità di un pretrattamento con inibitori dell'enzima di conversione dell'angiotensina. Negli anni successivi diversi altri trial hanno confermato i dati dello studio PARDIGM$\mathrm{HF}$ anche nei pazienti con una funzione sistolica solo lievemente ridotta ed in quelli con SC di nuova insorgenza stabilizzato. ${ }^{17,18}$

Ad oggi il farmaco è indicato per il trattamento dello SC con severa riduzione della frazione di eiezione (FE $\leq 35 \%)$ in classe NYHA II-III e stabilità del quadro clinico. L'inizio della terapia dovrebbe avvenire dopo $36 \mathrm{~h}$ dalla sospensione di ACE-I o sartano e dovrebbe partire dalla dose iniziale di $24 / 26 \mathrm{mg}$ b.i.d. seguita da un raddoppio progressivo della dose ogni 2-4 settimane fino al raggiungimento della dose massima tollerata o della dose massima efficace che corrisponde a 97/103 mg b.i.d.

Visto che tale farmaco si associa ad ipotensione, angioedema ed iperkaliemia esso è contrindicato nei pazienti con valori di pressione arteriosa sistolica $<100 \mathrm{mmHg}$, nei pazienti con storia di angioedema ed 
in quelli con valori di potassio $>5,4 \mathrm{mmol} / \mathrm{L}$. Nell' ${ }^{\mathrm{i}}$ niziare la terapia bisogna prestare maggiore attenzione nei pazienti con un profilo clinico labile, quindi nei soggetti con tendenza ad ipotensione, insufficienza renale cronica, pregressi episodi di iperkaliemia e nei pazienti anziani. In questi casi è ragionevole una uptritation del dosaggio più graduale partendo anche da una dose minore tenendo presente che i benefici di sacubritil/valsartan si mantengono anche per dosaggi minori di 97-103 mg.

\section{Diuretici}

A differenza degli altri farmaci impiegati nello SC non esistono studi sull'effetto dei diuretici su mortalità e morbidità, l'impiego di questi farmaci infatti è volto soprattutto ad alleviare i sintomi legati alla congestione e conseguentemente a migliorare la capacità funzionale ${ }^{19}$ (Tabella 1). Di contro la terapia diuretica provoca una stimolazione significativa del sistema renina-angiotensina che a lungo termine potrebbe accelerare la progressione della malattia.

I diuretici maggiormente impiegati nella pratica clinica sono i diuretici dell'ansa ed i diuretici tiazidici i primi provocano una diuresi intensa e rapida mentre i secondi una diuresi moderata ma più prolungata nel tempo. Tra i diuretici dell'ansa i più utilizzati sono la furosemide e la torasemide, per tutti questi farmaci le curve dose risposta è lineare per cui all'aumentare della dose corrisponde un aumento della risposta diuretica. É pratica clinica iniziare con una dose bassa e incrementarla in caso di ritenzione idrica sotto controllo della funzione renale. La dose del diuretico è flessibile (con variazioni in aumento o in riduzione) e può essere modificata anche dal paziente opportunamente educato. Il sovradosaggio può provocare ipovolemia, ipotensione e compromissione renale

Tra i tiazidici invece i più utilizzati sono il metolazone e l'idrolorotiazide che inducono un modesto incremento della natriuresi, compreso fra 5 e $10 \%$ del carico filtrato, e la loro efficacia viene nettamente ridotta in presenza di insufficienza renale $(e G F R<30$ $\mathrm{mL} / \mathrm{kg} / 1,73 \mathrm{~m}^{2}$ ). Di solito l'utilizzo dei diuretici tiazidici è riservato ai pazienti con scompenso lieve o in quelli in cui sia necessario attuare il cosiddetto blocco sequenziale del nefrone per fenomeni di resistenza alla terapia con diuretici dell'ansa.

L'utilizzo ottimale dei diuretici non può prescindere dalla consensuale restrizione dell'apporto di liquidi e sali con la dieta (in particolare $<2-3 \mathrm{~g}$ /die di sodio) e dal controllo quotidiano del peso corporeo. L'obiettivo della terapia diuretica è il raggiungimento ed il mantenimento dello stato euvolemico; la dose di farmaco va aggiustata in modo che il peso corporeo del paziente si mantenga entro un'oscillazione di \pm 1 $\mathrm{kg}$ rispetto a quello ideale (cosiddetto peso asciutto).

Una conseguenza abituale della terapia diuretica è costituita dalla perdita di potassio. È dimostrato che la terapia diuretica mantenuta per lunghi periodi induce una progressiva diminuzione di potassio e magnesio plasmatici e dei muscoli scheletrici. L'aggiunta di farmaci antagonisti dell'aldosterone può ridurre questa perdita. Un'altra più temibile conseguenza è rappresentata dalla iponatriemia ipoosmolare, in parte dipendente anche dalla dieta iposodica; questa può essere corretta con la somministrazione di soluzioni saline, che permettano la ricostituzione del patrimonio sodico in precedenza depauperato

\section{Gliflozine (SGLT2 i)}

Il diabete e lo SC sono strettamente correlati: i pazienti con diabete hanno un aumentato rischio di sviluppare insufficienza cardiaca e quelli con insufficienza cardiaca sono a maggior rischio di sviluppare il diabete. La cardiomiopatia diabetica è caratterizzata da una precoce alterazione della funzione diastolica, accompagnata dallo sviluppo di ipertrofia e apoptosi dei cardiomiociti e fibrosi miocardica che esita nella maggior parte di casi in uno SC conclamato. ${ }^{20}$

Negli ultimi anni, si è assistito ad una vera e propria esplosione di nuove classi di farmaci antidiabetici con meccanismi di azione molto diversi tra loro che hanno dimostrato di ridurre non solo la glicemia ma anche le gravi complicanze cardiovascolari e renali ad essa correlate.

In particolar modo, una nuova classe farmacologica, le gliflozine, inibitori del cotrasportatore SGLT2, ha dimostrato di abbattere la progressione della nefropatia diabetica verso il grado più avanzato di insufficienza renale e nello stesso tempo di ridurre in maniera significativa il rischio di morte cardiovascolare e di ospedalizzazione nei pazienti affetti da SC. Tale classe di farmaci esplica la sua azione inibendo i co-trasportatori sodio-glucosio, SGLT1, che si trova principalmente nell'intestino tenue ed in maggior misura SGLT2, localizzato nel tubulo contorto prossimale e responsabile del riassorbimento del $90 \%$ del glucosio nel rene con conseguente glicosuria e riduzione della glicemia. Tali effetti si tradurrebbero a livello cardiaco con una riduzione sia del precarico, secondaria a natriuresi e diuresi osmotica, sia del postcarico attraverso la riduzione della pressione arteriosa (in media $4 \mathrm{mmHg}$ ), secondaria alla riduzione del sodio e al volume circolante.

Gli inibitori che sono stati approvati per il trattamento del Diabete mellito di tipo 2 sono empagliflozin, canagliflozin e dapagliflozin, che negli studi clinici hanno mostrato di ridurre significativamente gli eventi nei pazienti ad alto rischio cardiovascolare.

Il primo studio sugli SGLT2 inibitori è stato l'EMPAREG-CV outcome, uno studio di intervento con l'inibitore empagliflozin contro placebo e trattamento standard, effettuato in una coorte di pazienti con dia- 
bete mellito tipo 2 ad alto rischio cardiovascolare (con pregressi eventi o con multipli fattori di rischio $\mathrm{CV}$ ). Il risultati di questo studio sono stati stato sorprendenti: si è osservata una riduzione della morte cardiovascolare del $38 \%$, delle ospedalizzazioni per SC del $35 \%$ e della morte da tutte le cause del $32 \%$. L'effetto degli SGLT2 inibitori si osservava già nei primi 3 mesi di trattamento e non era mediato solo dalla riduzione della glicemia come suggerito dalla minima differenza in termini di HbAlc nei due bracci dello studio. ${ }^{21}$

Allo stesso modo nello studio CANVAS il trattamento con canaglifozin si associava nei pazienti con diabete di tipo II ad alto rischio cardiovascolare ad una riduzione del $14 \%$ dell'end-point primario costituito da infarto, stroke e mortalità cardiovascolare, e del $33 \%$ delle ospedalizzazioni per SC, dimostrando un effetto di classe degli SGLT2 inibitori. ${ }^{22}$

Tuttavia i risultati prima dello studio DAPA-HF e poi dello studio EMPEROR-reduced nel 2020 hanno esteso il ruolo di questa categoria di farmaci non solo nei pazienti con diabete ad alto rischio cardiovascolare ma anche nei pazienti non diabetici con SC. In particolare lo studio DAPA-HF ha valutato l'effetto del dapaglifozin rispetto a placebo nei pazienti con SC, diabetici e non diabetici, dimostrando nei due gruppi una significativa riduzione sia della mortalità cardiovascolare che per tutte le cause cosi come una riduzione delle ospedalizzazioni per scompenso. ${ }^{23}$ Alle stesse conclusioni è giunto un anno dopo lo studio EMPEROR-reduced che ha mostrato l'efficacia di empagliflozin rispetto a plecebo nel ridurre sia la mortalità che le ospedalizzazioni per SC nei pazienti con SC sia diabetici che non diabetici. ${ }^{24}$

Dal punto di vista pratico la dose utilizzata nei due trial è stata $10 \mathrm{mg} /$ die sia per il dapaglifozin nel DAPA-HG sia per l'empaglifozin nell'EMPEROR-reduced. Una dose minore dei due farmaci $(5 \mathrm{mg})$ può essere considerata in caso di peggioramento della funzione renale, ipotensione o tendenza all'ipoglicemia.

\section{Nitrati, idralazina e vericiguat}

La terapia di associazione idralazina (non disponibile in Italia) e nitrato risulta in un incremento della disponibilità di ossido nitrico con dilatazione sia del distretto venoso (nitrato) sia del distretto arterioso (idralazina) con conseguente riduzione del precarico e del postcarico. Questi effetti portano ad una riduzione sia delle pressioni di riempimento sia del rimodellamento inverso ventricolare che tuttavia non si sono tradotte in un chiaro beneficio in termini di mortalità e ospedalizzazioni per SC nei vari trials cha hanno studiato questi due farmaci, unica eccezione è rappresentata dai pazienti neri di razza africana nei quali questa associazione ha dimostrato una significativa riduzione della mortalità rispetto a placebo. ${ }^{25} \mathrm{Ad}$ oggi tale associazione è consigliata solo per un gruppo ristretto di pazienti ed in particolare nei pazienti neri con SC e severa riduzione della funzione sistolica sintomatici nonostante il trattamento con terapia convenzionale o in quelli che non tollerano gli ACE-I. Al di là delle raccomandazioni e del fatto che l'idralazina non è disponibile in Italia bisogna dire che il nitrato potrebbe essere indicato nei pazienti con SC su base ischemica ed in quelli con severo rigurgito mitralico nei quali la riduzione del precarico potrebbe mitigare l'impatto emodinamico dello stesso e conseguentemente sia la sintomatologia che la capacità funzionale.

Il fallimento dei trial sull'utilizzo dell' associazione idralazina e nitrato ha di recente rinnovato l'interesse su una molecola, il vericiguat, impiegata nel trattamento dell'ipertensione polmonare.

Il vericiguat è un farmaco che agisce stimolando la via ossido nitrico (NO)-guanilato ciclasi solubile (sGC)-guanosina monofosfato ciclico (cGMP) a livello endoteliale producendo una riduzione del tono vascolare arterioso. Tale via è alterata nello SC e conduce ad una minore biodisponibilità di $\mathrm{NO}$ endogeno, ad una minore affinità per $\mathrm{NO}$ della forma ossidata di sGC ed ad un deficit relativo di cGMP. Considerando che anche l'endocardio è sensibile all'ossido nitrico e che il processo di contrazione e rilasciamento miocardico è mediato dall'aumento delle contrazioni intracellulari di cGMP ne consegue che tale disregolazione ha effetti sulla performance ventricolare, sulla microcircolazione miocardica e sistemica, sulla funzione endoteliale e vascolare, sui processi infiammatori e sulla fibrosi. Partendo da questi presupposti fisiopatologici lo studio Victoria, condotto su una popolazione di 5050 pazienti con sc e $\mathrm{FE}<45 \%$, ha mostrato come l'utilizzo di tale farmaco sebbene non abbia ridotto in maniera significativa la mortalità per causa cardiovascolare era associato con una diminuzione delle ospedalizzazioni per $\mathrm{SC} .{ }^{26}$

\section{Omecamtiv mecarbil}

In passato l'approccio terapeutico allo scompenso cardiaco con funzione sistolica aveva contemplato farmaci che incrementassero la forza contrattile del miocardio, ma tutti gli studi sono stati fallimentari. Recentemente è stato testato un attivatore sperimentale della miosina cardiaca, l'omecamtiv mercabil, il primo di una nuova classe di inotropi progettati per agire sui meccanismi contrattili del miocardio, legandosi e reclutando più teste di miosina cardiaca per interagire con i filamenti di actina durante la sistole, senza aumentare le concentrazioni di calcio nei miociti intracellulari o il consumo di ossigeno miocardico. La miosina cardiaca è la proteina motoria citoscheletrica nella cellula del muscolo cardiaco che è direttamente responsabile della conversione dell'energia chimica nella forza meccanica risultante in contrazione cardiaca. Questa molecola è stata testata nello studio GA- 
LACTIC-HF, multicentrico, randomizzato, doppio cieco controllato con placebo, in pazienti con scompenso cardiaco cronico con ridotta frazione di eiezione $(\leq 35 \%)$. I risultati dello studio sono stati presentati all'AHA 2020 e pubblicati sul NEJM il 13/11/2020 ed hanno mostrato un modesto guadagno in termini dell'end point primario dello studio rappresentato da riduzione della quantità di tempo prima della morte cardiovascolare o della necessità di un trattamento per insufficienza cardiaca. Il beneficio era modesto con una riduzione assoluta del $2,1 \%$ e una riduzione del rischio relativo dell' $8 \%$ su una mediana di follow up di 22 mesi. Inoltre non vi sono stati benefici nel gruppo trattato con omecamtiv mercabil sui singoli componenti dell'end point primario quando considerati singolarmente. Alla luce di questi risultati sarà da definire se e dove allocare questa terapia. ${ }^{27,28}$

\section{Terapia elettrica}

L'utilizzo dei dispositivi impiantabili di stimolazione ha ampiamente rivoluzionato la terapia cardiologica dei pazienti con scompenso cardiaco entrando nelle Linee Guida delle Società Nazionali ed internazionali nell'ambito del trattamento delle bradi-tachiaritmie e del miglioramento della contrattilità miocardica. ${ }^{29,30}$

\section{Modalità di pacing in pazienti con scompenso cardiaco}

È ormai ampiamente dimostrato come la stimolazione ventricolare destra apicale comportando una attivazione dissincrona debba essere considerata un potenziale causa di sviluppo/peggioramento di una disfunzione ventricolare sinistra. ${ }^{31,32}$ In alternativa sono stati proposti siti di stimolazione alternativi (setto ventricolare destro, tratto di efflusso ventricolare destro) per ovviare ai suoi potenziali effetti negativi. Tali approcci hanno evidenziato una influenza positiva sulla frazione di eiezione in pazienti con dimostrata disfunzione $(\mathrm{FE}<45 \%)$ senza tuttavia modificare la qualità di vita, la capacità funzionale e la mortalità. ${ }^{33}$ In considerazione del fatto che più del $50 \%$ dei pazienti con $\mathrm{SC}$ e disfunzione ventricolare sinistra presenta un ritardo di conduzione intraventricolare è stata introdotta in terapia la stimolazione biventricolare (CRT) allo scopo di normalizzare la attivazione miocardica e conseguentemente la funzione cardiaca. ${ }^{34-36}$ Tale modalità di stimolazione in pazienti con blocco di branca sinistro ha dimostrato di ridurre la mortalità e di migliorarne la capacità funzionale, ${ }^{37}$ il raggiungimento dell'obiettivo terapeutico è in gran parte correlato alla effettiva riduzione del QRS stimolato. ${ }^{38}$ Sulla base dei dati di letteratura le linee guida ESC raccomandano l'uso della CRT (Classe I A-B) nei pazienti con fra- zione di eiezione $<35 \%$, Blocco di branca sinistro (durata $>150 \mathrm{msec}$ A, 130-149 msec B) in terapia medica ottimale o che necessitino di stimolazione ventricolare permanente per una bradi aritmia. ${ }^{29,30}$ Esistono tuttavia condizioni cliniche (impossibilità anatomica di corretta stimolazione sinistra, P-R lungo e QRS stretto, ritardi di attivazione ventricolare non a tipo Blocco di Branca sinistro) in cui una CRT classica può non essere la scelta ottimale. Come alternative sono state proposte sedi alternativa di posizionamento dei cateteri allo scopo di perseguire una migliora re sincronizzazione: i) la stimolazione hisiana (non inferiore alla CRT classica);39 ii) la stimolazione ventricolare sinistra (maggiore capacità di accorciamento della durata del QRS); ${ }^{40}$ iii) la stimolazione ventricolare destra bifocale (in soggetti con Blocco di Branca destro); ${ }^{41}$ iv) la stimolazione sequenziale del fascio di His e del ventricolo sinistro attraverso diramazioni del seno coronarico o con l'aggiunta della stimolazione ventricolare destra (TRIPLE). ${ }^{42}$

Nell'ambito del trattamento dei pazienti con Insufficienza Cardiaca moderata è stata inoltre valutata la modulazione della contrattilità in cui la stimolazione elettrica viene applicata al muscolo durante il periodo refrattario assoluto. In questa fase del ciclo cardiaco i segnali elettrici non possono innescare nuove contrazioni, quindi questo tipo di stimolazione è noto come stimolazione non eccitatoria. Uno studio preliminare ha precedentemente dimostrato che la modulazione della contrattilità cardiaca può essere sicura ed efficace in quei pazienti che non hanno risposto alla terapia di resincronizzazione. ${ }^{43}$ Le linee guida emesse dalla Società Europea di Cardiologia menzionano la terapia di modulazione della contrattilità cardiaca come opzione terapeutica da considerare in un gruppo selezionato di pazienti. ${ }^{29}$

\section{Il defibrillatore automatico}

È noto come in una alta percentuale di pazienti con insufficienza cardiaca a bassa frazione di eiezione il decesso sia dovuto alla morte improvvisa, correlata spesso ad aritmie iper/ipocinetiche. La terapia medica ottimale volta al rallentamento della progressione della malattia non è stata tuttavia in grado di ridurre la incidenza di tale evento che ha ricevuto una parziale risposta dall'impianto del defibrillatore impiantabile (ICD). Le attuali Linee Guida $\mathrm{ESC}^{1}$ raccomandano l'impianto di un ICD in prevenzione Primaria in tutti i pazienti con una Insufficienza Cardiaca sintomatica (Classe NYHA II-III) con una frazione di eiezione $<35 \%$ dopo almeno tre mesi di terapia medica ottimale e con una aspettativa di vita di almeno un anno. I pazienti candidabili sono quelli con Cardiopatia Ischemica ad evoluzione dilatativa (escluso il paziente con Infarto Miocardico acuto $<40$ dì) o con miocardiopatia dilatativa primitiva (Classe I A-B). L'uso dell'ICD è 
inoltre raccomandato in prevenzione secondaria in quei pazienti che evidenziano aritmie ipercinetiche ventricolari con instabilità emodinamica (Classe I A). L'utilità di tale impostazione è stata recentemente confermata dallo studio EURO-CERT-ICD che ha evidenziato una riduzione del $27 \%$ della mortalità totale in un follow-up medio di $2,4 \pm 1,1$ anni $^{16,44}$ sia nei pazienti con cardiopatia dilatativa di origine ischemica che primitiva. Il beneficio atteso è tuttavia non significativo nei pazienti diabetici ed in quelli con età superiore ai 75 anni. Tale osservazione conferma il dato noto della scarsa efficacia in gruppi di pazienti con severe comorbidità. ${ }^{7,45}$ L'utilizzo del defibrillatore impiantabile sottocutaneo deve essere limitato ai pazienti che non necessitano di stimolazione ventricolare per bradi aritmia o che non hanno indicazione alla CRT. Il defibrillatore automatico indossabile viene invece raccomandato per pazienti con una aspettativa di vita inferiore ad 1 anno od in quelli in cui è ipotizzabile un miglioramento della funzione contrattile ma con un basso livello di evidenza (Classe II B). ${ }^{29}$ L'utilizzo dell'ICD è sconsigliato in pazienti in Classe NYHA IV con sintomi severi refrattari alla terapia medica, senza indicazioni alla CRT, all'impianto di un sistema di assistenza ventricolare o ad un trapianto cardiaco (Classe III C).

\section{Assistenza ventricolare nell'insufficienza cardiaca avanzata}

I progressi della terapia di resincronizzazione ventricolare uniti alla terapia medica ottimale hanno notevolmente migliorato la prognosi e la qualità di vita nei pazienti affetti da insufficienza cardiaca. Tuttavia il 4-5\% di questi pazienti giunge a condizioni di scompenso cardiaco avanzato e circa 1' $1,5-2 \%$ presenta quadri di scompenso refrattario. I pazienti con scompenso cardiaco avanzato hanno una mortalità del $35 \%$ a 1 anno e del $69 \%$ a 2 anni. Quelli con scompenso cardiaco refrattario presentano una mortalità a 1 anno del $50-75 \%$ e a 2 anni superiore al $90 \% .{ }^{46,47}$

Il trapianto di cuore (TC) rimane il trattamento gold standard per l'insufficienza cardiaca avanzata refrattaria alla terapia medica ed elettrica; è cioè ancora la terapia che assicura la migliore sopravvivenza a lungo termine e il minor tasso di complicanze nel breve termine. La percentuale di pazienti che possono beneficiare del trapianto cardiaco è limitata da un lato dal ristretto numero di donatori, dall'altro dall'inapplicabilità di tale procedura in pazienti anziani $(>65$ anni) o con comorbilità e condizioni cliniche che controindicano il trapianto stesso. Negli ultimi decenni al trapianto di cuore si sono affiancate nuove strategie terapeutiche tecnologicamente avanzate che possono oggi essere considerate una nuova e reale opzione terapeutica per il paziente affetto da scompenso cardiaco avanzato/terminale. Parliamo dei dispositivi di assistenza ventricolare (ventricular assist device, VAD), pompe meccaniche in grado di supplire la funzione di un cuore compromesso in maniera più o meno irreversibile ristabilendo un flusso ematico il più possibile simile a quello fisiologico. ${ }^{48}$

\section{Indicazioni e selezione dei pazienti}

L'assistenza meccanica al circolo trova indicazione quando il trattamento intensivo convenzionale, farmacologico e non, non è più in grado di assicurare condizioni cliniche ed emodinamiche accettabili. In Tabella 2 sono riportate le caratteristiche di un paziente potenzialmente eleggibile ad impianto di un VAD secondo le indicazioni ESC del 2016. ${ }^{29}$ Per anni è risultato alquanto dibattuto l'argomento su quali strumenti utilizzare per un corretto triage dell'insufficienza cardiaca terminale utile a definire il timing di impianto di un VAD. Le classificazioni più utilizzate per l'insufficienza cardiaca, quella NYHA (funzionale per classi da I a IV) e quella sintomatologica delle più recenti linee guida sull'argomento (stadi da "A" a "D"), inquadrano tutti i pazienti potenzialmente candidabili ad impianto di un VAD nel gruppo a maggior gravità, non tenendo conto che tra i pazienti con insufficienza cardiaca avanzata esistono sottogruppi molto diversi per caratteristiche cliniche. Con l'istituzione del registro INTERMACS, ${ }^{49}$ è stata creata un ulteriore classificazione degli stadi dell'insufficienza cardiaca avanzata per livelli di stabilità/instabilità clinica con possibilità di confronto anche in termini prognostici (Tabella 3). Tale classificazione prevede 7 profili clinici: dal livello 1 che caratterizza il paziente più severo, in shock cardiogeno, fino al livello 7 che include i soggetti con insufficienza cardiaca avanzata clinicamente stabili. Questa metodologia di classificazione si è dimostrata molto duttile ed adattabile al-

Tabella 2. Pazienti potenzialmente eleggibili ad impianto di VAD. ${ }^{29}$

Pazienti con sintomi severi da $>2$ mesi nonostante terapia medica ottimale (farmacologica e non) e più di uno dei seguenti:

- FE ventricolare sx $<25 \%$ e, se misurato, peakVO $\mathrm{O}_{2}<12 \mathrm{~mL} / \mathrm{kg} / \mathrm{min}$

- Ospedalizzazioni per insufficienza cardiaca nei precedenti 12 mesi senza chiare cause precipitanti

- Dipendenza da inotropi ev

- Progressiva disfunzione d'organo (renale ed epatica) dovuta a riduzione della perfusione non ad inadeguate pressioni di riempimento ventricolare (PCWP $>20 \mathrm{mmHg}$ e $\mathrm{SBP}<80-90 \mathrm{mmHg}$ o $\mathrm{CI}<2 \mathrm{~L} / \mathrm{min} / \mathrm{m}^{2}$ )

- Assenza di severa disfunzione ventricolare destra insieme ad insufficienza tricuspidalica severa 
l'evoluzione della patologia. Essa considera anche il concetto di evento clinico in grado di modificare in senso peggiorativo il livello di compromissione del paziente. Ad esempio un paziente inquadrabile in classe 4 qualora richiedesse frequenti ospedalizzazioni (frequent flyer) verrebbe inquadrato nel profilo 3. L'analisi e il confronto delle curve di sopravvivenza in base al profilo clinico INTERMACS del paziente ha confermato le potenzialità di questa classificazione come strumento utile per decidere il timing dell'impianto. ${ }^{50}$ Le curve di mortalità di pazienti sottoposti ad impianto primario di assistenza sinistra ${ }^{51}$ hanno dimostrato come la sopravvivenza, stratificata per livelli INTERMACS, sia peggiore nei pazienti sottoposti ad impianto ad un livello 1, e ciò in gran parte a causa della mortalità precoce di questi pazienti, già particolarmente compromessi prima dell'operazione. I risultati migliori (sopravvivenza a 12 mesi intorno

Tabella 3. Livelli INTERMACS: descrizione dei profili.

Descrizione dei profili
Livello 1: Shock cardiogeno critico
Pazienti con ipotensione severa nonostante rapido aumento del supporto inotropo, critica ipoper-
fusione d'organo, spesso associata a peggioramento dell'acidosi e/o dei livelli di lattati
Livello 2: Declino progressivo
Pazienti con declino funzionale nonostante supporto inotropo e.v., può manifestarsi con peggiora-
mento della funzione renale, declino dello stato nutrizionale, impossibilità di ripristinare un ade-
guato equilibrio volemico. Descrive, inoltre, lo stato di declino di quei pazienti intolleranti alla
terapia con inotropi (e.g. a causa di aritmie)

\section{Livello 3: Stabile ma dipendente da inotropi} dei sintomi con supporto inotropo continuo e.v. (o con dispositivo supporto circolatorio temporaneo o entrambi), ma con ripetuti fallimenti dei tentativi di svezzamento dal supporto inotropo/meccanico, a causa del ripresentarsi di ipotensione severa o disfunzione renale

\section{Tempistica di intervento}

Necessario intervento definitivo entro
poche ore

Necessario intervento definitivo entro pochi giorni
Pazienti con stabilità della pressione arteriosa, della funzione d'organo, dello stato nutrizionale e

\section{Livello 4: Sintomi a riposo}

Il paziente può essere stabilizzato in condizioni prossime all'euvolemia, ma presenta quotidianamente sintomi da congestione a riposo o durante le normali attività quotidiane. Generalmente il dosaggio del diuretico si attesta su valori molto alti. Devono essere adottati sia una gestione più intensiva che strategie di monitoraggio, che, in alcuni casi, possono compromettere la compliance e di conseguenza il risultato, con qualsiasi terapia. Alcuni pazienti possono fluttuare tra il livello 4 e 5

\section{Livello 5: Intolleranza all'esercizio}

Paziente asintomatico a riposo e durante le normali attività quotidiane, ma incapace di intraprendere qualsiasi altra attività, vivendo prevalentemente in casa. È un paziente asintomatico a riposo, ma può avere comunque un sovraccarico di volume refrattario alla terapia diuretica, e spesso associato a insufficienza renale.
Intervento definitivo elettivo nell'arco di settimane o pochi mesi

Intervento definitivo elettivo nell'arco di settimane o pochi mesi

Urgenza variabile, dipende dallo stato nutrizionale, dalla disfunzione d'organo e dalla tolleranza all'esercizio

\section{Livello 6: Limitazione all'esercizio}

Il paziente, scevro da sovraccarico di volume, è asintomatico a riposo, durante le normali attività quotidiane e le piccole attività fuori casa, ma diventa rapidamente sintomatico dopo pochi minuti di attività fisica significativa. L'attribuzione ad un sottostante deficit cardiaco richiede una attenta Variabile, dipende dallo stato nutrizionale, dalla disfunzione d'organo e dalla tolleranza all'esercizio misurazione del picco di consumo di ossigeno, e, in alcuni casi, una valutazione emodinamica, a conferma della severità della disfunzione cardiaca

\section{Livello 7: NYHA III avanzata}

Questo livello si apre a future nuove descrizioni più precise. Include pazienti senza attuali o recenti Trapianto o supporto circolatorio potrebepisodi di instabilità e ritenzione di fluidi, che vivono asintomatici e sono in grado di tollerare at- bero non essere indicati al momento tività fisiche significative fino all'attività fisica lieve

\section{Eventi in grado di aggravare il profilo INTERMACS}

TCS - temporary circulatory support (supporto circolatorio temporaneo)

Include contropulsazione aortica (IABP), ECMO, TandemHeart, Levitronix, BVS5000 o AB5000, Applicabile ai Livelli 1, 2, 3 di pazienti e Impella. Si applica solo a pazienti ricoverati in ospedale ospedalizzati

\section{A-Aritmia}

È applicabile a qualsiasi profilo. Tachi-aritmia ventricolare ricorrente che recentemente ha sostanzialmente contribuito alla compromissione clinica del paziente. Include frequenti interventi appropriati dell'ICD o la necessità di defibrillazione elettrica esterna, solitamente più di 2 volte a settimana.

\section{FF - Frequent flyer} È applicabile solo a pazienti non ospedalizzati, descrivendo quei pazienti che richiedono frequenti
rivalutazioni cliniche in emergenza o ospedalizzazioni per terapia diuretica, ultrafiltrazione, o infusione temporanea di terapia vasoattiva livello 7 
all' $82 \%$ ) si sono invece ottenuti nei pazienti con un livello 3 , cioè in condizioni cliniche stabili seppur in terapia con inotropi. ${ }^{52}$

\section{Quale assistenza ventricolare}

I livelli di assistenza al circolo possono essere classificati in base a: i) tipo di flusso generato: flusso pulsatile o continuo; ii) sede di impianto: paracorporeo, intracorporeo; iii) ventricolo assistito: monoventricolare (esclusivamente sinistro, più raramente destro), biventricolare (supporto contemporaneo di entrambi i ventricoli); iv) potenziale durata del sistema di assistenza : breve (da qualche ora a pochi giorni); media (dalle 2 alle 4 settimane); lunga (dalle 4 settimane ad un tempo potenzialmente indeterminato).

I sistemi di assistenza ventricolare comunemente utilizzati constano di una cannula di afflusso (che porta il sangue dal ventricolo sinistro o dall'atrio destro al ventricolo artificiale); di una pompa (o ventricolo artificiale) a flusso continuo o pulsatile e di una cannula di efflusso (che convoglia il sangue dal ventricolo artificiale ad una grande arteria solitamente aorta ascendente più raramente aorta discendente toracica).

I fattori che condizionano la scelta del device nel singolo paziente sono lo scopo dell'assistenza e la sua durata prevista.

Gli scenari più comuni sono:

- condizioni caratterizzate da un quadro emodinamico estremamente grave tuttavia con possibilità di recupero della funzione cardiaca nel breve periodo: miocardite acuta fulminante, shock cardiogeno da altre cause (ad es cardiomiopatia post partum, infarto miocardico acuto), sindrome post pericardiotomica. In questi casi si prevedono assistenze di breve durata $(<1-2$ settimane) e si ricorre prevalentemente a device che richiedono una invasività chirurgica ridotta. I device più adatti sono: i) l'ECMO (extracorporeal membrane oxygenation), ${ }^{53}$ tecnica di assistenza cardiorespiratoria molto utilizzata in queste situazioni, efficace, il cui impianto avviene solitamente tramite incannulazione dei vasi femorali quindi la necessità di aprire il torace; ii) le pompe microassiali intravascolari tipo Impella. ${ }^{54}$ Questi device, per la minore invasività e per il costo nettamente inferiore rispetto ai sistemi di più lunga durata, trovano sempre più spesso indicazione come bridge to decision. In acuto infatti è spesso difficile predire come evolverà la situazione clinica dopo il supporto meccanico. Una assistenza a breve termine potrà aiutare il clinico a valutare la strategia futura che potrà contemplare un recupero anche solo parzialmente completo, la necessità di un trapianto cardiaco urgente o l'impianto di un VAD permanente (sia come ponte al trapianto che come destination therapy).

- ponte al trapianto cardiaco: è la condizione più frequente e dal momento che la disponibilità di un donatore non è prevedibile, è necessario utilizzare sistemi in grado di sostenere il circolo per un periodo di tempo prolungato, soprattutto in quei pazienti che per motivi clinici potrebbero incontrare più difficolta di altri nella disponibilità di un donatore idoneo (taglia corporea, gruppo sanguigno, iperimmunizzazione). L'impianto di un sistema di assistenza ventricolare in un candidato a trapianto cardiaco viene preso in considerazione quando la gravità del quadro clinico fa ritenere che il prolungarsi dell'attesa di un donatore comporti un rischio di morte o deterioramento irreversibile superiore al rischio dell'impianto di VAD. L'assistenza ventricolare può dare a questi pazienti l'opportunità di attendere il trapianto in condizioni migliori mantenendo la funzionalità epatorenale, l'autonomia funzionale ed un adeguato stato nutrizionale. $\mathrm{La}$ possibilità di dimettere i pazienti in assistenza consente un ulteriore miglioramento della qualità della vita ed ha permesso in alcuni casi anche la ripresa dell'attività lavorativa.

- destination therapy e bridge to candidancy: in caso di insufficienza cardiaca refrattaria in pazienti non candidabili al trapianto (per età avanzata, pregressa/recente patologia neoplastica, multiple comorbilità) si prospetta una assistenza ventricolare di lunga durata come trattamento definitivo o alternativo al trapianto. Vengono utilizzati device totalmente impiantabili per garantire la mobilizzazione o la completa autonomia del paziente. Lo studio REMATCH $^{55}$ ha confrontato la sopravvivenza, gli eventi clinici e la qualità di vita in due gruppi di pazienti non idonei al trapianto, con caratteristiche di gravità dell'insufficienza cardiaca (ospedalizzati, classe NYHA IV, frazione d'eiezione ventricolare sx $\leq 25 \%$, dipendenza da inotropi oppure con un consumo massimo di ossigeno $\leq 14 \mathrm{~mL} / \mathrm{kg} / \mathrm{min}$ ) condizionanti una cattiva prognosi a breve termine, randomizzati a terapia medica o a impianto di LVAD (tipo HeartMate). La sopravvivenza dei 129 pazienti arruolati è stata significativamente maggiore nei pazienti trattati con LVAD rispetto a quelli in terapia medica ( $52 \%$ vs $25 \%$ e $23 \%$ vs $8 \%$ ad $1 \mathrm{e}$ 2 anni rispettivamente), pur a fronte di una elevata incidenza di complicanze correlate al VAD come infezioni, problemi neurologici, malfunzionamento del device. Risultati ancora più incoraggianti sono derivati dai dati del registro I pubblicati nel 2010. Qui la sopravvivenza dei pazienti sottoposti ad impianto primario di VAD si attesta intorno al $74 \%$ nell'intera popolazione e raggiunge $1^{\prime} 82 \%$ nei pazienti con livello INTERMACS 3 al momento dell'impianto.

- bridge to recovery: durante supporto prolungato con LVAD come ponte al trapianto in una (piccola) 
quota di pazienti è stato osservato un miglioramento progressivo della funzione ventricolare sinistra che ha consentito in alcuni casi l'espianto del device, con persistenza di una funzione ventricolare sx accettabile ( $\mathrm{FE}>40 \%)$ per periodi di tempo anche più o meno prolungati. ${ }^{56} \mathrm{Si}$ ritiene che l'unloading progressivo determinato dall'assistenza, la normalizzazione del regime emodinamico e la riduzione dell'attività simpatica possano essere responsabili del reverse remodeling osservato in corso di assistenza. Solitamente i pazienti nei quali è posssibile rimuovere il device sono affetti da cardiomiopatia dilatativa idiopatica o da miocardite ed hanno una storia più breve di malattia.

\section{Bibliografia}

1. Maggioni AP, Orso F, Calabria S, et al. The real-world evidence of heart failure: findings from 41413 patients of the ARNO database. Eur J Heart Fail. 2016; 18:402-10.

2. Murphy SP, Ibrahim NE, Januzzi JL Jr. Heart Failure With Reduced Ejection Fraction: A Review. JAMA. 2020; 324:488-04

3. Greene SJ, Butler J, Albert NM, et al. Medical therapy for heart failure with reduced ejection fraction: the CHAMPHF registry. J Am Coll Cardiol 2018;72:351-66

4. Sayer G, Bhat G. The renin-angiotensin-aldosterone system and heart failure. Cardiol Clin. 2014; 32:21-32,

5. Tai C, Gan T, Zou L, et al. Effect of angiotensin-converting enzyme inhibitors and angiotensin II receptor blockers on cardiovascular events in patients with heart failure: a meta-analysis of randomized controlled trials. BMC Cardiovasc Disord. 2017;17:257.

6. Khan MS, Fonarow GC, Ahmed A, et al. Dose of Angiotensin-Converting Enzyme Inhibitors and Angiotensin Receptor Blockers and Outcomes in Heart Failure: A Meta-Analysis. Circ Heart Fail. 2017;10:e003956.

7. Heran, Balraj S, Bassett K, et al. Angiotensin receptor blockers for heart failure. The Cochrane database of systematic reviews vol. 2012,4 CD003040. 18 Apr. 2012, doi:10.1002/14651858.CD003040.pub2

8. Gronda E, Bologna A, Mangiavacchi M, et al. Il trattamento con betabloccanti nello scompenso cardiaco avanzato [Treatment with beta blockers in advanced heart failure]. Italian heart journal. Supplement : official journal of the Italian Federation of Cardiology 2000; 1, 1011-18.

9. Kotecha D, Holmes J, Krum H, et al; Beta-Blockers in Heart Failure Collaborative Group. Efficacy of $\beta$ blockers in patients with heart failure plus atrial fibrillation: an individual-patient data meta-analysis. Lancet. 2014; 384:2235-43

10. Swedberg K, Komajda M, Böhm M, et al. Ivabradine and outcomes in chronic heart failure (SHIFT): a randomised placebo-controlled study. Lancet 2010; 376:875-85

11. Digitalis Investigation Group. "The effect of digoxin on mortality and morbidity in patients with heart failure." The New England journal of medicine vol. 336, 8:525-33.

12. Young JB, Gheorghiade M, Uretsky BF, et al. Superiority of "triple" drug therapy in heart failure: insights from the PROVED and RADIANCE trials. Prospective
Randomized Study of Ventricular Function and Efficacy of Digoxin. Randomized Assessment of Digoxin and Inhibitors of Angiotensin-Converting Enzyme. J Am Coll Cardiol. 1998; 32:686-92

13. Struthers, Allan D. "The clinical implications of aldosterone escape in congestive heart failure." European Journal of heart failure 2004; 6:539-45.

14. Barr C, Lang C, Hanson J et al. Effects of adding spironolactone to an angiotensin-converting enzyme inhibitor in chronic congestive heart failure secondary to coronary artery disease Am J Cardio 1995; 76:1259-65

15. Pitt B, RemmeW, Zannad F, et al; Eplerenone PostAcute Myocardial Infarction Heart Failure Efficacy and Survival Study Investigators. Eplerenone, a selective aldosterone blocker, in patients with left ventricular dysfunction after myocardial infarction. N Engl J Med. 2003; 348: 1309-1321.

16. McMurray JJV, Packer M, Desai AS, et al; PARADIGM-HF Investigators and Committees. Angiotensinneprilysin inhibition versus enalapril in heart failure. $\mathrm{N}$ Engl J Med. 2014; 371:993-1004.

17. Velazquez EJ, Morrow DA, DeVore AD, et al; PIONEER-HF Investigators. Angiotensin-neprilysin inhibition in acute decompensated heart failure. N Engl J Med. 2019;380:539-48

18. Solomon SD, McMurray JJV, Anand IS, et al; PARAGON-HF Investigators and Committees. Angiotensinneprilysin inhibition in heart failure with preserved ejection fraction. N Engl J Med. 2019; 381:1609-20.

19. Pellicori P, Carubelli V. Terapia diuretica nell'insufficienza cardiaca cronica: evidenze, esperienze, prospettive [Diuretic treatment in patients with chronic heart failure: evidences, experiences, and current perspectives]. G Ital Cardiol 2017; 18:129-138.

20. Bugger H, Abel ED. Molecular mechanisms of diabetic cardiomyopathy. Diabetologia. 2014; 57:660-71.

21. Fitchett D, Inzucchi SE, Cannon CP, et al. Empagliflozin Reduced Mortality and Hospitalization for Heart Failure Across the Spectrum of Cardiovascular Risk in the EMPA-REG OUTCOME Trial. Circulation. 2019; 139:1384-95.

22. Mahaffey KW, Neal B, Perkovic V, et al. Canagliflozin for Primary and Secondary Prevention of Cardiovascular Events: Results From the CANVAS Program (Canagliflozin Cardiovascular Assessment Study). Circulation. 2018; 137:323-34.

23. Petrie MC, Verma S, Docherty KF, et al. Effect of dapagliflozin on worsening heart failure and cardiovascular death in patients with heart failure with and without diabetes. JAMA. 2020; 323:1353-68.

24. Packer M, Anker SD, Butler J, et al. Cardiovascular and Renal Outcomes with Empagliflozin in Heart Failure. N Engl J Med. 2020;383:1413-24.

25. Taylor AL, Ziesche S, Yancy C, et al; African-American Heart Failure Trial Investigators. Combination of isosorbide dinitrate and hydralazine in blacks with heart failure. N Engl J Med. 2004;351:2049-57.

26. Armstrong PW, Roessig L, Patel MJ, et al. A Multicenter, Randomized, Double-Blind, Placebo-Controlled Trial of the Efficacy and Safety of the Oral Soluble Guanylate Cyclase Stimulator: The VICTORIA Trial. JACC Heart Fail. 2018; 6:96-104.

27. Teerlink JR, Diaz R, Felker GM, et al Omecamtiv Mer- 
cabil in Chronic Heart Failure With Reduced Ejection Fraction Rationale e Design of GALACTIC-HF JACC Heart Failure 2020; 8: 329-40

28. Teerlink JR, Diaz R, Felker GM, et al Cardiac Myosin Activation with Omecamtiv Mercabil in Systolic Heart Failure, New Engl J Medicine November 13, 2020. DOI: 10.1056/NEJMoa2025797

29. Ponikowski P, Voors A, Anker S, et al. 2016 ESC Guidelines for the diagnosis and treatment of acute and chronic heart failure. European Heart Journal 2016; 37 : 2129-200

30. Priori SG, Blomstrom-Lundqvist C, et al.. 2015 ESC Guidelines for the management of patients with ventricular arrhythmias and the prevention of sudden cardiac death: the Task Force for the Management of Patients with Ventricular Arrhythmias and the Prevention of Sudden Cardiac Death of the Europe. Eur Heart J 2015;36:2793-867.

31. Sweeney MO, Hellkamp AS, Ellenbogen KA, et al.Adverse effect of ventricular pacing on heart failure and atrialfibrillation among patients with normal baseline QRS duration in a clinical trial of pacemaker therapy for sinus node dysfunction.Circulation 2003;107:2932-7.

32. O'keefe JH, Jones PG, Thompson RC, et al. Effect of chronic right ventricular apical pacing on left ventricular function. Am J Cardiol 2005;95:771-3

33. Shimony A, Eisenberg MJ, Filion KB, et al. Beneficial effects of right ventricular non-apical vs. apical pacing: a systematic review and meta-analysis of randomizedcontrolled trials.Europace 2012;14:81-91

34. Cleland JGF, Daubert J-C, Erdmann E, Freemantle N, Gras D,Kappenberger L, et al. The effect of cardiac resynchronization on morbidity and mortality in heart failure. N Engl J Med. 2005;352:1539-49.

35. Curtis AB, Worley SJ, Adamson FB, et al. Biventricular pacing for atrioventricular block and systolic dysfunction for the biventricular versus right ventricular pacing in heart failure patients with atrioventricular block (Block HF) Trial Investigators. N Engl J Med 2013; 368: 1585-93

36. Cleland JG, Abraham WT, Linde C, et al. An individual patient meta-analysis of five random-ized trials assessing the effects of cardiac resynchronization therapy on morbidity and mortality in patients with symptomatic heart fail-ure. Eur Heart J. 2013; 34:3547-56.

37. Jones S, Lumens J, Sohaib SMA, et al. Cardiac resynchronization therapy: mechanisms ofaction and scope for further improvement in cardiac function. Europace. 2017;19:1178-86.

38. Ahran D. Arnold, Matthew J. Shun-Shin, Daniel Keene, et al. His Resynchronization Versus Biventricular Pacing in Patients with Heart Failure and Left Bundle Branch Block. J Am Coll Cardiol 2018; 72: 3112-22.

39. Upadhyay GA, Vijayaraman P, Nayak HM, et al. Ontreatment comparison between corrective His bundle pacing and biventricular pacing for cardiac resynchronization: a secondary analysis of His-SYNC. Heart Rhythm. 2019;16(12):1797-1807.

40. Zhang W, Huang J, Qi Y, et al. Cardiac resynchronization therapy by left bundle branch area pacing in patients with heart failure and left bundle branch block. Heart Rhythm. 2019;16:1783-90

41. Occhetta E., Dell'Era G, Sartori C, et al: Right bundle branch block and heart failure: Can a bifocal right ventricular pacing be an alternative to biventricular pacing? Cor et Vasa 2016; 4:391-e395

42. Coluccia G, Vitale E, Corallo S, et al. Additional benefits of nonconventional modalities of cardiac resynchronization therapy using His bundle pacing. J Cardiovasc Electrophysiol 2020;31:647-57

43. Nagele, H .; S. Behrens; C. Eisermann et al. Cardiac contractility modulation in non responders to cardiac resynchronization therahy. Europace 2008; 10: 1375-80

44. Markus Zabel, Rik Willems, Andrzej Lubinski, et al. The EU-CERT-ICD StudyInvestigators. Clinical effectiveness of primary preventionimplantable cardioverterdefibrillators: results of the EU-CERT-ICD controlled multicentrecohort study. European Heart Journal 2020; 41, 3437-47

45. Raphael CE, Finegold JA, Barron AJ, et al. The effect of duration of follow-up and presence of com-peting risk on lifespan-gain from implantable cardioverter defibrillator therapy: who benefits the most? Eur Heart J 2015;36:1676-88

46. Zannad F, Briancon S, Juilliere Y et al. And the EPICAL Investigators. Incidence, clinical and etiologic features and outcome of advanced chronic heart failure: the EPICAL syudy. J Am Coll Cardiol 1999; 33:734-42

47. O'Connor CM, Gattis WA, Uretsky BF et al. Continuous intravenouos dobutamine in associated with an increased risk of death in patients with advanced heart failure: insights from Flolan International Randomized Survival Trial (FIRST). Am Heart J 1999; 138:78-86

48. Birati Edo Y and Jessup Marriell. Left ventricular assist devices in the management of heart failure. Cardiac Failure Review 2015; 1:25-30

49. Kirklin JK, Naftel DC, Stevenson LW et al. INTERMACS database for durable device for circulatory support: first annual report. J Heart Lung Transplant 2008; 27:1065-72

50. Stevenson LW, Pagani FD, Young JB et al. INTERMACS profiles of advanced heart failure: the current practice. J Heart Lung Transplant 2009; 28:535-41

51. Kirklin JK, Naftel DC, Kormos RL et al. Second INTERMACS annual report: more than 1000 primary left ventricular assist device implants. J Heart Lung Transplat 2010;29:1-10

52. Kirklin JK, Naftel DC, Pagani FD et al. Sixth INTERMACS annual report: a 10,000-patient database. J heart Lung Transplant 2014; 33: 555-64

53. Chen YS, Yu HJ, Huang SC et al. Experience and results of extracorporeal membrane oxygenation in treating fulminant myocarditis with shock: what mechanical support should be considered first? J heart Lung Transplant $2005 ; 24: 81-7$

54. Meyns B, Stolinski J Leunens V et al. Left ventricular support by catheter mounted axial flow pump reduces infart size. J Am Coll Cardiol 2003; 41: 1087-95

55. Rose EA, Gelijns AC, Moskowitz AJ, et al. for the Randomized Evaluation of Mechanical Assistence for the treatment of Congestive Haert Failure (REMATCH) Study Group. Long term use of a left ventricular assist device for end stage heart failure. N Eng J Med 2001; 345: 1435-43

56. Hetzer R, Muller J, Weng Y et al. Cardiac recovery in dilated cardiomyopathy by unloading with a left ventricular assist device. Ann Thorac Surg 1999; 68:742-9. 


\title{
Opportunità terapeutiche nel paziente con scompenso cardiaco diastolico
}

\author{
Aderville Cabassi, ${ }^{1}$ Giuseppe Regolisti ${ }^{2}$ \\ ${ }^{1}$ Centro Ipertensione Arteriosa e Studio Malattie Cardiorenali, S.S. Fisiopatologia Medica, Clinica Medica Generale e Terapia \\ Medica; e ${ }^{2}$ Clinica e Immunologia Medica, Azienda Ospedaliera-Universitaria di Parma, Università di Parma, Parma, Italia
}

\section{Introduzione}

\section{Cenni epidemiologici e clinici}

La sindrome clinica di insufficienza cardiaca con frazione di eiezione conservata (HFpEF) si caratterizza per sintomi e segni di scompenso cardiaco ma con frazione di eiezione ventricolare sinistra normale o quasi normale $(\geq 50 \%)$. Veniva in passato definita come scompenso cardiaco diastolico, e in effetti la maggior parte dei pazienti con HFpEF presenta volumi ventricolari sinistri normali e chiare evidenze di disfunzione diastolica. La HFpEF si sviluppa dall'interazione di diversi fattori di rischio che causano disfunzione d'organo. Questi fattori di rischio e la presenza di comorbidità condiziona fortemente le modificazioni strutturali e funzionali che portano al quadro clinico di HFpEF, anche se i meccanismi cellulari e fisiopatologici specifici sono tuttora oggetto di approfonditi studi e non ancora del tutto chiariti. Cosi come nella insufficienza cardiaca a frazione di eiezione ridotta (HFrEF) alcune condizioni di comorbidità tendono ad essere più comuni (malattia ischemica coronarica), nell'HFpEF l'ipertensione arteriosa e l'obesità risultano essere più prevalenti. In generale il numero di condizioni di comorbidità è più alto nel soggetto con HFpEF senza tuttavia determinare un'unica e chiara discrimina fra le due forme di scompenso. ${ }^{1}$ La prevalenza di HFpEF nel nostro

Corrispondente: Aderville Cabassi, Centro Ipertensione Arteriosa e Studio Malattie Cardiorenali, Unità di Fisiopatologia Medica, Clinica e Terapia Medica, Dipartimento di Medicina e Chirurgia, Università di Parma, via Gramsci 14, 43126 Parma, Italy.

Tel.: +39.0521033184 - Fax: +39.0521033185

E-mail: aderville.cabassi@unipr.it

Articolo pubblicato secondo la Creative Commons Attribution NonCommercial 4.0 License (CC BY-NC 4.0).

${ }^{\circ}$ Copyright: the Author(s), 2021

Licensee PAGEPress, Italy

QUADERNI - Italian Journal of Medicine 2021; 9(2):e5 continente raggiunge il 5\% della popolazione con più di 60 anni d'età e tale percentuale, che costituisce tra il 50 e il $60 \%$ delle diagnosi di insufficienza cardiaca, è destinata ulteriormente ad incrementare nelle prossime due decadi in relazione all'invecchiamento, alla maggior prevalenza di patologie quali l'ipertensione arteriosa, l'obesità, il diabete mellito e la broncopneumopatia cronica ostruttiva..$^{2-5} \mathrm{La} \mathrm{HFpEF}$ costituisce già oltre la metà delle ospedalizzazioni per insufficienza cardiaca. ${ }^{2,6}$ L'incidenza di HFpEF risulta variare tra il 37 e il $53 \%$ in alcuni studi che hanno considerato un lungo follow-up di soggetti che non presentavano insufficienza cardiaca (Cardiovascular Health Study, Framingham Heart Study e Prevention of Renal and Vascular End-Stage Disease Study); un ulteriore incremento dell'incidenza si è osservato in un campione di popolazione più anziana. ${ }^{7} \mathrm{Da}$ questi studi epidemiologici emerge come l'invecchiamento e il sesso femminile risultano più frequenti nell'HFpEF rispetto a quello a frazione d'eiezione ridotta (HFrEF). I riscontri in letteratura da studi di coorte evidenziano un'età media più elevata di almeno 6 anni nel gruppo affetto da HFpEF rispetto a coloro con $\mathrm{HFrEF}^{8}$ e che le donne sono circa oltre un terzo più prevalenti nel primo gruppo rispetto al secondo. ${ }^{9}$ Sulle differenze etniche ci sono elementi a favore di una maggior prevalenza e incidenza nella popolazione nera, soprattutto nelle donne, con il rischio di ospedalizzazione che risulta almeno il doppio rispetto alla popolazione caucasica. ${ }^{10,11}$ Come abbiamo accennato prima e in relazione alla elevata prevalenza di vari fattori di rischio cardiovascolare e comorbidità nel paziente affetto da $\mathrm{HFpEF}$, in particolare della ipertensione arteriosa, nel corso degli ultimi tre decenni questa condizione è stata nel tempo considerata la classica evoluzione della cardiopatia ipertensiva. La malattia ischemica coronarica, molto più frequente nei pazienti con $\mathrm{HFrEF}$, è comunque presente anche nei soggetti con $\mathrm{HFpEF}$ (fra $1 / 3$ e la metà dei pazienti in relazione agli studi). ${ }^{12} \mathrm{La}$ presenza di malattia coronarica comporta, come d'altra parte atteso nei pazienti con HFpEF, un rischio di maggior mortalità. ${ }^{13}$ Altre comorbidità devono essere considerate molto prevalenti nei pazienti affetti da 
HFpEF, e contribuiscono alle modificazioni cardiache morfostrutturali e funzionali. Queste includono l'obesità, che è presente in oltre la metà dei soggetti, il diabete mellito (fra il 20 e il 40\%), l'insufficienza renale (fino ad un terzo dei pazienti) e la fibrillazione atriale. ${ }^{12}$ L'obesità, nello specifico, risulta un elemento importante e molto prevalente nei soggetti con HFpEF (arrivando ad oltre i tre quarti dei pazienti) $;{ }^{14} \mathrm{i}$ livelli circolanti di peptidi natriuretici nel paziente obeso risultano inferiori rispetto a soggetti normopeso, in quanto le cellule adipose posseggono elevata espressione di recettori di clearance dei peptide natriuretici con conseguente riduzione della loro concentrazione plasmatica. ${ }^{15}$

Tali comorbidità, in particolare ipertensione arteriosa e diabete mellito, non discriminano fra HFpEF e HFrEF essendo chiaramente questi tutti fattori per lo sviluppo della malattia ischemica micro e macrovascolare coronarica.

L'associazione tra insufficienza cardiaca e diabete mellito è evidente da dati ormai consolidati in letteratura che indicano come i pazienti diabetici hanno un rischio almeno raddoppiato di sviluppare insufficienza cardiaca nel corso della loro vita; tale rischio arriva anche a 5 volte in alcune popolazioni, mentre circa il $45 \%$ delle persone con insufficienza cardiaca ha un diabete sottostante. ${ }^{16,17}$ Tale associazione è particolarmente robusta con $\mathrm{HFpEF}$ rispetto a HFrEF.

I soggetti affetti da HFpEF presentano un aumentato rischio di mortalità per tutte le cause e di mortalità cardiovascolare rispetto alla popolazione sana ${ }^{18}$ Diversi studi hanno evidenziato tassi di mortalità sovrapponibile fra soggetti con HFpEF e coloro con $\mathrm{HFrEF},{ }^{19,20}$ tuttavia questo non risulta un dato univoco in quanto altre evidenze dalla letteratura hanno mostrano una differenza fra HFpEF nei confronti di $\mathrm{HFrEF}$ con tassi minori nei primi rispetto ai secondi. ${ }^{18,21}$ La mortalità ospedaliera che si verifica durante un ricovero per HF è attorno al $4 \%$ e la mortalità a 30 giorni dopo la dimissione è attorno al 10\%. I tassi di ospedalizzazione, la durata del ricovero e la qualità della vita risultano sovrapponibili tra HFpEF rispetto a HFrEF. ${ }^{22,23}$ L'incidenza di mortalità cardiovascolare è minore negli HFpEF rispetto a HFrEF mentre quella non cardiovascolare risulta più alta. ${ }^{8}$

I pazienti con HFpEF hanno minor tolleranza allo sforzo, affaticamento e dispnea come tipicamente si osserva nel paziente con scompenso cardiaco; tale situazione clinica progredisce con peggioramento dei sintomi con livelli di attività progressivamente minori fino al quadro di congestione venosa, che determina il ricovero ospedaliero. Il quadro clinico dei soggetti affetti da HFpEF è in gran parte correlato ad anomalie emodinamiche che dipendono da alterazioni strutturali e funzionali cardiache..$^{24,25}$ In aggiunta alle alterazioni cardiache, risultano molto importanti le anomalie del sistema vascolare periferico e polmonare associate a stress ossidativo e a disfunzione endoteliale, del metabolismo del tessuto muscolare scheletrico e adiposo. ${ }^{26-30} \mathrm{Ci}$ sono una serie di difficoltà alla comprensione dei meccanismi fisiopatologici alla base della alterazioni cardiache e sistemiche dei pazienti con HFpEF. Una prima difficoltà nasce dal fatto che non ci sono modelli sperimentali consolidati di HFpEF, sebbene siano disponibili modelli che riassumono alcune caratteristiche della forma di insufficienza cardiaca che si osserva nell'uomo (accelerato invecchiamento, obesità, ipertensione arteriosa, attivazione neurormonale). ${ }^{31-35}$ Tuttavia risulterà fondamentale la verifica sui tessuti umani della possibile traslazione dei risultati ottenuti dai modelli sperimentali.

\section{Opzioni terapeutiche nello scompenso diastolico}

La terapia della condizione di scompenso diastolico o HFpEF è per grande parte determinata dalle condizioni cliniche e dai fattori di rischio associati e dai sintomi, poiché prove dirette che indichino chiaramente opzioni terapeutiche farmacologiche chiare risultano ancora limitate. I diuretici sono usati per trattare il sovraccarico di volume, ma occorre prestare attenzione alla eccessiva deplezione volemica e alla eccessiva riduzione del precarico. Il paziente che ha disfunzione diastolica con una piccola camera ventricolare sinistra rigida risulta suscettibile a un'eccessiva riduzione del precarico e ad una eventuale ipotensione arteriosa. Altre classi di farmaci come quelli che interferiscono con il Sistema renina-angiotensina-aldosterone, i calcio-antagonisti, i $\beta$-bloccanti sono raccomandati per trattare l'ipertensione arteriosa ma mancano evidenze dirette di comprovata efficacia per modificare gli esiti clinici nei pazienti con HFpEF. Non sono raccomandati nei pazienti con HFpEF i farmaci quali gli inibitori delle fosfodiesterasi-5, i nitrati organici e la digossina (se si esclude il suo utilizzo per il controllo della risposta ventricolare nella fibrillazione atriale).

Pertanto l'appropriata gestione delle condizioni cliniche associate e dei fattori ri rischio presenti risulta necessaria per poter influenzare la storia naturale di questa forma di scompenso. Queste condizioni includono come già indicato sopra, la ipertensione arteriosa, la obesità, il diabete mellito, le malattie polmonari associate, la malattia coronarica, la fibrillazione atriale, la anemia, la insufficienza renale cronica e le alterazioni del sonno legate ad apnee ostruttive.

La terapia antipertensiva ha dimostrato di ridurre l'incidenza di scompenso cardiaco. Anche la terapia ipolipemizzante, che risulta raccomandata per la prevenzione primaria e secondaria delle malattie 
cardiovascolari, potrebbe essere benefica nei soggetti con HFpEF secondo dati osservazionali, la cui conferma necessità di trials clinici di intervento dedicati. ${ }^{36} \mathrm{Il}$ trattamento dell'obesità nei pazienti con HFpEF rappresenta un elemento importante; gli HFpEF obesi risultano meno efficienti in termini di capacità d'esercizio e con maggior espansione volemica rispetto agli HFpEF non obesi e la restrizione calorica ha comportato un miglioramento dei parametri sopra indicati. ${ }^{37}$ Circa 10 anni orsono si è dimostrato come la qualità di vita e la capacità di esercizio dei pazienti con HFpEF risultino migliorati da programmi di allenamento fisico. ${ }^{38} \mathrm{Da}$ quel momento numerosi studi hanno confermato questi vantaggi indicando sia effetti favorevoli periferici che centrali cardiaci, indicando inoltre anche effetti additivi da parte dell'esercizio fisico e della perdita di peso in soggetti HFpEF obesi. ${ }^{37}$

La fibrillazione atriale è un'aritmia comune nei pazienti affetti da HFpEF e la sua prevalenza risulta essere elevata superando oltre la metà dei soggetti. ${ }^{39} \mathrm{Il}$ ripristino e il mantenimento del ritmo sinusale rappresentano un grande vantaggio nei soggetti con HFpEF. Quando il ritmo sinusale non può ad essere conservato allora il controllo della frequenza cardiaca diviene rilevante. $\beta$-bloccanti e bloccanti dei canali del calcio sono i soliti agenti di prima linea, con la digossina utilizzata soltanto nel controllo della risposta ventricolare. Il controllo della frequenza cardiaca deve essere adeguato non soltanto a riposo ma anche in corso di esercizio lieve e moderato. La presenza di ischemia miocardica nel paziente affetto da HFpEF può dipendere da una disfunzione del microcircolo coronarico, da uno stress di parete elevato o da malattia dei vasi epicardici. ${ }^{40}$ I pazienti con HFpEF sintomatici per ischemia beneficano della terapia standard $\operatorname{con} \beta$ - bloccanti e calcio-antagonisti. La terapia con nitrati è utilizzata pur con attenzione in quanto l'eccessiva riduzione del precarico porta ad ipotensione. La rivascolarizzazione coronarica nei vasi resistente alla terapia farmacologica si è mostrata utile in termini di sopravvivenza. ${ }^{41}$

\section{Evidenze da trials clinici in pazienti con HFpEF}

Come è ben noto, dalla pubblicazione del primo trial di intervento su pazienti con HFrEF circa 35 anni orsono le evidenze si sono moltiplicate e hanno dimostrato anche di recente in questo gruppo di pazienti i grandi benefici di varie classi farmacologiche e quindi le diversificate opzioni terapeutiche (Tabella 1). Dal primo studio di intervento sul gruppo di pazienti affetti da HFpEF sono passati circa 18 anni e le opzioni terapeutiche cosi ampie per coloro che hanno HPrEF non si sono confermate (Figura 1). I risultati dei trials clinici nei pazienti con $\mathrm{HFpEF}$ hanno dato risultati neutri fino ad oggi e stando anche alle indicazione delle linee guida europee ed americane la maggior parte della gestione per ora appare diretta verso le condizioni cliniche e i fattori di rischio associati a tale forma di scompenso cardiaco, o al trattamento dei sintomi. Le raccomandazioni delle due Società Scientifiche fanno riferimento al buon controllo dell'ipertensione arteriosa sistolica e diastolica e all'uso dei diuretici per gestire e migliorare i sintomi legati alla congestione. ${ }^{42,43}$ I risultati degli studi clinici di intervento farmacologico o di risincronizzazione cardiaca efficaci nei pazienti con HFrEF, non hanno impattato sulla morbilità e la mortalità in $\mathrm{HFpEF}$, suggerendo differenze importanti nella fisiopatologia fra le due forme di scompenso.

Gli inibitori del sistema renina-angiotensina-

Tabella 1. Effetti di farmaci nello scompenso sistolico (HFrEF) e diastolico (HFpEF).

\begin{tabular}{|c|c|c|c|}
\hline Trattamento & HFrEF & HFpEF & Studi Clinici HFpEF \\
\hline B-bloccanti & SI & $?$ & Seniors, Elandd, J-DHF \\
\hline ACE-i/ARB & SI & $\mathrm{NO}$ & Charm, I-Preserve, PEP-CHF \\
\hline Digitale & $?$ & NO & Dig-PEF \\
\hline Inibitori PDE-5 & & $\mathrm{NO}$ & Relax-HF \\
\hline Mineralcorticoidi & SI & $\mathrm{NO} / \mathrm{SI}$ & Topcat \\
\hline Idralazina/nitrati & SI & NO & Neat-HFpEF \\
\hline Antagonisti endotelina & NO & SI p II & Sitaxesentan \\
\hline Sacubitril/valsartan & SI & SI $p$ II & Paramount/Paragon \\
\hline CRT & SI & - & Karolinska-Rennes (KaRen) \\
\hline ICD & SI & - & \\
\hline Esercizio Fisico & SI & $+/-$ & \\
\hline CardioMEMS & SI & SI & Champion \\
\hline
\end{tabular}


aldosterone (inibitori dell'enzima di conversione, antagonisti dei recettori AT1 dell'angiotensina II e antialdosteronici), unitamente ai $\beta$-bloccanti adrenergici selettivi e non, e agli ARNI (farmaci che combinano un inibitore della neprilisina e un AT1-bloccante), bloccando la attivazione neurormonale e potenziando l'azione dei peptide natriuretici si sono dimostrati efficaci nel ridurre morbilità e mortalità in HFrEF e fanno parte delle opzioni terapeutiche fondanti la terapia di questa condizione clinica (Tabella 1).

Nell'HFpEF ci sono alcuni grandi trials che hanno verificato sicurezza ed efficacia di varie classi di farmaci interferenti con il sistema renina-angiotensinaaldosterone già testate nelle forme di HFrEF.

Nello specifico, il trial CHARM-Preserved (Candesartan in Heart Failure-Preserved - CHARMPreserved) reclutando pazienti (classe NYHA II-IV) con LVEF $>40 \%$ ha dimostrato dopo un follow-up mediano di oltre 3 anni come il candesartan non determinava un vantaggio sulla morte cardiovascolare combinata a ospedalizzazione rispetto al placebo. ${ }^{44}$ Scorporando l'end point primario nei suoi 2 elementi si osservava che il gruppo in terapia con farmaco attivo non impattava sulla morte cardiovascolare, ma è stata osservata una tendenza ad una riduzione della ospedalizzazione che ha portato le linee Guida Americane a indicare una classe di raccomandazione IIb per la diminuzione della riospedalizzazione. ${ }^{43}$ Tale rilievo relativo alla diminuzione della ospedalizzazione con un antagonista dei recettori AT1 dell'angiotensina II non si è confermato con l'irbesartan nello studio I-preserve (Irbesartan in Heart Failure With Preserved Systolic Function), ${ }^{45}$ né con un inibitore dell'enzima di conversion perindopril nella popolazione anziana (PEP-CHF, Perindopril in Elderly People with Chronic Heart Failure). ${ }^{46} \mathrm{Un}$ discorso a parte meritano i risultati dello studio TOPCAT (Treatment of Preserved Cardiac Function Heart Failure With an Aldosterone Antagonist) che ha mostrato come lo spironolattone comportasse un beneficio sulle componenti combinate e separate di morte cardiovascolare, ospedalizzazione per scompenso $\mathrm{o}$ arresto cardiaco abortito nella popolazione reclutata nel continente americano ma non nell'est dell'Europa ${ }^{47}$ a fronte però di un incremento di creatininemia e della potassiemia (risultata raddoppiata) nel gruppo del farmaco attivo. Tale osservazione non si era rilevata quando l'intera popolazione del trial reclutata veniva analizzata comprendendo coloro che erano arruolati nelle Americhe, in Russia e in Georgia (hazard ratio 0,89, CI 95\% 0,77-1,04). ${ }^{48}$ Nello studio che ha arruolato 3445 pazienti con $L V E F \geq 45 \%$ e che sono stati seguiti per un periodo di 3,3 anni di follow-up medio si sono riscontrate una riduzione delle ospedalizzazioni per scompenso cardiaco nel gruppo trattato con spironolattone $(12,0 \%)$ rispetto al gruppo placebo $(14,2 \%$, hazard ratio, $0,83,95 \%$ CI $0,69-0,99)$. Per spiegare le differenze fra i sottogruppi arruolati nelle Americhe e in Europa dell'Est (Russia e Georgia) sono state dimostrate bassissime percentuali di aderenza e persistenza in terapia spironolattone nel secondo sottogruppo. Al pari del candesartan nelle linee guida americane $^{43}$ anche la terapia con spironolattone ha ottenuto una classe di raccomandazione IIb. La raccomandazione dell'uso di spironolattone risulta più debole rispetto a quanto indicato nei pazienti con HFrEF; pertanto l'uso degli antialdosteronici deve essere più attento utilizzando valori di potassiemia più bassi per l'inizio del trattamento $(\leq 4,7 \mathrm{mEq} / \mathrm{L}$ e il filtrato glomerulare stimato eGFR $\geq 30 \mathrm{~mL} / \mathrm{min}$ per $1,73 \mathrm{~m}^{2}$ ). Se la

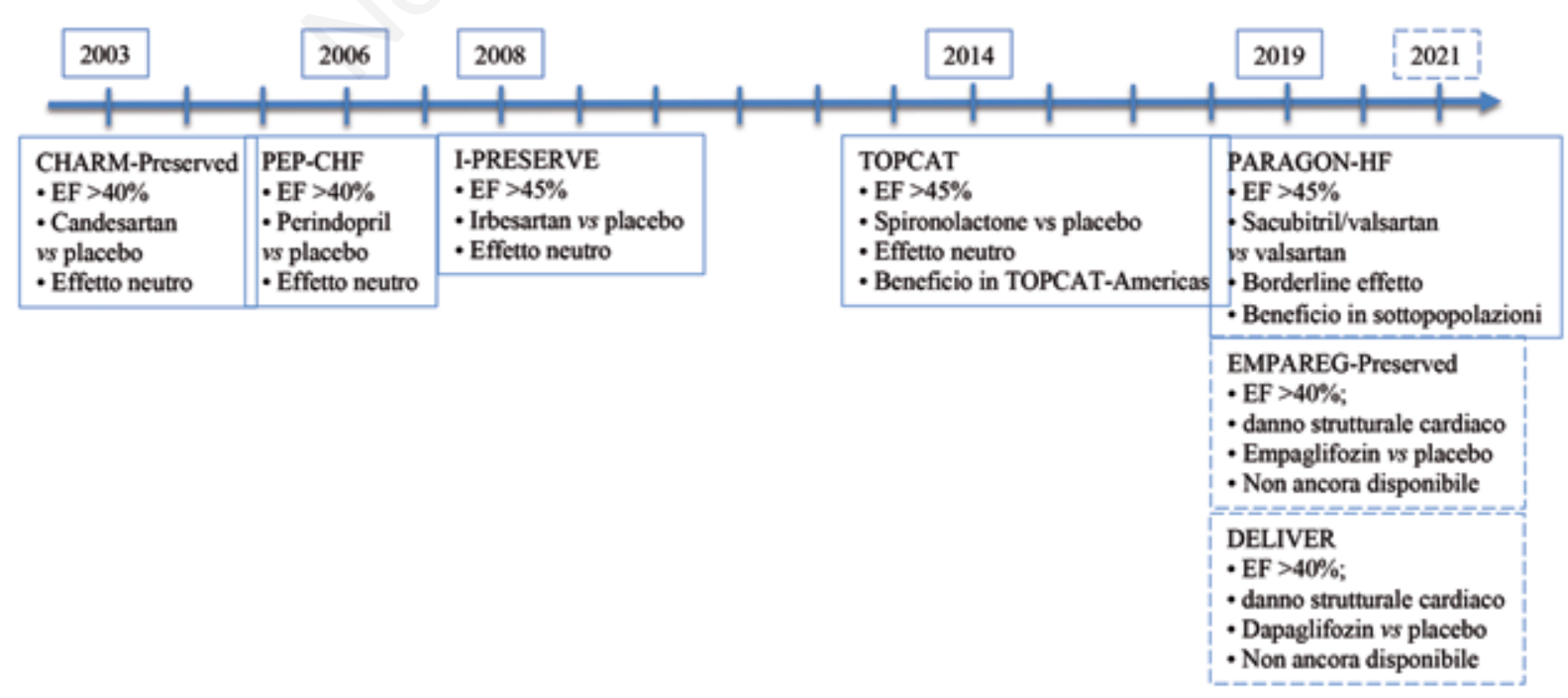

Figura 1. La cronologia degli studi HFpEF e l'effetto sugli endpoint primari. 
potassiemia supera $5,5 \mathrm{mEq} / \mathrm{L}$, il farmaco antialdosteronico deve essere ridotto o sospeso valutando quali cause possono averla determinata (concomitante uso di alte dosi di antagonisti AT1 dell'angiotensina II, ACE inibitori). L'effetto dello spironolattone sui valori di pressione arteriosa sistolica nei pazienti con $\mathrm{HFpEF}$ è modesto (attorno a $3 \mathrm{mmHg}$ nello studio TOPCAT). Qualora con l'inizio del trattamento con anti-aldosteronici si verificassero riduzioni significative della pressione arteriosa occorre ridurre le dosi di altri antipertensivi concomitanti e solo successivamente ridurre lo spironolattone.

$\mathrm{Ci}$ sono inoltre evidenze che hanno mostrato come nella patogenesi della forma di HFpEF le elevate pressioni di riempimento cardiaco giocano un ruolo nella progressione del quadro clinico e pertanto la loro riduzione impatta positivamente sulla prognosi. Nello studio CHAMPION (CardioMEMS Heart Sensor Allows Monitoring of Pressure to Improve Outcomes in NYHA Class III Heart Failure Patients) la conoscenza dei parametri di emodinamica centrale con CardioMEMS ha ridotto in maniera significativa la ospedalizzazione dei pazienti rispetto a coloro non monitorati e trattati in maniera standard. ${ }^{49}$ Tale risultato può dipendere da una migliore titolazione dei farmaci, in particolare dei diuretici che consentirebbero una miglior gestione dei volumi.

Un'altra classe di farmaci (ARNI) si è resa disponibile, la cui unica molecola commercializzata è rappresentata da Sacubitril/valsartan (precedentemente noto come LCZ696) che associa un inibitore della neprilisina all'antagonista del recettore AT 1 dell'angiotensina II. Tale combinazione di effetti consente la contemporanea disattivazione del sistema renina-angiotensina-aldosterone attraverso il blocco dei recettori dell'angiotensina II di tipo 1 e incrementa la concentrazione dei peptide natriuretici (ANP, BNP) attraverso l'inibizione della neprilisina, l'enzima responsabile della loro degradazione. A tal proposito, nel 2019 è stato pubblicato un altro importante trial, il PARAGON-HF, che ha valutato su un'ampia popolazione di pazienti con HFpEF (Prospective Comparison of Angiotensin Receptor Neprilysin Inhibitor With Angiotensin Receptor Blocker Global Outcomes in $\mathrm{HFp} E \mathrm{~F}$ ), con scompenso classe NYHA II$\mathrm{IV}, \mathrm{EF} \geq 45 \%$, evidenza di rimodellamento strutturale cardiaco, aumento dello spessore della parete ventricolare sinistra o ingrandimento dell'atrio sinistro, almeno un precedente ricovero per scompenso cardiaco, o elevato livello di peptidi natriuretici, l'efficacia di sacubitril-valsartan rispetto a valsartan da solo su un outcome primario che si componeva di ospedalizzazioni per insufficienza cardiaca (sia prima che ricorrente) e morte per cause cardiovascolari. ${ }^{50} \mathrm{Gli}$ end-point secondary includevano il cambio di classe NYHA, un peggioramento della funzione renale (definito come una diminuzione del filtrato glomerulare del $50 \%$ o superiore), il miglioramento della qualità di vita (indicato attraverso i punteggi del questionario sulla cardiomiopatia di Kansas City, KCCQ), morte per qualsiasi causa. Si sono osservati 894 eventi primari in 526 pazienti nel gruppo sacubitril-valsartan e 1009 eventi primari in 557 pazienti nel gruppo valsartan (relative ratio, 0,87; CI 95\%, 0,75-1,01; $\mathrm{P}=0,06$ ). Analizzando le singole componenti dell'end point primario, non si sono evidenziate differenze per la mortalità cardiovascolare ma un $15 \%$ di riduzione di ospedalizzazioni (relative ratio 0,85 ; $95 \% \mathrm{CI}, 0,72$ $1,00)$. Anche la classe NYHA è risultata migliorata significativamente nel gruppo sacubitril-valsartan con però una maggiore incidenza di ipotensione $\mathrm{e}$ angioedema e una minore incidenza di iperkaliemia. Un elemento importante da sottolineare è rappresentato dal fatto che analizzando $i$ sottogruppi (già prespecificati nel disegno dello studio), è risultato che differenze si sono evidenziate con un possibile beneficio nel braccio trattato con sacubitril-valsartan nei pazienti con frazione di eiezione $\leq 57 \%$ e nel sesso femminile. Nello specifico, nelle donne si è osservato un $27 \%$ di riduzione dell'end-point primario (relative ratio, 0,73; 95\% CI, 0,59-0,9), nelle pazienti che assumevano sacubitril/valsartan, rispetto ad una differenza non significativa nel sesso maschile (relative ratio 1,03; 95\% CI, 0,85-1,25). In maniera analoga, nei pazienti con $\mathrm{LVEF} \leq$ alla mediana $(57 \%)$ si è osservato un beneficio della terapia con sacubitril/valsartan (RR, 0,78; 95\% CI, 0,64-0,95) rispetto a quelli con EF superiore (RR, 1; 95\% CI, 0,81-1,23). Lo studio PARAGON-HF estende ad una frazione di eiezione più alta l'utilizzo di una classe di farmaci nuova (ARNI); non è la prima volta che un farmaco efficace nella HFrEF (come dimostrato nello studio PARADIGM$\mathrm{HF})$, lo è anche nei pazienti con disfunzione sistolica con EF (40\%-49\%) (esempi in letteratura con spironolattone e $\beta$-bloccanti). ${ }^{51}$ L'avvento degli ARNI risulta di grande rilevanza clinica anche nella popolazione con $\mathrm{HFpEF}$ anche se è fondamentale proseguire con gli studi volti a comprendere i meccanismi alla base dello sviluppo e della progressione di questa ampia popolazione eterogenea dei soggetti con scompenso cardiaco diastolico.

In relazione a quanto già riportato sopra riguardo l'importanza delle comorbilità e dei fattori di rischio associate nello sviluppo e nella progressione dello $\mathrm{HFpEF}$, il diabete è molto frequente e condiziona in maniera importante la prognosi dei pazienti scompensati. A parte la metformina, che è stata ed è tuttora il trattamento di scelta nei pazienti con scompenso cardiaco (ma che diventa controindicata qualora si associno una compromissione grave della funzionalità epatica o renale), i glitazoni che risultano controindicati per i loro effetti sodio-ritentivi, ${ }^{42}$ di 
recente sono emersi dati robusti circa l'utilizzo delle glifozine, o meglio degli inibitori del co-trasportatore sodio-glucosio di tipo 2 (SGLT2) nei pazienti affetti da scompenso cardiaco (in particolare HFrEF). Gli inibitori di SGLT2 sono stati sviluppati come farmaci antiperglicemizzanti e includono canagliflozin, dapagliflozin, empagliflozin ed ertugliflozin ed inibiscono SGLT2 a livello del tubulo contorto prossimale del nefrone inducendo diuresi osmotica glicosurica e natriuresi. Questi farmaci comportano nei soggetti diabetici un deciso miglioramento nel controllo metabolico (glicolipemico) e del peso corporeo. ${ }^{52}$ In maniera del tutto indipendente dalle loro azioni sulla glicemia, questa nuova classe di farmaci ha dimostrato dal punto di vista sperimentale e non solo, effetti protettivi su alcuni dei meccanismi di pertinenza cardiovascolare e/o correlati a modificazioni dell'emodinamica renale che giocano un ruolo della progressione dello scompenso nei pazienti anche con HFpEF. Sono stati riportati nella popolazione diabetica tipo 2 una significativa riduzione di eventi cardiovascolari maggiori (hazard ratio [HR] $0,88,95 \%$ CI $0,82-0,94)$, di morte cardiovascolare (HR 0,83, 95\% CI 0,75-0,92) e anche per tutte le cause (HR 0,85, 95\% CI 0,79-0,92)..$^{53} \mathrm{I}$ benefici cardiovascolari osservati in questi studi non possono essere completamente spiegati da miglioramenti nei fattori di rischio come il controllo metabolico o la riduzione pressoria ma coinvolgono altri meccanismi. ${ }^{54}$ Questi meccanismi includono la capacità di ridurre il grado di infiammazione miocardica e sistemica da parte di questa classe di farmaci, di modulare la deposizione di collagene e quindi prevenire la fibrosi interstiziale cardiaca e renale. ${ }^{55-57}$ Un quadro di infiammazione sistemica a basso grado caratterizza HFpEF, e può associarsi ad infiammazione del tessuto adiposo epicardico e avventiziale il quale può trasferire alla componente tissutale miocardica, microvascolare e aortica. Si assiste ad un rilascio di citochine, ormoni (aldosterone, leptina) o all'aumentata espressione di enzimi (neprilisina) che possono contribuire alla ritenzione idrosalina, allo sviluppo di alterazioni dell'emodinamica renale fino allo stimolo alla fibrogenesi interstiziale che porta poi alla progressiva riduzione funzionale renale. ${ }^{58}$

Nelle sperimentazioni cliniche gli inibitori di SGLT2 hanno dimostrato di ridurre la quantità di tessuto adiposo epicardico in maniera indipendente dagli effetti sul peso corporeo, ${ }^{59}$ con riduzione dello stato infiammatorio adipocitario ${ }^{60}$ e renale portando ad una riduzione dell'iperfiltrazione glomerulare, una condizione che porta al danno glomerulare nel soggetto obeso e diabetico. ${ }^{61,62}$ In relazione alle importanti azioni cardiache, vascolari, renali e ai risultati degli studi clinici controllati ottenuti sui pazienti diabetici ${ }^{54,63-65}$ o su pazienti non diabetici ma con $\mathrm{HFrEF}^{66,67}$ sono stati intrapresi trials che hanno arruolato pazienti con $\mathrm{HFpEF}$, di cui avremo nei prossimi mesi risultati. Pertanto, gli inibitori di SGLT2 potrebbero rappresentare un'importante opzione terapeutica nei pazienti con HFpEF, da soli o in combinazione con antagonisti del recettore mineralocorticoide e inibitori della neprilisina.

I due studi clinici di cui siamo in attesa sono EMPEROR-Preserved e lo studio DELIVER che hanno testato empaglifozin e dapaglizofin rispettivamente. Lo studio EMPEROR-Preserved (EMPagliflozin outcomE tRial in Patients With chrOnic heaRt Failure With Preserved Ejection Fraction) è stato costruito per valutare l'effetto di empagliflozin sul rischio di morte cardiovascolare morte o ospedalizzazione per insufficienza cardiaca in pazienti con HFpEF sia diabetici che non, così come l'effetto del farmaco sul numero totale di ricoveri per insufficienza cardiaca. Questo studio ha inoltre darà informazioni circa gli effetti di empaglifozin sulla progressione del danno renale.

Il campione di pazienti arruolato (oltre 5550 soggetti) consentirà di fornire informazioni circa alcuni meccanismi patogenetici che contribuiscono allo sviluppo e alla progressione di HFpEF considerando anche l'asse che vede coinvolte la leptina, l'aldosterone e l'enzima neprilisina individuando quindi la tipologia di attivazione neurormonale il cui blocco possa risultare utile come si è già dimostrato nelle forme di scompenso HFrEF.

Allo stesso modo, lo studio DELIVER (Dapagliflozin Evaluation to Improve the LIVEs of Patients With PReserved Ejection Fraction Heart Failure), i cui risultati saranno disponibili nel corso dei prossimi mesi (completamento dello studio previsto per giugno 2021), aggiungerà informazioni fondamentali per stabilire le implicazioni terapeutiche del dapagliflozin nei pazienti HFpEF. Anche in questo caso l'end point primario è composto dal verificarsi di morte cardiovascolare, ospedalizzazione o visita urgente per insufficienza cardiaca. Tale studio si propone di confermare quanto osservato già nello studio DAPA-HF, e appunto se vi sia un beneficio in termini di incidenza di ospedalizzazione e morte cardiovascolare in chi è affetto da HFpEF simile a quello osservato nei pazienti con $\mathrm{HFrEF},{ }^{66}$ come già aveva suggerito la sottoanalisi del DECLARE-TIMI sul limitato gruppo di pazienti con $\mathrm{HFpEF}^{68}$ Entrambi gli studi clinici DELIVER and EMPEROR-Preserved, con un'ampia numerosità dei pazienti arruolati, mirano pertanto a stabilire l'impatto di un inibitore SGLT2 in pazienti con HFpEF su end points primari simili (DELIVER considera anche le visite per insufficienza cardiaca in urgenza al di fuori dell'ospedale mentre EMPEROR-Preserved non le 
Tabella 2. Recenti studi clinici e trial in corso randomizzati controllati su HFpEF.

\begin{tabular}{lllll}
\hline Trial & Tipo & Classi farmaci & Farmaci & Studio completato \\
\hline PARAGON-HF & HFpEF & ARNI & Sacubitril/valsartan & 2019 \\
\hline EMPEROR-preserved & HFpEF & Inibitori del SGLT2 & Empaglifozin & 2021 \\
\hline DELIVER & HFpEF & Inibitori del SGLT2 & Dapaglifozin & 2021 \\
\hline SPIRRIT-HEPEF & HFpEF & Antagonisti Mineralcorticoidi & Spironolattone & 2022 \\
\hline
\end{tabular}

PARAGON-HF, Prospective Comparison of ARNI versus ACE inibitor Trial to Determine Superiority in Reducing Heart Failure Evenets After MI; EMPEROR-preserved, EMPaglifozin outcomE tRial in Patient With chrOnic heaRt Failure With Preserved Ejection Fraction. DELIVER, Dapagliflozin Evaluation to Improve the LIVEs of Patients With PReserved Ejection Fraction Heart Failure; SPIRRIT-HFPEF, Spironolactone Initiation Registry Randomized Interventional Trial in heart failure with Preserved Ejection Fraction.

inserisce nell'end-point primario) (Tabella 2). Esistono alcune differenze tra i due studi: nello studio DELIVER, i pazienti arruolati presentano scompenso cardiaco sintomatico da almeno 6 settimane mentre una durata di scompenso cardiaco sintomatico di almeno 12 settimane di scompenso sintomatico era richiesta per l'arruolamento nello studio EMPERORPreserved. In attesa di poter disporre dei risultati finali di questi studi, appare altrettanto fondamentale la necessità di comprendere l'effetto additivo degli inibitori SGLT2 con altre classi di farmaci nei pazienti con HFpEF.

\section{Conclusioni}

In relazione all'incremento della prevalenza di pazienti con HFpEF è fondamentale accrescere la conoscenza dell'armamentario terapeutico farmacologico e non farmacologico per poter interferire con lo sviluppo e la progressione di questa forma estremamente eterogenea di scompenso cardiaco. La gestione dei fattori di rischio associati, quali l'ipertensione arteriosa, il diabete mellito e l'obesità, la malattia renale cronica, appare un elemento fondante del piano di trattamento di questi pazienti. La possibilità, anche alla luce dei risultati dei più recenti studi clinici e di quelli ancora in corso (Tabella 2), di disporre di classi di farmaci quali antagonisti dei mineralcorticoidi, antagonisti del sistema reninaangiotensina/inibitori della neprilisina, e inibitori di SGLT2 rappresenta una sfida fondamentale considerando la frustrazione per la scarsità dei risultati osservati su questi pazienti nel corso delle ultime due decadi, cercando finalmente di colmare il gap di conoscenza che rappresenta un importante bisogno insoddisfatto in medicina interna e in cardiologia.

\section{Bibliografia}

1. Ather S, Chan W, Bozkurt B, et al. Impact of noncardiac comorbidities on morbidity and mortality in a predominantly male population with heart failure and preserved versus reduced ejection fraction. J Am Coll Cardiol. Am Coll Cardiol. 2012;59:998-1005.
2. van Riet EE, Hoes AW, Wagenaar KP, et al. Epidemiology of heart failure: the prevalence of heart failure and ventricular dysfunction in older adults over time. A systematic review. Eur J Heart Fail 2016;18:242-252.

3. Seferovic PM, Petrie MC, Filippatos GS, et al. Type 2 diabetes mellitus and heart failure: a position statement from the Heart Failure Association of the European Society of Cardiology. Eur J Heart Fail 2018;20:853-872.

4. Yancy CW, Lopatin M, Stevenson LW, et al. ADHERE Scientific Advisory Committee and Investigators. Clinical presentation, management, and in-hospital outcomes of patients admitted with acute decompensated heart failure with preserved systolic function: a report from the Acute Decompensated Heart Failure National Registry (ADHERE) Database. J Am Coll Cardiol. 2006; 47:76-84.

5. Streng KW, Nauta JF, Hillege HL, et al. Non-cardiac comorbidities in heart failure with reduced, mid-range and preserved ejection fraction. Int $\mathrm{J}$ Cardiol. 2018;271:132-139.

6. Tribouilloy C, Rusinaru D, Mahjoub H, et al. Prognosis of heart failure with preserved ejection fraction: a 5 year prospective population-based study. Eur Heart J. 2008;29:339-347.

7. Ho JE, Enserro D, Brouwers FP, et al. Predicting heart failure with preserved and reduced ejection fraction: The International Collaboration on Heart Failure Subtypes. Circ Heart Fail. 2016;9:e003116.

8. Gerber Y, Weston SA, Redfield MM, et al. A contemporary appraisal of the heart failure epidemic in Olmsted County, Minnesota, 2000 to 2010. JAMA Intern Med. 2015;175:996-1004.

9. Gottdiener JS, McClelland RL, Marshall R, et al. Outcome of congestive heart failure in elderly persons: influence of left ventricular systolic function. The Cardiovascular Health Study. Ann Intern Med. 2002;137:631-639.

10. Chang PP, Wruck LM, Shahar E, et al. Trends in hospitalizations and survival of acute decompensated heart failure in four US Communities (2005-2014): ARIC Study Community Surveillance. Circulation. 2018;138:12-24.

11. Gupta DK, Shah AM, Castagno D, et al. Heart failure with preserved ejection fraction in African Americans: The ARIC (Atherosclerosis Risk In Communities) study. JACC Heart Fail. 2013;1:156-163.

12. Lam CS, Donal E, Kraigher-Krainer E, Vasan RS. Epidemiology and clinical course of heart failure with preserved ejection fraction. Eur J Heart Fail. 2011;13:18-28. 
13. Hwang SJ, Melenovsky V, Borlaug BA. Implications of coronary artery disease in heart failure with preserved ejection fraction. J Am Coll Cardiol. 2014;63:2817-2827.

14. Borlaug BA, Anstrom KJ, Lewis GD, et al; National Heart, Lung, and Blood Institute Heart Failure Clinical Research Network. Effect of inorganic nitrite vs placebo on exercise capacity among patients with heart failure with preserved ejection fraction: The INDIEHFpEF randomized clinical trial. JAMA. 2018;320:1764-1773.

15. Obokata M, Reddy YNV, Pislaru SV, et al. Evidence supporting the existence of a distinct obese phenotype of heart failure with preserved ejection fraction. Circulation. 2017;136:6-19.

16. Echouffo-Tcheugui JB, $\mathrm{Xu} \mathrm{H}$, DeVore AD, et al. Temporal trends and factors associated with diabetes mellitus among patients hospitalized with heart failure: Findings from Get With The Guidelines-Heart Failure registry. Am Heart J. 2016; 182:9-20.

17. Rosano GM, Vitale C, Seferovic P. Heart Failure in Patients with Diabetes Mellitus. Card Fail Rev. 2017; 3(1):52-55.

18. Vasan RS, Larson MG, Benjamin EJ, et al. Congestive heart failure in subjects with normal versus reduced left ventricular ejection fraction: prevalence and mortality in a population-based cohort. J Am Coll Cardiol. 1999;33:1948-1955.

19. Owan TE, Hodge DO, Herges RM, et al. Trends in prevalence and outcome of heart failure with preserved ejection fraction. N Engl J Med. 2006;355:251-259.

20. Bhatia RS, Tu JV, Lee DS, et al. Outcome of heart failure with preserved ejection fraction in a populationbased study. N Engl J Med. 2006;355:260-269.

21. Kupari M, Lindroos M, Iivanainen AM, et al. Congestive heart failure in old age: prevalence, mechanisms and 4-year prognosis in the Helsinki Ageing Study. J Intern Med. 1997;241:387-394.

22. Lewis EF, Lamas GA, O'Meara E, et al; CHARM Investigators. Characterization of health-related quality of life in heart failure patients with preserved versus low ejection fraction in CHARM. Eur $\mathrm{J}$ Heart Fail. 2007;9:83-91.

23. Loop MS, Van Dyke MK, Chen L, et al. Comparison of length of stay, 30-day mortality, and 30-day readmission rates in medicare patients with heart failure and with reduced versus preserved ejection fraction. Am J Cardiol. 2016;118:79-85.

24. Nagueh SF. Heart Failure with Preserved Ejection Fraction: Insights into Diagnosis and Pathophysiology. Cardiovasc Res. 2020 :cvaa228.

25. Cabassi A, Miragoli M. Altered Mitochondrial Metabolism and Mechanosensation in the Failing Heart: Focus on Intracellular Calcium Signaling. Int J Mol Sci. 2017;18(7):1487

26. Pfeffer MA, Shah AM, Borlaug BA. Heart Failure with Preserved Ejection Fraction In Perspective. Circ Res. 2019;124(11):1598-1617.

27. Cabassi A, Binno SM, Tedeschi S, et al. Low serum ferroxidase I activity is associated with mortality in heart failure and related to both peroxynitrite-induced cysteine oxidation and tyrosine nitration of ceruloplasmin. Circ Res. 2014;114(11):1723-32.

28. Tedeschi S, Pilotti E, Parenti E, et al. Serum adipokine zinc $\alpha 2$-glycoprotein and lipolysis in cachectic and noncachectic heart failure patients: relationship with neurohormonal and inflammatory biomarkers. Metabolism. 2012;61(1):37-42.

29. Cabassi A, Tedeschi S. Zinc- $\alpha 2$-glycoprotein as a marker of fat catabolism in humans. Curr Opin Clin Nutr Metab Care. 2013;16(3):267-71.

30. Cabassi A, de Champlain J, Maggiore U, et al. Prealbumin improves death risk prediction of BNPadded Seattle Heart Failure Model: results from a pilot study in elderly chronic heart failure patients. Int $\mathrm{J}$ Cardiol. 2013;168(4):3334-9.

31. Franssen C, Chen S, Unger A, et al. Myocardial microvascular inflammatory endothelial activation in heart failure with preserved ejection fraction. JACC Heart Fail. 2016;4:312-324.

32. Cabassi A, Vinci S, Quartieri F, et al. Norepinephrine reuptake is impaired in skeletal muscle of hypertensive rats in vivo. Hypertension. 2001;37(2):698-702.

33. Cabassi A, Coghi P, Govoni P, et al. Sympathetic modulation by carvedilol and losartan reduces angiotensin II-mediated lipolysis in subcutaneous and visceral fat. J Clin Endocrinol Metab. 2005;90(5):2888-97.

34. Luptak I, Sverdlov AL, Panagia M, et al. Decreased ATP production and myocardial contractile reserve in metabolic heart disease. J Mol Cell Cardiol. 2018;116:106-114.

35. Caffarra Malvezzi C, Cabassi A, Miragoli M. Mitochondrial mechanosensor in cardiovascular diseases. Vasc Biol. 2020;2(1):R85-R92.

36. Alehagen U, Benson L, Edner M, et al. Association Between Use of Statins and Mortality in Patients With Heart Failure and Ejection Fraction of $\geq 50$. Circ Heart Fail. 2015;8(5):862-70.

37. Kitzman DW, Brubaker P, Morgan T, et al. Effect of caloric restriction or aerobic exercise training on peak oxygen consumption and quality of life in obese older patients with heart failure with preserved ejection fraction: a randomized clinical trial. JAMA. 2016;315:36-46.

38. Kitzman DW, Brubaker PH, Morgan TM, et al. Exercise training in older patients with heart failure and preserved ejection fraction: a randomized, controlled, single-blind trial. Circ Heart Fail. 2010;3(6):659-67.

39. Zakeri R, Chamberlain AM, Roger VL, Redfield MM. Temporal relationship and prognostic significance of atrial fibrillation in heart failure patients with preserved ejection fraction: a community-based study. Circulation. 2013;128(10):1085-93.

40. Mohammed SF, Hussain S, Mirzoyev SA, et al. Coronary microvascular rarefaction and myocardial fibrosis in heart failure with preserved ejection fraction. Circulation. 2015;131(6):550-9.

41. Hwang SJ, Melenovsky V, Borlaug BA. Implications of coronary artery disease in heart failure with preserved ejection fraction. J Am Coll Cardiol. 2014;63(25 Pt A):2817-27.

42. Ponikowski P, Voors AA, Anker SD, et al. 2016 ESC guidelines for the diagnosis and treatment of acute and chronic heart failure: the task force for the diagnosis and treatment of acute and chronic heart failure of the European Society of Cardiology (ESC). Developed with the special contribution of the Heart Failure 
Association (HFA) of the ESC. Eur J Heart Fail 2016;18:891-975.

43. Yancy CW, Jessup M, Bozkurt B, et al. 2017 ACC/AHA/HFSA Focused Update of the 2013 ACCF/AHA guideline for the management of heart failure: a report of the American College of Cardiology/American Heart Association Task Force on Clinical Practice Guidelines and the Heart Failure Society of America. Circulation. 2017;136:e137-e161.

44. Yusuf S, Pfeffer MA, Swedberg K, et al.; CHARM Investigators and Committees. Effects of candesartan in patients with chronic heart failure and preserved leftventricular ejection fraction: the CHARM-Preserved Trial. Lancet. 2003;362:777-781.

45. Massie BM, Carson PE, McMurray JJ, et al.; IPRESERVE Investigators. Irbesartan in patients with heart failure and preserved ejection fraction. N Engl J Med. 2008;359:2456-2467.

46. Cleland JG, Tendera M, Adamus J, et al.; PEP-CHF Investigators. The perindopril in elderly people with chronic heart failure (PEP-CHF) study. Eur Heart J. 2006;27:2338-2345.

47. Pfeffer MA, Claggett B, Assmann SF, et al. Regional variation in patients and outcomes in the Treatment of Preserved Cardiac Function Heart Failure With an Aldosterone Antagonist (TOPCAT) trial. Circulation. 2015;131:34-42.

48. Pitt B, Pfeffer MA, Assmann SF, et al; TOPCAT Investigators. Spironolactone for heart failure with preserved ejection fraction. N Engl J Med. 2014; 370:1383-1392.

49. Abraham WT, Adamson PB, Bourge RC, et al.; CHAMPION Trial Study Group. Wireless pulmonary artery haemodynamic monitoring in chronic heart failure: a randomised controlled trial. Lancet. 2011;377:658-666.

50. Solomon SD, McMurray JJV, Anand IS, et al.; PARAGON-HF Investigators and Committees. Angiotensin-Neprilysin Inhibition in Heart Failure with Preserved Ejection Fraction. N Engl J Med. 2019;381(17):1609-1620.

51. McMurray JJ, Packer M, Desai AS, et al.; PARADIGMHF Investigators and Committees. Angiotensin-neprilysin inhibition versus enalapril in heart failure. N Engl J Med. 2014 Sep 11;371(11):993-1004.

52. Abdul-Ghani M, Del Prato S, Chilton R, DeFronzo RA. SGLT2 inhibitors and cardiovascular risk: lessons learned from the EMPA-REG OUTCOME study. Diabetes Care. 2016;39:717-25.

53. Arnott C, Li Q, Kang A, et al. Sodium-glucose cotransporter 2 inhibition for the prevention of cardiovascular events in patients with type 2 diabetes mellitus: a systematic review and meta-analysis. J Am Heart Assoc. 2020;9:e014908.

54. Zinman B, Wanner C, Lachin JM, et al. Empagliflozin, cardiovascular outcomes, and mortality in type 2 diabetes. N Engl J Med 2015;373:2117-28.

55. Packer M, Anker SD, Butler J, Filippatos G, Zannad F.
Effects of sodium-glucose co-transporter 2 inhibitors for the treatment of patients with heart failure: proposal of a novel mechanism of action. JAMA Cardiol 2017;2:1025-1029.

56. Uthman L, Baartscheer A, Schumacher CA, et al. Direct cardiac actions of sodium glucose co-transporter 2 inhibitors target pathogenic mechanisms underlying heart failure in diabetic patients. Front Physiol 2018;9:1575.

57. Ojima A, Matsui T, Nishino Y, et al. Empagliflozin, an inhibitor of sodium-glucose co-transporter 2 exerts antiinflammatory and antifibrotic effects on experimental diabetic nephropathy partly by suppressing AGEsreceptor axis. Horm Metab Res 2015;47:686-692.

58. Packer M. Leptin-aldosterone-neprilysin axis: identification of its distinctive role in the pathogenesis of the three phenotypes of heart failure in people with obesity. Circulation 2018;137: 1614-1631.

59. Yagi S, Hirata Y, Ise T, et al. Canagliflozin reduces epicardial fat in patients with type 2 diabetes mellitus. Diabetol Metab Syndr. 2017;9:78.

60. Kusaka H, Koibuchi N, Hasegawa Y, et al. Empagliflozin lessened cardiac injury and reduced visceral adipocyte hypertrophy in prediabetic rats with metabolic syndrome. Cardiovasc Diabetol 2016;15:157.

61. Zhang Y, Nakano D, Guan Y, et al. A sodium-glucose co-transporter 2 inhibitor attenuates renal capillary injury and fibrosis by a vascular endothelial growth factor-dependent pathway after renal injury in mice. Kidney Int 2018;94:524-535.

62. Hallow KM, Gebremichael Y, Helmlinger G, Vallon V. Primary proximal tubule hyperreabsorption and impaired tubular transport counterregulation determine glomerular hyperfiltration in diabetes: a modeling analysis. Am J Physiol Renal Physiol 2017;312:F819-F835.

63. Neal B, Perkovic V, Mahaffey KW, et al. Canagliflozin and cardiovascular and renal events in type 2 diabetes. N Engl J Med 2017;377:644-57.

64. Cannon CP, Pratley R, Dagogo-Jack S, et al.; VERTIS $\mathrm{CV}$ Investigators. Cardiovascular Outcomes with Ertugliflozin in Type 2 Diabetes. N Engl J Med. 2020;383(15):1425-1435.

65. Wiviott SD, Raz I, Bonaca MP, et al.; DECLARE-TIMI 58 Investigators. Dapagliflozin and Cardiovascular Outcomes in Type 2 Diabetes. N Engl J Med. 2019;380(4):347-357.

66. McMurray JJV, Solomon SD, Inzucchi SE, et al.; DAPA-HF Trial Committees and Investigators. Dapagliflozin in Patients with Heart Failure and Reduced Ejection Fraction. N Engl J Med. 2019;381(21):1995-2008.

67. Packer M, Anker SD, Butler J, et al.; EMPERORReduced Trial Investigators. Cardiovascular and Renal Outcomes with Empagliflozin in Heart Failure. N Engl J Med. 2020;383(15):1413-1424.

68. Kato ET, Silverman MG, Mosenzon O, et al. Effect of dapagliflozin on heart failure and mortality in type 2 diabetes mellitus. Circulation. 2019;139: 2528-36. 


\title{
Scompenso cardiaco: rivalutazione della terapia in fase di ospedalizzazione
}

\author{
Francesco Corradi, Ilaria Lamberti, Giacomo Gelati, Simona Massini \\ U.O.C. Medicina Interna Casentino, Stabilimento Ospedaliero del Casentino, Presidio Ospedali Riuniti Aretino, Bibbiena (AR), \\ Italia
}

\section{Introduzione}

Il ricovero ospedaliero per scompenso cardiaco acuto rappresenta nel mondo occidentale una percentuale dei ricoveri totali pari all' $1-2 \% .{ }^{1}$ Come ormai è noto da tempo, il tasso di mortalità di questi pazienti è aumentato, sia in fase di ricovero sia nel post-dimissione, mentre le percentuali di riammissione ospedaliera variano dal 15 al $22 \%$ nel corso del primo mese, fino al $35-45 \%$ nel corso dei 3 mesi successivi. ${ }^{2}$ A ciò consegue un costo unitario per anno per paziente di circa 11.000 euro, di cui l' $85 \%$ per il ricovero $(1,4-2 \%$ della spesa complessiva del SSN). ${ }^{3}$ Lo scompenso cardiaco è, quindi, una sindrome clinica a larga prevalenza nella popolazione generale (2-3\%, pari ad oltre 1.000.000 di pazienti ogni anno), ancor più se consideriamo un campione di pazienti in età avanzata (10$20 \%$ nell'intervallo $70-80$ anni). La mortalità a 5 anni dal primo ricovero è pari al $40-50 \%$ di cui il $25 \%$ entro i primi 12 mesi. ${ }^{4}$ Inoltre, rispetto alla forma insorta de novo ( $44 \%$ dei casi), lo scompenso cardiaco acuto inteso come riacutizzazione di un'insufficienza cardiaca cronica $(56 \%)$, è una condizione più severa e associata ad una mortalità più elevata. ${ }^{5}$ Gli obiettivi di trattamento dello scompenso cardiaco cronico riacutizzato sono il sollievo dei sintomi, l'identificazione dei fattori precipitanti e il miglioramento della mortalità post-dimissione oltre che la prevenzione delle re-ospe-

Corrispondente: Francesco Corradi e Simona Massini, U.O.C. Medicina Interna Casentino, Stabilimento Ospedaliero del Casentino, Presidio Ospedali Riuniti Aretino, via Filippo Turati 55, 52011 Bibbiena (AR), Italia.

Tel.: +29.0575.568260 - 0575.568259.

E-mail: francescocorradi72@gmail.com ;

simona.massini@uslsudest.toscana.it

Articolo pubblicato secondo la Creative Commons Attribution NonCommercial 4.0 License (CC BY-NC 4.0).

${ }^{\circ}$ Copyright: the Author(s), 2021

Licensee PAGEPress, Italy

QUADERNI - Italian Journal of Medicine 2021; 9(2):e6 dalizzazioni. ${ }^{6}$ L'ospedalizzazione può rappresentare, in molti casi, un'opportunità per impostare o riconsiderare il trattamento farmacologico di un paziente con scompenso cardiaco. ${ }^{7}$ Come sappiamo, le modalità di presentazione clinica dello scompenso acuto possono variare a seconda dello stato di volemia e di congestione, realizzando così quadri clinici diversi, con prognosi molto diverse e ricadute evidenti sulle scelte terapeutiche da effettuare in fase acuta, post-acuzie e alla dimissione.

Il quadro del paziente warm and dry, caldo e asciutto (un terzo del totale) è quello associato alla prognosi migliore e spesso scatenato da un rialzo ipertensivo, pertanto la gestione di questi pazienti si incentra soprattutto sul trattamento antiipertensivo e sulla titolazione dei $\beta$-bloccanti e degli angiotensin converting enzyme-inhibitor (ACE-i). Circa il 50\% del totale è rappresentato dai pazienti warm and wet, caldo e umido, che necessitano sostanzialmente di un trattamento per la congestione e che quindi avranno una terapia incentrata essenzialmente sui diuretici.

Il gruppo cold and wet, freddo e umido (circa 20\% del totale) richiede una terapia basata sull'utilizzo di vasodilatatori inotropi per raggiungere un'adeguata perfusione e ridurre la congestione. Infine, il gruppo dei cold and dry, freddo e asciutto, rappresenta una percentuale minore di pazienti che non presentano sintomi e segni di congestione, a fronte tuttavia di una riduzione significativa della riserva cardiaca e che quindi necessitano di un supporto inotropo e di un attento riempimento volemico. Nel case mix di un reparto di Medicina Interna, lo scompenso cardiaco riveste un ruolo certamente importante. L'Osservatorio ARNO (osservatorio multicentrico delle prestazioni sanitarie erogate dal SSN al singolo cittadino) rilevava, già nel 2010 , che in oltre il $70 \%$ dei casi lo scompenso cardiaco acuto de novo o cronico riacutizzato era gestito da strutture internistiche o di geriatria. Rispetto agli studi clinici randomizzati-controllati, i più recenti studi di real world hanno dimostrato che il paziente con scompenso cardiaco presenta un'età più avanzata e un maggior numero di comorbilità, spostando l'attenzione su un paziente complesso e poli- 
patologico. ${ }^{8}$ Molti studi epidemiologici, tra cui il TEMISTOCLE e il CONFINE hanno evidenziato con chiarezza che in questa popolazione insistono una o più comorbilità (almeno due nell' $80 \%$ dei casi) in grado di pesare in modo significativo sulla mortalità e che solo nella metà circa delle re-ospedalizzazioni per scompenso cardiaco cronico è riconoscibile una causa cardiovascolare. ${ }^{9,10}$ Per comprendere il percorso di rivalutazione da effettuare in questi pazienti, il Medico Internista, al netto delle comorbilità, deve comprendere la causa che ha determinato lo scompenso che, almeno in parte, ne condizionerà le scelte terapeutiche.

\section{L'inquadramento del paziente con scompenso cardiaco: anamnesi e ricognizione farmacologica}

I sintomi dello scompenso cardiaco spesso non sono specifici (pensiamo ad esempio alla dispnea, che può riconoscere diverse cause) e possono essere anche difficili da riconoscere, insieme ai segni stessi, particolarmente in alcune categorie di pazienti, come ad esempio gli obesi o gli anziani o i respiratori cronici. Un'accurata anamnesi resta, quindi, una tappa fondamentale nel lavoro dell'internista, con particolare riferimento alla storia cardiovascolare (ipertensione, valvulopatie, storia di infarto miocardico, pregressa patologia tromboembolica), ma non solo. Si dovrà, infatti, prestare particolare attenzione anche all'eventuale presenza di patologie non cardiovascolari come neoplasie e chemiotrattamenti, abuso di sostanze, patologie metaboliche quali diabete, anemia, insufficienza renale, insufficienza epatica e patologie tiroidee. La dimostrazione di una causa cardiaca nel contesto del quadro clinico è di fondamentale importanza nella diagnosi di scompenso, così come la valutazione della funzione sistolica che permette l'iniziale inquadramento del paziente nel gruppo heart failure reduced ejection fraction (HFrEF) con $\mathrm{EF}<40 \%$ o nel gruppo heart failure preserved ejection fraction (HFpEF) con $\mathrm{EF} \geq 50 \%$. Questa suddivisione ha valore prognostico e implicazioni terapeutiche, in quanto, come sappiamo, è solo nel gruppo HFrEF che le terapie degli ultimi anni hanno dimostrato di ridurre mortalità e morbilità all'interno dei randomized controlled trial (RCT). La diagnosi invece di HFpEF è molto più impegnativa, non essendo associata ad una disfunzione ventricolare sinistra, ma piuttosto ad un aumento delle pressioni di riempimento e quindi ad una disfunzione diastolica. La terza categoria poi, heart failure mid range ejection fraction (HFmEF), con EF tra 40 e $49 \%$ è stata riconosciuta come entità nosologica solo dalla ultime linee guida, con lo scopo di stimolare maggiormente la ricerca delle cause sottostanti, la comprensione dei meccanismi fisiopatologici e il trattamento. ${ }^{1}$ Alla raccolta anamnestica e all'inquadramento clinico seguirà la ricognizione farmacologica, cioè la verifica di tutte le terapie farmacologiche e non che il paziente stava assumendo prima del ricovero. Questa fase, troppo spesso sottovalutata, è invece estremamente importante perché ci consente di identificare casi, purtroppo molto frequenti, nei quali le terapie farmacologiche adottate sono ampiamente sottodosate (non raggiungono cioè le dosi target indicate dalle linee guida) ma anche quei casi in cui non compaiono classi di farmaci di comprovata efficacia.

\section{La visione olistica del paziente con scompenso cardiaco: comorbilità, complessità e appropriatezza terapeutica}

I pazienti ricoverati nei reparti di Medicina Interna per scompenso cardiaco manifestano un grado di complessità assai elevato, non solo per l'età mediamente più avanzata, ma anche per la presenza, concomitante, di una o più comorbilità che si traduce in un numero più elevato di giornate di degenza e in una più elevata mortalità intraospedaliera. ${ }^{11,12} \mathrm{Nel} 2019$, l'American College of Cardiology, ${ }^{13}$ pubblica un documento di consenso per la gestione del paziente ospedalizzato con scompenso cardiaco classificando le comorbilità in cardiovascolari, patologie sistemiche e psicosociali. Le comorbilità si comportano in molti casi come fattori precipitanti uno scompenso cardiaco cronico e pertanto devono rappresentare un target di intervento terapeutico specifico e indipendente. Inoltre, la loro presenza, induce sintomi più severi, una maggior limitazione funzionale ed una qualità di vita peggiore (Figure 1 e 2).

La storia naturale di questa malattia cronica si caratterizza per episodi di acuzie in grado di aumentare la fragilità di questi pazienti esponendoli a potenziali e ripetuti ricoveri. Se da una parte il ricovero per scompenso cardiaco acuto ci costringe ad un trattamento d'urgenza con il fine di ottenere il più rapidamente possibile un nuovo equilibrio emodinamico e la risoluzione del quadro congestizio, dall'altra ci regala un'occasione per rivalutare la terapia in atto, l'aderenza al trattamento, l'eventuale raggiungimento delle dosi target, un potenziale upgrade non precedentemente contemplato e l'identificazione di comorbilità potenzialmente correggibili per una complessiva stratificazione del rischio.

Poiché, in effetti, le comorbilità possono influenzare negativamente la terapia specifica dello scompenso, la possibilità di revisione delle classi di farmaci impiegate o eventuali incrementi del dosaggio, trovano nel ricovero un'occasione propizia per essere testati e monitorati in un ambiente sicuro. 


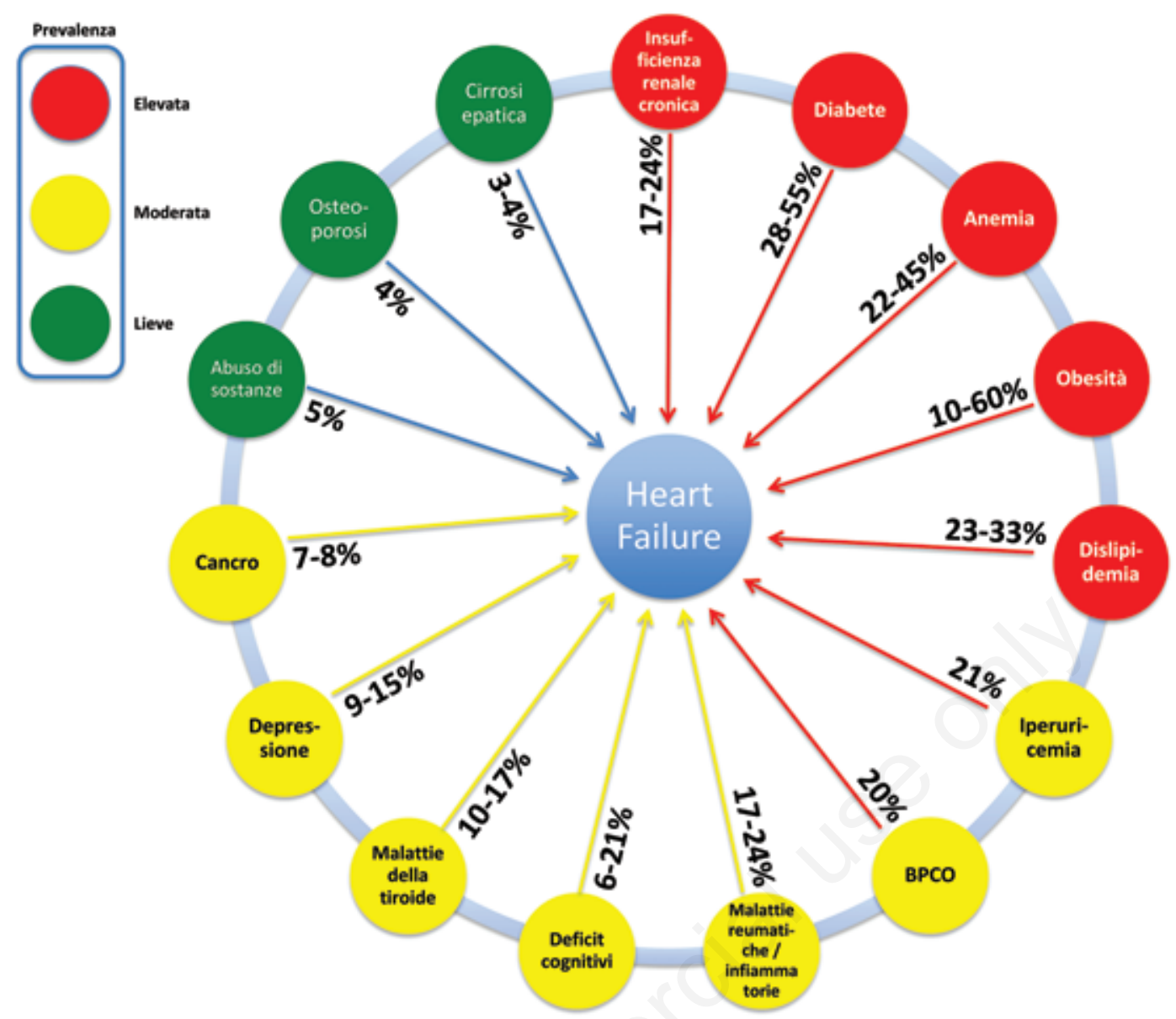

Figura 1. Prevalenza delle comorbilità non cardiovascolari nella popolazione di pazienti affetti da scompenso cardiaco.

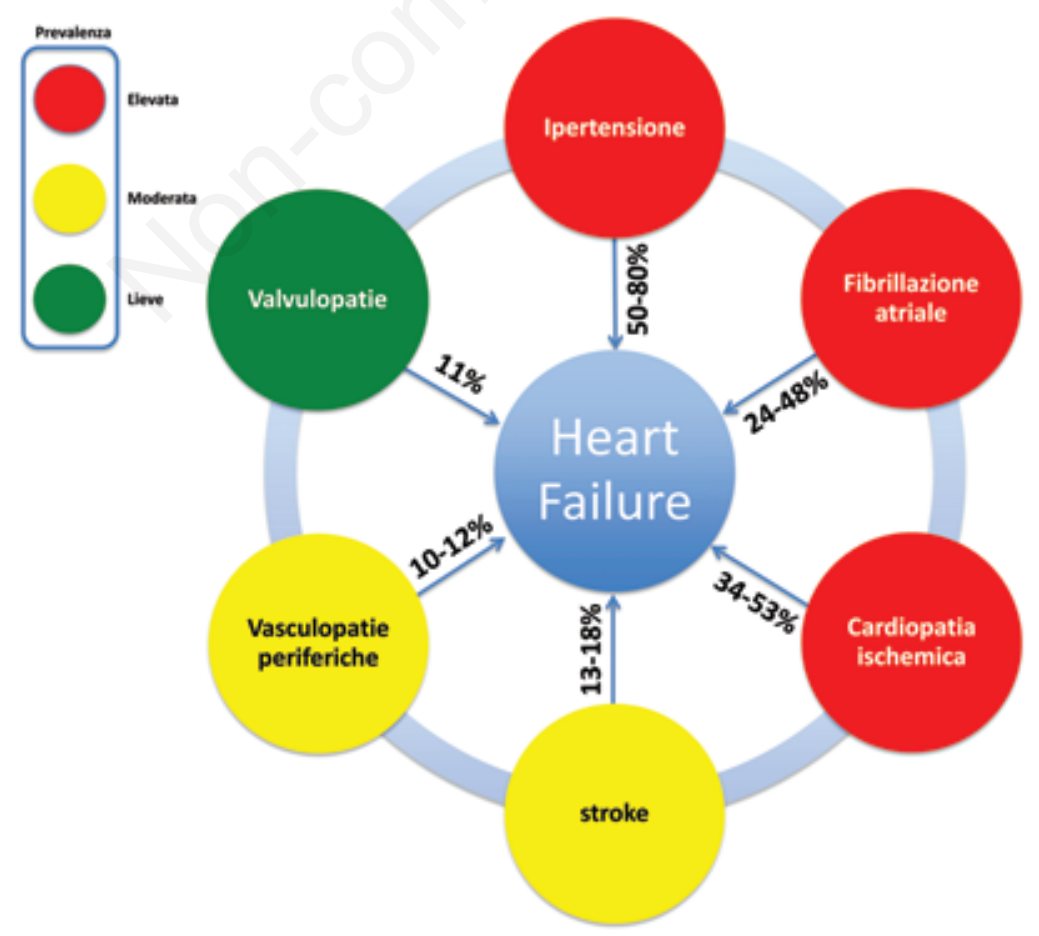

Figura 2. Prevalenza delle principali comorbilità cardiovascolari nella popolazione di pazienti affetti da scompenso cardiaco. 
Nei pazienti in età avanzata, affetti da scompenso cardiaco, la prevalenza di comorbilità può raggiungere anche il 96\% dei casi (circa l' $86 \%$ almeno 2 comorbilità, il $42 \%$ uguale o più di 5 ). ${ }^{14}$ Le età più avanzate presentano un numero maggiore di comorbilità ed una classe New York Heart Association (NYHA) più elevata. Oltre il $50 \%$ dei pazienti con scompenso cardiaco ricoverati in Medicina Interna presenta una funzione sistolica preservata. Questo tipo di scompenso cardiaco si ritrova con maggior frequenza nel sesso femminile, in età più avanzata e si correla, rispetto a quello a funzione sistolica ridotta, a comorbilità di tipo non necessariamente cardiovascolari che sono presenti in oltre il $30 \%$ dei casi. ${ }^{15}$ Tra le comorbilità non cardiovascolari, la malattia renale cronica ricopre un ruolo di primo piano, essendo presente in almeno la metà dei casi e va considerata, a tutti gli effetti, un fattore di rischio indipendente per scompenso cardiaco acuto. D'altra parte è ampiamente nota la stretta correlazione esistente tra cuore e rene, già esaurientemente codificata nella sindrome cardiorenale o reno-cardiaca. ${ }^{16} \mathrm{Si}$ stima che circa il $40 \%$ dei pazienti con scompenso cardiaco si ospedalizza in presenza di una riacutizzazione di una comorbilità non cardiovascolare e non per uno scompenso cardiaco acuto. Tra le comorbilità prevalenti si distinguono l'insufficienza renale, il diabete, lo stroke, la BPCO e l'anemia. Diversamente, pazienti affetti da $\mathrm{HFrEF}$, presentano un numero inferiore di comorbilità specie non cardiovascolari, fatta eccezione per l'insufficienza renale che si correla in modo statisticamente significativo ad entrambi i tipi. ${ }^{17} \mathrm{La}$ correlazione fra HFpEF e le comorbilità non cardiovascolari tipiche del paziente in età avanzata si spiega, semplicemente, con il fatto che esse sono preesistenti; diversamente, nel paziente con HFrEF, le comorbilità come insufficienza renale, stroke o anemia, sono per lo più una conseguenza della patologia cardiaca stessa. ${ }^{18,19}$ È stato ipotizzato che le comorbilità nel paziente con HFpEF, proprio perché preesistenti, inducano e sostengano nel tempo uno stato pro-infiammatorio cronico in grado di favorire vere e proprie alterazioni strutturali e funzionali dei miociti fino ad assumere dignità di cardiopatia. Ciò spiegherebbe perché il trattamento efficace delle comorbilità possa riflettersi positivamente sull'equilibrio emodinamico e sullo scompenso cardiaco cronico. ${ }^{20}$ Semplificando, quindi, il ricovero, rappresenta un percorso intraospedaliero di cura che inizia con il trattamento/risoluzione della fase acuta fino al raggiungimento di una nuova stabilizzazione clinicoemodinamica, prosegue con l'inquadramento classificativo dello scompenso cardiaco, l'identificazione e l'eventuale trattamento delle cause e delle comorbilità terminando con la rivalutazione della terapia di prima linea (Figura 3).

\section{Terapia e dose target: l'ospedalizzazione come occasione di revisione della terapia alla luce delle linee guida vigenti}

Le pietre miliari del trattamento dello scompenso cardiaco cronico sono rappresentate dagli ACE-i, dagli angiotensin ii receptor blocker (ARB), dai mineralcorticoid receptor antagonists (MRAs) e dai $\beta$-bloccanti (BB), tutti farmaci che agiscono inibendo l'iperattivazione neurormonale, estremamente dannosa e tipica della sindrome, e che hanno dimostrato di ridurre significativamente la mortalità e la morbilità nei pazienti con HFrEF (non invece nei pazienti con $\mathrm{HFpEF}$ ). Questi farmaci inoltre, insieme ai diuretici, riducono precarico e postcarico, con tutti gli effetti fisiopatologici che ne conseguono (aumento della gittata cardiaca, riduzione della domanda di ossigeno, tolleranza allo sforzo, ecc.). Più recentemente gli angiotensin receptor-neprilysin inhibitors (ARNI) hanno dimostrato superiorità rispetto agli $\mathrm{ACE}-\mathrm{i}$ nel ridurre il rischio di morte e il rischio di ospedalizzazione per scompenso, guadagnandosi meritatamente una sempre maggiore attenzione nel panorama farmacologico. $\mathrm{La}$ gestione della terapia orale dello scompenso durante la fase acuta di re-ospedalizzazione ha generato in passato non pochi dubbi e perplessità, in particolare in relazione alla possibilità di interromperla o di andare incontro ad una riduzione dei dosaggi, tenendo presente, tra l'altro, la possibilità di un ridotto assorbimento durante la fase acuta. ${ }^{2}$ Gli ACE-i hanno ormai ampiamente dimostrato di ridurre mortalità e morbilità in questi pazienti, e sono sempre raccomandati con un livello di evidenza IA, a meno che controindicati o scarsamente tollerati (grave insufficienza renale, tosse o angioedema). Devono essere titolati al massimo dosaggio tollerato, anche nei pazienti con disfunzione sistolica ventricolare sinistra asintomatica, per ridurre il rischio di sviluppo di scompenso, ospedalizzazione e morte per scompenso. ${ }^{5}$

I sartanici non hanno dimostrato in maniera consistente di ridurre la mortalità in pazienti con HErEF e quindi il loro uso dovrebbe essere considerato solo per i pazienti intolleranti agli ACE-i. Il livello di evidenza con cui vengono raccomandati è I B.

Anche i $\beta$-bloccanti, come gli ACE-i, hanno mostrato di ridurre mortalità e morbilità in pazienti sintomatici con HFrEF, nonostante il trattamento con un ACE-i; il livello di evidenza con cui sono raccomandati dalle linee guida europee e americane è sempre un livello I A. ${ }^{1}$ Vi è infatti consenso riguardo al fatto che queste due categorie di farmaci siano complementari e che possano essere iniziati in contemporanea subito dopo la diagnosi, con l'unico accorgimento che prevede di iniziare i $\beta$-bloccanti in pazienti stabilizzati, al minimo dosaggio, per poi titolarli al massimo dosaggio tollerato, cioè quando la fase iniziale di mag- 
giore congestione si è risolta. Questi farmaci hanno inoltre un altro campo di applicazione in quei pazienti con fibrillazione atriale in cui sia necessario un controllo della frequenza cardiaca e, soprattutto, nei pazienti con cardiopatia ischemica e con disfunzione sistolica ventricolare sinistra asintomatica, per ridurre il rischio di morte. Gli MRA, come spironolactone ed eplerenone, sono raccomandati in tutti i pazienti con HFrEF che restino ancora sintomatici nonostante il trattamento con ACE-i e $\beta$-bloccante, per ridurre il rischio di morte e di ospedalizzazione per scompenso. Anche in questo caso il livello di evidenza è IA (Tabella 1$){ }^{6}$

I diuretici rappresentano la principale linea di trattamento della fase acuta decompensata dello scompenso cronico; consentono di ottenere un sollievo dai sintomi di sovraccarico e di scarsa tolleranza allo sforzo, riducendo il precarico. Tuttavia il loro effetto sulla mortalità e sulla morbilità non è ancora stato studiato all'interno di RCT. Una metanalisi di Cochrane ha mostrato che in pazienti con scompenso cardiaco cronico, i diuretici dell'ansa e i diuretici tiazidici sembrano ridurre il rischio di morte e di peggioramento dello scompenso, oltre che migliorare la capacità di esercizio. ${ }^{21}$ Lo scopo della terapia diuretica è quello di raggiungere e di mantenere, con la minima dose possibile, uno stato euvolemico. Nelle fasi acute la via di somministrazione da scegliere è ovviamente quella endovenosa in quanto l'assorbimento orale del farmaco risulta ridotto a causa dell'edema intestinale. I dosaggi sono, in genere, nettamente maggiori rispetto a quelli assunti per via orale dal paziente in fase di stabilità clinica; si è visto infatti come dosaggi anche doppi all'abituale terapia orale producono sul piano clinico effetti maggiori sulla diuresi con conseguente perdita di peso e sollievo dai sintomi rispetto ad una strategia basata su dosaggi simili a quelli assunti oralmente. ${ }^{22}$ I diuretici a dosaggi più elevati utilizzati per via endovenosa in fase acuta non portano ad un deterioramento a lungo termine della funzionalità renale, anche se si può osservare un transitorio peggioramento nelle prime ore. ${ }^{23}$ Dosi generose e ripetute di diuretici dell'ansa possono portare al fenomeno della riduzione dell'efficacia diuretica (resistenza ai diuretici) e conseguente persistenza di segni e sintomi di congestione. Dosi crescenti di farmaco, e la possibilità di aggiungere diuretici di altre classi inducendo blocco sequenziale del nefrone permettono di eludere questo
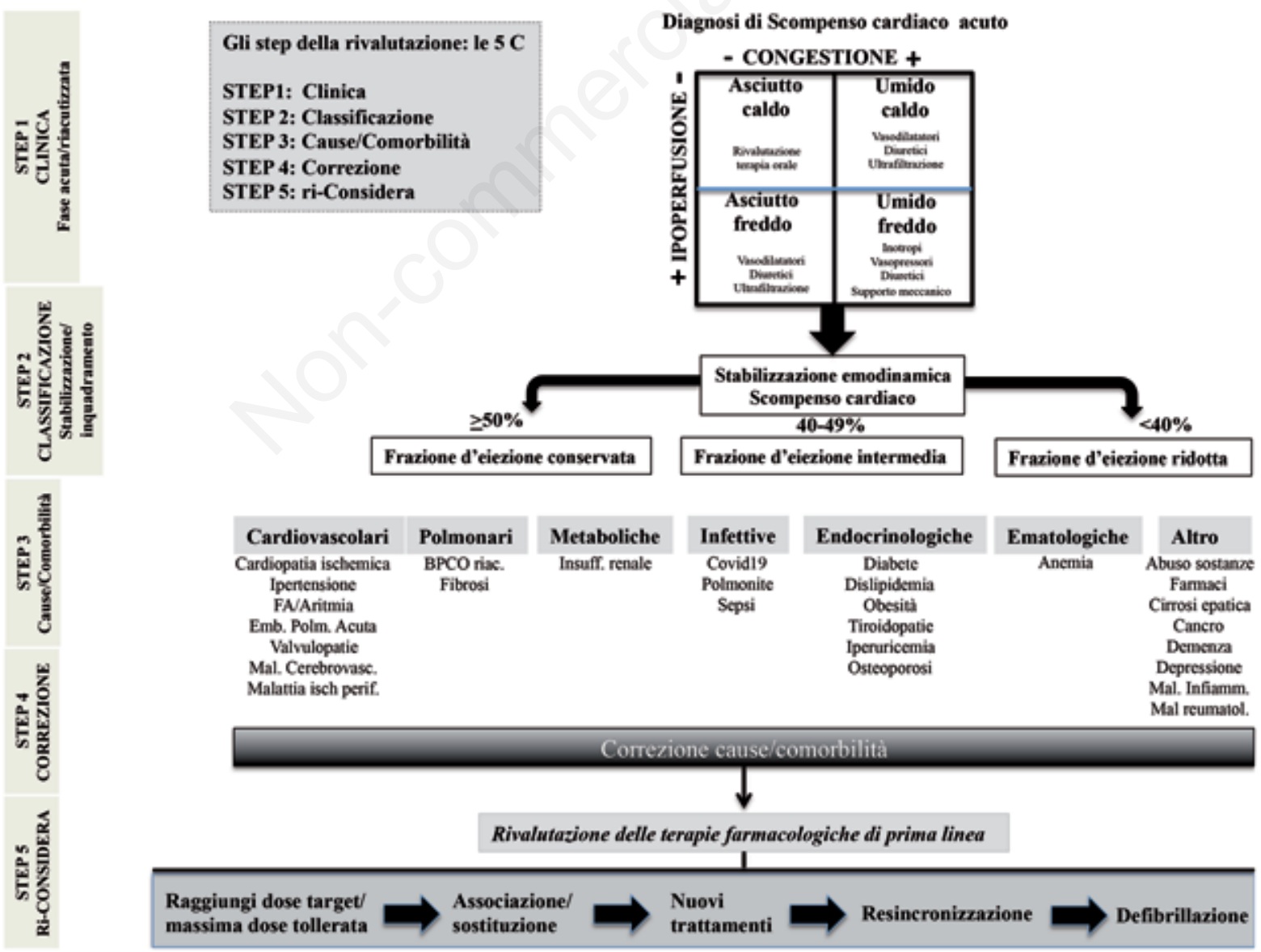

Figura 3. Gli step diagnostici e terapeutici nella valutazione del paziente con scompenso cardiaco. 
fenomeno. ${ }^{24}$ Farmaci quindi come idroclorotiazide o metolazone (i più utilizzati in Italia della categoria), somministrati per os, vanno ad aggiungersi alla terapia con furosemide ev, producendo un netto miglioramento in termini di diuresi, sollievo dai sintomi di congestione, della qualità di vita, di riduzione del peso corporeo, della durata dell'ospedalizzazione, consentendo di mantenere pazienti ambulatoriali in terapia orale o di ottenere la dimissibilità in casi particolarmente difficili. ${ }^{25,26} \mathrm{Ne}$ consegue che in caso di mancata responsività al metolazone $\mathrm{o}$ ai diuretici tiazidici la prognosi risulta particolarmente severa. ${ }^{27} \mathrm{I}$ regimi di trattamento consistono in bassi dosaggi di agenti a lunga durata d'azione (metolazone $2,5 \mathrm{mg} / \mathrm{die}$ ), da utilizzarsi 2-3 vv/settimana; mentre un regime più aggressivo (metolazone $10 \mathrm{mg} / \mathrm{die}$ per $2-3 \mathrm{gg}$ consecutivi) può essere efficace e sicuro, sempre monitorando gli elettroliti e la funzionalità renale, in caso di paziente ospedalizzato.

\section{Focus su nuovi trattamenti e terapie non farmacologiche}

Tra le novità in campo terapeutico, è ormai entrato nella pratica clinica il farmaco sacubitril-valsartan, primo agente della classe di farmaci chiamati ARNI, approvato dalla FDA per il trattamento di pazienti con HFrEF con classe NYHA II, III o IV.

Nello studio PARADIGM-HF sacubitril/valsartan si è dimostrato superiore ad enalapril in pazienti ambulatoriali con HFrEF, dimostrando di ridurre la mortalità cardiovascolare e l'ospedalizzazione per scompenso cardiaco di circa il $20 \%$, con un profilo di sicurezza e di tollerabilità paragonabile ad enalapril. ${ }^{28}$ L'inizio di sacubitril/valsartan è raccomandato già durante la fase di ospedalizzazione, in accordo con lo studio TRANSITION ${ }^{29}$ che ha confermato la tollerabilità del farmaco iniziato in pre-dimissione con una proporzione simile (circa la metà) di pazienti che già a 10 settimane avevano raggiunto la dose target. Sacubutril/valsartan può essere utilizzato al posto di un ACE-i o ARB nei pazienti emodinamicamente stabili, con ridotta frazione d'eiezione $(\mathrm{EF}<40 \%)$, che rimangono sintomatici, in aggiunta ad altre terapie raccomandate per lo scompenso cardiaco già a dose target (BB, MRA). ${ }^{30}$ Sono criteri di inclusione l'assenza di una storia di angioedema, la presenza di una velocità di filtrazione glomerulare $(\mathrm{VFG}) \geq 30$ $\mathrm{mL} / \mathrm{min} / 1,73 \mathrm{~m}^{2}$ e di valori di kaliemia $\leq$ a 5,2 $\mathrm{mEq} / \mathrm{L}$. Qualora tali criteri risultino soddisfatti, si procederà a sospensione di ACE-i almeno 36 ore prima, o alla sospensione di ARB (tranne valsartan) 24 ore prima. Raggiunta la stabilizzazione emodinamica, definita dallo studio PIONEER-HF ${ }^{31}$ come una pressione arteriosa $\geq 100 \mathrm{mmHg}$ senza aumento della dose di diuretici e di vasodilatatori nelle ultime 6 ore, e senza terapia inotropa nelle precedenti 24, sacubitril/valsartan potrà essere iniziato e titolato in ospedale con posologia $24 / 26 \mathrm{mg}$ $1 \mathrm{cp}$ bis in die (bid) qualora la pressione arteriosa sistolica per almeno 6 ore sia compresa tra 100 e 120 $\mathrm{mmHg}$, o $49 / 51 \mathrm{mg} 1 \mathrm{cp}$ bid, qualora questa sia $>120$ $\mathrm{mmHg}$. La presa in carico del paziente postdimissione da parte dell'ambulatorio dello scompenso cardiaco permetterà il successivo follow

Tabella 1. Farmaci più comunemente impiegati nel trattamento dello scompenso cardiaco cronico e relativi alert.

\begin{tabular}{|c|c|c|c|}
\hline ACE-i & Dose iniziale & Dose target & Alert \\
\hline Enalapril & $2,5 \mathrm{mg}$ bid & $10-20 \mathrm{mg}$ bid & \multirow[t]{3}{*}{ Insufficienza renale, gravidanza, iperkaliemia } \\
\hline Lisinopril & $2,5-5 \mathrm{mg}$ od & $20-35 \mathrm{mg}$ od & \\
\hline Ramipril & $2,5 \mathrm{mg} \mathrm{mg}$ od & $10 \mathrm{mg}$ od & \\
\hline$\beta$-bloccanti & Dose iniziale & Dose target & Alert \\
\hline Bisoprololo & $1,25 \mathrm{mg}$ od & $10 \mathrm{mg}$ od & \multirow[t]{4}{*}{ Bradicardia } \\
\hline Carvedilolo & $3,125 \mathrm{mg}$ bid & $25 \mathrm{mg}$ bid & \\
\hline Metoprololo & $12,5-25 \mathrm{mg}$ od & $200 \mathrm{mg}$ od & \\
\hline Nebivololo & $1,25 \mathrm{mg}$ od & $10 \mathrm{mg}$ od & \\
\hline MRAs & Dose iniziale & Dose target & Alert \\
\hline Eplerenone & $25 \mathrm{mg}$ od & $50 \mathrm{mg}$ od & \multirow[t]{2}{*}{ Insufficienza renale, iperkaliemia } \\
\hline Spironolactone & $25 \mathrm{mg}$ od & $50 \mathrm{mg}$ od & \\
\hline Diuretici & Dose iniziale & Dose target & Alert \\
\hline Furosemide & $20-40 \mathrm{mg}$ & $40-240 \mathrm{mg}$ & \multirow[t]{5}{*}{ Insufficienza renale, disionie } \\
\hline Torasemide & $5-10 \mathrm{mg}$ & $10-20 \mathrm{mg}$ & \\
\hline Idroclorotiazide & $25 \mathrm{mg}$ & $12,5-100 \mathrm{mg}$ & \\
\hline Metolazone & $2,5 \mathrm{mg}$ & $2,5-10 \mathrm{mg}$ & \\
\hline Indapamide & $2,5 \mathrm{mg}$ & $2,5-5 \mathrm{mg}$ & \\
\hline
\end{tabular}

ACE-i, angiotensin converting enzyme - inhibitor; MRA, mineralcorticoid receptor antagonist. 
up, la titolazione della dose (dalle 2 alle 4 settimane) e l'effettiva efficacia del farmaco entro le 8 settimane. Diventa, pertanto, essenziale, selezionare i pazienti realmente candidabili al trattamento con sacubitril/valsartan. Tali pazienti vanno ricercati tra coloro che si ospedalizzano, ma per i quali lo scompenso rappresenta solo una comorbilità e non la malattia indice causa del ricovero, e in cui un'attenta rivalutazione anamnestica, clinica e strumentale riveli l'insufficienza delle terapie, a dose target, già in atto. Devono essere ricercati inoltre tra coloro che, ospedalizzati per una riacutizzazione per scompenso cardiaco, mostrino un rapido raggiungimento di una nuova stabilizzazione emodinamica e per i quali siano possibili la riduzione/sospensione del diuretico e l'upgrade terapeutico con sacubitril/valsartan con le modalità suddette. Infine, vanno ricercati tra coloro che, dopo un'iniziale stabilizzazione che richiede tuttavia ancora trattamento diuretico, siano candidabili all'inserimento nel percorso ambulatoriale internistico di follow up, per poter effettuare l'upgrade terapeutico secondo quanto indicato dalle linee guida.

HFpEF $(\geq 50 \%)$, non ha ancora una terapia medica efficace, poiché nessun farmaco è riuscito a migliorare l'endpoint sopravvivenza in modo significativo. L'eccezione risulterebbe, in questa popolazione di pazienti, tra i $\beta$-bloccanti, solo il nebivololo, che ha dato qualche risultato positivo in termini di mortalità e ospedalizzazioni. In particolare, alcuni dati suggeriscono che l'effetto di nebivololo è simile nei pazienti con EF conservata e EF depressa. ${ }^{32}$ Nebivololo è un $\beta$-bloccante $\beta$ - 1 altamente selettivo, con un aggiuntivo effetto vasodilatatore mediato dall'ossido nitrico. ${ }^{33}$ L'effetto favorevole sarebbe dovuto proprio all'aumentato rilascio di ossido nitrico, che potrebbe incoraggiare il rilasciamento precoce del ventricolo sinistro, ${ }^{34}$ caratteristica questa che differenzierebbe nebivololo dagli altri $\beta$-bloccanti comunemente utilizzati nello scompenso cardiaco, come bisoprololo, metoprololo, carvedilolo.

Ci sono, poi, due altre caratteristiche meritevoli di essere discusse. La prima riguarda la capacità di questo farmaco di contrastare lo stress ossidativo e, conseguentemente, il danno e la disfunzione d'organo, tipica della cardiopatia ipertensiva. ${ }^{35} \mathrm{La}$ seconda riguarda la capacità del nebivololo di ridurre l'ipertrofia ventricolare sinistra (predittiva di un aumento di mortalità indipendentemente da altri fattori di rischio e induttore di fibrillazione atriale di nuova insorgenza), effetto ancora più spiccato se associato ad un ACE-i. ${ }^{36} \mathrm{Si}$ potrebbe speculare, quindi, che $\mathrm{i}$ meccanismi d'azione suddetti ci permettano di candidare nebivololo come scelta alternativa, ma razionale, nello $\mathrm{HFpEF}$, specie se conseguente a cardiopatia ipertensiva/diabetica ovvero a forme nelle quali l'ipertrofia ventricolare sinistra sia l'alterazione strutturale predominante.

Tra i farmaci degni di nota, poiché innovativi, trovano indicazione anche gli inibitori del cotrasportatore sodio-glucosio (sodium-glucose cotrasporter2 inhibitors, SGLT2), annoverati nella famiglia dei nuovi antidiabetici orali, anche noti come glicosurici che, tuttavia, forti evidenze hanno proposto anche nel trattamento dello scompenso cardiaco cronico.

Il razionale del loro utilizzo nello scompenso cardiaco è rappresentato dall'evidenza di una significativa riduzione delle gravi complicanze cardiovascolari e renali ad essa correlate, ed in particolar modo dalla riduzione, non solo della progressione della nefropatia diabetica verso il grado più avanzato di insufficienza renale che richieda la dialisi, ma anche del rischio di morte cardiovascolare e di ospedalizzazione nei pazienti con scompenso cardiaco. ${ }^{37,38}$ Gli effetti di protezione cardiovascolare e renale rilevati negli studi non sembrano essere direttamente correlati al controllo glicemico, suggerendo che tali benefici possano essere presenti anche nei pazienti non diabetici. ${ }^{39}$ Per tale motivo, possono essere inclusi nel trattamento, pazienti di almeno 18 anni di età con $\mathrm{HFrEF}<40 \%$ con classe NYHA II, III o IV, in presenza o meno di diabete mellito di tipo 2 , mentre dovranno essere esclusi pazienti affetti da diabete mellito di tipo 1, con ipotensione (pressione arteriosa sistolica inferiore a $95 \mathrm{mmHg}$ ) ancor più se sintomatica ed una VFG stimata $30 \mathrm{~mL} / \mathrm{min} / 1,73 \mathrm{~m}^{2}$ (o rapida riduzione della funzione renale).

Il paziente, eventualmente candidabile ad una terapia di associazione con questi farmaci, dovrà essere già in terapia farmacologica standard dello scompenso cardiaco con ACE-i, ARB o sacubitril/valsartan associati a BB e MRA, e in terapia non farmacologica con device (defibrillatore-cardiovertitore impiantabile, terapia di resincronizzazione, o entrambe). A partire dal 2015 sono stati presentati i risultati di studi clinici di intervento in pazienti diabetici con i tre farmaci della classe delle gliflozine: EMPAREG-outcome, ${ }^{37}$ CANVAS, ${ }^{38}$ DECLARETIMI58. In questi studi è stata dimostrata un'evidente efficacia rispettivamente di empagliflozin, canagliflozin e dapagliflozin sulla riduzione degli endpoints renali e cardiovascolari maggiori ed in particolare sull' endpoint di riduzione delle ospedalizzazioni e mortalità per scompenso cardiaco. Nei trials clinici di fase III DAPA-HF ed EMPEROR-reduced, dapagliflozin ha dimostrato di ridurre sia l'incidenza di morte per causa cardiovascolare sia la riacutizzazione e ospedalizzazione dello scompenso cardiaco in pazienti con HFrEF (classe NYHA II-IV), con e senza diabete mellito. L'Agenzia europea per i medicinali, 1'European Medical Agency (EMA), ha al 
momento approvato per la commercializzazione canagliflozin, dapagliflozin, empaglifozin.

Tra le proposte non farmacologiche dello scompenso cardiaco, possono essere d'ausilio procedure invasive, quali la chirurgia valvolare, la rivascolarizzazione miocardica, l'elettrostimolazione (pacemaker), il defibrillatore-cardiovertitore impiantabile, la resincronizzazione cardiaca, l'ultrafiltrazione, fino all'assistenza meccanica come terapia ponte in attesa di trapianto cardiaco in casi altamente selezionati. Il ricorso alla sostituzione valvolare per via percutanea o tramite chirurgia tradizionale, risulta necessario qualora l'insufficienza o la stenosi della valvola in questione sia tale da determinare un sovraccarico cronico del ventricolo sinistro, talora con ripercussioni emodinamiche significative anche sul cuore destro a cui possono seguire alterazioni strutturali irreversibili che peraltro renderebbero vani i suddetti interventi. Nel caso di specie, pertanto, l'individuazione precoce e l'attento monitoraggio clinico/strumentale diviene essenziale per stabilire la corretta tempistica d'intervento. ${ }^{40}$ La rivascolarizzazione coronarica si rende necessaria nei pazienti che presentano l'ischemia miocardica quale causa dello scompenso cardiaco. Questo sia nel caso in cui si tratti di angioplastica primaria e, quindi, di una vera e propria emergenza, sia nel caso di stenosi coronariche critiche che determinino un'ischemia cronica, ma in zone di miocardio vitale che si gioverebbero quindi di una rivascolarizzazione elettiva. ${ }^{41}$ Indicazioni all'impianto del pacemaker sono il blocco atrio-ventricolare di secondo grado tipo 2 e terzo grado, permanente o parossistico, sintomatico o asintomatico, disturbi del nodo del seno, bradicardia sintomatica sia in ritmo sinusale che in fibrillazione atriale, pause asistoliche superiori a 3 secondi, documentate. ${ }^{42}$

L'impianto di un defibrillatore-cardiovertitore trova indicazione nei pazienti con dilatazione del ventricolo sinistro ed $\mathrm{EF} \leq 35 \%$, che può esporre all'insorgenza di aritmie ventricolari maligne con aumentato rischio di morte cardiaca improvvisa, oltre che di progressione del deficit emodinamico. I defibrillatori impiantabili possono essere utilizzati sia in pazienti che hanno già subito una aritmia ventricolare grave (prevenzione secondaria) sia nei pazienti ad alto rischio di svilupparla (prevenzione primaria). ${ }^{43}$

La resincronizzazione cardiaca è in grado di migliorare la perfomance cardiaca, la qualità di vita ${ }^{44} \mathrm{e}$ la prognosi in termini di morbilità e mortalità. Essa è indicata nei pazienti con scompenso cardiaco in classe NYHA III-IV, EF $\leq 35 \%$, trattamento farmacologico ottimizzato, ritmo sinusale con una durata del QRS $\geq 150$ ms o durata del QRS tra 130 e $149 \mathrm{~ms}$ associata ad alterazioni della sincronia di contrazione (blocco di branca sinistra) evidenziate con ecocardiografia. ${ }^{45}$

L'ultrafiltrazione può essere utilizzata per rimuovere l'eccesso di liquidi e sali in pazienti con ritenzione idrica, resistenti ad alte dosi di diuretici, dimostrandosi in grado di correggere l'ipervolemia e rimuovere gli edemi; tuttavia il suo uso routinario non è raccomandato, ma è da riservare solo a casi altamente selezionati in cui sia risultata fallimentare la strategia diuretica convenzionale. ${ }^{46}$

\section{Bibliografia}

1. Piotr Ponikowski, Adriaan A Voors, Stefan D Anker. 2016 ESC Guidelines for the diagnosis and treatment of acute and chronic heart failure: The Task Force for the diagnosis and treatment of acute and chronic heart failure of the European Society of Cardiology (ESC) Developed with the special contribution of the Heart Failure Association (HFA) of the ESC; European Heart Journal, Volume 37, Issue 27, 14 July 2016, Pages 2129-2200.

2. Piepoli M, Binno S., Villani G.Q et al “ Management of oral chronic pharmacotherapy in patients hospitalized for acute decompensated heart failure" Intern J Card 176 (2014) 321-326.

3 Giovanni Corrao, Aldo Pietro Maggioni. Inquadramento epidemiologico dello scompenso cardiaco. G Ital Cardiol 2014;15(2 Suppl 2):10S-15S

4 Giumelli C, Reverzani A, Volpi R., Chesi G. Heart failure in real world. Italian Journal of Medicine 2015; volume 9:356-361.

5 SOLVD Investigators. Effect of enelapril on survival in patients with reduced left ventricular ejection fraction and congestive heart failure. N Engl J Med 1991; 325: 293-302.

6 Pitt B, Zannad F, Villem J.R. et al . " The effect of spironolactone on morbidity and mortality in patients with severe heart failure", NEng J Med 1999; 341:11-21.

7 Di Lenarda F, Colicicchi F, Gabrielli D. L'ospedalizzazione per scompenso cardiaco acuto: come un evento critico può diventare un'opportunità clinica. Il Pensiero Scientifico Editore. 2019, 18:15:32.

8 Lieke Bosch, Patricia Assmann, Wim J. C. de Grauw, Bianca W. M. Schalk and Marion C. J. Biermans. Heart failure in primary care: prevalence related to age and comorbidity. Primary Health Care Research \& Development cambridge.org/phc 2019).

9 Di Lenarda A., Scherillo M., Maggioni A.P. Current presentation and management of heart failure in cardiology and internal medicine hospital units: a tale of two worlds - the TEMISTOCLE study. American Heart Journal Volume 146, Issue 4, October 2003, Page 735).

10. Area Scompenso ANMCO, Firenze. Management e qualità Scompenso cardiaco: pazienti critici. G Ital Cardiol 2007; 8 (9): 568-573; P. Biagi, G. Gussoni, I. Iori et al. CONFINE Study Group. Clinical profile and predictors of in-hospital outcome in patients with heart failure: The FADOI "CONFINE" Study. International Journal of Cardiology 152 (2011), 88-94.

11. Fondazione GIMBE. Linee guida per la diagnosi e la 
terapia dello scompenso cardiaco cronico negli adulti. Evidence. Aprile 2019, Volume 11, Issue 4, e1000196; The Task Force for the diagnosis and treatment of acute and chronic heart failure of the European Society of Cardiology (ESC). 2016 ESC Guidelines for the diagnosis and treatment of acute and chronic heart failure. European Heart Journal (2016) 37, 2129-2200).

12. Fabbri G, Gorini M., Maggioni A.P. Oliva F. Area Scompenso ANMCO, Firenze. Management e qualità Scompenso cardiaco: pazienti critici. Il Pensiero Scientifico Editore. 30 set 2020,19:48:21

13. Hollenberg SM, Warner Stevenson L, Ahmad T, et al. For the American College of Cardiology Solution Set Oversight Committee. 2019 ACC Expert Consensus Decision Pathway on Risk Assessment, Management, anc Clinical Trajectory of Patients Hospitalized With Heart Failure. Journal of American College of Cardiology. 2019.08.001.

14. Chamerlain A. M., St. Sauver J.L., Gerber Y. et al. Multimorbidity in heart failure: a community perspective. The America Journal of Medicine (2015) 128,38-45.

15. Lawson C.A., Solis-Trapala I., Dahlstrom U. et al. Comorbidity health pathways in heart failure patients: a sequences of regression analysis using cross-sectional data from 10,575 patients in the swedish heart failure regisrty. Plos Medicin https://doi.org/10.1371/journal. pmed.1002540 2018.

16. Meireles M.A., Goncalves J., Neves J. Acute heart failure comorbidome: the impact of everithing else. Acta Med Port 2020 Feb;33(2):109-115.

17. Bosh L., Assmann P., De Grauw W.J.C. et al. Heart failure in primary care: prevalence related to age and comorbidity. Primary Health Care Research \& Development. Cambridge University Perss 2019.

18. Streng K.W., Nauta J.F., Hillege H.L. et al. Non cardiac comorbidities in heart failure with reduced, mid-range and preserved ejection fraction. International Journal of Cardiology 271 (2018) 132-139.

19. Triposkidias F., , Giamouzis G., Parissis J. Et al. Reframing the association and significance of comorbidities in heart failure. European Journal of Heart Failure (2016) 18, 744-758.

20. Monte S., Moscogiuri R., Germinario C. et al. Le popolazioni complesse per comorbilità. Il caso dello scompenso cardiaco. Giornale Italiano di Farmacia clinica, 21,1, 2017

21. Faris RF, Flather M et Al " Diuretics for heart failure" Cochrane database Syst Rev 2012; 2: CD 003838.

22. G. Michael Felker, Kerry L.Lee et Al "Diuretic Strategies in Patients with Acute Decompensated heart failure" N Engl J Med 2011; 364.

23. Jacob C. Jentzer, Tracy A. DeWald et A1. " Combination of loop diuretics with thiazyde type diuretics in heart failure" J Am Coll Cardiol 2010 ( 56): 1527-1534.

24. Dettli, Spring " Therapy with combinations of diuretic agents: comparative studies" Ann N Y Acad Scie 1966; 139: 471-80

25. Channer KS, McLean et Al "Combination diuretic treatment severe heart failure: a randomised controlled trial" Br Heart J 1994;71: 146-150.

26. Friedland JS et Al " Oral metolazone plus furosemide for home therapy in patients with refractory hearty failure" Lancet 1989; 333: 727-8.
27. Cooper et al. "Diuretics and risk of arrhythmic death in patients with left ventricular dysfunction" Circulation 1999;100 (12): 1311-1315).

28. McMurray JJ, Packer M, Desai AS, Gong J, Lefkowitz MP, Rizkala AR, Rouleau JL, Shi VC, Solomon SD, Swedberg K, Zile MR; PARADIGM-HF Investigators and Committees. Angiotensin-neprilysin inhibition versus enalapril in heart failure. N Engl J Med 2014;371: 993-1004.

29. Watcher R, Senni M, Belohlavek J, et a; TRANSITION Investigators. Iniziation of sacubitril/valsartan in haemodinamically stabilised heart failure patients in hospital or early after discharge; primary results of the randomised TRANSITION study. Eur J Heart Fail 2019; 21:998-1007.

30. Sauer AJ, Cole R, Jensen BC, Pal J, Sharma N, Yehya A, Vader J. Practical guidance on the use of sacubitril/valsartan for heart failure. Heart Fail Rev. 2019 Mar;24(2):167-176.

31. Velazquez EJ, Morrow DA, DeVore AD, et al; PIONEER-HF Investigators. Angiotensin-neprilysin inhibition in acute decompensated heart failure. N Engl J Med 2019; 380:539-48.

32. Van Veldhuisen DJ, Cohen-Solal A, Böhm M, Anker SD, Babalis D, Roughton M, Coats AJ, Poole-Wilson PA, Flather MD, SENIORS Investigators Beta-blockade with nebivolol in elderly heart failure patients with impaired and preserved left ventricular ejection fraction: data from SENIORS (Study of Effects of Nebivolol Intervention on Outcomes and Rehospitalization in Seniors With Heart Failure) J Am Coll Cardiol. 2009; 53:2150-8

33. Zanchetti A. Clinical pharmacodynamics of nebivolol: new evidence of nitric-oxide mediated vasodilating activity and peculiar haemodynamic properties in hypertensive patients. Blood Press. 2004;13:18-33.

34. Paulus WJ, Shah AM. NO and cardiac diastolic function. Cardiovascular Research 1999;43:595-606.

35. Coats A., Jain S. Protective effects of nebivolol from oxidative stress to prevent hypertension-related target organ damage Journal of Human Hypertension (2017) 31, 376-381.

36. Çaglar N., Dincer I. Comparison between nebivolol and ramipril in patients with hypertension and left ventricular hypertrophy: a randomized open blinded end-point (PROBE) trial. European Review for Medical and Pharmacological Sciences. 2011; 15: 1359-1368.

37. Zinman B, Wanner C, Lachin JM, et al. Empagliflozin, cardiovascular outcomes, and mortality in type 2 diabetes. N Engl J Med 2015;373:2117-28.

38. Perkovic V, Jardine MJ, Neal B, et al. Canagliflozin and renal outcomes in type 2 diabetes and nephropathy. $\mathrm{N}$ Engl J Med 2019;380:2295-306.

39. Inzucchi SE, Zinman B, Fitchett D, et al. How does empagliflozin reduce cardiovascular mortality? Insights from a mediation analysis of the EMPA-REG OUTCOME trial. Diabetes Care 2018; 41: 356-63.

40. ESC/EACTS Guidelines for the management of valvular heart disease. European Heart Journal (2017); 38 2750-2766

41. ESC Guidelines for the management of acute myocardial infarction in patients presenting with ST-segment 
elevation. European Heart Journal (2018) 39; 131-134]

42. ESC Guidelines on cardiac pacing and cardiac resynchronization therapy. European Heart Journal 2013;34;22852299]

43. ESC Guidelines for the diagnosis and treatment of acute and chronic heart failure. European Journal of Heart Failure 2016;26-27].

44. Sohaib SM, Finegold JA, Nijjer SS, et al. Opportunity to increase life span in narrow QRS cardiac resynchronization therapy recipients by deactivating ventricular pacing: evidence from randomized controlled trials. JACC Heart Fail. 2015;3:327-336]

45. Cleland JG, Abraham WT, Linde C, et al. An individual patient meta-analysis of five randomized trials assessing the effects of cardiac resynchronization therapy on morbidity and mortality in patients with symptomatic heart failure. Eur Heart J. 2013;34:3547-3556].

46. ESC Guidelines for the diagnosis and treatment of acute and chronic heart failure. European Journal of Heart Failure. 2016;28-29]. 


\title{
I peptidi natriuretici nella diagnosi e nella stratificazione del rischio nei pazienti con scompenso cardiaco
}

\author{
Andrea Montagnani, ${ }^{1}$ Lorenzo Luschi, ${ }^{2}$ Filippo Pirrotta, ${ }^{2}$ Alberto Palazzuoli ${ }^{2}$ \\ ${ }^{1}$ UOC di Medicina Interna di Pitigliano, USL Toscana Sud Est; ${ }^{2}$ UOSA Malattie Cardiovascolari, Dipartimento di Scienze \\ Mediche, Ospedale Le Scotte, Siena, Italia
}

\section{Cenni di fisiologia dei peptidi natriuretici}

I peptidi natriuretici (NPs) sono sintetizzati rispettivamente in ordine decrescente dal ventricolo $\mathrm{sx}$, ventricolo $\mathrm{dx}$ e dagli atri. Il BNP è un peptide biologicamente attivo di 32 amminoacidi, mentre l'NT-proBNP non è attivo ed è costituito da 76 amminoacidi, sono entrambi rilasciati nel sangue dal miocardio in seguito ad uno stress dei miocardiociti. ${ }^{1}$ Vengono sintetizzati in vescicole così che nella prima ora dall' insorgenza dei sintomi possono risultare nei limiti. Una volta rilasciati nel sangue il BNP è l'unica molecola biologicamente attiva ed espleta la sua funzione sui reni e sui vasi promuovendo la vasodilatazione e la natriuresi. La clearance del BNP è effettuata dal recettore del peptide $\mathrm{C}$ e dalla sua passiva escrezione attraverso i reni e gli organi con elevato flusso di sangue. L'emivita del BNP è circa 20 minuti mentre quella dell'NT-proBNP eliminato solamente dal rene è di circa 70 minuti. I peptidi natriuretici sono rilasciati nel sangue per un incremento dello stress di parete del cuore ma il loro livello basale può essere aumentato da alcune condizioni e comorbidità come l'età, il diabete, l'ipertensione, ipertensione polmonare, e l'insufficienza renale e lo stato anemico. Viceversa, l'obesità, la razza nera e l'ipertiroidismo, sono condizioni in cui i livelli degli ormoni tendono a ridursi a causa rispettivamente di una ridotta espressione recettoriale e di un aumentato metabolismo.

Corrispondente: Andrea Montagnani, UOC di Medicina Interna di Pitigliano, USL Toscana Sud Est, Italia.

E-mail: montagnaniand@gmail.com

Articolo pubblicato secondo la Creative Commons Attribution NonCommercial 4.0 License (CC BY-NC 4.0).

${ }^{\circ}$ Copyright: the Author(s), 2021

Licensee PAGEPress, Italy

QUADERNI - Italian Journal of Medicine 2021; 9(2):e7

\section{I peptidi natriuretici nella diagnosi dello scompenso cardiaco}

I NPs sono importanti biomarkers nella valutazione dello scompenso cardiaco (SC) di nuova insorgenza, ma una monitorizzazione seriata è utile anche nei soggetti affetti da SC cronico al fine di identificare eventuali riacutizzazioni. Nei Dipartimenti di Medicina Interna e di Emergenza-Urgenza nei pazienti con dispnea ingravescente il dosaggio dei peptidi è fondamentale al fine di promuovere una diagnosi differenziale e dovrebbe sempre essere associato ad una valutazione multimodale con esame obbiettivo, valutazione ecocardiografica, ultrasonografia toracica. Il valore dell'NT-proBNP come cut off nella diagnosi di scompenso cardiaco acuto a frazione di eiezione ridotta che è stato proposto è di $450 \mathrm{pg} / \mathrm{mL}, 900 \mathrm{pg} / \mathrm{mL}, 1800 \mathrm{pg} / \mathrm{mL}$ rispettivamente per i pazienti con età < a 50 aa, tra 50 e 75 e $>$ di 75 aa, i dosaggi di questi ormoni andrebbero, quindi, effettuati soprattutto al fine di escludere una malattia organica cardiaca, in quanto il loro valore predittivo negativo risulta molto elevato. Nei pazienti con filtrato renale $<$ a $30 \mathrm{~mL} / \mathrm{min}$ il BNP è più preciso e meno influenzato rispetto al NTproBNP dal ridotto filtrato renale, mentre nei pazienti che assumono gli inibitori della neprilisina l'utilizzo del BNP trova meno consenso in quanto il farmaco interferisce con la rimozione del peptide dal sangue, favorendo il suo effetto diuretico e vasodilatatore. ${ }^{2} \mathrm{Un}$ recente position paper dall'European Society of Cardiology ${ }^{3}$ ha proposto uno score diagnostico per lo $\mathrm{SC}$ a frazione di eiezione preservata, ormai rappresentativo di una quota importante dei ricoveri per SC nei reparti di medicina. Tale score includeva anche il dosaggio dei NPs. I livelli dei NPs nel paziente con setting acuto sono inferiori nei pazienti con SC a frazione d'eiezione preservata (HFpEF) rispetto a quelli con funzione sistolica ridotta (HFrEF) ma sono comunque più alti rispetto ai controlli. Questo non è solamente dovuto ad un aumento delle pressioni all'interno del ventricolo sx ma anche alla 
presenza molto frequente nei pazienti con HFrEF di comorbidità fra cui il diabete, l'ipertensione arteriosa, la malattia renale cronica ed altre che contribuiscono ad aumentare i livelli di questi biomarkers nel sangue dei pazienti. Attualmente i valori di cutoff proposti nel setting acuto dei pazienti con HFpEF sono rispettivamente di $220 \mathrm{pg} / \mathrm{ml}$ per l'NT-proBNP e di 80 $\mathrm{pg} / \mathrm{ml}$ per il BNP nei pazienti in ritmo sinusale di 660 $\mathrm{pg} / \mathrm{mL}$ e $240 \mathrm{pg} / \mathrm{mL}$ in quelli con fibrillazione atriale. I NPs non danno solamente un contributo diagnostico ma rappresentano anche indicatori prognostici di mortalità ad un anno, allo stesso modo una riduzione del NT-proBNP dall'ingresso alla dimissione inferiore al $30 \%$ è stata correlata ad una maggiore probabilità di re-ospedalizzazione e morte. ${ }^{2}$ Nei pazienti con SC cronico il dosaggio del BNP è importante per la stratificazione del rischio, in quanto livelli elevati si associano ad una maggiore incidenza di episodi ricorrenti di scompenso o di morte improvvisa. I dati pubblicati da Metra et al. hanno dimostrato come la diminuzione dei valori di NT-proBNP $24 \mathrm{~h}$ dopo l'inizio della terapia predica l'outcome a 184 giorni nello SC acuto, a sottolineare il possibile ruolo aggiuntivo del dosaggio ormonale nel definire e/o modificare l'iter terapeutico. ${ }^{4}$

\section{Relazione fra parametri ecocardiografici e livelli sierici dei peptidi natriuretici}

La diagnosi di SC oggi deve essere basata su un' integrazione che preveda il dosaggio sierico dei marcatori biochimici come i NPs, ed alcuni parametri ecografici, al fine di migliorare l'accuratezza diagnostica di questa patologia. ${ }^{6}$ Lo SC prevede che ci si possa trovare di fronte alla presenza di segni di congestione periferica (quelli che possono essere più facilmente valutati all' esame obbiettivo) e/o segni di congestione centrale a sua volta divisa in una congestione polmonare e una cardiaca anche detta congestione emodinamica. Proprio su quest' ultima si sta focolizzando l'attenzione negli ultimi anni, grazie soprattutto alla diffusione sempre maggiore degli ecocardiografi nei reparti di medicina interna e medicina d'urgenza. L'aumento delle pressioni di riempimento e in particolare l'aumento della pressione nei capillari polmonari oltre i $15 \mathrm{mmhg}$ v.n rappresentano il cardine nella diagnosi di SC. Tali parametri emodinamici possono essere valutati attraverso alcuni parametri ecocardiografici che indicano una disfunzione diastolica del ventricolo sx, e quindi un aumento della pressione telediastolica che meglio riflette l'aumento della pressione a monte dell'atrio sx. Il pattern doppler diastolico permette una valutazione di tutte e tre le fasi della diastole ovvero della fase di riempimento rapido che corrisponde all'onda $\mathrm{E}$ al doppler pulsato, la diastasi in cui la pressione tra l' atrio e il ventricolo tende ad equipararsi e che corrisponde alla distanza tra l'onda E e l'onda a, infine permette di valutare quello che è il contributo atriale al riempimento del ventricolo ovvero la presistole che è rappresentata dall'onda a al doppler pulsato. In linea generale nella disfunzione diastolica del ventricolo le pressioni iniziano prima ad aumentare nel ventricolo e solo successivamente con il progredire della malattia nell'atrio sx. La valutazione della funzione diastolica è multiparametrica e deve tenere conto non solo del rapporto $\mathrm{E} / \mathrm{a}$ ma anche di altri parametri come il tempo di decelerazione dell'onda E, il rapporto E/e', il volume atriale sx indicizzato per la massa corporea e la velocità di rigurgito tricuspidalico. In particolare, alcuni valori come un pattern doppler di tipo pseudonormale o restrittivo, un DT dell'onda $\mathrm{E}<130 \mathrm{~ms}$, un volume atriale $\mathrm{sx}>34 \mathrm{~mL} / \mathrm{m}^{2}$ e una velocità di rigurgito tricuspidalica maggiore di $2,8 \mathrm{~m} / \mathrm{s}$ sono fortemente indicativi soprattutto quando presenti almeno tre contemporaneamente di un aumento della pressione telediastolica del ventricolo sx e quindi nei capillari polmonari.

Throughton et al. hanno valutato le variazioni dei livelli sierici dei NPs nei pazienti stabili con disfunzione sistolica ventricolare in correlazione ad alcuni parametri ecocardiografici fra cui il grado di disfunzione diastolica, il grado di disfunzione ventricolare $\mathrm{dx}$ e la gravità del rigurgito mitralico. I livelli sierici dei NPs aumentavano significativamente in accordo con il grado di disfunzione diastolica. Inoltre i valori di BNP erano strettamente correlati con alcuni indici di disfunzione diastolica come il DT, E/e'e la velocità del flusso transmitralico $\mathrm{E}$, tutti parametri che riflettono un incremento delle pressioni di riempimento. Anche la disfunzione ventricolare destra e il rigurgito mitralico correlavano con i livelli sierici del BNP. ${ }^{6}$

Questo studio è stato uno dei più importanti nel valutare i livelli sieri del BNP e la loro relazione con i parametri ecocardiografici espressivi delle pressioni di riempimento, dimostrando che esiste una relazione significativa tra: i) i livelli sierici di BNP e il grado di disfunzione diastolica; ii) il BNP e la disfunzione ventricolare $\mathrm{dx}$

Infine, è stato dimostrato che a livelli sierici più bassi di BNP corrispondono un minor grado di disfunzione sistolica e diastolica del ventricolo sx, una minore disfunzione sistolica del ventricolo $\mathrm{dx}$ e un minor grado di rigurgito mitralico. Il tempo di decelerazione dell' onda $\mathrm{E}$ che rappresenta un indicatore della compliance del Vsx si è rilevato essere il più forte indice diastolico che in maniera indipendente predice i livelli sierici di BNP. ${ }^{6}$

Pochi studi hanno valutato la relazione tra BNP, ecocardiografia e stima invasiva delle pressioni di 
riempimento. Dokainish et al. ${ }^{7}$ hanno comparato indici doppler ecocardiografici con i livelli di BNP al fine di poter individuare quei pazienti ricoverati in terapia intensiva con una PCWP $>15 \mathrm{~mm}$. Nei pazienti con pregressa e conosciuta malattia cardiaca livelli di BNP oltre i $400 \mathrm{pg} / \mathrm{mL}$ hanno dimostrato simile sensibilità (91\%) ma una più debole specificità (51\% vs 91\%) rispetto agli indici doppler come $\mathrm{E} / \mathrm{e}$ ' per la ricerca dei pazienti con PCWP $>15 \mathrm{~mm} \mathrm{Hg}$. In quelli senza una cardiopatia di base, il BNP si è dimostrato più sensibile ( $81 \%$ vs $74 \%$ ) e specifico ( $83 \%$ vs $72 \%$ ) dei parametri ecocardiografici. Questo dimostra che nei pazienti con patologia cardiaca nota i parametri ecocardiografici sono superiori nella valutazione dell'aumento delle pressioni di riempimento rispetto al dosaggio sierico dei valori di BNP, mentre dove non c'è storia di patologia cardiaca questi ultimi sarebbero da preferire come strategia iniziale.

Elevati livelli di BNP e/o di NT-proBNP, inoltre, appaiono correlati con il grado di disfunzione diastolica, confermati da una sotto-analisi dello studio CHARM preserved. ${ }^{8}$ A parità di riduzione della frazione di eiezione, i valori di BNP appaiono progressivamente più elevati passando dal pattern da alterato rilasciamento fino al pattern restrittivo, secondo le metodiche standard messe a disposizione dall'esame eco-Doppler.

Sulla base della velocità di rigurgito tricuspidalico può essere anche stimata la pressione arteriosa sistolica dell' arteria polmonare che risulta spesso aumentata nei pazienti con scompenso cardiaco in fase acuta, sia nei HFreF che HfpeF. L'ipertensione polmonare nasce spesso come conseguenza di una malattia del cuore sx e in tale contesto si ha una progressione dello scompenso cardiaco dovuto ad un interessamento anche delle sezioni destre con disfunzione del ventricolo $d x$. Più raramente l'ipertensione polmonare può essere una condizione preesistente come in una forma idiopatica o primitiva di ipertensione polmonare ed andare secondariamente a coinvolgere la funzione cardiaca. In quest'ultimo contesto l'utilizzo del dosaggio sierico dei peptidi natriuretici ha un valore di stratificazione del rischio più che una valenza diagnostica. Il sovraccarico delle sezioni $\mathrm{dx}$ genera quindi il rilascio dei peptidi natriuretici, quindi condizioni come il cuore polmonare, embolia polmonare e ipertensione polmonare possono essere associati ad un incremento dei livelli sierici dei peptidi natriuretici quando associati ad aumento volumetrico e pressorio delle camere $\mathrm{dx} .^{9}$

La ricerca scientifica negli ultimi anni si è focalizzata molto anche sulla valutazione ecocardiografica dei segni di disfunzione ventricolare $\mathrm{dx}$ e quindi anche di conseguenza sullo scompenso cardiaco dx. ${ }^{10,11} \mathrm{~A}$ parte le differenze sia morfologiche, anatomiche e funzionali del ventricolo $\mathrm{dx}$ rispetto al sx, risulta evidente come un aumento delle pressioni a livello delle sezioni $\mathrm{dx}$ del cuore ha come ripercussione un aumento a livello della pressione venosa centrale e da lì verso le vene periferiche. In particolare, un aumento del diametro della vena cava $>$ di $20 \mathrm{~mm}$ e la scarsa collassabilità con la dinamica respiratoria sono forti indicatori di aumento della pressione atriale $\mathrm{dx}$ e quindi della pressione venosa centrale. Anche le vene sovraepatiche risentono di questo aumento della pressione a valle per cui trovare delle vene sovra-epatiche molto dilatate può essere anche questo un segno diagnostico aggiuntivo di scompenso cardiaco $\mathrm{dx}$. Un altro parametro che mostra una buona sensibilità e specificità nella diagnosi di scompenso cardiaco $\mathrm{dx}$ è la misura tramite doppler pulsato a livello delle vene sovra-epatiche delle tre onde a, X e Y. In condizioni fisiologiche il pulsato sulla vena sovraepatica mostra un onda a positiva quindi in avvicinamento alle vene sovra-epatiche, in quanto la sistole atriale $\mathrm{dx}$ determina una risalita del piano valvolare tricuspidalico, poi abbiamo un'onda negativa grande $\mathrm{X}$ dovuta alla sistole ventricolare con discesa del pianovalvolare tricuspidalico oltre che allo svuotamento di sangue dalle vene in atrio $\mathrm{dx}$ ed infine l'onda $\mathrm{Y}$ che rappresenta la diastole ventricolare e che si presenta anch'essa negativa dovuta al flusso di sangue in allontanamento dalle vene sovra-epatiche per lo svuotamento del sangue dall'atrio nel ventricolo dx. Durante lo SC dx l'onda $\mathrm{X}$ tende a ridursi fino a diventare positiva a causa dell'aumento del rigurgito tricuspidalico che determina una maggiore quota di sangue in avvicinamento verso le vene sovra-epatiche, mentre tende a diventare sempre più negativa l'onda $\mathrm{Y}$ che rappresenta la quota più importante di sangue che dall'atrio $\mathrm{dx}$ si dirige in ventricolo durante la diastole e che si allontana quindi dalle vene sovra-epatiche. Ovviamente, questo tipo di misurazione, ha maggiore attendibilità se accompagnato a segni di disfunzione ventricolare $\mathrm{dx}$ come una TAPSE $<17$ $\mathrm{mm}$, un'onda s' $<10 \mathrm{~cm} / \mathrm{s}$ oppure $\mathrm{RVFAC}<$ a $35 \%$, questi ultimi ad oggi rappresentano i tre parametri eco-cardiografici più utilizzati al fine di valutare la performance del ventricolo $\mathrm{dx}$, che possono essere presenti in maniera isolata oppure accompagnarsi ad un'evoluzione progressiva di una già preesistente disfunzione ventricolare sx. Purtroppo, la valutazione del doppler pulsato sulle vene sovraepatiche offre delle difficoltà tecniche non trascurabili legate innanzitutto alla finestra acustica del paziente e poi durante la dinamica respiratoria le vene sovra-epatiche tendono a scomparire a comparire sulla finestra ecografica quindi possono dare talvolta dei risultati artefattati. ${ }^{11}$ 


\section{Valore prognostico dei peptidi natriuretici nello scompenso cardiaco}

Potremmo semplicemente affermare che i NPs sono caratterizzati da un forte valore prognostico nei pazienti con SC sia in ambito ambulatoriale che in dimissione dopo un ricovero per episodio acuto di SC.

Negli ultimi anni sono molti gli studi che hanno indagato questo aspetto per i vari NPs, dal B-type natriuretic peptide (BNP), al N-terminal proBNP (NTproBNP) fino al peptide natriuretico atriale (ANP) in particolare la regione intermedia del pro-ANP (MRproANP)], che mostra caratteristiche di dosaggio più sfavorevoli (Tabella 1).

Una subanalisi dello studio Val-HeFT ha preso in considerazione 4300 pazienti ai quali erano stati effettuate misurazioni seriali di BNP al basale, a 4 e 12 mesi dall'arruolamento. I pazienti con la maggior riduzione dei valori di BNP durante il periodo di follow-up mostravano anche la più bassa mortalità $\mathrm{e}$ morbilità al contrario di quei pazienti che con i più alti livelli di BNP mostravano una mortalità più elevata. ${ }^{12}$ Il REDHOT trial valutando quasi 500 soggetti con dispnea acuta in classe NYHA II-IV con valori basali di BNP maggiori di $100 \mathrm{pg} / \mathrm{ml}$, ha trovato che i valori di BNP $>200 \mathrm{pg} / \mathrm{mL}$ erano fortemente predittivi di un outcome composto dal numero di visite per SC, ospedalizzazioni e mortalità. ${ }^{13}$ Uno studio simile, il PRIDE Trial, misurando il NT-proBNP al basale ha dimostrato una significativa capacità predittiva della mortalità ad 1 anno per i valori $>986 \mathrm{pg} / \mathrm{mL} .{ }^{14}$ Doust et al. costruendo una meta-analisi con 19 studi è arrivato a dimostrare che nei soggetti con $\mathrm{SC}$ acuto e cronico per ogni incremento di $100 \mathrm{pg} / \mathrm{mL}$ di BNP sierico corrispondeva un incremento del rischio di morte del $35 \% .{ }^{15}$

Il mancato trend negativo dei livelli di BNP durante la ospedalizzazione è ad oggi considerato un elemento prognostico negativo e come vedremo è un punto di riflessione per il miglioramento del management terapeutico del paziente con SC.

Numerosi studi, infatti, hanno valutato il valore prognostico del BNP nel trattamento dello SC acuto. Nel registro ADHERE (acute decompensated heart failure national registry) che comprendeva un database di oltre 65.000 pazienti con SC acuto l'aumento dei livelli di BNP al momento dell'ospedalizzazione per SC correlava con un incremento della mortalità intraospedaliera. ${ }^{16}$ In un altro grande registro, il Guidelines Heart Failure Registry di oltre 99.000 pazienti con SC acuto, i valori di BNP oltre la mediana si correlavano $\mathrm{ad}$ una mortalità più elevata. ${ }^{17}$ Dati simili sono stati riscontrati nel Framingham Offspring Study nel quale livelli di BNP superiori al $80^{\circ}$ percentile si associavano alla mortalità per tutte le cause ma anche allo $\mathrm{SC}$, stroke e fibrillazione atriale. ${ }^{18}$ Molti studi hanno dimostrato il ruolo prognostico verso la mortalità anche del frammento MR-proANP sia nei pazienti con SC acuto ${ }^{19}$ che cronico ${ }^{20}$ con un ruolo anche nel follow-up. ${ }^{21}$

Le più recenti linee guida ESC definiscono chiaramente il valore ed il ruolo dei NPs in particolare al momento della dimissione ospedaliera. Infatti, al momento della dimissione i pazienti con SC acuto e con valori bassi o con una riduzione particolarmente accentuata dei NPs hanno una prognosi più favorevole rispetto a coloro che mostrano invece valori alti o mancata riduzione. I valori di NPs alla dimissione dei pazienti ricoverati per SC acuto sembrano essere i migliori predittori di mortalità ad 1 anno o di riospedalizzazione rispetto alla variazione intra-ricovero o a quelli misurati all'ingresso. ${ }^{3}$

\section{Utilità di NPs nel monitoraggio della terapia}

I NPs avendo un'emivita breve risultano facilmente misurabili e correlano quantitativamente alla severità e alla prognosi dello SC. Questo aspetto ha indotto a pensare che i NPs potessero essere utili nel monitoraggio della terapia dello SC acuto e cronico. Alcuni studi hanno indagato le variazioni del BNP durante l'ospedalizzazione dei pazienti con SC acuto, dimostrando una correlazione tra BNP e la pressione capillare durante il trattamento acuto. ${ }^{22}$ Ovviamente non si suggerisce una misurazione quotidiana ma certamente un vantaggio a conoscere una serie di rilevazioni per evidenziare un trend nell'evolversi dell'episodio acuto.

Altri studi si sono focalizzati nell'analizzare la possibilità di impiegare $\mathrm{i}$ NPs nel controllare

Tabella 1. Confronto delle caratteristiche principali dei NPs nella gestione dello scompenso cardiaco.

\begin{tabular}{lccc}
\hline & MR-proANP & BNP & NT-proBNP \\
\hline Diagnosi di SC +++ & +++ & +++ & ++ \\
\hline Capacità prognostica & ++ & $+/-$ & ++ \\
\hline Variazione durante la terapia & $+/-?$ & $+/-?$ & ++ \\
\hline Evidenze per una terapia NPs-guidata & + & + & $+/-?$ \\
\hline Utilità nel pazienta ambulatoriale & + & + \\
\hline
\end{tabular}


l'efficacia terapeutica nei pazienti con SC cronico. Uno dei primi studi è stato Plasma Brain Natriuretic Peptide-Guided Therapy to Improve Outcome in Heart Failure (STARS-BNP) che ha dimostrato come un follow-up terapeutico guidato dalla valutazione dei livelli di BNP determinasse in un intervallo di 15 anni una migliore performance in mortalità e riospedalizzazioni. ${ }^{23}$ Un dato simile in un periodo di follow-up di un anno è stato poi riportato da BATTLESCARRED trial utilizzando il NT-proBNP. ${ }^{24}$ Comunque, non esiste ad oggi un consenso pieno in tal senso. Lo studio GUIDE-IT, focalizzato sulla dimostrazione dell'utilità di una terapia NPs-guidata, è stato precocemente interrotto per mancanza di risultati ${ }^{25}$ ed una recente meta-analisi ha concluso sottolineando che le evidenze per affermare definitivamente l'efficacia della terapia NP-guidata nei pazienti con SC non sono ancora sufficienti, in particolare nei soggetti con un quadro di scompenso meno grave e gestiti ambulatorialmente. ${ }^{26}$ Un ulteriore limite nella capacità predittiva dei NPs potrebbe derivare dal diffondersi dell'impiego degli inibitori della neprilisina, ovvero l'endopeptidasi che degrada i NPs. Infatti, nei pazienti che assumono sacubitril i livelli di BNP potrebbero essere maggiormente influenzati dall'inibizione della neprilisina piuttosto che dal quadro dello scompenso cardiaco riducendo quindi l'aspetto di utilità diagnostica e prognostica. ${ }^{27}$ Comunque, le più recenti linee guida ESC hanno espresso un consenso suggerendo che il beneficio della terapia NP-guidata dello scompenso cardiaco diviene più evidente quando il target dei livelli di NPs è mantenuto basso $(\mathrm{BNP}<100 \mathrm{pg} / \mathrm{mL}$, NTproBNP $<1000 \mathrm{pg} / \mathrm{mL}$ ) e quando la terapia viene modificata fino al suo raggiungimento. Con tale approccio, il dosaggio dei NPs nei pazienti con SC in follow-up non solo può avere un valore prognostico sulla mortalità e le ospedalizzazioni ma anche migliorare il management terapeutico guidando la modulazione terapeutica e motivando il paziente con la percezione quantitativa del miglioramento data dalla riduzione dei valori di NPs. ${ }^{3}$

\section{Conclusioni}

I NPs, risultando strettamente correlati agli effetti neuro-ormonali che si determinano nello SC, sono utilizzati come marcatori surrogati nella valutazione del quadro di scompenso. Le principali Linee Guida Europee ed Americane dello SC raccomandano fortemente l'impiego del BNP o NT-proBNP nella valutazione del paziente con $\mathrm{SC}$ acuto. Inoltre, esistono numerose ed importanti evidenze che i NPs abbiano un significativo valore prognostico sia al momento della dimissione del paziente con SC acuto sia nel follow-up del paziente ambulatoriale. Infine, pur esistendo un consenso di esperti sull'utilità della terapia NP-guidata in questo caso il valore del loro impiego è particolarmente migliorato se si utilizzano valori target di NPs particolarmente bassi.

In definitiva, i NPs sono ad oggi il gold-standard tra i biomarcatori nella diagnosi e prognosi dello scompenso cardiaco risultando utili nella gestione dei pazienti acuti e cronici in diversi scenari clinici.

\section{Messaggi chiave}

- L'impiego del BNP o NT-proBNP nella valutazione del paziente con dispnea ingravescente è fortemente raccomandato nei pazienti con scompenso cardiaco acuto e cronico.

- L'integrazione tra il dosaggio sierico dei peptidi natriuretici ed alcuni parametri ecocardiografici permette di migliorare l'accuratezza della diagnosi dello scompenso acuto

- I peptidi natriuretici hanno un valore prognostico sia al momento della dimissione ospedaliera del paziente con scompenso cardiaco acuto che nel follow-up del paziente ambulatoriale.

- L'impiego dei peptidi natriuretici nella gestione della terapia sembra efficace quando si utilizzano valori target particolarmente bassi.

\section{Bibliografia}

1. Richard W. Troughton, David L. Prior, J, et al. Plasma B-Type Natriuretic Peptide Levels in Systolic Heart Failure. Journal of the American College of Cardiology Vol. 43, No. 3, February 2004, Pages 416-422.

2. Sheryl L. Chow, Alan S Maisel, Inder Anand et al. Role of Biomarkers for the Prevention, Assessment, and Management of Heart Failure. Circulation. 2017; 135:e1054-e1091

3. Mueller C, McDonald K, de Boer R, et al. Heart Failure Association of the European Society of Cardiology practical guidance on the use of natriuretic peptide concentrations. European Journal of Heart Failure 2019; 21, 715-731

4. Metra M, Nodari S, Parrinello G, et al. The role of plasma biomarkers in acute heart failure. Serial changes and independent prognostic value of NT-proBNP and cardiac troponin-T. Eur J Heart Fail 2007; 9:776-86.

5. Pieske B, Tschope C, de Boer Rudolf A, et al. How to diagnose heart failure with preserved ejection fraction: the HFA-PEFF diagnostic algorithm: a consensus recommendation from the Heart Failure Association (HFA) of the European Society of Cardiology (ESC). European Heart Journal 2019;40:3297-3317.

6. Troughton RW, Prior DL, Pereira JJ, et al. Plasma Btype natriuretic peptide levels in systolic heart failure: importance of left ventricular diastolic function and right ventricular systolic function. J Am Coll Cardiol 2004;43:416-22.

7. Dokainish H, Zoghbi WA, Lakkis NM, et al. Incremental predictive power of B-type atriuretic 
peptide and tissue Doppler echocardiography in the prognosis of patients with congestive heart failure. J Am Coll Cardiol 2005;45: 223-6.

8. Dokainish H, Zoghbi WA, Lakkis NM, et al. Incremental predictive power of B-type natriuretic peptide and tissue Doppler echocardiography in the prognosis of patients with congestive heart failure. J Am Coll Cardiol 2005;45:1223-6.

9. Tromp J, Westenbrink D, Wouter Ouwerkerk A, et al. Identifying Pathophysiological Mechanisms in Heart Failure With Reduced Versus Preserved Ejection Fraction. Journal of the American college of Cardiology. 2018;72(10): 1081-1090.

10. Nagueh SF, Smiseth OA, Appleton CP, et al Waggoner. Recommendations for the Evaluation of Left Ventricular Diastolic Function by Echocardiography: An Update from the American Society of Echocardiography and the European Association of Cardiovascular Imaging. J Am Soc Echocardiogr 2016;29:277-314.

11. Lancellotti P, Price S, Edvardsen T, et al. The use of echocardiography in acute cardiovascular care: Recommendations of the European Association of Cardiovascular Imaging and the Acute Cardiovascular Care Association. European Heart Journal Cardiovascular Imaging 2015;16:119-146.

12. Anand IS, Fisher LD, Chiang YT, et al. Changes in brain natriuretic peptide and norepinephrine over time and mortality and morbidity in the Valsartan Heart Failure Trial (Val-HeFT). Circulation 2003;107:1278-83.

13. Maisel A, Hollander JE, Guss D, et al, Rapid Emergency Department Heart Failure Outpatient Trial Investigators. Primary results of the Rapid Emergency Department Heart Failure Outpatient Trial (REDHOT). A multicenter study of B-type natriuretic peptide levels, emergency department decision making, and outcomes in patients presenting with shortness of breath. J Am Coll Cardiol 2004;44:1328-33.

14. Januzzi JL Jr, Sakhuja R, O’Donoghue M, et al. Utility of amino-terminal pro-brain natriuretic peptide testing for prediction of 1-year mortality in patients with dyspnea treated in the emergency department. Arch Intern Med 2006;166: 315-20.

15. Doust JA, Pietrzak E, Dobson A, et al. How well does B-type natriuretic peptide predict death and cardiac events in patients with heart failure: systematic review. BMJ 2005;330:625

16. Fonarow GC, Peacock WF, Phillips CO, et al, ADHERE Scientific Advisory Committee and Investigators. Admission B-type natriuretic peptide levels and in- hospital mortality in acute decompensated heart failure. J Am Coll Cardiol 2007;49:1943-50.

17. Hsich EM, Grau-Sepulveda MV, Hernandez AF, et al. Relationship between sex, ejection fraction, and B-type natriuretic peptide levels in patients hospitalized with heart failure and associations with inhospital outcomes: findings from the Get with the Guideline-Heart Failure Registry. Am Heart J 2013;166:1063-71.

18. Wang TJ, Larson MG, Levy D, et al. Plasma natriuretic peptide levels and the risk of cardiovascular events and death. N Engl J Med 2004;350:655-63.

19. Shah RV, Truong QA, Gaggin HK, et al. Mid-regional proatrial natriuretic peptide and proadrenomedullin testing for the diagnostic and prognostic evaluation of patients with acute dyspnoea. Eur Heart J 2012;33:2197-205.

20. Masson S, Latini R, Carbonieri E, et al. The predictive value of stable precursor fragments of vasoactive peptides in patients with chronic heart failure: data from the GISSI-heart failure (GISSI-HF) trial. Eur J Heart Fail 2010;12:338-47.

21. Miller WL, Hartman KA, Grill DE, et al. Serial measurements of midregion proANP and copeptin in ambulatory patients with heart failure: incremental prognostic value of novel biomarkers in heart failure. Heart 2012;98:389-94.

22. Kazanegra R, Cheng V, Garcia A, et al. A rapid test for B-type natriuretic peptide correlates with falling wedge pressures in patients treated for decompensated heart failure: a pilot study. J Card Fail 2001;7:21-9.

23. Jourdain P, Jondeau G, Funck F, et al. Plasma brain natriuretic peptide-guided therapy to improve outcome in heart failure: the STARS-BNP Multicenter Study. J Am Coll Cardiol 2007;49:1733-39.

24. Lainchbury JG, Troughton RW, Strangman KM, et al. $\mathrm{N}$-terminal pro-B-type natriuretic peptide-guided treatment for chronic heart failure: results from the BATTLESCARRED (NT-proBNP-Assisted Treatment To Lessen Serial Cardiac Readmissions and Death) trial. J Am Coll Cardiol 2009;55:53-60.

25. Felker GM, Anstrom KJ, Adams KF, et al. Effect of Natriuretic Peptide-Guided Therapy on Hospitalization or Cardiovascular Mortality in High-Risk Patients With Heart Failure and Reduced Ejection Fraction: A Randomized Clinical Trial. JAMA 2017;318:713-20.

26. McLellan J, Bankhead CR, Oke JL, et al. BMJ Evidence-Based Medicine 2020;25:33-37.

27. McKie PM, Burnett JC Jr. NT-proBNP: the gold standard biomarker in heart failure. J Am Coll Cardiol 2016;68:2437-9. 


\title{
L'educazione terapeutica del paziente scompensato
}

\author{
Maria Delia Corbo, Enrica Vitale, Michele Correale, Natale Daniele Brunetti, Massimo Iacoviello
}

Scuola di Specializzazione in Malattie dell'Apparato Cardiovascolare, Dipartimento di Scienze Mediche e Chirurgiche, Università degli Studi di Foggia, Struttura Complessa di Cardiologia, AOU Policlinico Riuniti di Foggia, Italia

\section{Introduzione}

Le profonde trasformazioni che hanno interessato negli ultimi decenni la nostra società hanno favorito il verificarsi di importanti cambiamenti demografici caratterizzati dall'aumento della sopravvivenza e dal progressivo invecchiamento della popolazione, cui si è associato l'aumento di condizioni morbose a carattere cronico caratterizzate spesso da una particolare complessità di gestione. ${ }^{1}$ In tale contesto, lo scompenso cardiaco cronico (SCC) è una delle cronicità con maggiore rilevanza clinica ed impatto socio-economico, considerata la scadente qualità di vita dei pazienti ad esso associata, le frequenti ospedalizzazioni, l'elevata mortalità e complessità gestionale e terapeutica.

La risposta alle complesse esigenze assistenziali dello SCC si traduce in un programma di intervento altrettanto complesso ed articolato, basato sulla presa in carico da parte di un ambulatorio dedicato ${ }^{2}$ il cui team multidisciplinare e multiprofessionale, ha competenza specifica nel campo e garantisce una gestione clinica capace di migliorare la qualità ed aspettativa di vita dei pazienti.

Tra le attività che caratterizzano l'ambulatorio dedicato allo scompenso cardiaco, l'educazione terapeutica del paziente riveste sicuramente un ruolo di primaria importanza. Il suo obiettivo è garantire l'empowerment del paziente ed il conseguente aumento della aderenza al piano diagnostico terapeutico consigliato. Il termine empowerment è nato negli anni sessanta nel campo della psicologia di comunità, ma è stato, in seguito, utilizzato in altri ambiti definendo

Corrispondente: Massimo Iacoviello, Dipartimento di Scienze Mediche e Chirurgiche, Università degli Studi di Foggia, Via L. Pinto 1, Foggia, Italia.

E-mail: massimo.iacoviello@unifg.it

Articolo pubblicato secondo la Creative Commons Attribution NonCommercial 4.0 License (CC BY-NC 4.0).

${ }^{\circ}$ Copyright: the Author(s), 2021

Licensee PAGEPress, Italy

QUADERNI - Italian Journal of Medicine 2021; 9(2):e8 quel processo con cui le persone acquisiscono controllo sulla propria vita e sul proprio contesto ambientale, al fine di massimizzare le abilità e produrre risultati soddisfacenti. Nella gestione delle malattie croniche l'empowerment è strettamente legato all'aderenza del paziente. Quest'ultima è definita come coinvolgimento attivo e collaborativo del paziente a cui si chiede di partecipare alla pianificazione e all'attuazione del trattamento elaborando un consenso basato sull'accordo. ${ }^{3}$ Differisce dalla compliance, che è intesa come il grado in cui il comportamento di una persona (es. assunzione di farmaci, osservanza di diete, cambiamenti nello stile di vita) coincide con le raccomandazioni del medico. ${ }^{4}$

Se, dunque, l'aderenza rappresenta l'obiettivo finale utile ad un miglioramento dello stato di salute ed alla riduzione complessiva dei costi sanitari dello SCC, 5,6 l'educazione terapeutica e l'empowerment del paziente rappresentano gli strumenti principali attraverso i quali il malato non è più unicamente oggetto delle cure, ma attore principale delle stesse.

\section{Aderenza del paziente e pianificazione dell'intervento educazionale}

La scarsa aderenza dei pazienti rappresenta un problema molto diffuso, ${ }^{7}$ che determina un peggioramento della prognosi, con aumentato rischio di ospedalizzazione e morte. ${ }^{8,9}$ Nello studio CHARM, sia i pazienti del gruppo in trattamento con candesartan, sia quelli del gruppo placebo presentavano una significativa riduzione della mortalità in caso di assunzione di più dell' $80 \%$ dei farmaci prescritti. ${ }^{10}$ Nello studio TEMISTOCLE, nel 50\% dei pazienti con re-ospedalizzazione per riacutizzazione di scompenso a 3 mesi, il nuovo ricovero si verificava per la mancata aderenza alla terapia o incomprensione dei consigli terapeutici. ${ }^{11}$ L'aumento della aderenza alla terapia farmacologica nei pazienti con scompenso cardiaco riduce la durata della degenza, $i$ tassi di riospedalizzazione ed i costi delle cure. ${ }^{12}$ Un'alta percentuale di riammissioni ospedaliere è riconducibile a discrepanze nella terapia farmacologica assunta prima e dopo il ricovero. ${ }^{13,14}$

La personalizzazione di un efficace intervento edu- 
cazionale dovrebbe basarsi su due aspetti fondamentali ovvero la valutazione dei fattori capaci di condizionare l'aderenza terapeutica e la valutazione, per ciascun paziente, dell'aderenza terapeutica stessa (Figura 1).

\section{Fattori condizionanti l'aderenza}

La mancata aderenza è influenzata da svariati fattori (Tabella 1): condizioni legate al paziente; condizioni mediche; terapia; aspetti socioeconomici; organizzazione dei sistema sanitario. ${ }^{15-17}$

Nell'ambito di un intervento educazionale dovrebbe essere riservata particolare attenzione ai fattori legati al paziente. Il paziente ad alto rischio di mancata aderenza è il paziente di età avanzata, con deficit cognitivi, visivi, acustici, con comorbilità, scarsa alfabetizzazione sanitaria e scarsa consapevolezza della patologia.

Tra le patologie associate, ha una particolare rilevanza la sindrome depressiva. ${ }^{18}$ I pazienti depressi hanno maggiori probabilità di essere non aderenti rispetto ai pazienti non depressi a causa dei sentimenti di disperazione, delle scarse energie per portare avanti il regime terapeutico, dell'isolamento sociale, nonché delle possibili riduzioni delle capacità cognitive che possono influenzare la memoria. ${ }^{19}$

Tra i fattori legati al paziente, anche lo stato coniugale e la presenza di caregivers hanno un ruolo es- senziale, potendo contribuire in maniera rilevante alla gestione del paziente. Un intervento educazionale, dunque, non può prescindere dalla valutazione del contesto familiare e sociale e dal coinvolgimento dei familiari e/o caregivers (es. badanti).

Nella pianificazione dell'intervento educazionale,

Tabella 1. Fattori di non aderenza (World Health Organization).

\begin{tabular}{|c|c|}
\hline Paziente & $\begin{array}{l}\text { Percezione di mancanza di efficacia } \\
\text { Scarsa alfabetizzazione sanitaria } \\
\text { Limitazioni fisiche (vista, aspetti cognitivi) } \\
\text { Depressione e isolamento sociale } \\
\text { Decadimento cognitivo }\end{array}$ \\
\hline Condizione medica & $\begin{array}{l}\text { Alto regime di complessità dello scompenso } \\
\text { Polifarmacoterapia per multiple comorbilià }\end{array}$ \\
\hline Terapia & $\begin{array}{l}\text { Frequenza delle dosi } \\
\text { Polifarmacoterapia } \\
\text { Effetti collaterali }\end{array}$ \\
\hline $\begin{array}{l}\text { Aspetti } \\
\text { socioeconomici }\end{array}$ & $\begin{array}{l}\text { Costi } \\
\text { Difficoltà di accesso alla farmacia } \\
\text { Mancanza di supporto }\end{array}$ \\
\hline Sistema sanitario & $\begin{array}{l}\text { Scarsa comunicazione } \\
\text { Luogo di cura } \\
\text { Nessun rimborso automatico }\end{array}$ \\
\hline
\end{tabular}

\begin{tabular}{|c|c|c|c|}
\hline $\begin{array}{c}\text { Diagnosi o Ricovero per } \\
\text { Scompenso Cardiaco }\end{array}$ & Dimissione & $\begin{array}{c}\text { Controllo post- } \\
\text { dimissione }\end{array}$ & Follow-up \\
\hline
\end{tabular}
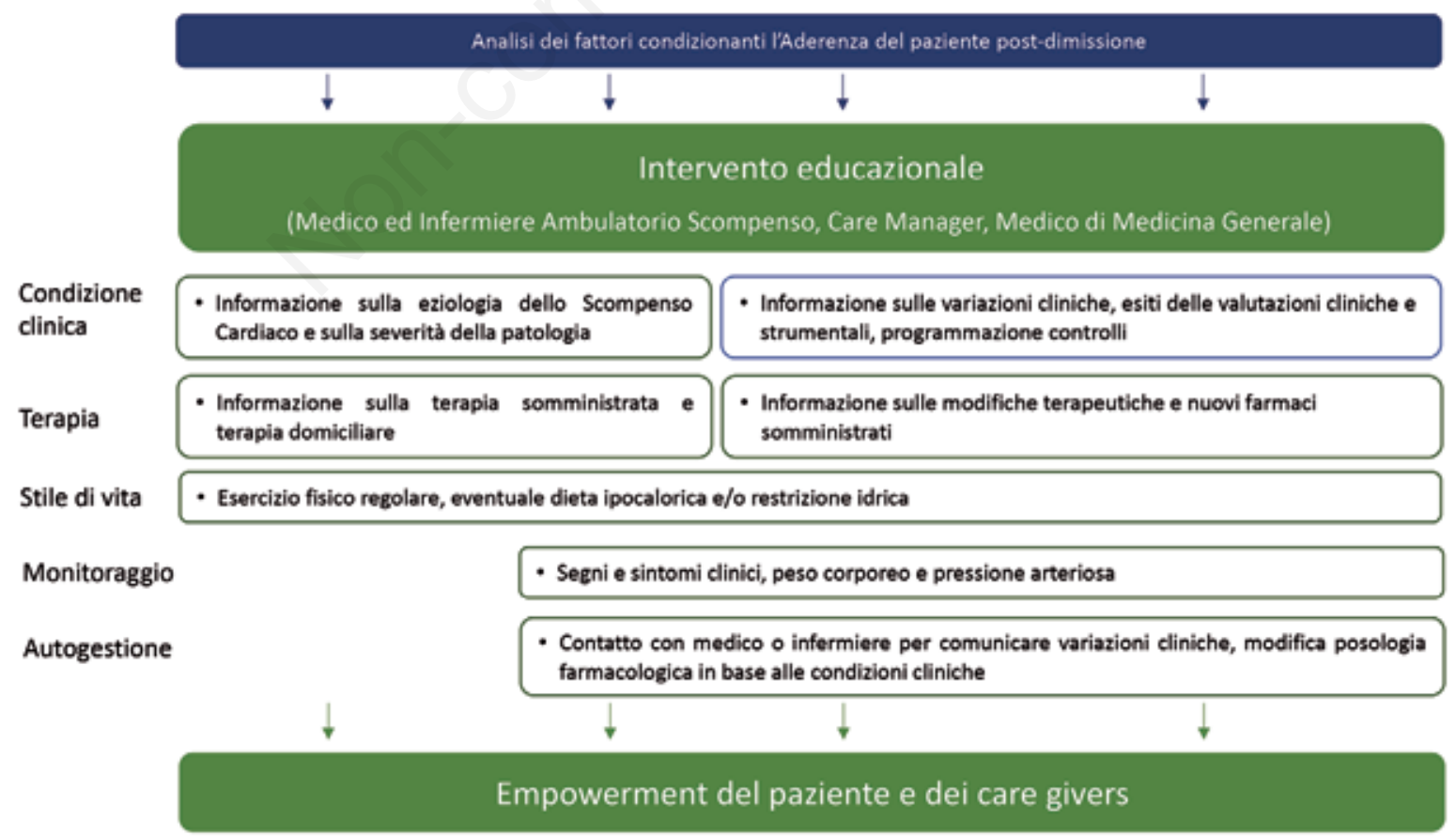

Figura 1. Sintesi dei momenti clinici e delle caratteristiche dell'intervento educazionale nel paziente affetto da scompenso cardiaco cronico. 
infine, oltre a tutti i fattori elencati in Tabella 1, una particolare considerazione dovrebbe essere riservata anche $\mathrm{i}$ fattori legati alla prescrizione farmacologica (elevata frequenza delle somministrazioni, elevato numero dei farmaci e comparsa di effetti collaterali) che possono svolgere un ruolo essenziale nel raggiungimento di un'adeguata aderenza.

\section{La valutazione dell'aderenza}

La valutazione dell'aderenza del paziente rappresenta il secondo elemento sul quale personalizzare l'intervento educazionale. I metodi di valutazione dell'aderenza includono questionari per il paziente, autovalutazioni, conteggio delle pillole, tasso delle prescrizioni, valutazione della risposta clinica del paziente, monitor elettronici dei farmaci, misurazione dei marker fisiologici e diari dei pazienti. ${ }^{15,16}$ L'ideale sarebbe misurare l'aderenza oggettivamente attraverso sistemi come il MEMS (un sistema di monitoraggio elettronico con un chip incorporato nel tappo di una confezione, che registra ogni volta che il tappo viene rimosso). Con tale sistema si è evidenziato come l'aderenza auto-riportata possa in realtà sovrastimare la vera aderenza dei pazienti. ${ }^{20}$

Ogni ambulatorio dedicato allo SCC dovrebbe, comunque, predisporre un sistema di valutazione utile a valutare l'aderenza alla terapia farmacologica consentendo sia la personalizzazione dell'intervento educazionale sia la valutazione degli esiti dello stesso.

\section{L'educazione e l'empowerment del paziente}

L'articolazione di un percorso educazionale finalizzato a raggiungere un'adeguata aderenza ha nell'empowerment del paziente e dei familiari/caregivers lo strumento principale. Il processo di empowerment dovrebbe consentire di raggiungere la piena consapevolezza della malattia da parte del paziente e dei caregivers e, conseguentemente, una piena aderenza alla terapia ed una autogestione efficace.

\section{La consapevolezza della malattia}

Il paziente con SCC ed i caregivers dovrebbero acquisire le principali nozioni riguardanti l'eziologia dello scompenso cardiaco e la possibile evoluzione della sindrome. Ovviamente, le stesse informazioni dovrebbero essere acquisite per tutte le comorbilità eventualmente presenti. Il paziente, infine, dovrebbe avere completa consapevolezza della prognosi legata alla sua situazione clinica.

\section{Il percorso diagnostico}

Il medico dovrebbe rendere edotto il paziente della pianificazione del suo percorso diagnostico-terapeutico. Il paziente dovrebbe essere informato sulle prin- cipali informazioni che vengono ottenute dalle valutazioni cliniche e strumentali consigliate ed eseguite, sul proprio piano di visite durante follow-up, sulla cadenza dei controlli ambulatoriali ma anche sul monitoraggio domiciliare di seguito riportato.

\section{Il percorso terapeutico}

Paziente e caregiver devono avere piena consapevolezza degli effetti benefici e quelli potenzialmente negativi dei farmaci e della modalità della loro assunzione..$^{21}$ Analogamente, in caso di procedure interventistiche (dall'impianto del cardiovertitore defibrillatore e/o resincronizzazione ventricolare sino a giungere alle procedure di emodinamica interventistica e terapia sostitutiva cardiaca) il paziente deve essere messo a conoscenza dei potenziali benefici e dei rischi legati alle procedure.

\section{L'auto monitoraggio}

Un aspetto fondamentale dell'empowerment è la capacità del paziente di eseguire il monitoraggio dei propri segni e sintomi nonché di parametri strumentali come pressione arteriosa, peso, saturazione arteriosa, monitoraggio del cardiovertitore defibrillatore $\mathrm{o}$ altro. Il paziente dovrebbe acquisire tutti gli strumenti utili per gestire la malattia in maniera autonoma, in modo da sentirsi più competente e sereno nell'affrontare la propria patologia, con un beneficio anche in termini di qualità di vita. I pazienti dovrebbero imparare ad individuare i segni precoci di accumulo dei liquidi (aumento del peso corporeo e degli edemi declivi, accentuazione dei sintomi quali dispnea e astenia), i segni precoci di bassa gittata, ischemia o aritmie (ipotensione, angina, cardiopalmo, sincope, DC-shock).

Strumenti di supporto per il paziente nel processo di autocura includono l'uso indipendente di strumenti di misurazione, stesura di un diario dei parametri monitorati, schemi di adeguamento posologico della terapia diuretica, l'utilizzo di portapillole, feedback sui sintomi clinici tramite messaggi, telefonate o e-mail. ${ }^{22}$

\section{La capacità di autogestione}

Sempre di più, nel corso delle ultime decadi, si è passati, per il paziente con cronicità, da un approccio puramente farmacologico di trattamento dei sintomi al 'self-care' (letteralmente cura di sé). Per self care ci si riferisce a un processo di scelte che conduce al mantenimento della cura di se stessi, alla percezione del sintomo e alla gestione del sintomo. Il self-care è parte integrante del processo di empowerment ed è caratterizzato dall'autogestione della terapia prescritta (in particolare la modulazione della terapia diuretica), dall'eventuale ricorso al Pronto Soccorso, dalla necessità di prendere contatto con il Medico di Medicina Generale e l'Ambulatorio dello Scompenso Cardiaco. 


\section{Il percorso educazionale}

Per raggiungere l'empowerment è necessario assicurare un adeguato intervento di educazione terapeutica coinvolgendo il paziente in interventi di comunicazione, di educazione, di informazione e di motivazione al cambiamento. Tuttavia, spesso gli interventi educativi sono incompleti e impartiti frettolosamente al momento della consegna della lettera di dimissione. Ciò comporta delle conseguenze sul grado di aderenza alle terapie prescritte e alle raccomandazioni suggerite, poco seguite già a breve distanza di tempo dalla dimissione, con ricadute importanti in termini di prognosi. ${ }^{21}$ Counseling, coaching, corretta scelta delle strategie educazionali ed approccio multiprofessionale rappresentano, in questo contesto, elementi indispensabili per il successo dell' intervento educazionale.

\section{Il counseling}

Secondo la definizione dell'OMS, l'educazione terapeutica si configura come un aiuto ai pazienti per acquisire e mantenere competenze rispetto alla loro malattia cronica e un processo continuo che comprende informazione, consapevolezza, apprendimento, supporto psico-sociale e aderenza alla terapia. ${ }^{23} \mathrm{Il}$ counseling, attraverso il colloquio, mira a rafforzare la motivazione del paziente nel raggiungimento di obiettivi condivisi e realistici. Il counseling è inteso come attività che orienta, supporta e sviluppa le potenzialità del paziente, promuovendone atteggiamenti attivi e capacità di scelta. Lo scopo è quello di consentire ai pazienti e ai familiari la comprensione della patologia, del trattamento e delle responsabilità per una cooperazione efficace con i professionisti volta a migliorare la propria qualità di vita.

È un processo dinamico che deve tener conto del corso della malattia e dei bisogni del malato, richiede un continuo adattamento da parte del paziente e coinvolge la sua vita quotidiana e il suo ambiente psicosociale, la famiglia e gli amici.

\section{Il coaching}

Qualsiasi sia l'approccio che si scelga, l'obiettivo finale è quello di rendere il paziente protagonista e indipendente nel prendersi cura di sé stesso nella gestione della sua malattia, facendogli acquisire un maggior senso di controllo ed una maggiore motivazione alla cura. Il coaching si pone come obiettivo il passaggio da una condizione passiva del paziente nei confronti della malattia ad una condizione attiva, consapevole del proprio stato, con lo scopo di imparare a gestirlo.

\section{Gli approcci educazionali}

Diversi sono gli approcci educazionali che sono stati testati nei vari studi. Tra questi, incontri e sessioni educative e di training, materiale informativo e formazione 'web based'. Gli interventi dovrebbero essere differenziati nei diversi gruppi di pazienti, in base all'età, alla cultura e/o alla geografia. Queste differenze devono essere attentamente valutate affinché l'intervento medico possa essere costruito sul paziente singolo per massimizzarne l'efficacia. ${ }^{21}$ Le sessioni educazionali pianificate possono essere integrate da brochure, newsletter, programmi per computer e materiale interattivo. ${ }^{21}$

Alcune strategie specifiche possono contribuire al successo del processo educazionale, dal 'discharge contract', anche con l'ausilio di materiale illustrato, e al metodo 'teach back' (la persona racconta con parole proprie quanto è stato appena spiegato) volto a verificare che il paziente abbia realmente compreso quanto esposto. ${ }^{24,25}$ Può succedere, infatti, che i pazienti rispondano alle istruzioni mediche con una serie di cenni e semplici 'sì', senza però avere compreso realmente le indicazioni che vengono date loro. È quindi importante chiedere al paziente di spiegare il motivo per cui viene prescritto ciascun farmaco o il senso di un determinato comportamento da seguire, perché potrebbe rivelare la loro mancanza di comprensione, preannunciando un'elevata probabilità di scarsa aderenza.

\section{Il ruolo del medico e dell'infermiere professionale}

Nell'ambito della educazione terapeutica sicuramente il medico dell'ambulatorio scompenso ed il medico di medicina generale svolgono un ruolo essenziale. Ad ogni valutazione ambulatoriale i medici dovrebbero sia accertare il grado di aderenza sia proseguire ed adattare il percorso educazionale di empowerment del paziente alle condizioni cliniche rilevate. È importante sottolineare in questo contesto l'enorme rilevanza della figura dell'infermiere dedicato all'ambulatorio scompenso nel percorso educazionale. Il successo dei team specializzati interprofessionali nell'ottenere una maggiore aderenza al trattamento dello SCC è stato ampiamente dimostrato, ${ }^{26}$ così come sono dimostrati i risultati positivi di un counseling affidato a figure infermieristiche. ${ }^{27}$ L'infermiere (sia a livello ambulatoriale sia ospedaliero e domiciliare ${ }^{28}$ ) assume il ruolo di 'facilitatore', favorendo il confronto con il paziente, incoraggiando la motivazione e la responsabilità individuale del malato e della sua famiglia. ${ }^{28} \mathrm{Si}$ occupa dei bisogni clinico-socio-assistenziali della persona tenendo conto del suo nucleo familiare, del monitoraggio clinico, strumentale e bioumorale, con controlli ambulatoriali o domiciliari o con follow-up telefonici, la cui frequenza è stabilita da una programmazione condivisa con il medico di riferimento. ${ }^{2,29}$ Garantisce la continuità delle cure e l'integrazione multidisciplinare. Si configura come il punto di incontro tra il malato e le figure mediche (medico di medicina generale, medico 
specialista e strutture territoriali e ospedaliere) con competenze tecnico-professionali, di carattere organizzativo, metodologico e relazionale. ${ }^{30,31}$ In alcuni percorsi assistenziali di cronicità, inoltre, si è introdotta una figura infermieristica di raccordo tra ospedale e territorio, il care manager, che contribuisce, oltre che al percorso assistenziale, al percorso educazionale dei pazienti.

In questo modo può direttamente verificare l'ambiente in cui vive il malato, valutare se il programma di self-management è applicato correttamente (dieta, introito di liquidi e sale, controllo del peso, gestione della terapia) e correggere eventuali incongruità consentendo la rimodulazione del piano di cura 'sul campo' ${ }^{29}$

\section{Il ruolo della telemedicina nell'educazione terapeutica}

Negli ultimi anni è emersa la possibile rilevanza della telemedicina nell'educazione sanitaria. I presidi legati alla telemedicina, infatti, non sono solo rappresentati da promemoria telefonici, device come bilance, ossimetri e monitor della pressione sanguigna ${ }^{12} \mathrm{ma}$ anche da sistemi che possono rinforzare l'educazione sanitaria. È stato dimostrato che questo tipo di interventi, comprese le videoconferenze domiciliari e la comunicazione telefonica, riducono i ricoveri ospedalieri ed i costi delle cure nello SCC. ${ }^{32,33}$ Anche il semplice intervento telefonico ha dimostrato di migliorare l'aderenza al farmaco. ${ }^{34}$

Si sta, inoltre, progressivamente affermando l'utilizzo di applicazioni e device elettronici che aiutano il paziente e il medico nella gestione domiciliare dello scompenso cardiaco. Questi strumenti sono rappresentati da applicazioni, generalmente installabili su smartphone, che possono memorizzare dati quali peso, pressione, frequenza cardiaca, saturazione di ossigeno ma anche riportare esami di laboratorio, esami strumentali, visite ambulatoriali e ricoveri, costruendo una vera storia clinica del paziente. Queste stesse applicazioni permettono il controllo e la verifica della terapia farmacologica, possono impostare dei promemoria per gli appuntamenti di follow-up e presentare materiale informativo utile a proseguire il processo educazionale.

La gestione in telemedicina dei pazienti con scompenso cardiaco può portare a miglioramenti nei tassi di riammissione correlati allo scompenso cardiaco, ${ }^{35,36}$ anche se sono necessari ulteriori dati per verificarne il rapporto costo-efficacia. ${ }^{37}$

\section{I momenti dell'educazione terapeutica: dal ricovero al post-dimissione}

Il percorso di educazione terapeutica si svolge nei vari momenti assistenziali del paziente affetto da SCC. Il momento del ricovero o diagnosi di scompenso car- diaco rappresenta, spesso, il punto iniziale del percorso educazionale, che prosegue lungo l'intero follow-up del paziente.

\section{Il ricovero}

Il ricovero ospedaliero può essere un momento rilevante nel percorso educazionale del paziente. Durante il ricovero i pazienti possono, ad esempio, essere informati sulle valutazioni cliniche e strumentali eseguite e sulla risposta alle cure, possono essere istruiti sui farmaci che dovrebbero assumere (ad esempio mentre gli infermieri passano per dare ai pazienti i farmaci ne sottolineano l'importanza di assumerli, cosa che spesso non si verifica durante le visite ambulatoriali). ${ }^{38}$

Il ricovero, d'altro canto, è di per sé un momento di disorientamento sia per il paziente che per la famiglia e il ritorno a domicilio rappresenta un momento cruciale..$^{39}$ L'errore in cui si può incorrere è di non instaurare un vero e proprio colloquio con il malato e la sua famiglia, ma limitarsi a comunicare loro decisioni già prese, con il rischio che non vengano adeguatamente comprese, accettate e seguite. ${ }^{21}$ È invece essenziale comunicare con il paziente e renderlo consapevole dei rischi e dei benefici di un determinato approccio terapeutico, cercando di rispettare le preferenze espresse da quest'ultimo. Ed è altrettanto importante estendere, sin dal ricovero, l'intervento di educazione terapeutica al caregiver (cominciando già durante il ricovero per proseguire fino alla dimissione), che con il suo ruolo può aiutare a migliorare la prognosi. ${ }^{40}$

\section{La dimissione}

Altrettanto importante è la pianificazione completa e multidisciplinare delle dimissioni, indispensabile perché si possano ridurre $\mathrm{i}$ tassi di riospedalizzazione precoce. ${ }^{41-43} \mathrm{Al}$ momento della dimissione è importante valutare il grado di consapevolezza e di comprensione del paziente per tutti gli aspetti che interessano la sua malattia come precedentemente descritto.

\section{Il follow-up}

Alla dimissione il paziente deve essere preso in carico dal medico di medicina generale, con il quale è opportuno interfacciarsi anche durante il ricovero, e dall'ambulatorio dedicato allo SCC. Quasi la metà infatti delle riammissioni per scompenso cardiaco si verifica prima della prima visita ambulatoriale ${ }^{44,45} \mathrm{Per}$ tale motivo, deve essere programmato un follow-up stretto con un contatto telefonico entro la prima settimana ed un controllo ambulatoriale entro 15-30 giorni dalla dimissione, tenendo sempre in considerazione quelle che sono le caratteristiche culturali del paziente, il suo contesto sociale, il tipo di supporto familiare che è in grado di ricevere e la disponibilità di assistenza domiciliare. ${ }^{21}$ In occasione del controllo post-dimis- 
sione e dei controlli successivi è opportuno che il processo educazionale prosegua.

Una volta giunto a domicilio, il primo passo per garantire l'aderenza è determinare se il paziente riesce a comunicare ed elaborare le informazioni. Possono essere d'aiuto tecniche quali il monitoraggio delle prescrizioni, il conteggio delle pillole e il coinvolgimento di amici o familiari.

Attenzione va data anche ai costi complessivi della cura (ad esempio prescrivere farmaci a basso costo se di efficacia simile o mantenere basse le quote di partecipazione dei pazienti), perché spesso questi possono superare le capacità economiche del paziente, contribuendo alla scarsa aderenza terapeutica. ${ }^{46}$

Particolare rilevanza ha anche la prosecuzione dell'educazione terapeutica in occasione dei controlli ambulatoriali programmati. L'educazione terapeutica, infatti, dovrebbe essere un percorso continuo, capace di adattarsi alle variazioni nella storia clinica del paziente. È stato evidenziato che l'aderenza alla terapia diminuisce nel tempo, indipendentemente dal fatto che i pazienti siano aderenti alla prima valutazione o meno. Pertanto, è essenziale che il medico valuti l'aderenza ad ogni visita, anche quando i pazienti sembrano esserlo stati in passato e prosegua nel percorso di empowerment dei pazienti. ${ }^{46}$

I controlli post-dimissione dovrebbero prevedere la revisione terapeutica (reconciliation). È il processo di compilazione e aggiornamento della lista di tutti i farmaci che il paziente sta assumendo (riportando il nome del farmaco, la dose, le modalità di somministrazione). ${ }^{21}$ Tale lista, sempre aggiornata, comprensiva delle motivazioni dei cambiamenti effettuati, accompagna il paziente durante tutto il ricovero, con lo scopo di somministrare al paziente la terapia corretta in tutti i livelli di transizione di cura. La procedura prevede: identificare i farmaci che il paziente già assume, confrontare la vecchia terapia con la nuova (rilevando anche eventuali interazioni farmacologiche).

\section{Conclusioni}

I percorsi educazionali dei pazienti affetti da scompenso cardiaco cronico rivestono un ruolo di primaria importanza per ottimizzare la gestione dei pazienti ed aumentare l'aderenza al percorso diagnostico-terapeutico. Tutte le figure professionali coinvolte nella gestione clinica sono responsabili nell'educazione del paziente e dei familiari/caregivers, dal medico dell'ambulatorio scompenso, al medico di medicina generale, al personale infermieristico dedicato. I percorsi educazionali devono essere continui e caratterizzare i vari momenti della storia naturale dei pazienti, dal ricovero, alle valutazioni ambulatoriali alla gestione domiciliare. Il loro obiettivo è raggiungere l'empowerment del paziente e dei familiari consen- tendo di renderli pienamente partecipi e loro stessi protagonisti del percorso di cura.

\section{Bibliografia}

1. Radini D, Mennuni M, Accardo S, et al. ANMCO Position paper: From acute to chronic disease: the needs of complex cardiac patients. G Ital Cardiol (Rome). 2019;20:593-608.

2. Aspromonte N, Gulizia MM, Di Lenarda A, et al. Documento di consenso ANMCO/SIC. La rete cardiologica per la cura del malato con scompenso cardiaco: organizzazione dell'assistenza ambulatoriale. Addenda online. G Ital Cardiol 2016;17:e1-e22.

3. Meyers L B, Midence K. Adherence to treatment in medical condition. Harwood Academic Publisher, Amsterdam, NE, 1998.

4. Haynes RB. Compliance in health care. John Hopkins University Press, Baltimora, 1979.

5. Kronish IM, Ye S. Adherence to cardiovascular medications: lessons learned and future directions. Prog Cardiovasc Dis 2013; 55:590-600 .

6. Sabaté E (ed). Adherence to Long-Term Therapies: Evidence for Action. Geneva: World Health Organization, 2003.

7. Wu JR, Moser DK, Lennie TA, Burkhart PV. Medication adherence in patients who have heart failure: a review of the literature. Nurs Clin North Am 2008;43:133-53.

8. Hope CJ, Wu J, Tu W, Young J, Murray MD. Association of medication adherence, knowledge, and skills with emergency department visits by adults 50 years or older with congestive heart failure. Am J Health Syst Pharm 2004;61:2043-9.

9. Michalsen A, Konig G, Thimme W. Preventable causative factors leading to hospital admission with decompensated heart failure. Heart 1998;80:437-41.

10. Granger BB, Swedberg K, Ekman I, et al. Adherence to candesartan and placebo and outcomes in chronic heart failure in the CHARM programme: double-blind, randomised, controlled clinical trial Lancet 2005;366:2005-2011.

11. Di Lenarda A, Scherillo M, Maggioni AP et al.; Current presentation and management of heart failure in cardiology and internal medicine hospital units: a tale of two worlds--the TEMISTOCLE study. Am Heart J. 2003;146:E12.

12. Riles EM, Jain AV, Fendrick AM. Medication adherence and heart failure. Curr Cardiol Rep. 2014 Mar;16(3):458. doi: 10.1007/s11886-013-0458-z. PMID: 24464304.

13. Gleason KM, McDaniel MR, Feinglass J, et al. Results of the Medications at Transitions and Clinical Handoffs (MATCH) study: an analysis of medication reconciliation errors and risk factors at hospital admission. J Gen Intern Med 2010;25:441-7.

14. Forster AJ, Clark HD, Menard A, et al. Adverse events among medical patients after discharge from hospital. CMAJ 2004;170:345-9.

15. Osterberg L, Blaschke T. Adherence to medication. N Engl J Med. 2005; 353: 487-497.

16. Ho PM, Bryson CL, Rumsfeld JS. Medication adherence: its importance in cardiovascular outcomes. Circulation. 2009;119:3028-35. 
17. Yancy CW, Jannuzzi JL, Allen LA et al. 2017 ACC Expert Consensus Decision Pathway for Optimization of Heart Failure Treatment: Answers to 10 Pivotal Issues About Heart Failure With Reduced Ejection Fraction: A Report of the American College of Cardiology Task Force on Expert Consensus Decision Pathways. J Am Coll Cardiol. 2018;71:201-230.

18. Wu JR, Lennie TA, Dekker RL, Biddle MJ, Moser DK. Medication adherence, depressive symptoms, and cardiac event-free survival in patients with heart failure. J Card Fail. 2013;19:317-24.

19. Junger J, Schellberg D, Muller-Tasch T et al. Depression increasingly predicts mortality in the course of congestive heart failure. Eur J Heart Fail 2005;7:261e7.

20. Nieuwenhuis MM, Jaarsma T, van Veldhuisen DJ, van der Wal MH. Self-reported versus 'true' adherence in heart failure patients: a study using the Medication Event Monitoring System. Neth Heart J. 2012;20:313-9.

21. Mennuni M, Gulizia MM, Alunni G, et al. Position paper ANMCO: Gestione della dimissione ospedaliera [ANMCO Position paper: Hospital discharge planning]. G Ital Cardiol (Rome). 2016;17:657-686.

22. Fitzgerald AA, Powers JD, Ho PM et al. Impact of medication nonadherence on hospitalizations and mortality in heart failure. J Card Fail. 2011;17:664-9.

23. OMS, Therapeutic Patient Education 1998.

24. Fattirolli F, Angelino E. L'informazione essenziale e irrinunciabile alla dimissione dal reparto per acuti dopo sindrome coronarica - Parte 2: Il progetto per migliorare la qualità delle cure. Monaldi Arch Chest Dis 2012;78:138-47.

25. Rogers AM, Ramanath VS, Grzybowski M, et al. The association between guideline-based treatment instructions at the point of discharge and lower 1-year mortality in Medicare patients after acute myocardial infarction. Am Heart J 2007;154:461-9.

26. Crissinger ME, Marchionda KM, Dunlap ME. Adherence to clinical guidelines in heart failure (HF) outpatients: Impact of an interprofessional HF team on evidence-based medication use. J Interprof Care. 2015;29:483-7.

27. Wood DA, Kotseva K, Connolly S et al; EUROACTION Study Group. Nurse-coordinated multidisciplinary, family-based cardiovascular disease prevention programme (EUROACTION) for patients with coronary heart disease and asymptomatic individuals at high risk of cardiovascular disease: a paired, cluster-randomised controlled trial. Lancet. 2008;371:1999-2012.

28. Radini D, Sola G, Zeriali N, et al. Obiettivi, organizzazione e attività di un Ambulatorio Infermieristico Cardiologico di Continuità Assistenziale. G Ital Cardiol 2016;17:377-87.

29. Radini D, Mennuni M, Accardo S, et al. Addenda online Position paper ANMCO: Bisogni assistenziali del malato cardiopatico complesso: dalla fase acuta alla fase cronica. G Ital Cardiol (Rome). 2019;20(10):0. doi:10.1714/3228.32063.

30. Di Lenarda A, Metra M. La rete per il cardiopatico cronico. G Ital Cardiol 2009;10(Suppl 3-6):22S-30S.

31. Schou M, Gislason G, Videbaek L, et al. Effect of extended follow-up in a specialized heart failure clinic on adherence to guideline recommended therapy: NorthStar Adherence Study. Eur J Heart Fail 2014;16:1249-55.

32. Jerant AF, Azari R, Nesbitt TS. Reducing the cost of frequent hospital admissions for congestive heart failure: a randomized trial of a home telecare intervention. Med Care. 2001;39:1234-45.

33. Kornowski R, Zeeli D, Averbuch M et al. Intensive home-care surveillance prevents hospitalization and improves morbidity rates among elderly patients with severe congestive heart failure. Am Heart J. 1995;129: 762-6.

34. Ferrante D, Varini S, Macchia A, et al. Long-term results after a telephone intervention in chronic heart failure: dial (randomized trial of phone intervention in chronic heart failure) follow-up. J Am Coll Cardiol. 2010;56: 372-8.

35. Andrès E, Talha S, Zulfiqar A-A, et al. Current research and new perspectives of telemedicine in chronic heart failure: narrative review and points of interest for the clinician. J Clin Med 2018;7:544.

36. Tse G, Chan C, Gong M, et al.: Telemonitoring and hemodynamic monitoring to reduce hospitalization rates in heart failure: a systematic review and meta-analysis of randomized controlled trials and real-world studies. J Geriatr Cardiol 2018, 15:298-309.

37. Ding EY, Ensom E, Hafer N et al. Point-of-care technologies in heart, lung, blood and sleep disorders from the Center for Advancing Point-of-Care Technologies. Curr Opin Biomed Eng. 2019;11:58-67.

38. Oosterom-Calo R, van Ballegooijen AJ, Terwee CB et al. Determinants of adherence to heart failure medication: a systematic literature review. Heart Fail Rev. 2013;18:409-27.

39. Angelino E, Fattirolli F. L'informazione essenziale e irrinunciabile alla dimissione dal reparto per acuti dopo sindrome coronarica. Parte 1. Monaldi Arch Chest Dis 2012;78:79-84.

40. O'Connor AM, Stacey D, Entwistle V, et al. Decision aids for people facing health treatment or screening decisions. Cochrane Database Syst Rev 2003;CD001431.

41. Koelling TM, Johnson ML, Cody RJ, Aaronson KD. Discharge education improves clinical outcomes in patients with chronic heart failure. Circulation. 2005;111:179-185.

42. Phillips CO, Wright SM, Kern DE et al. Comprehensive discharge planning with postdischarge support for older patients with con gestive heart failure: a meta-analysis. JAMA. 2004;291:1358-1367.

43. Vinson JM, Rich MW, Sperry JC, et al. Early readmission of elderly patients with congestive heart failure. J Am Geriatr Soc 1990;38:1290-5.

44. Hernandez AF, Greiner MA, Fonarow GC et al Relationship between early physician follow-up and 30-day readmission among Medicare beneficiaries hospitalized for heart failure. JAMA. 2010;303:171.

45. Desai AS, Stevenson LW. Rehospitalization for heart failure: predict or prevent?. Circulation. 2012;126:501-506.

46. Lee JL, Maciejewski M, Raju S, Shrank WH, Choudhry NK. Value-based insurance design: quality improvement but no cost savings. Health Aff (Millwood). 2013;32:1251-7. 


\title{
Il modello del disease management nella gestione dello scompenso cardiaco
}

\author{
Ombretta Para \\ Medicina Interna 1, AOU Careggi, Firenze, Italia
}

Lo scompenso cardiaco rappresenta una delle principali cause di morte e invalidità nei paesi occidentali. Inoltre, lo scompenso cardiaco è una patologia cronica che comporta un notevole sforzo da parte delle strutture sanitarie per il costo degli interventi sanitari e la frequenza di ospedalizzazioni, configurandosi come una delle più rilevanti voci della spesa sanitaria $(5 \%$ dei costi totali sostenuti per l'ospedalizzazione e 1-2\% della spesa sanitaria totale). Il progressivo invecchiamento della popolazione e la ridotta mortalità nelle fasi acute della cardiopatia ischemica stanno portando in breve tempo ad un'ulteriore diffusione dello scompenso cardiaco. Sappiamo infatti che la prevalenza di scompenso è correlata all'età e che l'incidenza si raddoppia per ogni decade a partire dai 45 anni e che l'età media di diagnosi di scompenso è di 74 anni. I dati epidemiologici riportano in Europa una prevalenza dell'insufficienza cardiaca (IC) dello 0,4\%-2\%, che aumenta con l'età. Il 50\% dei malati con IC muore entro 5 anni dalla formulazione della diagnosi, il 50\% dei soggetti con fase avanzata di malattia (classe NYHA III-IV) entro 1 anno. L'età avanzata dei malati (media di 76 anni) e l'alta incidenza di comorbilità sono tra le ragioni della prognosi infausta.

Oltre il 44\% dei pazienti ospedalizzati viene nuovamente ricoverato entro sei mesi e di questi quasi il $30 \%$ più di una volta e circa la metà delle ospedalizzazioni interessa soggetti a basso rischio e sarebbe pertanto potenzialmente evitabile. ${ }^{1}$

È necessario pertanto individuare nuovi modelli assistenziali, con l'obiettivo di migliorare la cura di questi pazienti senza eccedere nella spesa sanitaria. Il

Corrispondente: Ombretta Para, Medicina Interna 1, AOU Careggi, Largo Brambilla 3, 50134 Firenze, Italia.

E-mail: ombretta.para@gmail.com

Articolo pubblicato secondo la Creative Commons Attribution NonCommercial 4.0 License (CC BY-NC 4.0).

${ }^{\circ}$ Copyright: the Author(s), 2021

Licensee PAGEPress, Italy

QUADERNI - Italian Journal of Medicine 2021; 9(2):e9 modello assistenziale denominato disease management prevede un approccio multidisciplinare alla cura delle patologie croniche e l'erogazione, durante il decorso naturale della malattia, di interventi terapeutici integrati. $^{2-5}$

I più comuni fattori legati alla reospedalizzazione sono la mancanza aderenza terapeutica domiciliare, il fallimento nel trattare precocemente un peggioramento della sintomatologia, l'inadeguatezza nell'attenersi alle prescrizioni mediche riguardo lo stile di vita ed un inadeguato percorso successivo alla dimissione per la presa in carico del paziente. ${ }^{5}$ I programmi di $d i$ sease management sono nati proprio nell'ottica di migliorare la gestione e l'outcome clinico dei pazienti con scompenso cardiaco.

Nonostante i principali progressi nella farmacoterapia dell'insufficienza cardiaca, i tassi di ospedalizzazione rimangono alti, in gran parte a causa di una moltitudine di fattori psicosociali, comportamentali e finanziari che fungono da barriere all'effettiva osservanza del trattamento prescritto. Per affrontare questi problemi, molti centri hanno adottato un approccio multidisciplinare alla gestione delle malattie dello scompenso cardiaco:6-10 1 'approccio di un team multidisciplinare e l'utilizzo di tecnologie di monitoraggio domiciliare erano in grado di ridurre l'ospedalizzazione, senza incidere sui costi.

I dati emersi dalla revisione sistematica di Rich et al. ${ }^{11}$ condotta su 16 studi riguardanti programmi multidisciplinari di gestione dello scompenso cardiaco (10 erano studi osservazionali non randomizzati e 6 erano studi clinici randomizzati) hanno riportato benefici significativi in termini di riduzione dell'ospedalizzazione. Diversi studi hanno inoltre riportato un miglioramento della qualità della vita, della capacità funzionale, della soddisfazione del paziente e del rispetto della dieta e dei farmaci. In tutti gli studi in cui è stata eseguita un'analisi dei costi è emerso come $\mathrm{i}$ programmi strutturati di gestione dello scompenso cardiaco fossero anche convenienti.

Le grandi limitazione emersa da questa revisione erano legata principalmente alla difficoltà di estendere i risultati pubblicati alle diverse realtà cliniche a causa della vasta ed eterogenea popolazione di pazienti con 
insufficienza cardiaca nella comunità, alla fattibilità di realizzare nella pratica quotidiana specifici programmi di gestione della malattia in diversi ambienti ed all'incertezza legata all'implementazione di una strategia gestionale conveniente anche dal punto di vista economico.

In uno studio successivo, pubblicato su CHEST nel $2002^{12}$ gli autori hanno valutato se la gestione dei pazienti con scompenso cardiaco in un contesto clinico adeguata per breve termine, seguito da un monitoraggio stretto dopo la dimissione al domicilio, fosse associato ad un outcome migliore. Nello studio sono stati arruolati i pazienti dimessi dall'ospedale con una diagnosi primaria di scompenso cardiaco, con disfunzione sistolica ventricolare sinistra e che avessero possibilità di svolgere il percorso di follow-up ambulatoriale anche all'interno del sistema ospedaliero.

I pazienti sono stati poi suddivisi in due gruppi in base al tipo di follow-up intrapreso dopo la dimissione (ambulatorio ospedaliero o ambulatorio del medico di medicina generale).

Nel gruppo in follow-up intra-ospedaliero vi era mediamente un tempo di attesa più breve per la prima visita ambulatoriale dopo la dimissione (11 giorni $v s$ 15 giorni, $\mathrm{P}=0,09)$ e più visite ambulatoriali entro 90 giorni (10 visite vs 2 visite, $\mathrm{P}<0,001$ ). In questo gruppo inoltre il tasso combinato di riammissione ospedaliera e mortalità a 90 giorni $(10 \%$ vs $30 \%$, $\mathrm{P}<0,018)$ e 1 anno $(21 \%$ vs $43 \%, \mathrm{P}<0,02)$ era inferiore, con una riduzione del rischio relativo del $77 \%$ per riammissione ospedaliera a 30 giorni ed un tasso di riammissioni statisticamente inferiore a 90 giorni e 1 anno. Infine, in questi pazienti l'aderenza terapeutica era significativamente più elevata.

Gli autori concludono pertanto affermando che un programma completo e strutturato di follow-up per i pazienti dimessi con una diagnosi di scompenso cardiaco porti ad un minor numero di ospedalizzazioni e ad una migliore sopravvivenza libera da eventi rispetto ai pazienti seguiti dai loro medici di base.

Gli studi condotti negli ultimi 20 anni $^{13-18}$ hanno documentato un netto miglioramento della prognosi dei pazienti affetti da patologie croniche gestiti con questo modello. Le modalità di intervento più spesso messe in atto negli studi ${ }^{19-21}$ erano l'addestramento del paziente, da implementare nel periodo di follow-up mediante frequenti contatti telefonici o visite domiciliari.

La gestione integrata multidisciplinare unita ad un attento monitoraggio domiciliare è in grado di migliorare l'outcome dei pazienti affetti da scompenso cardiaco. La possibilità di avvalersi delle ultime novità tecnologiche nel campo della telemedicina ha reso la gestione a distanza più funzionale sia per il paziente che per i sanitari, con un notevole risparmio economico. Il beneficio economico legato all'utilizzo di tecnologie di ultima generazione deriva infatti dalla riduzione dei costi legati all'ospedalizzazione del paziente con scompenso cardiaco, spesso anziano e con numerose comorbidità, che comporta degenze prolungate e alti costi assistenziali.

Dati di real-life estremamente interessanti sono quelli emersi dallo studio TEMISTOCLE, ${ }^{22}$ studio italiano pubblicato nel 2003, il cui scopo era quello di descrivere profilo clinico, l'uso delle risorse, la gestione e il risultato in una popolazione di pazienti ricoverati nel mondo reale con insufficienza cardiaca.

Attraverso un'indagine trasversale prospettica sui ricoveri ospedalieri per scompenso cardiaco acuto, gli autori hanno valutato le differenze generali nelle caratteristiche del paziente, nel percorso diagnostico, nel trattamento e nell'outcome ospedaliero di 2127 pazienti con scompenso cardiaco ammessi in 167 dipartimenti di cardiologia e 250 reparti di medicina interna. Dai dati dello studio TEMISTOCLE è emerso come pazienti ammessi alle unità di cardiologia fossero mediamente più giovani $(56,3 \%>70$ anni vs $76,2 \%, \mathrm{P}<0,0001)$, con sintomi più gravi (NYHA IV $35 \%$ vs $29 \%, \mathrm{P}=0,00014$ ), e venivano più spesso sottoposti a valutazione della funzione ventricolare $(89,3 \%$ vs $54,8 \%, \mathrm{P}<0,0001)$ e angiografia coronarica $(7,5 \%$ vs $0,9 \%, \mathrm{P}<0,0001)$ rispetto a quelli ricoverati in unità di medicina interna. Nei reparti di cardiologia inoltre, erano più spesso prescritti $\beta$-bloccanti $(17,8 \%$ vs $8,7 \%, \mathrm{P}<0,0001)$. Tuttavia, la prescrizione di inibitori dell'enzima di conversione dell'angiotensina e bloccanti del recettore dell'angiotensina $(78,7 \%$ vs $81,5 \%, \mathrm{P}=$ non significativo [NS]) e la mortalità ospedaliera $(5,2 \%$ vs $5,9 \%, \mathrm{P}=\mathrm{NS})$ erano simili. Una visita di follow-up di 6 mesi è stata eseguita nel $56,4 \%$ dei casi $(68,2 \%$ dei pazienti cardiologici $v s$ $49,4 \%$ dei pazienti in medicina, $\mathrm{P}<0,0001)$. I tassi di riammissione a 6 mesi $(43,7 \%$ vs $45,4 \%, \mathrm{P}=\mathrm{NS})$ e mortalità $(13,9 \%$ vs $16,7 \%, \mathrm{P}=\mathrm{NS})$ erano invece simili nei due diversi setting assistenziali.

I dati del TEMISTOCLE inoltre dimostravano come quasi la metà dei pazienti ricoverati per scompenso cardiaco acuto, entro l'anno vada incontro ad un nuovo ricovero ospedaliero, e che nel 50\% dei casi, il ricovero era dovuto ad inosservanza o incomprensione delle istruzioni mediche ed alla scarsa aderenza terapeutica. Nell' $80 \%$ dei soggetti instabili il nuovo ricovero si verifica entro 3 mesi dalla dimissione.

È chiaro quindi che se il paziente viene dimesso dall'ospedale senza un chiaro percorso di sorveglianza continua potrà non seguire in maniera corretta le indicazioni ricevute riguardo il cambiamento dello stile di vita ed a non assumere in maniera regolare la terapia domiciliare, soprattutto se caratterizzata da numerosi farmaci. La mancata aderenza ad uno stile di vita adeguato ed alla terapia impostata in ambiente ospedaliero porterà inevitabilmente a frequenti riacutizzazioni cliniche. Inoltre un adeguato percorso di sorveglianza dopo la dimissione dovrebbe garantire la titolazione di 
alcuni farmaci, che non può avvenire durante la fase acuta, o le modificazioni terapeutiche necessarie in un quadro cronico che spesso si configura nell'ambito di numerose comorbidità.

È quindi ipotizzabile che attraverso un percorso strutturato di disease management, mettendo in atto interventi educativi che possano aiutare ad ottimizzare l'aderenza alla terapia, si potrebbero prevenire molte riacutizzazioni con conseguente necessità di ospedalizzazione.

Abbiamo visto come nella gestione del paziente con scompenso cardiaco siano state sviluppati diversi modelli di cura. Sicuramente possiamo identificare due grandi setting assistenziali: il primo che vede il ruolo dell'ospedale e del suo personale come centrale nel follow-up dei pazienti (modello ospedalocentrico) ed il secondo che invece affida la responsabilità a figure professionali extraospedaliere (medico di medicina generale) ed è regolato da un'organizzazione basata sul territorio (modello territoriale). Entrambi i sistemi devono necessariamente seguire specifici protocolli.

Dalla letteratura si evince che i programmi ospedalocentrici siano organizzati in maniera diversa, talvolta prevedendo il ruolo centrale dello specialista cardiologo ospedaliero, altre volte prevedendo la collaborazione tra ospedale e territorio. Purtroppo non tutti i reparti ospedalieri hanno risorse tali da garantire un adeguato follow-up ad un elevato numero di pazienti. In genere, negli studi i pazienti che venivano affidati al percorso di follow-up ospedaliero erano pazienti con patologia cardiaca più grave, con marcata compromissione della funzione sistolica ventricolare sinistra e molteplici comorbidità. Inoltre un punto debole del modello ospedalocentrico è il ruolo marginale che potrebbe assumere il medico di medicina generale che invece dovrebbe essere cruciale nella gestione domiciliare del paziente fragile pluripatologico.

Il gruppo di ricerca geriatrica di Brescia ${ }^{23}$ ha proposto un modello di cura definito leggero basato sul ruolo centrale dell'ospedale è stato adottato dal nostro gruppo. In questa esperienza sono stati arruolati 579 pazienti con diagnosi di scompenso cardiaco $(25,7 \%$ dei quali in classe NYHA III e IV), consecutivamente dimessi dai 24 letti di unità geriatrica per acuti. La mortalità a sei mesi è stata del 33,6\% (50 pazienti in classe NYHA III e IV). Il follow-up era basato su un'accurata informazione del medico curante (lettera dettagliata alla dimissione), su un controllo clinico ambulatoriale mensile e sull'educazione del paziente e dei familiari per una rigida compliance ai farmaci, al regime dietetico ed al controllo del peso corporeo. Il programma, sebbene abbia prodotto risultati meno favorevoli rispetto a quelli segnalati in letteratura, potrebbe secondo gli autori rappresentare un buon compromesso per il trattamento dei pazienti con scompenso cardiaco.

Negli Stati Uniti molte organizzazioni sanitarie, con lo scopo di contenere i costi, hanno promosso strategie atte a valorizzare il ruolo dei medici di famiglia, limitando il ricordo alle cure specialistiche.

Gli interventi messi in atto consistevano in un'educazione globale del paziente e del caregiver, nella prescrizione di una dieta idonea, nell'eventuale attivazione dei servizi sociali, nella pianificazione di una dimissione precoce ed in un follow-up ravvicinato. Nel gruppo così gestito si è rivelato minore il numero di riospedalizzazioni per scompenso cardiaco, con dati positivi rispetto alla qualità di vita ed alla riduzione dei costi.

L'importanza di un modello di gestione integrata trova riscontro anche nell'evidenza che i pazienti con scompenso cardiaco necessitano di supporto emotivo e che questo è un forte predittore indipendente di eventi cardiovascolari fatali e non fatali. Quindi, è necessario un continuo supporto a lungo termine dopo la dimissione dall'ospedale con un programma di cura prenda in considerazione questi aspetti soprattutto per i pazienti che non hanno un adeguato supporto familiare.

Il telemanagement, uno specifico tipo di telemedicina che utilizza la telecomunicazione per realizzare interventi di trattamento in assenza di contatto personale, anche in epoca pandemica come quella che stiamo vivendo, potrebbe permettere il superamento della separazione tra ospedale e territorio e potrebbe funzionare come aiuto in situazioni particolarmente critiche, in cui vi sia la necessità di supportare il personale del territorio ed i caregiver familiari.

Per quanto riguarda l'esperienza italiana dati interessanti emergono dal Boario Home Care Project, ${ }^{24-26}$ che integra tre componenti (contatto telematico con il medico di medicina generale, il telenursing e la telediagnosi, con trasmissione dell'ecocardiogramma e sua refertazione a distanza) utilizzate come supporto al monitoraggio di pazienti affetti da patologie croniche residenti in aree disagiate e non raggiunte dai servizi ambulatoriali tradizionali

Un altro interessante progetto italiano è il progetto ICAROS (Integrated Care vs Conventional intervention in Cardiac Failure Patients: Randomized Open Label Study - finanziamento FIRB n. RBNE01KYE4 2003) che prevede la gestione domiciliare di pazienti instabili.

ICAROS è un progetto di telemonitoraggio domiciliare cardiologico e psicologico in pazienti con scompenso cardiaco moderato/severo che si basa da un lato su consolidate tecnologie informatiche e di telecomunicazione, e dall'altro su emergenti tecnologie, che consentono la registrazione di dati dal paziente senza restrizioni della sua mobilità e con approccio senza fili (wireless).

L'obiettivo del progetto ICAROS è stato proprio quello di verificare se un intervento integrato sia in grado di ottimizzare/individualizzare la terapia, mi- 
gliorare la compliance terapeutica del paziente, andando ad agire così sia sullo stato funzionale del paziente che sulla sua qualità di vita, ridurre le ospedalizzazioni e la mortalità. ${ }^{27}$

Il modello proposto dallo studio ICAROS (che comporta maggiore complessità di gestione e costi superiori) costituirebbe la fase di telemonitoraggio intensivo, temporalmente limitato, per i periodi di instabilizzazione o per i pazienti più gravi, in fase avanzata. Il ruolo dello psicologo, oltre a rassicurare il paziente e i familiari, è in grado insieme ad un'assistenza integrata di motivare il paziente ad intraprendere un percorso che, nonostante l'iniziale sforzo di apprendimento, è percepito come fondamentale nella gestione del proprio stato di salute per migliorare la propria qualità di vita e quella di tutto il contesto familiare. ${ }^{27}$

La telemedicina applicata ai pazienti con scompenso cardiaco si è rivelata uno strumento utile per la registrazione e la trasmissione, l'archiviazione e l'interpretazione a distanza di parametri cardiovascolari e/o immagini diagnostiche utili per consentire il monitoraggio domiciliare intensivo di pazienti con insufficienza cardiaca avanzata o durante la fase vulnerabile post-acuta, migliorare la prognosi e la qualità della vita del paziente. ${ }^{28}$

L'adozione di modelli più efficienti per la cura dei pazienti, delineata dal disease management, si propone come soluzione al continuo taglio dei costi sanitari, imposti dal progressivo invecchiamento della popolazione. ${ }^{27}$

In conclusione sono sicuramente necessari nuovi modelli assistenziali, integrati e che si avvalgano di nuove tecnologie per migliorare il livello di cura senza gravare sui costi del sistema sanitario.

Nella gestione del paziente con scompenso cardiaco il modello del disease management, attraverso un approccio multidisciplinare e l'erogazione di interventi mirati ed integrati, si è rivelato efficace sia in termini di miglioramento della qualità di vita, sia di riduzione dei costi sanitari, anche nei pazienti anziani e fragili.

Tuttavia i dati della letteratura sui modelli integrati per la cura dei pazienti con scompenso cardiaco non possono ancora fornire indicazioni conclusive e sarà necessario implementare in futuro modelli gestionali integrati e telematici da validare nell'ambito di studi clinici ben strutturati, per identificare la migliore modalità di cura globale in grado di modificare realmente la qualità e l'aspettativa di vita specialmente dei pazienti più fragili e complessi.

\section{Bibliografia}

1. Mangia R, Senni M, Cacciatore G, et al. È tempo di organizzare una "nuova" lotta allo scompenso cardiaco? Italian Heart Journal Suppl 2003; 4: 232-236.
2. Rich MW. Heart failure disease management: a critical review. J Card Fail 1999; 5: 64-75.

3. Cline CM, Israelsson BY, RB, Broms K, Erhardt LR. Cost effective management programme for heart failure reduces hospitalisation. Heart 1998; 80: 442-6.

4. Schulman KA, Mark DB, Califf RM. Outcomes and costs within a disease management program for advanced congestive heart failure. Am Heart J 1998; 135: 285-92.

5. Whellan DJ, Gaulden L, Gattis WA, et al. The benefit of implementing a heart failure disease management program. Arch Intern Med 2001; 161: 2223-8

6. Andrea Takeda, Nicole Martin, Rod S Taylor, Stephanie JC Taylor, Cochrane Heart Group Disease management interventions for heart failure Cochrane Database Syst Rev. 2019 Jan; 2019(1): CD002752. Published online 2019 Jan 8. doi: 10.1002/14651858.CD002752.pub4

7. de Lusignan S, Wells S, Johnson P, Meredith K, Leatham E. Compliance and effectiveness of 1 year's home telemonitoring. The report of a pilot study of patients with chronic heart failure. Eur J Heart Fail 2001; 3: 723-30. 20.

8. Krumholz HM, Parent EM, Tu N, et al. Readmission after hospitalization for congestive heart failure among Medicare beneficiaries. Arch Intern Med 1997; 157: 99104. 21.

9. Cleland JG, Cohen-Solal A, Aguilar JC, et al, for the IMPROVEMENT of Heart Failure Programme Committees and Investigators. Improvement programme in evaluation and management; Study Group on Diagnosis of the Working Group on Heart Failure of the European Society of Cardiology. Management of heart failure in primary care (the IMPROVEMENT of Heart Failure Program): an international survey. Lancet 2002; 360: 1631-9. 22.

10. Kornowsky R, Zeeli D, Averbuch M, et al. Intensive homecare surveillance prevents hospitalization and improves morbidity rates among elderly patients with severe congestive heart failure. Am Heart J 1995; 129: 762-6.

11. Rich MW. Heart failure disease management: a critical review. J Card Fail 1999; 5: 64-75

12. Akosah KO, Shaper AM, Havlik P, Barnhart S, Devine $\mathrm{S}$. Improving care for patients with chronic heart failure in the community. The importance of a disease management program. Chest 2002; 122: 906-12.

13. Stewart S, Marley JE, Horowitz JD. Detecting early clinical deterioration in chronic heart failure patients postacute hospitalisation: a critical component of multisciplinary, home-based intervention? Eur J Heart Fail 2002; 4: 345-51. 12.

14. Grady KL, Dracupp K, Kennedy G, Moser DK, et al. Team management of patients with heart failure: a statement for healthcare professionals from the Cardiovascular Council of the American Heart Association. Circulation 2000; 102: 2443-56. 13.

15. Shah NB, Der E, Ruggiero C, Heidenreich PA, Massie BM. Prevention of hospitalizations for heart failure with an interactive home monitoring program. Am Heart J 1998; 135: 373-8. 14.

16. Stewart S, Marley JE, Horowitz JD. Effects of a multidisciplinary, home-based intervention on unplanned readmissions and survival among patients with chronic congestive heart failure: a randomised controlled study. Lancet 1999; 354: 1077-83.

17. Stewart S, Horowitz JD. Home-based intervention in con- 
gestive heart failure. Long-term implications on readmission and survival? Circulation 2002; 105: 2861-6.

18. GESICA Investigators. Randomised trial of telephone intervention in chronic heart failure: the DIAL trial. BMJ 2005; 331: 425.

19. Ekman I, Andersson B, Ehnfors M, et al. Feasibility of a nurse-monitored, outpatientcare programme for elderly patients with moderate-to-severe, chronic heart failure. Eur Heart J 1998; 8: 1254-60.

20. Naylor MD, Brooten D, Campbell R, et al. Comprehensive discharge planning and home follow-up of hospitalised elders. A randomised clinical trial. JAMA 1999; 281: 613-20.

21. Rich MW, Beckham V, Wittenberg C, Leven CL, Freedland KE, Carney RM. A multidisciplinary intervention to prevent the readmission of elderly patients with congestive heart failure. N Engl J Med 1995; 333: 1190-5

22. Di Lenarda A, Scherillo M, Maggioni AP, et al, for the TEMISTOCLE Investigators. Current presentation and management of heart failure in cardiology and internal medicine hospitals units: a tale of two worlds - the TEMISTOCLE study. Am Heart J 2003; 146: E12.

23. Rozzini R, Sabatini T, Costa T, Trabucchi M, Il trattamento dello scompenso cardiaco nell'anziano: modelli di cura a confronto Fondazione Poliambulanza, Brescia. Geriatria \& Pratica Medica 2004; (2):8-10

24. Scalvini S, Volterrani M, Giordano A, Glisenti F. Boario Home Care Project: an Italian telemedicine experience. Monaldi Arch Chest Dis 2003; 60: 254-7.

25. Scalvini S, Zanelli E, Volterrani M, et al. A pilot study of nurse-led, home-based telecardiology for patients with chronic heart failure. J Telemed Telecare, 2004; 10: 113-7.

26. Scalvini S, Capomolla S, Zanelli E, et al. Effect of homebased telecardiology on chronic heart failure: costs and outcomes. J Telemed Telecare 2005; 11 (Suppl 1): 16-8.

27. Villani A, Malfatto G, Della Rosa F, et al. Continuità assistenziale nei pazienti con scompenso cardiaco: ruolo delle tecnologie wireless per la telemedicina. Il progetto ICAROS. G Ital Cardiol Vol 8 Febbraio 2007.

28. Di Lenarda A, Casolo G, Gulizia MM, et al. The future of telemedicine for the management of heart failure patients: a Consensus Document of the Italian Association of Hospital Cardiologists (A.N.M.C.O), the Italian Society of Cardiology (S.I.C.) and the Italian Society for Telemedicine and eHealth (Digital S.I.T.) Eur Heart J Suppl. 2017 May; 19(Suppl D): D113-D129. 


\title{
Modelli a confronto nei percorsi gestionali dello scompenso cardiaco: l'esperienza dell'AO Ordine Mauriziano di Torino
}

\author{
Claudio Norbiato, Luisa Arnaldi, Stefania Marengo, Marinella Tricarico, Manuela Daddea, Cristina Garza, \\ Emanuela Irene, Pietro Paolì
}

SC Medicina Interna, AO Ordine Mauriziano Medicina Interna, Torino (TO), Italia

\section{Introduzione}

L'ambulatorio dello scompenso cardiaco della Medicina Interna dell'AO Ordine Mauriziano nasce nel 2004, fortemente voluto dall'allora Direttore della SC, il Dr. Rodolfo Cavaliere, a seguito dell'evidenza che l'analisi dei ricoveri ospedalieri nazionali mostrava che il DRG 127 (insufficienza cardiaca e shock) era divenuto, nel 2003, la prima causa di ricovero dopo il parto. La percentuale di pazienti riospedalizzati entro 3-6 mesi dalla dimissione risultava molto elevata e oscillava dal 10 al $50 \%$ a seconda delle casistiche; questo fenomeno rendeva lo scompenso cardiaco una delle più rilevanti voci della spesa sanitaria nazionale (5\% dei costi totali sostenuti per l'ospedalizzazione e $1-2 \%$ della spesa sanitaria totale). Nell'anno $2004 \mathrm{si}$ erano registrate in Piemonte 11.639 dimissioni in regime ordinario per DRG 127 (fonte Ministero della Salute), che rappresentavano il 2,2\% del totale dei ricoveri; il $63 \%$ di questi ricoveri avveniva nelle Medicine Interne. ${ }^{1}$

Nei primi anni di attività all'ambulatorio dello scompenso cardiaco afferivano esclusivamente pazienti dimessi dal nostro reparto, che venivano presi in carico dai 2 medici internisti che gestivano l'ambulatorio con un follow-up di visite periodiche. Negli anni il modello organizzativo si è evoluto con una progressiva implementazione dei servizi coinvolti, un ampliamento dell'organico medico e della dotazione strumentale ed un coinvolgimento strutturato della componente infermieristica.

Corrispondente: Claudio Norbiato, SC Medicina Interna, AO Ordine Mauriziano, via Magellano 1, 10128 Torino, Italia. E-mail: cnorbiato@mauriziano.it

Articolo pubblicato secondo la Creative Commons Attribution NonCommercial 4.0 License (CC BY-NC 4.0).

${ }^{\circ}$ Copyright: the Author(s), 2021

Licensee PAGEPress, Italy

QUADERNI - Italian Journal of Medicine 2021; 9(2):e10

\section{Percorso del paziente}

L'attuale setting prevede un accesso più articolato all'ambulatorio: in aggiunta ai pazienti dimessi dal nostro reparto afferiscono al servizio anche pazienti inviati dal DEA della nostra Azienda Ospedaliera e pazienti inviati dai Medici di Medicina Generale che operano sul territorio di nostra competenza.

Dal punto di vista organizzativo abbiamo identificato come collettore per le diverse provenienze dei pazienti il Day Service di Medicina Interna, che ben si presta a svolgere questa attività di assistenza ambulatoriale complessa e integrata, finalizzata ad erogare in regime ambulatoriale e in tempi brevi, pacchetti di prestazioni multidisciplinari con finalità diagnostico/ clinico/terapeutiche. ${ }^{2}$

La Mission del Day Service di Medicina Interna dell'AO Ordine Mauriziano per il PACC Scompenso Cardiaco è di far eseguire tutti gli accertamenti clinico/strumentali/laboratoristici necessari in tempi brevi (l'obiettivo è in 1 giorno).

\section{Il reparto di Medicina Interna}

Per i pazienti che vengono ricoverati per scompenso cardiaco la cartella clinica informatizzata prevede una griglia di accettazione nella quale devono venir inseriti l'eziologia e la causa precipitante dello scompenso, i parametri ecocardiografici, nonché il valore del peso corporeo ed il valore del BNP all'ingresso. Se il paziente non ha effettuato un ecocardiogramma - o se le condizioni cliniche lo richiedono - l'esame viene eseguito dall'internista durante la degenza.

Il paziente ricoverato per scompenso cardiaco viene segnalato al momento dell'ingresso in reparto, in occasione del briefing mattutino medico-infermieristico, all'equipe infermieristica dedicata (coordinatrice di reparto e 2 CPSI) per la presa in carico.

La lettera di dimissione dal reparto è attualmente una lettera integrata medico-infermieristica. Nella lettera di dimissione medica vengono segnalati, oltre ai valori in uscita dei parametri rilevati all'ingresso (peso 
corporeo e BNP), il tipo di ritmo cardiaco, l'eventuale presenza di turbe della conduzione e/o la presenza di devices (PM, ICD, CRT), la classe NYHA ed i principali indici prognostici (frequenza cardiaca, funzionalità renale, valori di $\mathrm{Hb}$, MAGGIC score). ${ }^{3}$ Nella lettera viene indicata anche la data del primo controllo ambulatoriale, che verrà eseguito in regime di DayService nell'arco di 15 giorni. La lettera di dimissione infermieristica comprende una valutazione dell'autonomia nelle $\mathrm{ADL}$, della capacità di assunzione della terapia e compliance nei riguardi della terapia stessa e un riepilogo dell'attività educazionale svolta durante la degenza ospedaliera. Alla dimissione viene consegnato al paziente un libretto informativo, che il paziente dovrà portare con sé in occasione dei successivi controlli ambulatoriali, con le istruzioni per una corretta ed utile compilazione (Figura 1).

\section{Day Service di Medicina Interna}

Si accede su proposta del Medico Internista, che durante il ricovero ospedaliero segnalerà il paziente all'equipe infermieristica per la presa in carico dal punto di vista educazionale e al momento della dimissione contatterà il Day Service Internistico per la successiva gestione organizzativa.
Nel caso che il paziente venga inviato dal DEA, il Medico Urgentista al momento della dimissione contatterà il Day Service e provvederà a compilare sul sistema informatico aziendale una ricetta dematerializzata con richiesta di visita ambulatoriale.

Il Medico di Medicina Generale a sua volta può richiedere una prestazione di Day Service con Prescrizione SSN di Visita di medicina generale, e quesito clinico valutazione di paziente affetto da scompenso cardiaco.

Nel Day Service Internistico per lo Scompenso Cardiaco vengono erogate le seguenti prestazioni: esami ematochimici, elettrocardiogramma, ecocardiogramma, ecografia internistica (addome, torace, tiroide), ecocolordoppler venoso ed arterioso (grossi vasi addominali, arterie renali, tronchi sovraaortici), terapia educazionale. Tutte queste prestazioni vengono eseguite dal personale medicoinfermieristico che gestisce l'ambulatorio dello scompenso cardiaco.

Il Day Service di Medicina Interna ha attualmente in carico più di 600 pazienti; i pazienti che afferiscono all'ambulatorio dello scompenso cardiaco risultano 122 $(48 \% \mathrm{M} \mathrm{e} 52 \% \mathrm{~F})$, con un'età media di 83 anni.

La prenotazione presso il Day Service Medicina Interna può essere fatta telefonicamente (numero dedicato: 011-5082884) o tramite indirizzo e-mail:

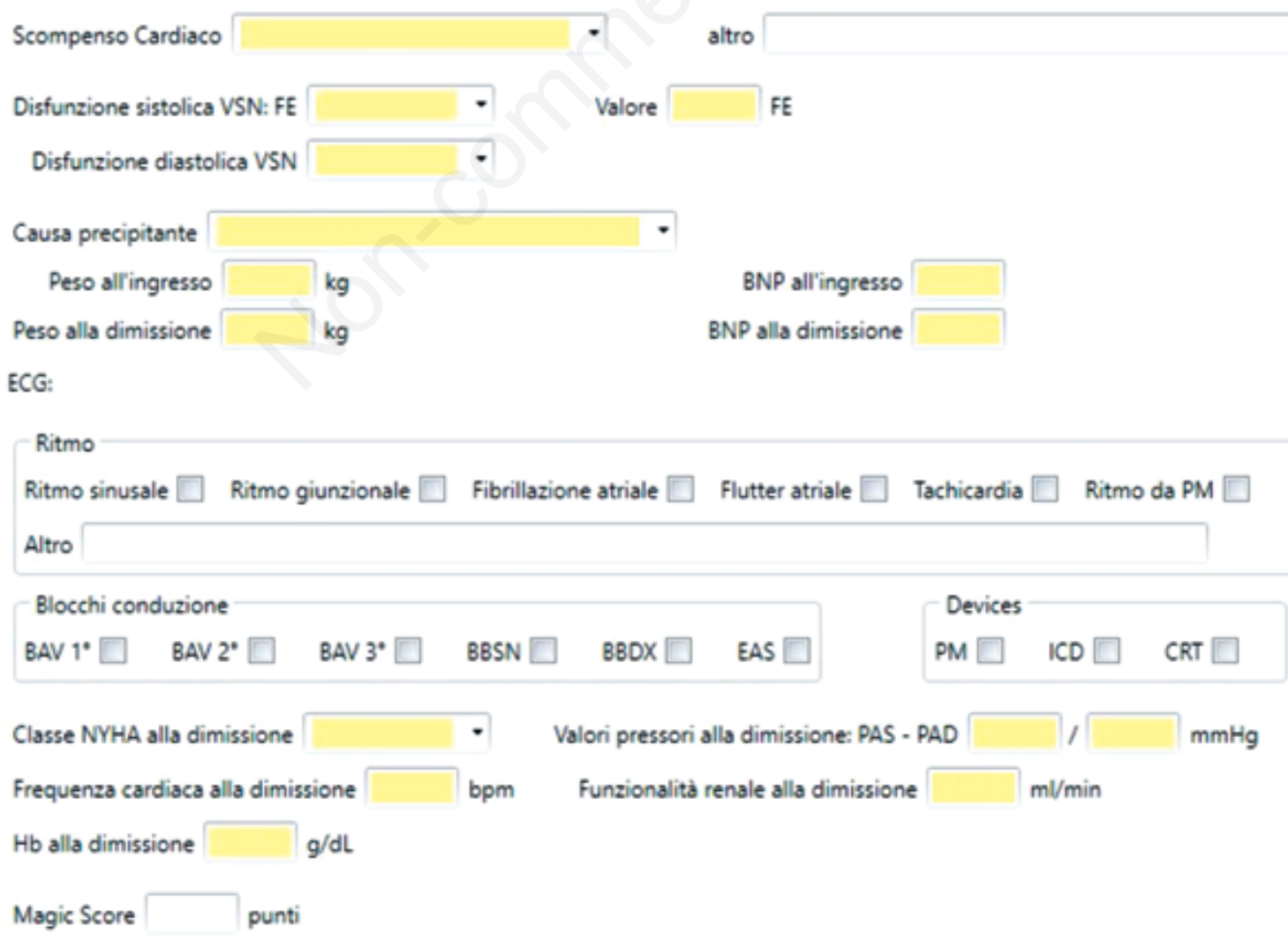

Figura 1. Lettera di dimissione. 
dayservice@mauriziano.it oppure scompensomedicina @mauriziano.it (Figura 2).

\section{Ambulatorio dello scompenso cardiaco}

L'ambulatorio internistico dello Scompenso Cardiaco è funzionante in orario pomeridiano tre giorni a settimana: martedì, mercoledì e giovedì dalle 14:00 alle 16:30

L'equipe medico-infermieristica è composta da 3 medici - tutti con competenze di ecografia internistica certificate; uno di questi con certificazione S.I.E.C. di ecografia cardiovascolare - e 3 infermieri: due facenti parte dell'equipe del reparto di Medicina Interna ed il terzo responsabile del Day Service.

La prima visita - preceduta da un'intervista telefonica effettuata dal personale infermieristico a distanza di una settimana dalla dimissione - viene eseguita, entro 15 giorni, in Regime di Day Service con contestuale esecuzione di esami ematochimici (emocromo, funzionalità epatica e renale, elettroliti, BNP, coagulazione se necessario) ed ecografia toracica. In occasione della prima visita saranno presenti sia il medico che l'infermiere ed il paziente porterà in visione il diario di monitoraggio con le misure antropometriche prese al domicilio (pressione arteriosa, peso e diuresi). È possibile contestualmente eseguire - se le condizioni cliniche lo richiedono - elettrocardiogramma ed ecocardiogramma di controllo. L'ambulatorio prevede un'attività condivisa per la gestione dei pazienti complessi con gli specialisti endocrinologi, pneumologi e nefrologi, i quali vengono coinvolti di volta in volta sui singoli pazienti. Con cadenza mensile viene effettuato un ambulatorio congiunto internistico-cardiologico per la valutazione collegiale dei pazienti che possono avere indicazione a studio emodinamico e/o all'impianto di devices.

In base al quadro clinico e al profilo di rischio il successivo controllo (in regime ambulatoriale, di Day Service o in forma di telemedicina) verrà eseguito a 1 o 3 mesi e i successivi a 3 o 6 mesi. In caso di peggioramento clinico è disponibile il numero di Day Service al quale il paziente può chiamare per una valutazione d'urgenza.

Dal mese di novembre pv inizierà una sperimentazione di telemedicina che vedrà coinvolti nella nostra AO l'ambulatorio dello scompenso cardiaco della Medicina Interna e l'ambulatorio delle malattie renali avanzate della Nefrologia; la piattaforma impiegata consentirà l'effettuazione della televisita (tramite l'impiego di PC o Smartphone da parte del paziente) e la condivisione di esami di laboratorio e/o strumentali. Riteniamo che questa nuova modalità di fornire prestazioni sanitarie a distanza, quanto mai necessaria in questo momento per scongiurare l'affollamento degli Ospedali, si rivelerà molto utile per una importante quota dei nostri pazienti cronici e fragili che presentano difficoltà negli spostamenti e che necessitano di controlli periodici di parametri clinici e di esami laboratoristici.

I pazienti che afferiscono all'ambulatorio dello scompenso cardiaco e che sono in terapia anticoagulante orale con DOACs vengono presi in carico sempre dal nostro Day Service per l'effettuazione dei

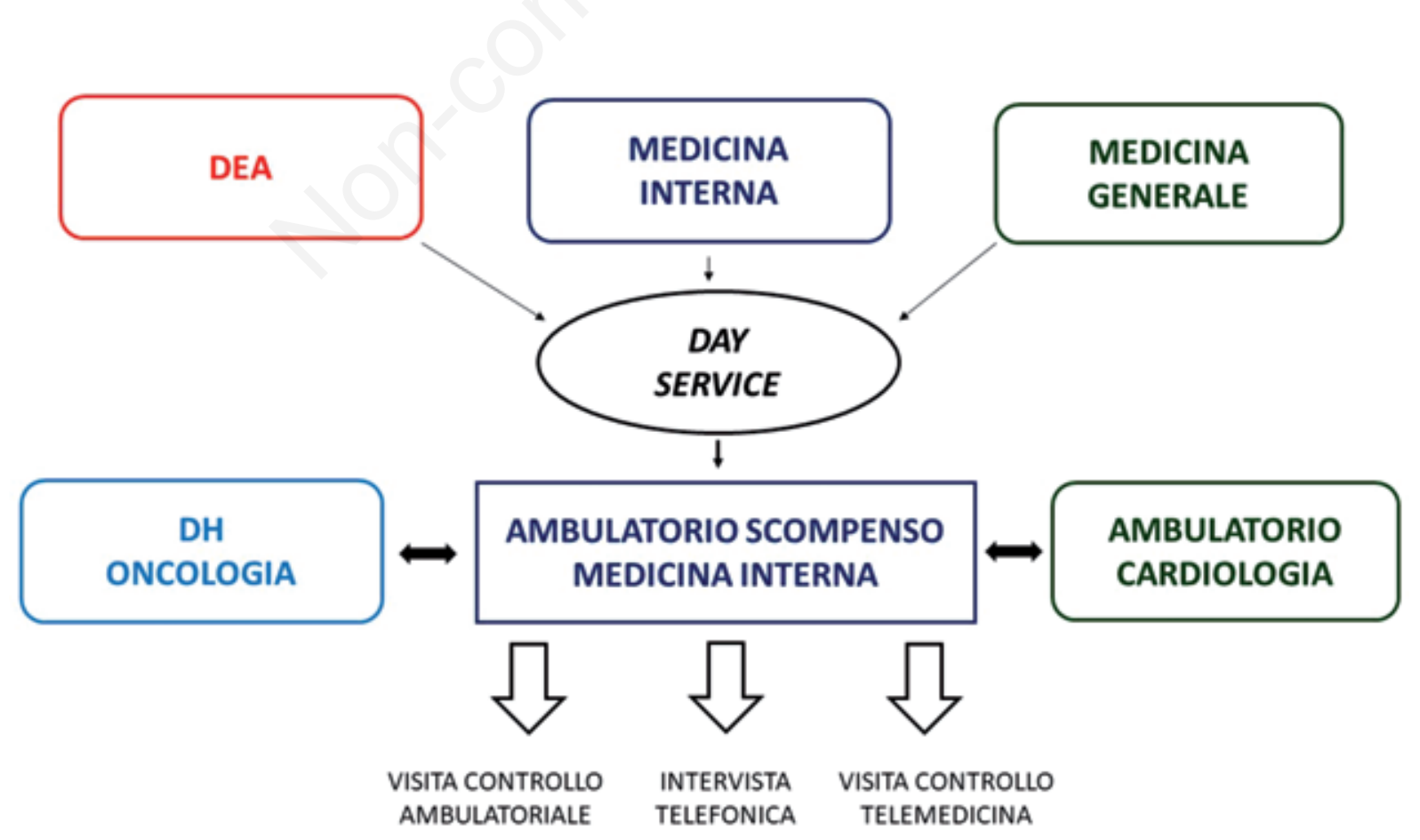

Figura 2. Percorso del paziente. 
controlli laboratoristici programmati ed il rinnovo dei piani terapeutici.

L'ultima attività, che si è recentemente aggiunta è la collaborazione con la SC di Oncologia per la gestione delle problematiche internistiche e cardiologiche dei pazienti oncologici che afferiscono al DH; sia per la stadiazione del rischio cardiovascolare prima dell'avvio della terapia (chemioterapia, radioterapia) che per il monitoraggio dei potenziali effetti cardiotossici farmaco-indotti, sia nella fase precoce che nella fase tardiva della terapia.

\section{Il ruolo dell'infermiere}

La presa in carico infermieristica ambulatoriale dei pazienti affetti da scompenso cardiaco inizia prima della loro dimissione dalle nostre unità di degenza, nell'ottica di costruire un solido rapporto terapeutico e di fiducia con il paziente e i suoi caregivers. Tale rapporto promuove la centralità del malato e della sua qualità di vita, cercando di favorire una gestione domiciliare della patologia cronica, riducendo gli accessi in pronto soccorso e i ricoveri in ospedale.

I degenti con diagnosi di scompenso cardiaco, di nuovo riscontro o con malattia cronica, vengono segnalati all'equipe ambulatoriale dal personale medico infermieristico del reparto di Medicina Interna, in modo da consentire un percorso educativo con il paziente e il caregiver.

Attualmente sono due le infermiere del reparto di medicina interna, con specifica formazione ed esperienza pluriennale in ambito internistico, che collaborano con l'equipe multidisciplinare dell'ambulatorio. Presto verrà implementato il numero di colleghi infermieri che si occuperanno di questa fase pre-dimissione, con il fine di offrire questo servizio ad un numero sempre maggiore di pazienti e di assicurare una maggiore probabilità di successo nella futura gestione ambulatoriale.
La precocità della presa in carico consente di incrementare la compliance terapeutica promuovendo un corretto stile di vita del paziente per sviluppare le capacità di self care e l'autonomia nella gestione di questa patologia. L'intervento è finalizzato ad attivare un processo di empowerment; in questa fase si procede ad una vera e propria educazione terapeutica e sanitaria che tenda ad acquisire o migliorare le conoscenze ed abilità degli assistiti in modo da poter poi sfruttare la rete di risorse che vengono loro messe a disposizione dopo la dimissione.

Gli interventi pianificati prevedono anche la consegna un libretto informativo e un diario in cui registrare i parametri che si consiglia di monitorare (pressione arteriosa, diuresi, peso, edemi o presenza di dispnea).

Dopo una settimana dalla dimissione avviene un contatto telefonico: attraverso un'intervista strutturata in poche e semplici domande (secondo evidenze di letteratura) si valuta il grado di compliance del paziente. In particolare si indaga l'aderenza alla terapia e le capacità di automonitoraggio secondo quanto consigliato alla dimissione (Figura 3).

Entro quindici giorni dal rientro a domicilio si garantisce una prima visita medico - infermieristica durante la quale vengono misurati i valori antropometrici, controllate le rilevazioni domiciliari effettuate dal paziente e rinforzati i concetti educativi forniti in precedenza. In questa occasione vengono compilate le scale per la valutazione multidimensionale del paziente (Self care of heart failure Index, BADL, IADL, Short Portable Mental Status Questionnaire, Scala di Morinsky, Mini Nutritional Assessment; Scala di Conley, Euro$Q o L)^{4}$ che prevedono una rivalutazione a tre, sei e dodici mesi e facilitano la presa in carico infermieristica oggettivando le skills acquisite dai pazienti e facendo emergere le loro difficoltà.

$\mathrm{Al}$ termine viene stabilita insieme al medico la periodicità dei follow up ambulatoriali e dei contatti te-

\begin{tabular}{|l|}
\hline 1. Quanto pesa oggi? \\
\hline 2. E riuscito/a a rilevare la pressione in questa settimana? \\
\hline 3. Quante volte? (chiedere ultimi valori rilevati) \\
\hline 4. Ha notato un aumento del gonfiore in gambe e caviglie? \\
\hline 5. Ha dovuto aggiungere dei cuscini per dormire meglio? \\
\hline 6. Sente una sensazione di affanno quando compie qualche sforzo? \\
\hline 7. Si è mai dimenticato di assumere i farmaci prescritti ? \\
\hline 8. E occosasionalmente poco attento nell'assunzione dei farmaci? \\
\hline 9. Quando si sente meglio, a volte; interrompe la terapia? \\
\hline 10. E quando di sente peggio? \\
\hline 11. Rispetto all'ultimoincotro/telefonata si sente soggettivamente meglio/peggio \\
\hline
\end{tabular}

Figura 3. Intervista telefonica. 
lefonici che si intervallano ad esse. Questa varia in base al grado di stabilità/instabilità clinica e della compliance del paziente rilevati durante le visite e i contatti infermieristici telefonici. L'infermiere autonomamente decide di anticipare o meno l'accesso in ambulatorio valutando le informazioni ricevute dal colloquio telefonico e procede alla prenotazione della visita.

Ogni intervento è refertato una cartella medico infermieristica integrata ed informatizzata. Viene riconosciuta una prestazione infermieristica di educazione sanitaria il cui contenuto è sempre fruibile al team dell'ambulatorio e al personale dell'azienda che per qualsiasi motivo accede alla cartella ambulatoriale online assicurando una presa in carico continua e costante per i nostri assistiti.

\section{Bibliografia}

1. Profilo Integrato di Cura dello Scompenso cardiaco. AReSS Piemonte; 2009.

2. Ministero della Salute. Mattoni SSN-Mattone 3-Evoluzione del Sistema DRG Nazionale; 2007.

3. Mortara A, Gulizia MM, et al. Documento di consenso ANMCO/FADOI/SIAARTI/SIC/SIMG/SIMI/SIMEU: Il percorso clinico-diagnostico e terapeutico del paziente con scompenso cardiaco acuto dal domicilio alla dimissione dal Pronto Soccorso/Dipartimento di Emergenza-Accettazione. G Ital Cardiol 2019;20: 289-334.

4. Mennuni M, Gulizia MM, et al. Position paper ANMCO: Gestione della dimissione ospedaliera. G Ital Cardiol 2016;17:657-86. 


\title{
Modelli a confronto nei percorsi gestionali dello scompenso cardiaco: l'esperienza dell'ASST Rhodense
}

\author{
Sergio A. Berra \\ Medicina Interna, Garbagnate Milanese, ASST Rhodense (MI), Italia
}

\section{Introduzione}

Lo scompenso cardiaco riconosce una elevata incidenza nella popolazione generale, ne sono affetti in Italia più di 1 milione di pazienti e 15 milioni circa in Europa ed è la terza causa di ricovero ospedaliero. Stratificando per decadi di età si osserva poi una prevalenza di circa il $10 \%$ oltre i 70 aa, in Italia si stima che nel 2050 l'aspettativa di vita per i 65enni sarà prolungata di circa 30 aa. e noi sappiamo che l'insuff. cardiaca è predittrice di mortalità nella popolazione anziana e grande anziana. ${ }^{1}$ Lo scompenso cardiaco è inoltre causa di molteplici accessi in Pronto Soccorso, frequenti re-ricoveri ed il dato di mortalità è direttamente correlato con il numero degli stessi: il $25 \%$ circa muore entro un anno dalla diagnosi e oltre il 50\% entro i 5 anni. ${ }^{2,3}$ Modelli di gestione della patologia, simili ad altri per malattie croniche disegnano traiettorie di efficace gestione integrata coinvolgenti plurime figure in grado di rispondere tempestivamente e prospetticamente ai bisogni dell'assistito. ${ }^{4}$ Nuove tecnologie, interdisciplinarietà ed integrazione territoriale descrivono un futuro ormai accessibile e al quale la nostra Unità Operativa ha dedicato un progetto di presa in carico e follow up del paziente affetto da insuff. cardiaca di grado moderato e severo.

\section{Materiali e Metodi}

Il progetto ha ricercato soluzioni su una area ri-

Corrispondente: Sergio A. Berra, Medicina Interna, ASST Rhodense, Viale Forlanini 95, 20028 Garbagnate Milanese (MI), Italia.

E-mail: abiesse@libero.it

Articolo pubblicato secondo la Creative Commons Attribution NonCommercial 4.0 License (CC BY-NC 4.0).

${ }^{\circ}$ Copyright: the Author(s), 2021

Licensee PAGEPress, Italy

QUADERNI - Italian Journal of Medicine 2021; 9(2):e11 levante della patologia cardiovascolare nell'ambito della cronicità: l'insufficienza cardiaca, proponendo una semplificazione ed un miglioramento della rete territoriale di assistenza inserendosi nell'ormai collaudato sistema di telesorveglianza (progetto Nuove Reti Sanitarie di Regione Lombardia) della ASST Rhodense coordinato dalla Medicina Interna del presidio Ospedaliero di Garbagnate Milanese. ${ }^{1}$ Le azioni generali indicate cioè l'organizzazione di una rete “HUB\&SPOKE' inerente alla patologia assumono valenza ulteriore poiché propongono una pratica di collaborazione fra risorse sanitarie che si occupano delle stesse patologie in un sistema a rete integrata. La flessibilità del modello inoltre ha permesso un costante aggiornamento o modulazione dei nodi della rete, in base all'aggiornamento dei programmi terapeutico-assistenziali. ${ }^{5}$

Il disegno ha visto quindi l'organizzazione di Servizi afferenti alla stessa traiettoria di patologia, con interventi di coordinamento, sorveglianza e gestione burocratico/amministrativa (Hub presidio di Bollate) e terminali di accesso in centri sott'ordinati (Spoke presidi di Garbagnate - Rho - Passirana) a cui era riferito il reclutamento, la selezione e l'invio dei pazienti. Il continuum della malattia cardiovascolare ha riconosciuto quindi diversi momenti nel suo percorso: i) degenza ospedaliera (UU.OO. Medicina Interna UU.OO. Cardiologia - U.O. Medicina ad indirizzo geriatrico - Riabilitazione Cardiologica); ii) ambulatori per il follow up di interesse Internistico - Cardiologico - Riabilitativo; iii) reclutamento nel programma di telesorveglianza; iv) integrazione medici di medicina generale.

Il modello organizzativo proposto ha permesso da un lato un miglior utilizzo delle risorse sanitarie disponibili nei percorsi già in atto nei presidi di Rho, Garbagnate e Passirana e dall'altro un confronto tra le diverse realtà per l'identificazione di ulteriori Spoke, le varie fasi del progetto infine hanno obbligato comunque ad un ripensamento finalizzato degli investimenti con le risorse disponibili (necessità di 2 collaboratori sanitari - infermieri professionali - dedicati al coordinamento) (Figura 1). 


\section{Il modello adottato}

Alla dimissione era proposta l'adesione al percorso dal medico e dall'infermiere tutor, condiviso con il caregiver del paziente, ed in tale contesto erano consegnati il device di trasmissione del segnale elettrocardiografico, i numeri telefonici di riferimento e le istruzioni per l'autocontrollo della malattia al fine di riconoscere precocemente i segni di instabilizzazione emodinamica. ${ }^{6}$ I parametri monitorati al domicilio erano il peso, la pressione arteriosa, la frequenza cardiaca, la traccia elettrocardiografica, dati trasmessi settimanalmente all'infermiere tutor nell'ambito di una telefonata strutturata e programmata e compilati su cartella clinica elettronica consultabile anche da remoto nell'ambito del rispetto della normativa della privacy (Figura 2).

I percorsi erano suddivisi in alta intensità, della durata di 6 mesi, con trasmissione di segnale ECG, parametri vitali, e telefonata strutturata settimanale e bassa intensità che prevedeva invece solo una telefonata strutturata settimanale ed a necessità, per entrambi era indicata la compilazione della cartella clinica elettronica (Figura 2).
La monitorizzazione era aperta $24 / 24$, gestita nelle ore notturne, prefestive e festive da un provider e nelle ore diurne settimanali dal Centro Servizi Aziendale.

Eventuali segnali di criticità per instabilizzazione erano segnalati al medico ed il paziente era invitato precocemente all'ambulatorio scompenso o dirottato in Pronto Soccorso in relazione al quadro clinico, in collaborazione con il MMG (Figura 3).

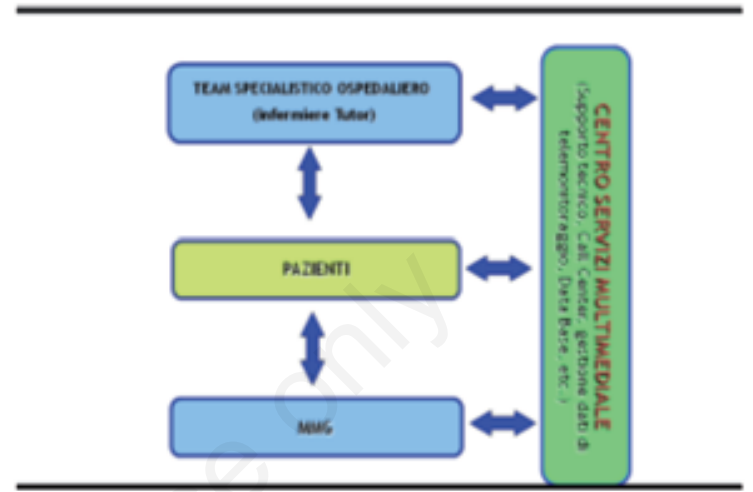

Figura 1. Flow chart organizzativa.

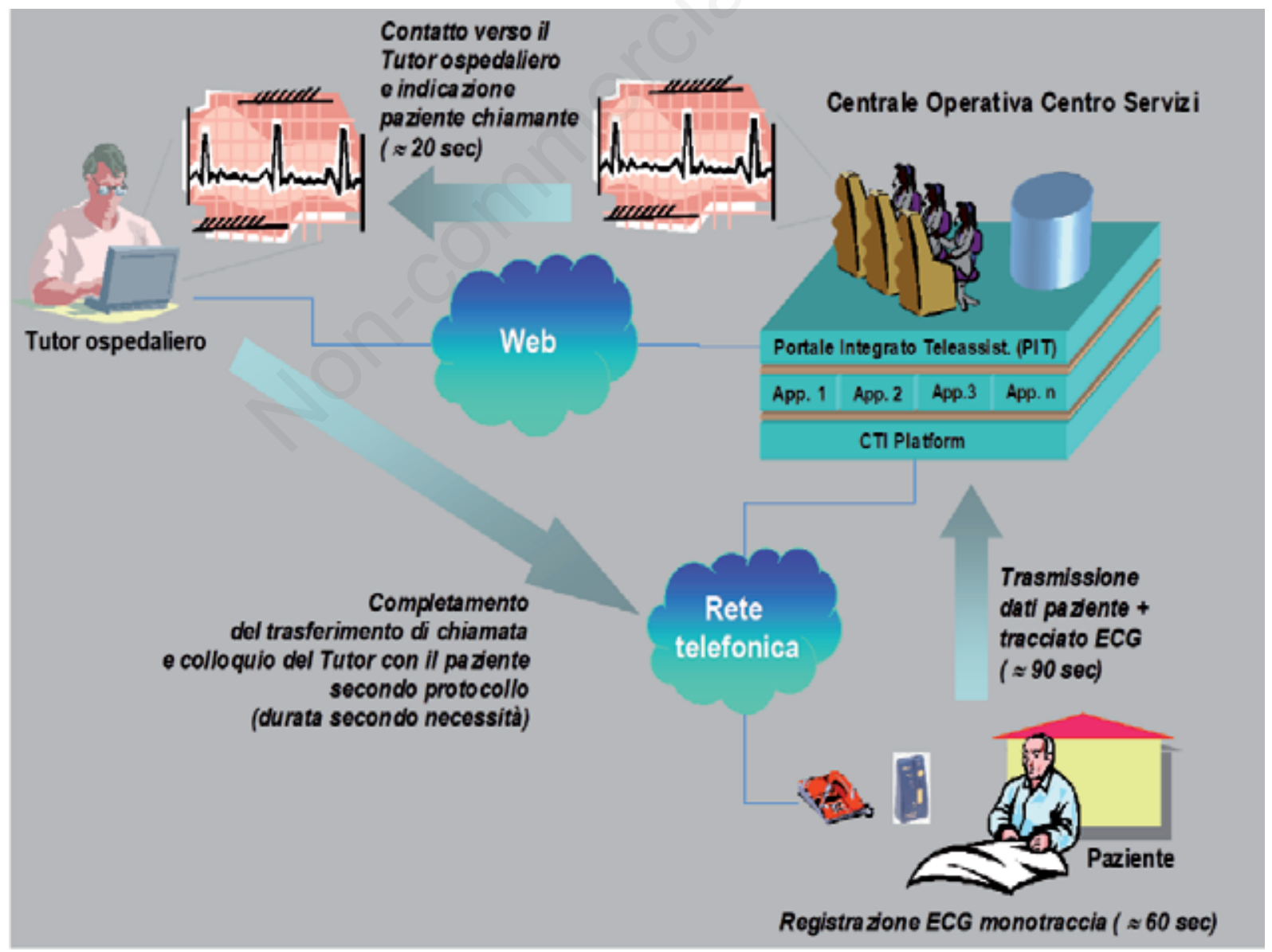

Figura 2. Invio del segnale ECG e della successiva intervista. 


\section{Risultati}

Nell'arco temporale 2007/2018 sono stati arruolati oltre 200 pazienti con benefici in termini di riduzione dei re-ricoveri (15-20\%), miglioramento della qualità di vita (QoLS) ed aderenza alla terapia. L'età media era di 80 aa (Figura 4); l'eziologia prevalente era la cardiopatia ischemica (Figura 5); la comorbidità prevalente era l'ipertensione arteriosa (Figura 6); la media dei contatti telefonici non programmati è stata di circa 11 nei sei mesi dell'arruolamento in Alta Intensità (Figura 7); infine le cause più frequenti di interruzione della telesorveglianza erano ricoveri per lo più per nuovi eventi cardiovascolari (Figura 8).

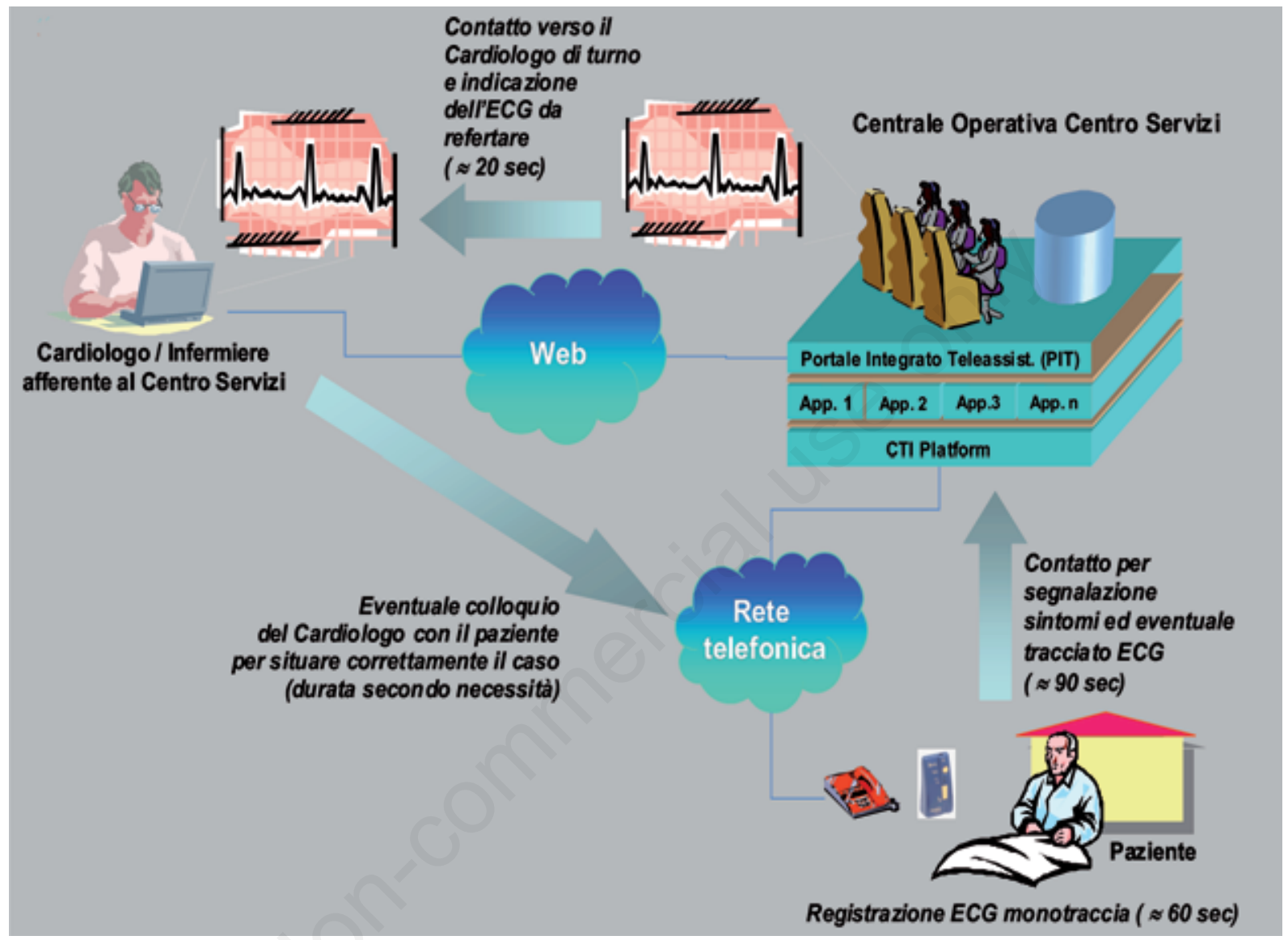

Figura 3. Contatto per comparsa di sintomo.

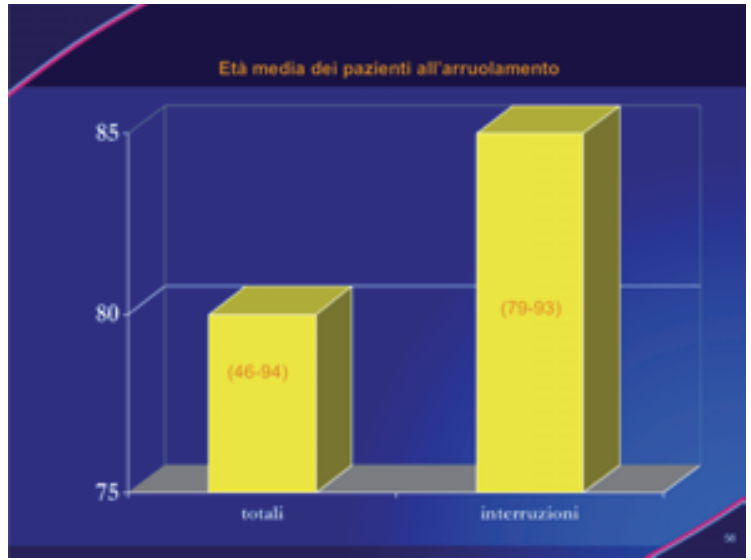

Figura 4. L'eta media era di 80 aa.

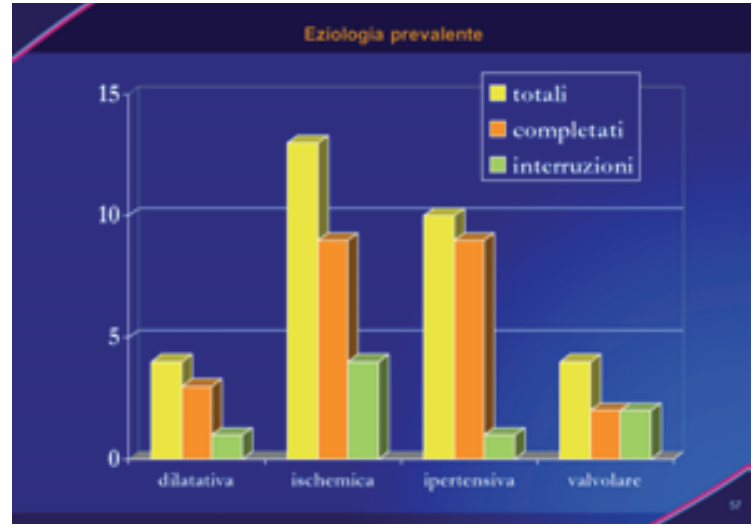

Figura 5. L'eziologia prevalente era la cardiopatia ischemica. 


\section{Conclusioni}

L'intervento integrato dello scompenso cardiaco prevede certamente la creazione di Unità per lo Scompenso Cardiaco sia ambulatoriali che ospedaliere con expertise di gestione a valenza internistica e cardiologica. ${ }^{6}$

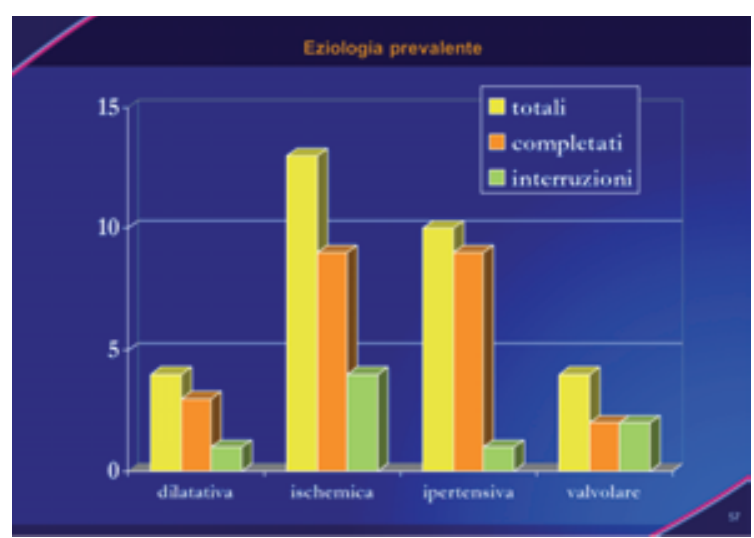

Figura 6. La comobidità prevalente era l'ipertensione arteriosa.

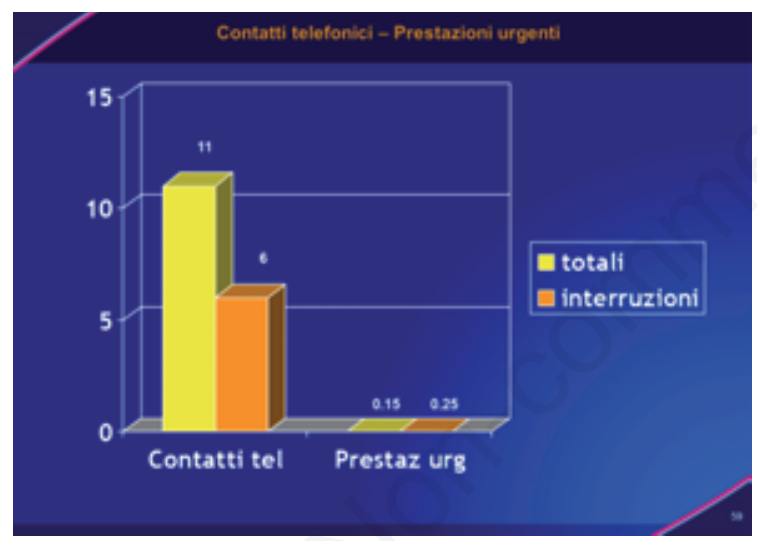

Figura 7. Contatti telefonici.

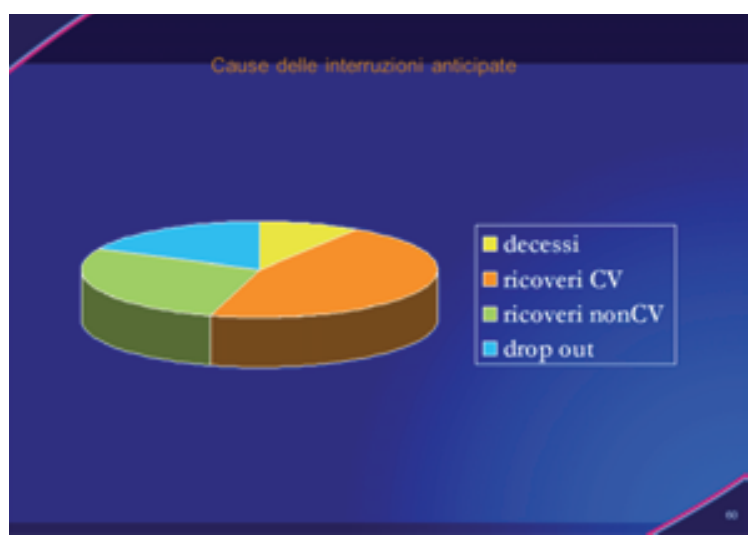

Figura 8. Drop Out.
La telemedicina offre una interazione tra vari attori per una patologia che necessariamente, in relazione anche alla sua evoluzione, deve avere risposte cliniche ed organizzative precoci e condivise.

Stime in letteratura indicano una riduzione del 30$35 \%$ della mortalità e del $15-20 \%$ delle ospedalizzazioni.

L'ambulatorio scompenso nella nostra organizzazione è stato implementato da un Day-Hospital intensivo per pazienti affetti da insufficienza cardiaca e da un progetto Empowerment nel paz. affetto da Scompenso Cardiaco che si avvale del contributo di un medico esperto, un fisioterapista, uno psicologo, un nutrizionista i quali ciascuno per il proprio ruolo assicurano la fidelizzazione dei pazienti e dei loro caregivers.

Il modello adottato, certamente più efficace, nei quadri moderati e gravi offre sicurezza al paziente per un interlocutore sempre disponibile avvicinando il setting assistenziale ad una forma di ospedalizzazione domiciliare.

L'attivazione di una presa in carico domiciliare del paziente affetto da insufficienza cardiaca apre una prospettiva di deospedalizzazione che affida a criteri di miglior sicurezza il loro destino consegnando loro un mandato che attraverso le nuove tecnologie permette una precoce diagnosi di instabilizzazione con una conseguente riduzione di mortalità ed ospedalizzazione ed un conseguente significativo risparmio di spesa.

'...Avrò in sorte di essere il più curato dei malati. Ma nessuno può superare i limiti prescritti dalla natura; le gambe gonfie non mi sostengono più nelle lunghe cerimonie di Roma; mi sento soffocare; ed ho sessant'anni' da Memorie di Adriano, M. Yourcenar

\section{Bibliografia}

1. Borghi G, Masella C, Zanaboni P, Bartoli L. Nuove Reti Sanitarie - Un servizio sperimentale della Regione Lombardia.

2. Benatar D., Bondmass M., Ghitelman J., Avitall B. (2003). "Outcomes of chronic heart failure", Archives of Internal Medicine, Vol. 163, No. 3, pp. 347-352 Berry C., Murdoch D.R., McMurray J.J. (2001) "Economics of chronic heart failure", Europ. Journal Hearth Failure, Vol. 3, No. 3, pp. 283-291.

3. Bundkirchen A, Schwinger RHG. Epidemiology and economic burden of chronic hear failure. Euro Heart J Suppl 2004;6:D57-60.

4. Cleland JG, Louis AA, Rigby AS, et al. Noninvasive home telemonitoring for patients with heart failure at high risk of recurrent admission and death. J Am Coll Cardiol 2005;45:1654-64.

5. Verdiani V, Panigada G, Fortini A, et al. The heart failure in internal medicine in Tuscany: the SMIT Study. Ital J Med 2015;9:349-55.

6. Mortara A, Oliva F, Di Lenarda A. Prospettive della telemedicina e del monitoraggio mediante dispositivi nel paziente con scompenso cardiaco cronico: luci e ombre. G Ital Cardiol 2010;11:335-75. 


\title{
Modelli a confronto nei percorsi gestionali dello scompenso cardiaco: l'esperienza di Palmanova
}

\author{
Claudia Battello \\ Responsabile di SOS “Gestione Percorsi Clinici” della SOC di Medicina Interna di Palmanova, Azienda Sanitaria Universita- \\ ria Friuli Centrale, Udine, Italia
}

L'ambulatorio internistico dello Scompenso cardiaco presso l'Ospedale di Palmanova, attualmente presidio spoke dell'Azienda sanitaria del Friuli Centrale (Hub l'Ospedale di Udine), è stato avviato nel maggio 2017 quando il presidio faceva parte dell'allora Azienda Ospedaliera Bassa Friulana- Isontina, composta da 4 ospedali spoke con riferimento agli Hub di Udine (per la Bassa Friulana) e Trieste (per l'Isontino). Un gruppo di lavoro composto da infermieri, MMG, medici cardiologi ed internisti, sia ospedalieri che dei servizi territoriali, aveva redato un PDTA per cercare di uniformare i comportamenti dei singoli professionisti operanti in realtà territoriali molto diverse: tale PDTA è stato mantenuto dopo la riorganizzazione aziendale regionale.

Il percorso prevede la presa in carico dei pazienti ricoverati nel reparto di Medicina Interna per scompenso cardiaco acuto o per patologie ad esso correlate (ad esempio progressione dell'insufficienza renale, anemizzazione) ed un successivo follow-up ambulatoriale o territoriale.

All'ingresso il paziente viene inquadrato ed impostato come da linee guida internazionali: ${ }^{1}$ il paziente critico viene sottoposto a valutazione cardiologica (Servizio Cardiologico Aziendale) nel corso della degenza, mentre il paziente senza criticità, se si tratta di una prima diagnosi di scompenso, viene rinviato a valutazione cardiologica nelle settimane successive alla dimissione (Figura 1). Il paziente cronico viene abitualmente preso in carico

Corrispondente: Claudia Battello, Responsabile di SOS "Gestione Percorsi Clinici” della SOC di Medicina Interna di Palmanova, Azienda Sanitaria Universitaria Friuli Centrale, Udine, Italia.

E-mail: claudia.battello@asufc.sanita.fvg.it

Articolo pubblicato secondo la Creative Commons Attribution NonCommercial 4.0 License (CC BY-NC 4.0).

${ }^{\circ}$ Copyright: the Author(s), 2021

Licensee PAGEPress, Italy

QUADERNI - Italian Journal of Medicine 2021; 9(2):e12
dall'Internista per il follow-up ambulatoriale ed una visita cardiologica viene richiesta solo se l'evoluzione clinica richiede accertamenti e procedure specialistiche (impianto di pace-maker o defibrillatore, indicazione a risincronizzazione cardiaca, ecostress, studio coronarografico o scintigrafico) che possono essere erogati in sede, dal servizio Cardiologico, o presso l'ospedale Hub; se il paziente cronico risulta particolarmente fragile, viene segnalato ai servizi territoriali i cui infermieri, periodicamente formati, lo seguono a domicilio con la rilevazione dei parametri, il rinforzo educativo, la valutazione della compliance terapeutica.

Nello sviluppo di questo progetto a livello di SOC di Medicina di Palmanova, abbiamo focalizzato l'attenzione su due punti: i) l'educazione del paziente e dei caregivers; ii) la correzione della sideropenia, in collaborazione con l'ambulatorio Internisticoematologico presente nella struttura.

$\mathrm{Al}$ momento dell'ingresso, la definizione del piano di cura prevede la segnalazione del paziente alle Infermiere di continuità assistenziale (ICA) che si occupano degli aspetti educativi sia del paziente che dei familiari o altri caregivers (Figura 2); viene in particolare stressata l'attenzione alla restrizione idrica ed al controllo giornaliero del peso; inoltre nei giorni precedenti la dimissione il paziente e/o i familiari vengono educati alla modulazione della terapia diuretica orale. A pochi giorni dalla dimissione il paziente e/o i caregivers vengono contattati telefonicamente dalle ICA per un rinforzo educativo, la valutazione di alcuni parametri semplici come il peso, la pressione e la frequenza cardiaca ed il numero dei cuscini utilizzati a letto, parametri che orientano sull'andamento del compenso cardiocircolatorio e l'aderenza terapeutica del paziente; la telefonata viene ripetuta a circa 2 settimane, prima della visita programmata entro i $20 \mathrm{gg}$ dalla dimissione.

Tale metodo viene adottato sia dopo la prima che dopo le successive viste ambulatoriali, in particolare se viene consigliata una variazione terapeutica, per una valutazione dell'aderenza e dei benefici clinici; ad esempio viene utilizzato per la valutazione della risposta terapeutica alla variazione posologica del 
diuretico, qualora il paziente segnali telefonicamente un repentino incremento ponderale o una contrazione della diuresi, o per valutare la risposta all'up-grading del $\beta$-bloccante e degli ace-inibitori/sartani.

Anche i Servizi territoriali utilizzano il contatto telefonico per la valutazione dei parametri e l'aderenza terapeutica sia a pochi gg dalla dimissione che dopo le prime visite domiciliari; il rilievo di progressivi segni di scompenso, come un peggioramento della dispnea e un incremento degli edemi declivi, induce una piccola variazione posologica del diuretico da parte dell'infermiere ed attiva la visita domiciliare da parte del MMG.

Ad ogni contatto telefonico corrisponde la compilazione di un modulo prestampato riportante i parametri rilevati e le annotazioni sulle variazioni di terapia; il modulo viene conservato nel fascicolo sanitario del paziente sia esso ambulatoriale che del territorio.
Come risultato di progetto, nonostante i piccoli numeri ancora a disposizione, abbiamo osservato: i) l'enpowerment educativo dei pazienti e loro familiari; ii) una maggior integrazione ospedale-territorio; iii) una riduzione dei riaccessi ospedalieri dei pazienti presi in carico.

All'ingresso in reparto tutti i pazienti con diagnosi di scompenso cardiaco o patologie ad esso correlate, eseguono, con gli esami di routine (comprendente l'emocromo), anche uno studio anemia (Figura 3), ossia il dosaggio sierico della ferritina, ferro e transferrina: viene così definito l'eventuale stato di anemia (emoglobina $[\mathrm{Hb}]<130$ $\mathrm{g} / \mathrm{L}$ nel maschio ed $\mathrm{Hb}<120 \mathrm{~g} / \mathrm{L}$ nella femmina) o di sideropenia (ferritina $<100 \mathrm{ng} / \mathrm{mL}$ o ferritina tra 100 $299 \mathrm{ng} / \mathrm{mL}$ e saturazione della transferrina $<20 \%)^{2,3}$ Il calcolo della saturazione della transferrina (TSAT $=$ sideremia/ [transferrina x 1,42$] \times 100)$ consente di
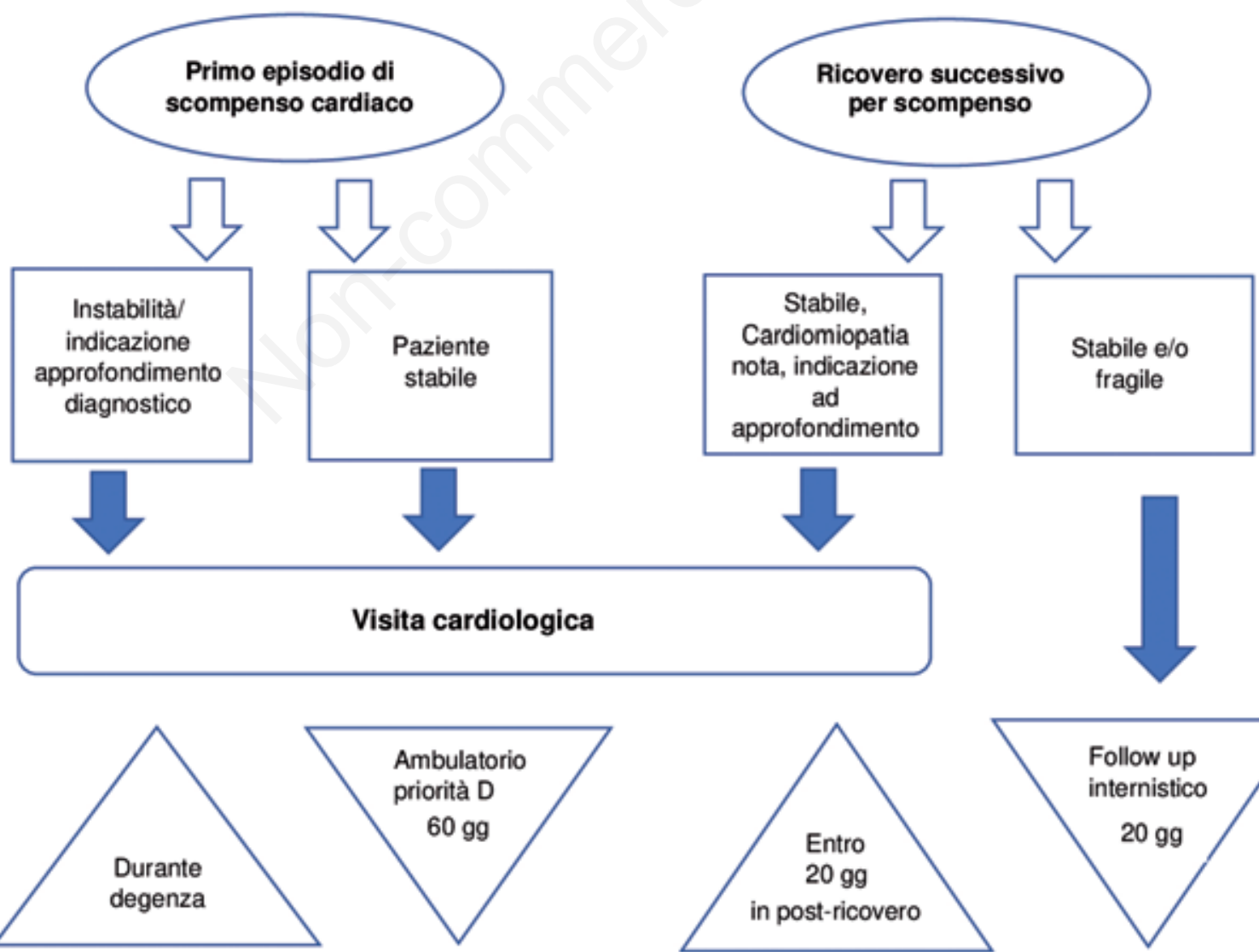

Figura 1. Percorso del paziente ricoverato per scompenso cardiaco. 
rilevare una carenza marziale anche nei pazienti con ferritina aumentata come risposta ad un quadro flogistico. Nonostante i dati di letteratura evidenzino come la causa principale di sideropenia nello scompenso cardiaco sia legata alla flogosi cronica che caratterizza la sindrome o abbia una genesi multifattoriale (carenze alimentari, malassorbimento, uso di farmaci, insufficienza renale cronica, etc.), i pazienti sideropenici vengono screenati per le perdite croniche gastrointestinali ed eventualmente sottoposti ad esami endoscopici. Per la definizione dell'entità della supplementazione marziale con ferro carbossimaltoso (FCM) ev viene applicata la formula di Ganzoni (peso [kg] x $\{\mathrm{Hb}$ target [150] $-\mathrm{Hb}$ attuale $[\mathrm{g} / \mathrm{L}]\}$ x 0,24 + 500): una dose massima di FCM di
$1000 \mathrm{mg}$ viene somministrata in corso di degenza, e qualora non fosse sufficiente, viene programmata una seconda infusione a distanza di almeno una settimana $\mathrm{o}$ in regime di ricovero $\mathrm{o}$, più frequentemente, ambulatorialmente nel corso della prima visita di follow-up. Successivamente viene richiesto un monitoraggio dell'emocromo a 2, 4, 12, 24 e 52 settimane dall'ultima infusione ed il miglioramento funzionale viene valutato con il test del cammino dei 6 minuti al tempo zero, a 12 e 24 settimane.

Fino ad ora il $93 \%$ dei pazienti trattati ambulatorialmente non ha presentato ricoveri successivi per riacutizzazione dello scompenso cardiaco, ma la numerosità del campione non è ancora sufficiente per una analisi statistica.

\section{Enpowerment educativo:}

ruolo dell'infermiera di continuità assistenziale e delle infermiere territoriali
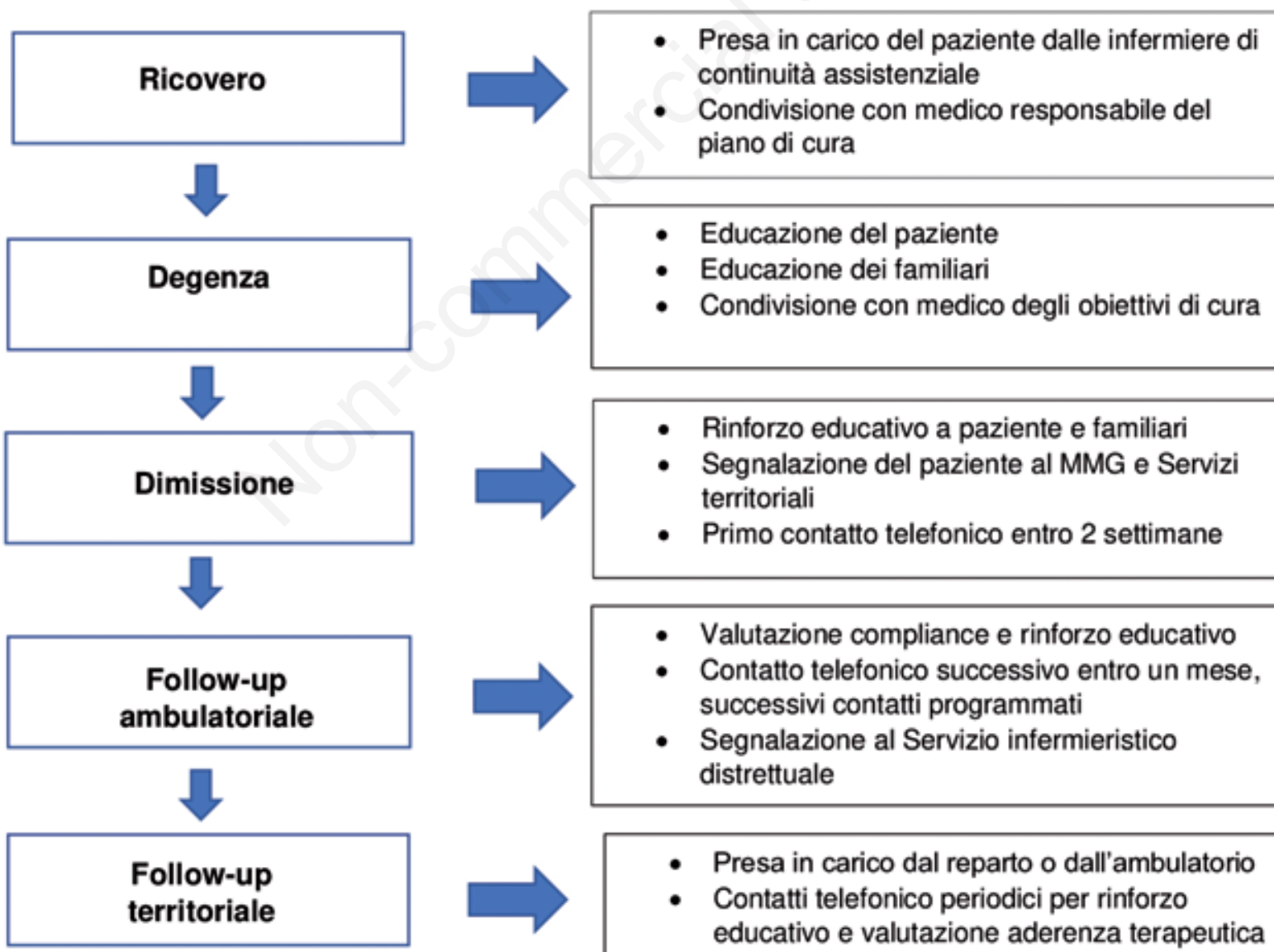

- Educazione del paziente

- Educazione dei familiari

- Condivisione con medico degli obiettivi di cura

- Rinforzo educativo a paziente e familiari

- Segnalazione del paziente al MMG e Servizi territoriali

- Primo contatto telefonico entro 2 settimane
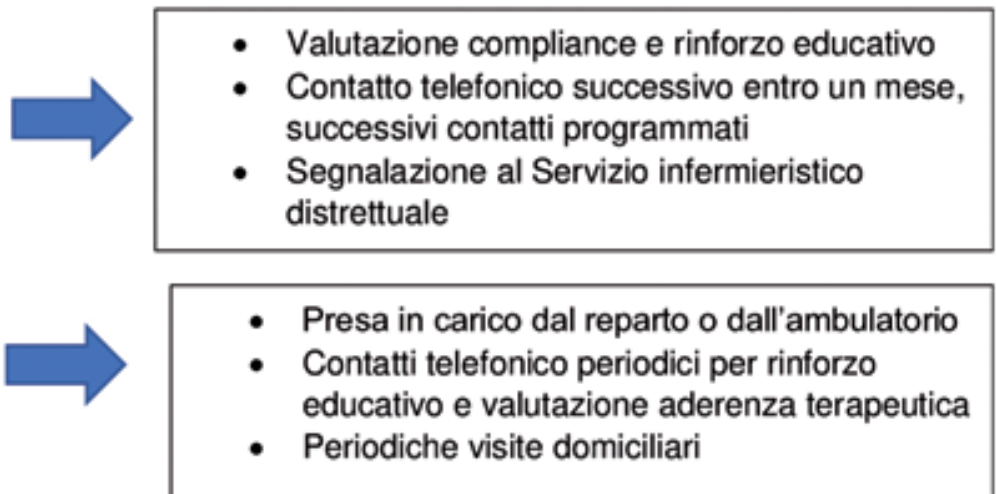

Figura 2. Interventi degli infermieri di continuità assistenziale e degli infermieri territoriali nel follow-up del paziente con scompenso cardiaco cronico. 


\section{Percorso scompenso cardiaco e sideropenia}

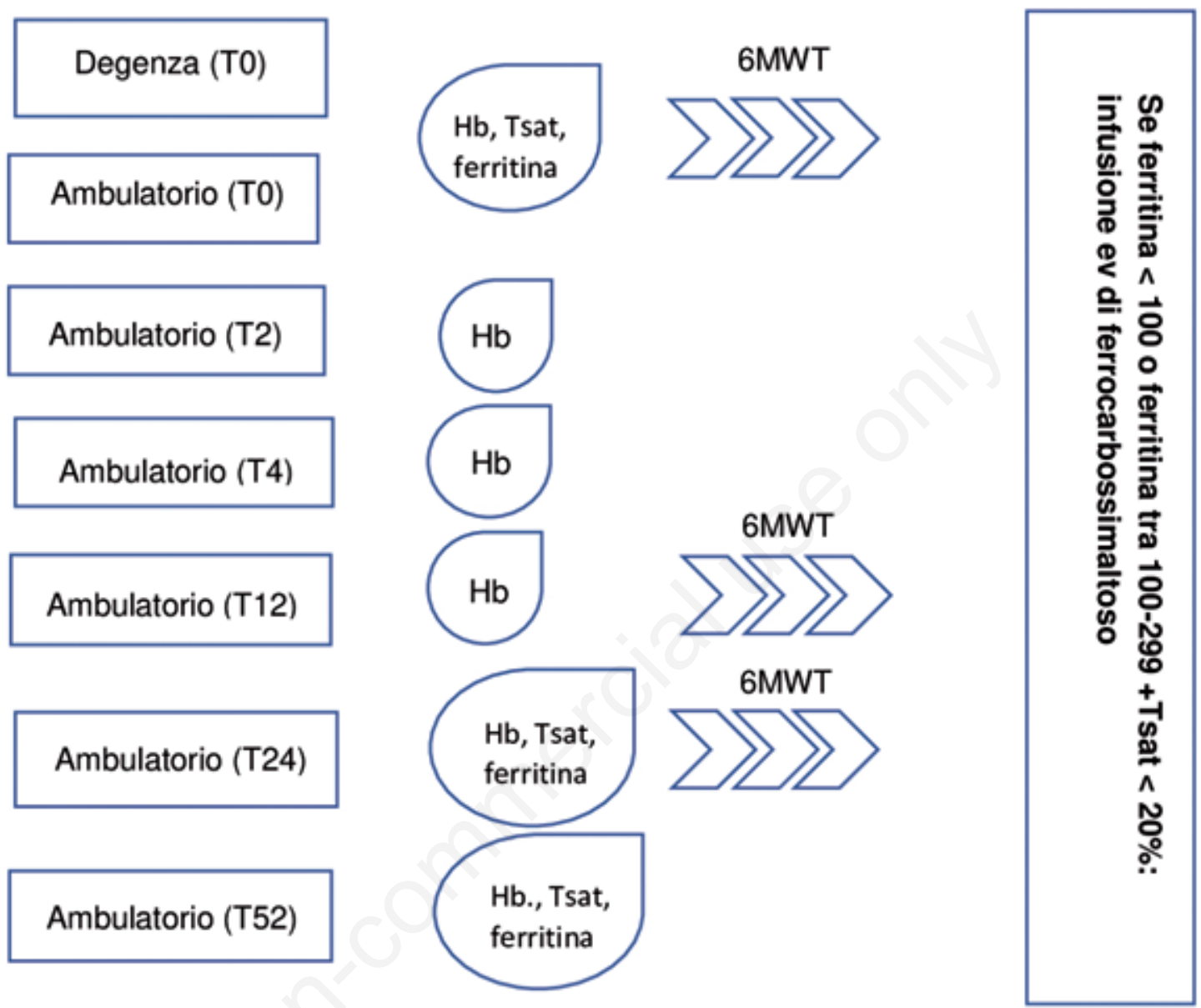

Figura 3. Monitoraggio della sideropenia e/o anemia nel follow-up ambulatoriale.

\section{Bibliografia}

1. European Society of Cardiology. Acute and Chronic Heart Failure Guidelines. EHJ 2016;37: 2129-200.

2. Ricciardi E, La Malfa G, Guglielmi G, et al. Characteristics of current heart failure patients admitted to internal medicine vs. cardiology hospital units: the VASCO study. Intern Emerg Med 2020, 14 March [Epub ahead of print].

3. Gonzalez-Costello J, Cainzos-Achirica M, Lupon J, et al. Use of intravenous iron in patients with iron deficiency and chronic heart failure: real-word evidence. EJIM https://doi.org/10.1016/j.ejim.2020.04.031 


\title{
Modelli a confronto nei percorsi gestionali dello scompenso cardiaco: l'esperienza dell'AUSL di Bologna
}

\author{
Stefano Urbinati, ${ }^{1}$ Maurizio Ongari ${ }^{2}$ \\ ${ }^{1}$ UOC Cardiologia, Ospedale Bellaria, AUSL Bologna; ${ }^{2}$ UOC Medicina Interna, Ospedale Porretta Terme, AUSL Bologna, Italia
}

\section{Gli inizi e la fase degli audit}

L'esigenza di standardizzare la gestione dello scompenso cardiaco a Bologna si sviluppa nei primi anni ' 90 quando fu realizzato un primo progetto, chiamato Oscar, che prevedeva soprattutto una sincronizzazione culturale, infatti venti anni fa il mondo cardiologico e quello internistico non erano ancora ancora consapevoli dell'impatto che lo scompenso cardiaco avrebbe avuto sull'epidemiologia delle malattie cardiovascolari. A metà degli anni ' 00 è stato formalizzato un secondo progetto, chiamato BO.SCO. cioè Bologna Scompenso, che ha cercato di definire la situazione sul piano epidemiologico, sviluppando progetti pilota come gli audit annuali sulle lettere di dimissione e una sperimentazione di integrazione tra ospedale e territorio effettuata a livello di un distretto territoriale, quello di San Lazzaro, in cui è stata avviata una gestione in team tra la Cardiologia ospedaliera di riferimento, la Cardiologia territoriale e i tre Nuclei di Cure Primarie. L'audit sulle lettere di dimissione, effettuato annualmente tra il 2006 e il 2013, ha fatto emergere alcune importanti criticità come la difficoltà, da parte dei reparti internistici o geriatrici, di approfondire le indagini per definire l'eziologia, indentificare il fattore precipitante ed effettuare la stratificazione prognostica, anche a causa di un non facile accesso all'ecocardiografia, e la mancanza di un percorso strutturato di follow-up dopo la dimissione. In quegli anni è stato condiviso che la lettera di dimissione costituisca lo strumento centrale per il passaggio delle consegne tra il ricovero ospedaliero e la gestione post-dimissione.

Nel 2016 l'AUSL di Bologna ha deciso che era arrivato il momento di sistematizzare la gestione dello

Corrispondente: Stefano Urbinati, UOC Cardiologia, Ospedale Bellaria, AUSL Bologna, Italia.

E-mail: stefano.urbinati@ausl.bologna.it

Articolo pubblicato secondo la Creative Commons Attribution NonCommercial 4.0 License (CC BY-NC 4.0).

${ }^{\circ}$ Copyright: the Author(s), 2021

Licensee PAGEPress, Italy

QUADERNI - Italian Journal of Medicine 2021; 9(2):e13 scompenso cardiaco a livello di tutta l'Azienda deliberando il PDTA scompenso cardiaco coordinato da un clinico e da un referente assistenziale con un importante supporto da parte del governo clinico. La scelta di gestire lo scompenso cardiaco con uno strumento come il PDTA è dovuto alla sua elevata prevalenza, all'alto tasso di disabilità, all'importante assorbimento di risorse, a causa delle frequenti ospedalizzazioni, e ai numerosi attori coinvolti. In questo senso la costituzione di un gruppo di lavoro per il governo del PDTA va considerata come una grande opportunità di learning by doing e di monitorare quasi in tempo reale la situazione con la possibilità di apportare tempestivamente dei cambiamenti in base all'evoluzione dei bisogni.

\section{Il modello organizzativo attuale}

Dopo il primo triennio, in cui si è cercato soprattutto di mettere a sistema l'offerta esistente, nel corso del 2019 è stato costituito un tavolo interaziendale (con l'AOSP) su PDTA, appropriatezza ed umanizzazione che ha portato alla ridefinizione del modello organizzativo e all'introduzione di alcune novità importanti come la stratificazione prognostica, la differenziazione dell'offerta a livello territoriale e il completamento dell'offerta con il coinvolgimento del Centro Trapianti e il Centro per le Cardiomiopatie. Un' analisi ha dimostrato che i pazienti inseriti nel percorso presentano un minore ricorso ai PS e meno ospedalizzazioni, a fronte di un maggior numero di visite cardiologiche, mentre un focus group sui caregivers ha fatto emergere bisogni nuovi che devono fare parte integrante del percorso. Il PDTA scompenso cardiaco, revisionato nel 2019, è stato inserito nell'accreditamento regionale della AUSL di Bologna. ${ }^{1-4}$

\section{I percorsi post-dimissione}

L'area metropolitana di Bologna ha una popolazione di oltre 850.000 persone, residenti in oltre 50 comuni, con alta prevalenza di anziani (il $24 \%$ ha un'età $>65$ anni e il $13 \%$ un'età $>75$ anni) di cui circa la metà vivono da soli. Dal punto di vista sanitario il territorio è articolato in 6 distretti, e quello di Bologna-città in 5 
quartieri, sono presenti 9 ospedali dell'AUSL e il policlinico che identifica l'AOSP. Nei distretti sono presenti le Case della Salute, che devono essere considerate delle strutture, non solo logistiche, dove sono coordinate e concentrate le attività territoriali.

Dal punto di vista epidemiologico circa il 15\% dei pazienti che accedono al PS/MEU con un quadro di scompenso cardiaco viene dimesso direttamente a domicilio. Tra quelli che vengono ricoverati $1{ }^{\prime} 85 \%$ dei pazienti ha accesso ad un reparto internistico o geriatrico, mentre il $15 \%$ viene ricoverato in cardiologia. Nel 2019 i pazienti ricoverati per scompenso cardiaco nell'area metropolitana di Bologna sono stati 2535, con un calo del $16,3 \%$ dei ricoveri rispetto al 2014 quando erano stati 3032. L'effettuazione di una visita cardiologica post-dimissione entro 90 giorni, che si è dimostrata predittiva di prognosi migliore, è stata effettuata nel $45,3 \%$, con un significativo incremento rispetto al 2017 quando era stata effettuata solo nel $29,6 \%$. È diminuita la mortalità a 30 giorni che è stata del $4,7 \%$ contro il $7 \%$ del 2017 , mentre non è diminuito in maniera significativa il numero delle riospedalizzazioni a 15, 30 e 60 giorni che si sono osservate rispettivamente nell' $8,5 \%, 15,3 \%$ e $24,3 \%$ dei casi contro $1^{\prime} 8.9 \%, 14,2 \%$ e $23,2 \%$ del 2017.

Il modello organizzativo messo a punto nel corso del 2019, che prevede l'entrata preferenziale nel PDTA dei pazienti dimessi, ha definito tre percorsi differenti, a seconda che prevalessero criticità di tipo cardiologico, internistico o socio-sanitario. I percorsi sono attivati alla dimissione in maniera informatica comunicando al medico di medicina generale e ai servizi territoriali.

Il percorso prevalentemente cardiologico riguarda pazienti con scompenso cardiaco de novo, con disfunzione ventricolare sinistra, valvulopatie severe, fibrillazione atriale con frequenza cardiaca non controllata, cuore polmonare cronico o insufficienza tricuspidalica severa che devono affrontare snodi decisionali cardiologici (soprattutto relativi alla necessità di essere sottoposti a procedure interventistiche) o titolazione della terapia. Questo percorso prevede una presa in carico da parte della cardiologia ospedaliera di riferimento (alla dimissione deve essere prenotata una visita cardiologica di controllo post-dimissione presso l'ambulatorio dedicato) che non esclude, se utile, una concomitante presa in carico territoriale medico-infermieristica.

Il percorso prevalentemente internistico riguarda la maggior parte dei pazienti, in cui prevalgono le comorbidità e le problematiche internistico-geriatriche. La gestione territoriale dovrebbe iniziare con una dimissione protetta e proseguire mediante la realizzazione di un piano assistenziale individualizzato integrato (PAII), condiviso tra medico di medicina generale ed equipe infermieristica territoriale o domiciliare, orientato a dare risposta alle priorità (eventualmente suggerite nella lettera di dimissione) sulle quali focalizzare il monitoraggio e gli interventi. In questo caso è previsto un back-up cardiologico da parte della cardiologia territoriale che ha destinato una parte della sua offerta a questa finalità.

Infine il percorso prevalentemente socio-sanitario si riferisce a pazienti di età molto avanzata, che vivono soli oppure con severo decadimento cognitivo, non autosufficienti o con grave deprivazione sociale. Questo percorso prevede che i pazienti siano segnalati già in fase di ricovero al Servizio di Assistenza Socio-Sanitaria e che successivamente siano presi in carico dai servizi territoriali che definiranno, insieme alla famiglia, il percorso più adeguato, considerando anche la possibilità che il paziente che non presenta le condizioni per vivere a domicilio possa essere affidato, in maniera temporanea o definitiva, a strutture dedicate.

È importante sottolineare come questi tre percorsi siano diversamente declinati nelle tre principali aree geografiche dell'area metropolitana che sono quelle di Bologna Città, Pianura e Appennino che hanno offerte, organizzazione e criticità differenti.

Negli ultimi anni la migliore conoscenza del PDTA ha determinato un aumento significativo delle segnalazioni effettuate alla dimissione che sono passate dalle 803 del 2017 alle oltre 1000 del 2019. A questi pazienti vanno aggiunti i circa 800 pazienti già in carico agli ambulatori cardiologici dedicati allo scompenso cardiaco a livello ospedaliero. Invece è tuttora difficile censire quanti siano i casi inquadrabili come di pertinenza socio-sanitaria, tipologia di assistenza che esula dagli obiettivi del PDTA e che è tradizionalmente buona e distribuita in maniera capillare nell'area metropolitana. Un punto debole della nostra organizzazione è tuttora rappresentato dall'inadeguata informatizzazione che non permette di seguire il paziente nel suo percorso, né di monitorare in maniera costante gli indicatori.

\section{Gli obiettivi che ci eravamo dati per il 2020 e l'avvento della pandemia}

Per il 2020 erano state previste una serie di azioni che andavano dal potenziamento dell'assistenza territoriale, con un progetto specifico finalizzato ai caregivers, alla revisione degli indicatori, dall'implementazione dell'informatizzazione, alla definizione di una collaborazione più stretta con l'AOSP e con gli ospedali del privato accreditato, dalla formazione per i medici di medicina generale, anche in considerazione dell'ampio avvicendamento, alla sperimentazione sulla dimissione breve (fast track) dal PS/MEU. Inoltre, nello sviluppo della nuova CCE (cartella clinica elettronica) presso la AUSL di Bologna è in corso di identificazione di un minimal data set di indicatori che identifichino correttamente i pazienti con 
scompenso cardiaco e che possa essere utilizzato durante la gestione post-dimissione.

Purtroppo molte di queste azioni sono state bloccate o ritardate dall'avvento della pandemia. La disponibilità di visite cardiologiche territoriali e le visite internistiche precoci post-dimissione sono state sacrificate alle esigenze dell'emergenza COVID-19. Dal punto di vista pratico durante il lockdown, cioè nel trimestre marzo-maggio, abbiamo rilevato una riduzione dei ricoveri per scompenso cardiaco nell'ordine del $10-15 \%$, con un incremento significativo della mortalità che è tornata sopra il 7\%. Inoltre abbiamo osservato anche un incremento delle riospedalizzazioni.

Durante il lockdown è stata sviluppata la gestione da remoto basata sulle telefonate: in molti casi è stato fornito un numero verde oppure è stata condotta un'assistenza proattiva nei confronti dei pazienti più fragili. In questo modo si è limitato l'accesso all'ospedale alle urgenze. Alla riapertura delle attività, avvenuta tra maggio e giugno, si è constatato che fortunatamente il periodo di lockdown, nella maggior parte dei casi, era stato sufficientemente breve da non aver compromesso la qualità dell'assistenza. La letteratura recente sottolinea come, nel corso della pandemia, i pazienti con scompenso cardiaco contagiati abbiano presentato una mortalità più elevata, anche se non abbiamo ancora dati definitivi al riguardo.

Purtroppo nel periodo giugno-settembre a causa della contrazione dei posti-letto post-COVID e della riorganizzazione in corso il numero delle segnalazioni e delle prese in carico territoriali si sono significativamente ridotte, lontane dai livelli del 2019.

\section{Convivere con la pandemia e promuovere un nuovo piano per la cronicità}

Nell'ottobre 2020, mentre stiamo scrivendo questo capitolo, l'aumento esponenziale dei contagi da COVID-19, che configura la temuta seconda ondata, rende necessaria l'elaborazione di un piano alternativo che salvaguardi l'assistenza i pazienti con scompenso cardiaco in un nuovo periodo critico che andrà a sovrapporsi a quello dell'epidemia influenzale stagionale. Sulla base dell'esperienza maturata nella prima fase della pandemia sono state formulate alcune proposte per la gestione nei prossimi mesi:

- Istituzione di un database dei pazienti in carico dove siano riportate le criticità del singolo soggetto per la promozione di un'assistenza proattiva. È fondamentale che il database consenta un tracciamento del paziente e che venga integrato con il suo fascicolo sanitario.

- Dimissione fast-track dal PS/MEU/OBI per i pazienti già in carico con la collaborazione dell'infer- miere di continuità e dei servizi territoriali. Secondo alcune esperienze tale percentuale può passare dal $15 \%$ attuale fino al $35-40 \%$, ma per raggiungere tali risultati è necessario disporre di un database che permetta una loro rapida identificazione.

- Implementazione di forme di telemedicina con sistema di videochiamata che permettano la gestione clinico-infermieristica a distanza, nei casi in cui non sia possibile o non sia opportuno, effettuare la valutazione in presenza. Nel caso specifico l'obiettivo è quello di garantire un adeguato monitoraggio dei sintomi e dei parametri autogestiti dal paziente e dal caregiver (PA, FC, saturazione 02 , etc.).

- Adozione urgente di tele-trasmissione degli ECG e videochiamata dalle CRA. Nell'area di Bologna sono presenti 57 CRA che accolgono circa più di 4000 ospiti. Come è noto gli ospiti delle CRA, specialmente in un periodo di epidemia, è opportuno che non si spostino perché ad alto rischio di infezione e di letalità, perciò la gestione da remoto di questi pazienti fragili deve essere garantita rapidamente.

- Verifica dell'aderenza alla terapia farmacologica e allo stile di vita. Quando i pazienti vivono da soli, come avviene in una percentuale importante di casi, anche quelli autosufficienti diventano estremamente vulnerabili, perché spesso sottovalutano o non danno il giusto peso ad eventuali sintomi sentinella e, in caso di peggioramento urgente, non vengono soccorsi tempestivamente. È quindi importante che nell'ambito del database venga enfatizzato questo sottogruppo e, insieme con il medico di medicina generale e i servizi territoriali, si definisca un progetto individualizzato per la loro sorveglianza.

\section{Bibliografia}

1. Avaldi VM, Lenzi J, Urbinati S, et al. Effect of cardiologist care on 6-month outcomes in patients discharged with heart failure: results from an observational study based on administrative data. BMJ Open 2017;7: e018243.

2. Lenzi J, Avaldi VM, Hernandez-Boussard T, et al. Riskadjustment models for heart failure patients' 30-day mortality and readmission rates: the incremental value of clinical data abstracted from medical charts beyond hospital discharge record. BMC Health Serv Res 2016;16:473.

3. Avaldi VM, Lenzi J, Castaldini I, et al. Hospital readmissions of patients with heart failure: the impact of hospital and primary care organizational factors in Northern Italy. PLoS One 2015;10:e127796.

4. Lenzi J, Avaldi VM, Molinazzi D, et al. Are degree of urbanisation and travel times to healthcare services associated with the processes of care and outcomes of heart failure? A retrospective cohort study based on administrative data. PLoS One 2019;14:e0223845. 


\title{
Modelli a confronto nei percorsi gestionali dello scompenso cardiaco: l'esperienza delle Aziende USL della Toscana
}

\author{
Alberto Fortini, ${ }^{1}$ Giancarlo Tintori, ${ }^{2}$ Massimo Alessandri ${ }^{3}$ \\ ${ }^{1}$ Medicina Interna, Ospedale San Giovanni di Dio di Firenze, Azienda USL Toscana Centro; ${ }^{2}$ Medicina Interna, Ospedale Santa \\ Croce di Castelnuovo Garfagnana (LU) e Ospedale San Francesco di Barga (LU), Azienda USL Toscana Nord-Ovest; ${ }^{3}$ Medicina \\ Interna, Ospedale Sant'Andrea di Massa Marittima (GR), Azienda USL Toscana Sud-Est, Italia
}

\section{Introduzione}

Per quanto riguarda il Servizio Sanitario la Regione Toscana, all'interno di ognuna dei tre territori di Area Vasta (AV) in cui è suddivisa, e cioè l'AV Centro, l'AV Nord-Ovest e l'AV Sud-Est, vede operare, dal $1^{\circ}$ febbraio 2016, sia una Azienda Usl che una Azienda Ospedaliero-Universitaria. A queste sei Aziende si aggiunge l'Azienda Ospedaliero-Universitaria Pediatrica Meyer a valenza regionale. Le 3 Aziende Usl (Figura 1) sono: la Toscana Centro, la Toscana Nord-Ovest e la Toscana Sud-Est.

Ognuna di queste Aziende, anche riprendendo le esperienze precedenti, ha disegnato un proprio modello per definire il percorso clinico-assistenziale del paziente con scompenso cardiaco con lo scopo di renderlo omogeneo tra le varie strutture, di ottimizzare la qualità dell'assistenza ospedaliera e territoriale e di contenere il numero dei ricoveri ospedalieri.

Da sottolineare il rilevante ruolo che i medici internisti svolgono in ognuno dei 3 modelli.

Andremo a descrivere i modelli di ognuna delle 3 Aziende Usl, i quali, complice la recente pandemia da SARS-CoV-2, non sono ancora completamente implementati in tutto il territorio.

Corrispondente: Alberto Fortini, Medicina Interna Ospedale San Giovanni di Dio, Via di Torregalli 3, 50143 Firenze, Italia.

E-mail: alberto.fortini@uslcentro.toscana.it

Articolo pubblicato secondo la Creative Commons Attribution NonCommercial 4.0 License (CC BY-NC 4.0).

${ }^{\circ}$ Copyright: the Author(s), 2021

Licensee PAGEPress, Italy

QUADERNI - Italian Journal of Medicine 2021; 9(2):e14

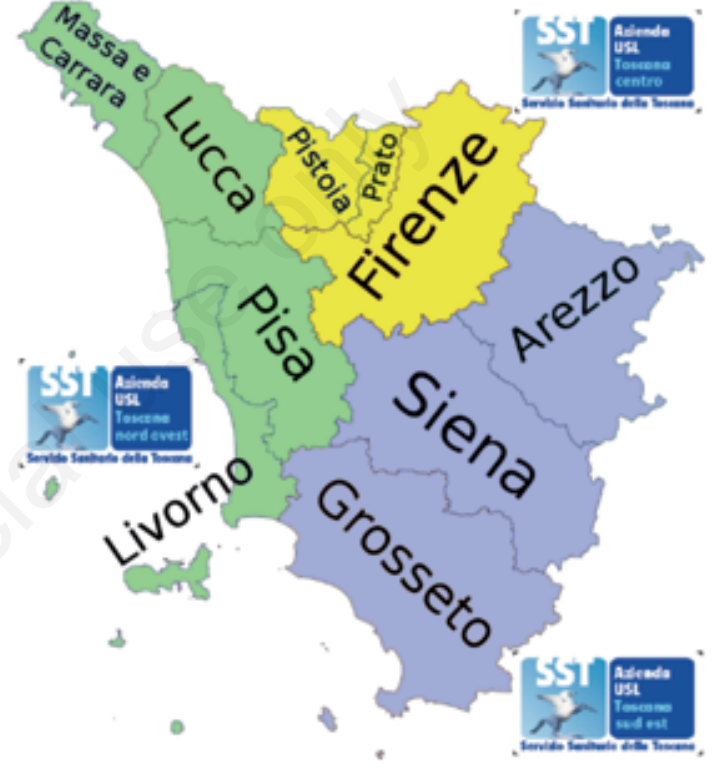

Figura 1. Localizzazione geografica delle 3 AA.VV. della Regione Toscana, nell'ambito delle quali operano le 3 Aziende Usl.

\section{USL Toscana Centro}

Nell'Azienda Toscana Centro all'inizio del 2016 è stato costituito un gruppo di lavoro multidisciplinare al quale ha aderito anche l'Azienda Ospedaliero-Universitaria di Careggi per definire il percorso gestionale del paziente con scompenso cardiaco. Il gruppo, nel quale vi era una rilevante componente di medici internisti, ha prodotto un documento in cui venivano affrontati: i) la gestione territoriale; ii) il ricovero ospedaliero; iii) la dimissione ed il follow-up.

\section{Gestione territoriale, caratteristiche principali}

Controlli ambulatoriali con monitoraggio dei parametri clinici ad opera della Medicina d'iniziativa (medico di medicina generale -MMG- ed infermieri 
territoriali); possibilità di accedere a posti CUP riservati per visita cardiologica territoriale, ECG, Holter ed ecocardiogramma; agevole accessibilità per il MMG all'ambulatorio ospedaliero dello scompenso cardiaco tramite numero telefonico dedicato; fasttrack per eseguire una consulenza cardiologica ospedaliera urgente entro 24-72 ore con numero verde CUP dedicato con accesso riservato al MMG.

\section{Ricovero ospedaliero}

Accesso al DEA, con definizione del trattamento ottimale e dei criteri di invio nel setting appropriato (dimissione, osservazione breve intensiva, degenza medica, cardiologica o geriatrica, terapia sub-intensiva cardiologica o rianimatoria). Degenza ospedaliera, con definizione degli accertamenti diagnostici iniziali, dei criteri per la valutazione ed il monitoraggio del compenso emodinamico, individuazione dei Centri di riferimento per le situazione più complesse (impianto di TAVI o mitral clip, scompenso refrattario, trapianto cardiaco).

\section{Dimissione e follow-up ospedaliero e territoriale}

Definizione dei criteri di dimissibilità e identificazione di uno score di stratificazione prognostica (Figura 2); in base al punteggio sono identificati 2 gruppi (basso ed alto rischio) con differenti percorsi di follow-up. I pazienti a basso rischio eseguiranno una visita presso l'ambulatorio ospedaliero dello scompenso entro 20-30 giorni e successivamente, se non divenuti instabili, verranno seguiti dal MMG e dall'infermiere territoriale a cadenza semestrale. Per quelli ad alto rischio è invece previsto un precoce controllo ambulatoriale e l'attivazione del monitoraggio infermieristico domiciliare con la possibilità di terapia infusiva di diuretici sempre domiciliare. Presupposto a questo programma post-dimissione è la creazione di un'organizzazione dedicata comprendente i MMG e gli infermieri territoriali, con uno stretto rapporto con l'ambulatorio dello scompenso ospedaliero; tale organizzazione è già attiva in alcune zone mentre in altre è in fase di realizzazione. Ultimo aspetto preso in con-

\section{Pdta dello Scompenso cardiaco della Azienda Usl Toscana centro: Tav Centro Hf Score}

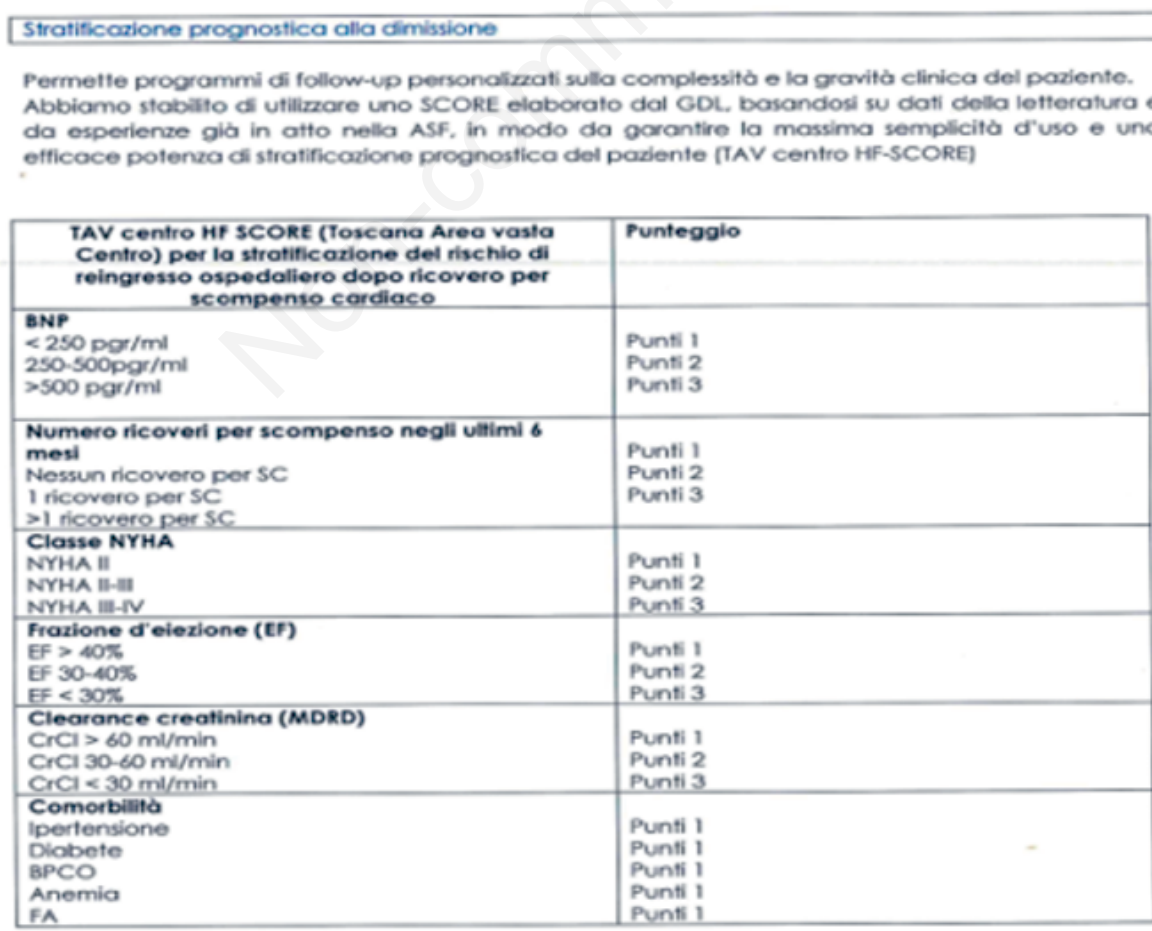

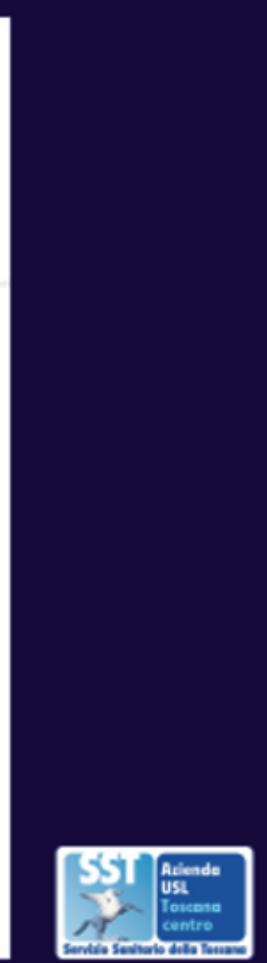

Figura 2. Score di stratificazione prognostica alla dimissione dei pazienti con scompenso. Score $\leq 15$ : rischio di reingresso basso; Score $>15$ : rischio di reingresso elevato. 
siderazione è la possibilità di cure palliative nei soggetti più gravi e/o con riserve funzionali esaurite.

Per valutare l'esito degli interventi messi in atto, sono stati identificati alcuni indicatori che devono essere periodicamente misurati, tra i quali: tasso di utilizzo della check list di dimissibilità e dello score prognostico; istituzione dell'infermiere case-manager di percorso e del monitoraggio clinico domiciliare con il MMG; istituzione collaborazione con i palliativisti; tasso di riammissione ospedaliera entro 30 giorni e a 3-6 mesi; tasso di mortalità annua per scompenso cardiaco.

Il progetto è stato attuato in maniera non uniforme nelle varie aree territoriali, ma comunque i primi risultati disponibili risultano incoraggianti per quanto riguarda il tasso di reammissioni ospedaliere che risulta ridotto in maniera significativa.

\section{USL Toscana Sud-Est}

Per affrontare la gestione delle patologie croniche, tra le quali lo scompenso cardiaco, l'Azienda Usl Toscana Sud-Est è partita dal modello di Sanità d'Inizia- tiva introdotto con il Piano Sanitario Regionale del 2008-2010. Inizialmente il modello prescelto è stato quello del Chronic Care Model, alla cui base vi è l'interazione tra il paziente, reso esperto da opportuni interventi di formazione e di addestramento, ed un Team multiprofessionale, composto da operatori socio-sanitari, infermieri e MMG. Tale modello ha mostrato, però, nel tempo, alcune criticità che ne hanno ridotto l'efficacia. Tra queste si sottolineano: i) la mancanza di una personalizzazione del percorso di cura; ii) l'autoreferenzialità degli operatori del Team, con conseguente frammentazione delle cure; iii) la mancanza di un rapporto strutturato tra Ospedale e Territorio.

L'evoluzione di tale modello è stata l'istituzione delle cosiddette Reti Cliniche Integrate e Strutturate, il cui asse portante è rappresentato dalla collaborazione tra le Unità operative (Uo) professionali ospedaliere, dove le Uo di Medicina Interna, vista la loro presenza capillare su tutti i 13 Ospedali dell'Azienda, svolgono un ruolo di grande rilevanza, le Aggregazioni funzionali territoriali (Aft) dei MMG. e i Team multiprofessionali delle Zone-Distretto/Società della Salute (Figura 3).

\section{Strumenti di integrazione Ospedale- Territorio nelle Reti cliniche}

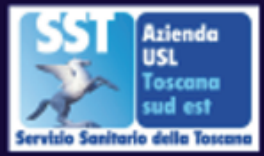

\section{INTEGRAZIONE OSPEDALE - TERRITORIO (apice della piramide)}

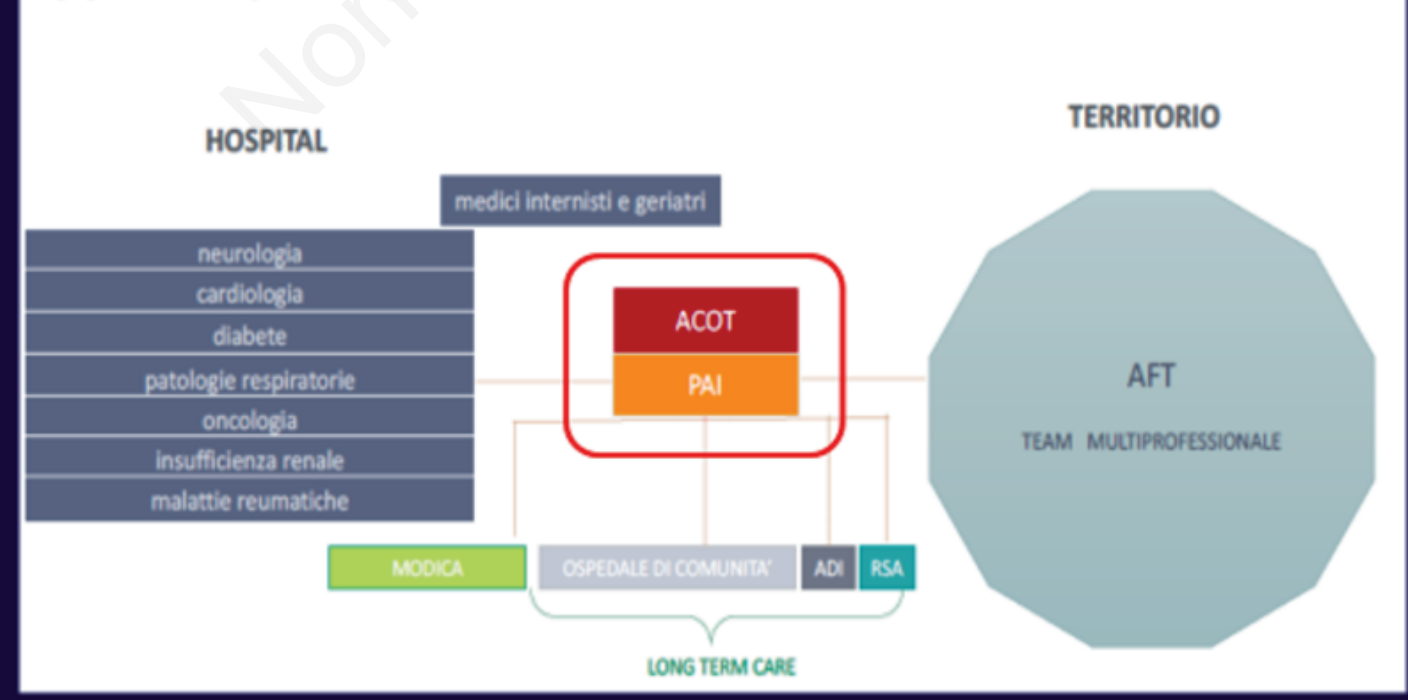

Figura 3. Reti cliniche integrate e strutturate (Azienda Usl Toscana Sud-Est). 
In particolare il collegamento tra Ospedale e Territorio è garantito dal rapporto tra i medici delle Uo cliniche che svolgono azione di referente nei confronti delle Aft e dei presidi territorali più in generale.

Fondamentale, infine, in questa organizzazione a Rete, l'attività dell'Agenzia per la continuità tra Ospedale e Territorio (Acot), struttura che coordina e gestisce i percorsi dei pazienti cronici dalla dimissione ospedaliera al rientro sul territorio (domicilio, Cure Intermedie, RSA), attraverso la definizione e l'applicazione dei Piani di assistenza individuale (Pai).

Per quanto riguarda lo scompenso cardiaco l'Azienda Usl Toscana sud-est ha, nel novembre 2017, deliberato uno specifico Piano diagnostico, terapeutico e assistenziale (PDTA) che prevede i seguenti step:

- identificazione ed arruolamento del paziente (avviene ad opera del MMG che individua, tra i suoi pazienti, quelli da avviare all'Ambulatorio pro-attivo);

- inizializzazione della fase di follow up (il medico, coadiuvato dall'infermiere, inquadra il paziente, richiede eventuali consulenze specialistiche e provvede alla stesura del Pai);

- ciclo di follow up (periodicità delle visite di controllo e degli esami, compreso l' ecocardiogramma, basata sulla classe funzionale NYHA);

- gestione del paziente instabile (a seconda del grado di instabilità clinica vengono attivati: i) il servizio di emergenza; ii) il ricovero ospedaliero; iii) la consulenza specialistica);

- monitoraggio del processo (si realizza attraverso la di un questionario da parte dell'infermiere al paziente, successivamente valutato in termini di raggiungimento degli obiettivi, da parte di un medico di Comunità dell'Azienda).

Le Uo ospedaliere referenti per lo scompenso cardiaco interagiscono con le strutture territoriali a seconda delle modalità con cui si sono organizzate.

Nell'esperienza della Uo Medicina Interna dell'Ospedale di Massa Marittima (Gr) si prevede la seguente organizzazione:

- presenza di due medici referenti per lo scompenso cardiaco, uno per ognuna delle due Aft che insistono sull'area territoriale di competenza (area delle Colline Metallifere).

- presenza di un Ambulatorio dedicato, con personale infermieristico, punto di riferimento per l'attività consulenziale prevista dal PDTA. Nell'Ambulatorio si svolge: i) attività diagnostica di $2^{\circ}$ livello; ii) attività prescrittiva, anche in termini di piani terapeutici; iii) attività di teleconsulto e di televisita; iv) attività educazionale rivolta al paziente $\mathrm{e} / \mathrm{o}$ al care giver; $\mathrm{v}$ ) attività di dimissione verso la propria residenza, istituzionalizzata o meno, o verso l'Ambulatorio protetto o verso i setting di degenza.
- presenza di un Ambulatorio protetto diurno. È un setting clinico, multidisciplinare e multiprofessionale, dedicato anche alla gestione di pazienti cronici, compresi quelli con scompenso cardiaco, che vanno incontro a riacutizzazioni, divenendo clinicamente instabili. Lo scopo di questa struttura è quello di gestire questi pazienti, evitando loro un inutile ricovero. Nell'Ambulatorio, provvisto di posti letto, è presente un equipe infermieristica dedicata, mentre i medici provengono dalle varie Uo che ivi operano. Si possono praticare terapie infusive e somministrare farmaci ospedalieri.

- Possibilità di ricovero ospedaliero in Area Medica (livello 2 di intensità di cura), all'interno della quale è presente un modulo High Care (livello $2 \mathrm{~A}$ di intensità di cura), dove i pazienti sono seguiti con un metodo di monitoraggio leggero $\mathrm{H} 24$.

\section{USL Toscana Nord-Ovest}

Fino a poco tempo fa i percorsi gestionali dello scompenso cardiaco nella Usl Toscana Nord Ovest sono stati estremamente difformi; in assenza di un intervento regolatorio generale ogni realtà locale si era mossa in maniera autonoma, in genere guidata da iniziative di singoli reparti o addirittura di singoli medici. Unico tratto comune risultava la centralità ospedaliera, nel senso che l'ambulatorio scompenso, nelle sue diverse declinazioni pratiche, era costantemente allocato in uno dei 13 ospedali dell'USL Toscana Nord Ovest. Altro elemento di rilievo era che il maggior numero di questi ambulatori era a conduzione esclusivamente cardiologica. La Medicina Interna giocava un ruolo numericamente secondario, con rare iniziative autonome e un caso di collaborazione operativa con la Cardiologia. Recentemente però la $\mathrm{Di}$ rezione Aziendale ha sentito l'esigenza di strutturare in maniera più organica questo settore assistenziale e ha dato mandato ad un referente Cardiologo, di organizzare un PDTA per lo scompenso cardiaco cronico strutturato come rete ospedale-territorio. La logica è stata quella di una presa in carico assistenziale del paziente delineando una organizzazione sempre in grado di dare una risposta alle diverse e necessità mutevoli che una malattia cronica ma evolutiva e con fasi di riacutizzazione/peggioramento presenta nel tempo. Gli obbiettivi di questo percorso sono stati esplicitati:

- disegnare una organizzazione in grado di prendere in carico il paziente già noto, prevenendo i ricoveri non programmati, riducendo la morbilità e la mortalità del paziente scompensato, identificando i pazienti a rischio di sviluppare lo scompenso;

- offrire un percorso di presa in carico di prossimità;

- garantire un percorso senza interruzioni al paziente, deframmentando l'accesso a cure e indagini;

- garantire le migliori cure per ogni stadio della malattia compreso il fine vita; 
- assicurare un'offerta assistenziale omogenea; identificare i pazienti che per necessità specifiche devono avere accesso a cure personalizzate sia per intensità di trattamento che per qualità di intervento;

- contribuire ad identificare le indagini e le cure più efficaci sul campo, la loro frequenza e la loro distribuzione territoriale rappresentando così anche un laboratorio di ricerca innovativa.

Sono quindi state fatte due riunioni preparatorie alle quali hanno partecipato i responsabili dei reparti di Cardiologia e Medicina e i responsabili della Medicina di Base e dei Distretti Territoriali dell'USL. Il percorso è stato disegnato con una logica di Rete che sostanzialmente assorbe e sviluppa il seguente concetto: ogni Zona, Ospedale, AFT, ed ogni organizzazione interna, professione o elemento del Terzo Settore costituisce un elemento della Rete che deve essere coordinato e governato nell'ottica di offrire una continuità assistenziale rispettando l'appropriatezza e la qualità delle cure ma anche la realtà geografica, privilegiando laddove possibile la prossimità del servizio erogato. Incrociando la complessità delle procedure necessarie con la complessità clinica del paziente si è cercato di organizzare il percorso in maniera che le condizioni più gravi o complesse possano convergere in pochi centri, magari anche distanti dalla residenza del paziente e all'opposto condizioni a minor complessità clinica e con necessità di procedure meno sofisticate possano trovare adeguata assistenza in aree di prossimità.

Nella costruzione di questo percorso a rete integrata sono stati individuati i seguenti attori cercando di attribuire a ciascuno compiti e responsabilità, sempre nell'ottica della reciproca integrazione: il paziente, il medico di medicina generale, l'infermiere di famiglia e di comunità, le cure domiciliari, l'agenzia di continuità ospedale-territorio (ACOT), l'hospice e le cure palliative, il terzo settore, l'ambulatorio scom- penso cardiaco, a conduzione prevalentemente cardiologica e unica struttura, in questo modello organizzativo, intermedia tra Ospedale e Territorio, lo specialista ambulatoriale convenzionato, il pronto soccorso-DEA, i reparti di degenza (cardiologie, medicine interne), il day hospital, il day service, le aree terapeutiche.

Le varie attività sono state schematicamente suddivise in tre fasi di percorso: i) accesso del paziente con scompenso in PS-DEA; ii) paziente con scompenso ricoverato in reparto medico; iii) paziente con scompenso gestito sul territorio. Ciascuna fase è poi stata articolata in più snodi di rete tra loro ingranati.

Ad oggi questo modello è in fase di implementazione; molte sono ancora le cose da fare e probabilmente altrettante quelle da modificare. Quello che però dovrà assolutamente rimanere immodificato è il principio fondante del sistema a rete integrata Ospedale-Territorio, unico modello gestionale che appare in grado di dare risposte convincenti alle necessità dei pazienti ma anche a quelle dell'organizzazione sanitaria.

\section{Considerazioni conclusive}

Seppure con modelli diversi, dovuti anche alla differenza geografica dei territori, in tutte e 3 le Aziende USL toscane sono stati definiti percorsi strutturati per la gestione del paziente con scompenso cardiaco che permettessero cure adeguate sia in ospedale che nel territorio, limitando solo ai casi più gravi il ricorso al ricovero ospedaliero. Tali programmi sono in via di attuazione con attuale disomogeneità fra le varie zone ed il processo ha subito un rallentamento per la pandemia virale in corso. I primi risultati disponibili sono comunque incoraggianti ed il proposito è di implementare il percorso in maniera diffusa nei prossimi 12 anni. 


\title{
Modelli a confronto nei percorsi gestionali dello scompenso cardiaco: l'esperienza marchigiana
}

\author{
Nicola Tarquinio \\ Unità Operativa Complessa di Medicina Interna, INRCA-IRCSS di Osimo (AN), Italia
}

L'ambulatorio ha sede nel Presidio ospedaliero di Osimo, e sfrutta i nuovi spazi messi a disposizione dalla Direzione Sanitaria con i lavori portati a termine nell'ex reparto di Pediatria ( $2^{\circ}$ piano) nel 2018. Gli stessi spazi ospitano anche l'ambulatorio di cardiologia ed il laboratorio di ecocardiografia/ecografia vascolare: tali servizi afferiscono tutti all'U.O.C. di Medicina Interna dello stesso nosocomio (Figura 1). L'ambulatorio è attivo dal 2015, viene gestito dall'internista con competenze in campo cardiovascolare, e dal 2019 è stato anche riconosciuto e reso istituzionale dall'INRCA: sono stati predisposti a CUP 5 posti a settimana con prenotazione mediante la modalità $a c$ cesso diretto direttamente dal medico accettante e gestore, eventualmente anche ampliabili in base alle necessità del momento in quella stessa seduta, che possono essere utilizzabili sia per le prese in carico che per le visite di controllo (con eventuale titolazione

Corrispondente: Nicola Tarquinio, Unità Operativa Complessa di Medicina Interna, INRCA-IRCSS, Presidio Ospedaliero di Osimo, INRCA IRCCS, Via Giacomo Leopardi 15, 60027 Osimo (AN), Italia.

E-mail: n.tarquinio@inrca.it

Articolo pubblicato secondo la Creative Commons Attribution NonCommercial 4.0 License (CC BY-NC 4.0).

${ }^{\circ}$ Copyright: the Author(s), 2021

Licensee PAGEPress, Italy

QUADERNI - Italian Journal of Medicine 2021; 9(2):e15 dei farmaci e follow-up ecografico) per pazienti ambulatoriali, o che abbiano avuto accesso al Pronto Soccorso e poi dimessi, o che abbiano superato il $30^{\circ}$ giorno dalla dimissione ospedaliera (Tabella 1). Tutte le prestazioni accessorie connesse alla visita (ecocardiogramma; ecografia polmonare; ECG; emogasanalisi; spirometria; prelievi ematici, ecc.) sono regolarizzate mediante impegnativa dematerializzata redatta dallo stesso medico ed agganciata alla stessa prestazione principale erogata a favore del paziente (prima visita per presa in carico - ambulatorio scompenso cardiaco, o visita di controllo - ambulatorio scompenso cardiaco), anch'essa coperta da impegnativa dematerializzata e redatta da esso.

In aggiunta a questi 5 posti a CUP, nella stessa seduta sono previsti ulteriori accessi per i controlli di follow-up a breve termine post-dimissione (dall'U.O. Medicina Interna o altri reparti), in numero flessibile in base alle esigenze contingenti ed alla fattibilità. Tutte le visite programmate presso l'ambulatorio confluiscono in un'agenda interna elettronica conservata sotto forma di file Excel presso il server dell'Azienda, visibile solamente ai medici dell'U.O.C di Medicina Interna ed agli infermieri dedicati alla raccolta delle prenotazioni. Ciò al fine di comporre una seduta dove non possano verificarsi sovrapposizioni di orari e garantire adeguato distanziamento degli orari stessi tra un paziente e l'altro in tutte le sedute.

I Medici di Medicina Generale possono prendere direttamente contatto con il medico gestore dell'ambulatorio per la proposta di valutazione del proprio as-

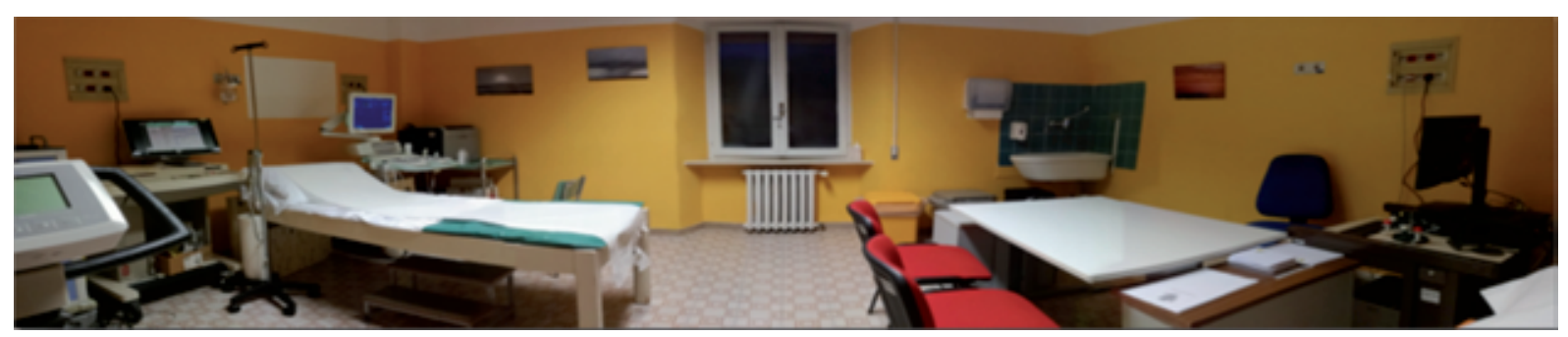

Figura 1. L'ambulatorio di cardiologia dell'U.O.C. di Medicina Interna di Osimo - INRCA. 
sistito e/o presa in carico, dietro adeguata presentazione del caso e concordandone preventivamente il percorso (Figure 2 e 3 ).

Durante la seduta tutti i pazienti vengono inseriti nel sistema informatico aziendale SIS (pertanto vi è traccia di ogni prestazione erogata per ciascun paziente), così come le prestazioni aggiuntive che possono essere decise dal medico anche al momento della visita.

Presso il nostro ambulatorio sono stati valutati e gestiti sino ad ora circa 300 pazienti.

Tabella 1. Organizzazione Ambulatorio Scompenso Cardiaco a gestione internistica dell'U.O.C. Medicina Interna di Osimo - INRCA IRCCS.

Presenza di un'infermiera professionale dedicata in stanza adiacente (prelievi eseguibili al momento della visita se ritenuti indicati dal medico, raccolta anamnesi/assistenza infermieristica, raccolta parametri vitali, esecuzione ECG, posizionamento monitoraggio pressorio 24 ore/Holter-ECG) presso l'ambulatorio di Cardiologia del Presidio di Osimo

Un'ampia stanza per ECG, lettino + ecografo Esaote Mylab60 dotato di sonde cardiologica, convex e lineare (con stampante a colori in formato A4), per esecuzione in autonomia da parte del medico gestore dell'ambulatorio di ecocardiogramma, ecografia del torace, CUS, ecoscopia addominale, test da sforzo al cicloergometro

Un PC collegato in rete intranet/internet per consultazione referti, esami di laboratorio eseguiti anche da esterni nei laboratori pubblici del1'Asur Marche Area Vasta 2, cartella clinica elettronica per ogni paziente, aggiornabile ad ogni controllo sul sistema SIS dell'INRCA

Possibilità di eseguire sul momento esami di laboratorio, Rx torace, spirometria con DLCO, emogasanalisi, six minute walking test, test da sforzo al cicloergometro, posizionamento di monitoraggio automatico pressorio delle 24/48 ore o Holter-ECG (con lettura e refertazione in autonomia) titolazione farmaci con osservazione clinica e monitoraggio dei parametri, infusione diuretici ad alto dosaggio. In quest'ultimo caso vi è un collegamento diretto con il Day Hospital dell'U.O. (che è in fase di potenziamento) per la trasformazione della prestazione ambulatoriale in ricovero in regime di Day Hospital, qualora il paziente venga ritenuto gestibile in tal senso senza la necessità del ricovero ospedaliero. La stessa soluzione viene adottata qualora il paziente sia anche portatore di una fibrillazione atriale persistente ed abbia indicazione all'esecuzione di una cardioversione elettrica in elezione, in precedenza programmata (anche in questo caso l'U.O. è autonoma nella sua gestione e nella procedura)

Possibilità di eseguire consulenze pneumologica, ematologica, reumatologica e diabetologica se ritenute utili, con eventuale prescrizione di farmaci specialistici specifici e relative prestazioni

Percorso, anche fast track, di presa in carico del paziente scompensato entro 7-10 giorni dall' evento acuto/dimissione, con follow-up più intensivo riservato ai pazienti ad elevato rischio di instabilizzazione, eventualmente con: titolazione della terapia farmacologica; rivalutazione ecocardiografica; gestione dei sintomi e delle eventuali terapie aggiuntive, con accertamenti ulteriori; ricognizione e razionalizzazione terapia farmacologica al fine di favorire anche l'aderenza del paziente, management delle comorbilità associate; link con Emodinamica, U.O.C. Cardiologia INRCA, U.O. Cardiochirurgia degli Ospedali Riuniti di Ancona, eventuale indicazione alla riabilitazione cardiologica 


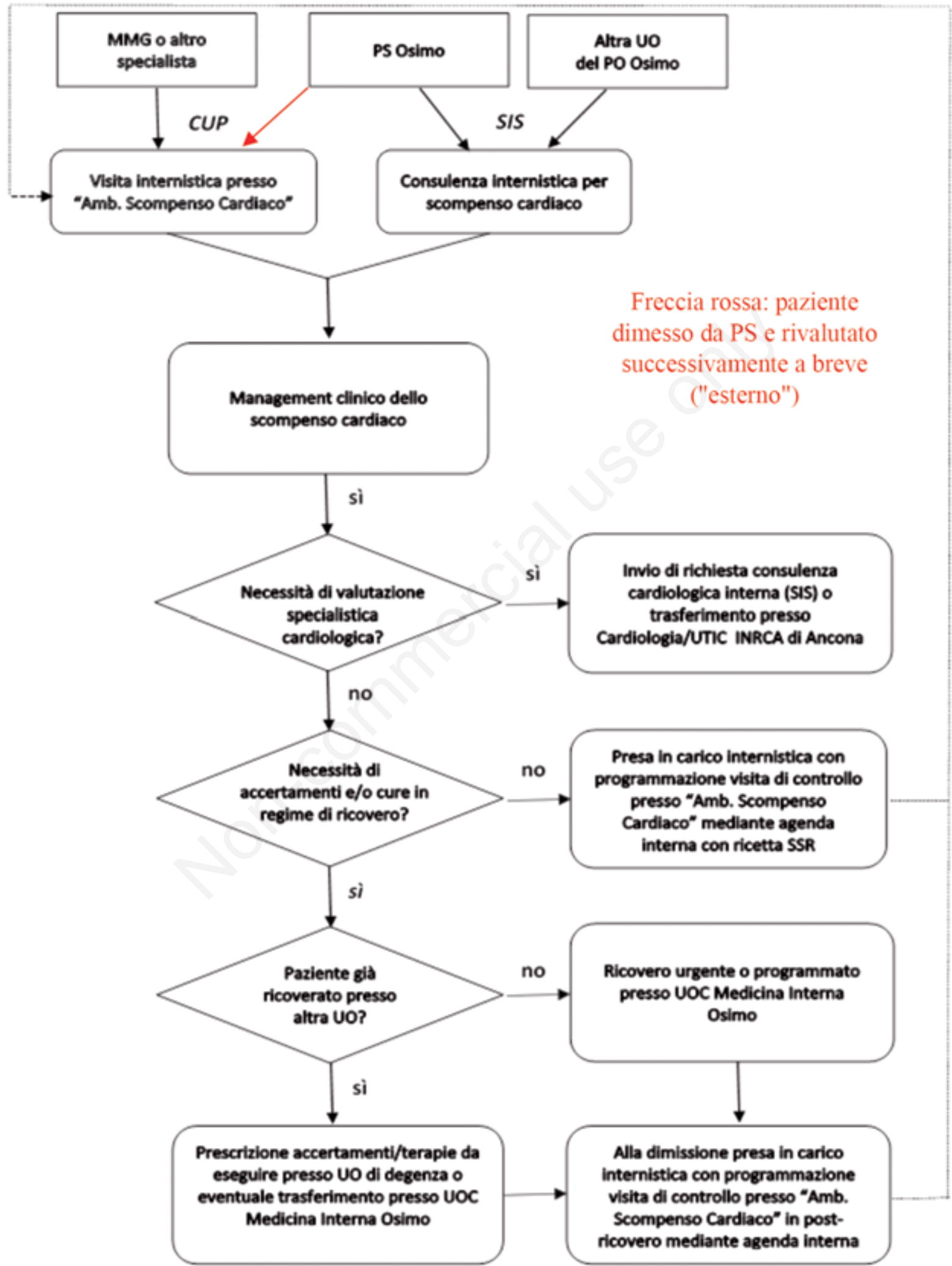

Figura 2. Flow chart per presa in carico specialistica internistica del paziente con scompenso cardiaco. 
Medico di Medicina Generale

Centro antidiabetico Osimo
Ambulatorio territoriale

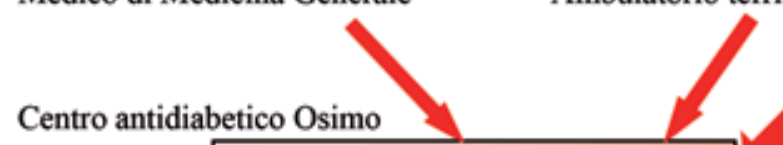
ambulatori di Cardiologia di Osimo e dell'INRCA di Ancona

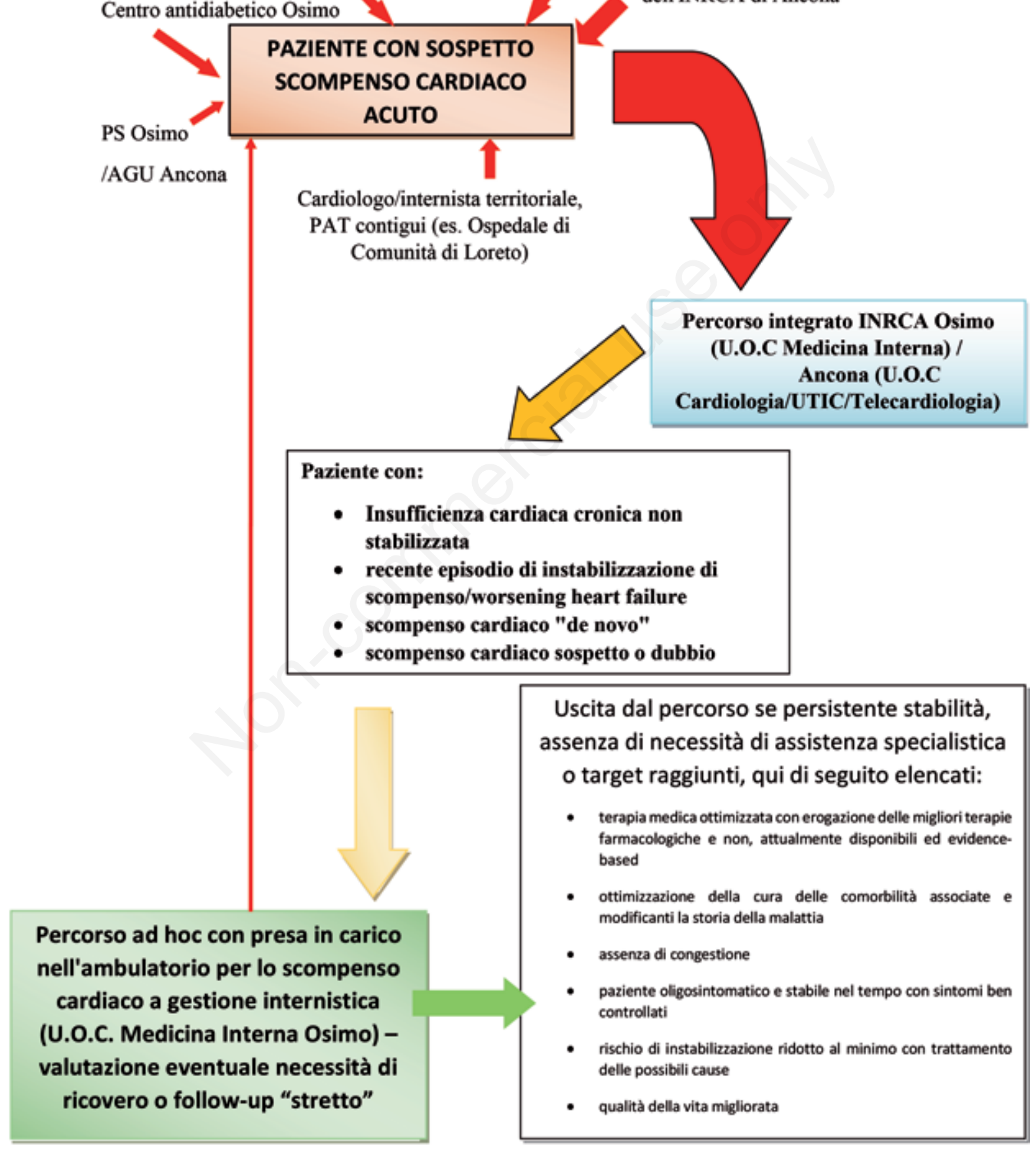

Figura 3. Percorso proposto del paziente con insufficienza cardiaca cronica o worsening heart failure, adattato alla realtà ospedaliera dei presidi INRCA di Osimo ed Ancona. 


\section{Modelli a confronto nei percorsi gestionali dello scompenso cardiaco: l'esperienza savonese. Il progetto PONTE}

Paola Gnerre, ${ }^{1}$ Monica Pivari, ${ }^{1}$ Eugenia Monaco, ${ }^{1}$ Maria Cristina Pistone, ${ }^{1}$ Massimiliano Basso, ${ }^{2}$ Pietro Bellone,${ }^{2}$ Antonella Visconti, ${ }_{1}^{1}$ Barbara Sardo, ${ }^{1}$ Barbara Zanella, ${ }^{1}$ Anna Maria Saccone, ${ }^{3}$ Antonietta Piras, ${ }^{3}$ Anna Santo, ${ }^{1}$ Fabiana Bernardi, ${ }^{1}$ Silvana Lapponia, ${ }^{1}$ Elena Montanari, ${ }^{1}$ Roberta Rapetti, ${ }^{4}$ Rodolfo Tassara, ${ }^{5}$ Lionello Parodi ${ }^{5}$

${ }^{1}$ Medicina 2 e Cure Intermedie, Ospedale San Paolo, Savona; ${ }^{2}$ Cardiologia, Ospedale San Paolo, Savona; ${ }^{3}$ Area infermieristica Ospedale e Territorio Ospedale San Paolo, Savona; ${ }^{4}$ Coordinamento delle professioni sanitarie, Ospedale San Paolo, Savona; ${ }^{5}$ Medicina 1 e Ematologia, Ospedale San Paolo, Savona, Italia

\section{Introduzione}

Tutti i Servizi Sanitari dei paesi devono affrontare tre grandi sfide: le crescenti esigenze cliniche a fronte di servizi ridotti per la medicina acuta, il progressivo invecchiamento della popolazione che determina un incremento della complessità e dei bisogni assistenziali dei pazienti e la scarsa continuità e frammentazione delle cure nel passaggio ospedale-territorio. ${ }^{1} \mathrm{Il}$ cosiddetto paradosso della medicina moderna, è che continuiamo a praticare una medicina per acuti in un mondo di malattie croniche, dove l'assistenza deve essere proattiva (intesa come anticipazione dei bisogni), continua e fondata sulla gestione globale del paziente. Una disanima delle evidenti disfunzioni correlate alla gestione delle malattie croniche ha dimostrato come troppi medici sono coinvolti nella gestione dello stesso paziente con possibilità di consigli contrastanti, come aumenti il rischio di sottovalutare sintomi/segni di peggioramento di una malattia cronica, e come si verifichi una scarsa educazione del paziente, famigliari e caregivers con scarsa aderenza al trattamento e incrementato rischio di riacutizzazioni della malattia. ${ }^{2}$ L'attuale incapacità ad affrontare la complessità di pazienti sempre più anziani e con numerose comorbidità e la mancanza di una vera continuità tra i vari setting assistenziali, soprattutto tra ospedale e territorio, si traduce pertanto in un utilizzo inappropriato delle risorse

Corrispondente: Paola Gnerre, Medicina 2, Ospedale San Paolo, via Genova 30, Savona, Italia.

E-mail: pgnerre@yahoo.it

Articolo pubblicato secondo la Creative Commons Attribution NonCommercial 4.0 License (CC BY-NC 4.0).

${ }^{\circ}$ Copyright: the Author(s), 2021

Licensee PAGEPress, Italy

QUADERNI - Italian Journal of Medicine 2021; 9(2):e16 e in un aumentato rischio per il paziente con incremento del ben noto fenomeno del revolving doors. La vera sfida è pertanto una reinvenzione radicale dell'organizzazione ospedaliera attraverso l'implementazione di nuovi modelli organizzativi di integrazione tra ospedale e territorio che consentano la riduzione dei i ricoveri ospedalieri ma nel contempo garantiscono al paziente di ricevere cure sicure, di alta qualità e incentrate sui loro bisogni in un ambiente adeguato. ${ }^{3}$ Tutto ciò dovrebbe pertanto contribuire al delineamento di programmi di disease management attraverso l'implementazione di strutture intermedie, i Day service multidisciplinari e le Aree a gestione infermieristiche, che funzionino da ponte tra ospedale e territorio. La nostra Regione e in particolare la nostra ASL che presenta un tasso di vecchiaia più elevato rispetto alle altre ASL Liguri (ASL 1, ASL 3, ASL 4, ASL 5) e quindi con un maggior numero di pazienti cronici complessi si presta bene all'implementazione di questi programmi di disease management. In Figura 1 è mostrato il rapporto tra numeri residenti e over 65 che rappresentano circa $1 / 3$ del totale dei residenti dei 4 distretti sanitari afferenti alla nostra ASL (Fonte: sito ASL 2 Sistema Sanitario Ligure ${ }^{4}$ ).

\section{Il progetto PONTE}

Da Novembre 2013 presso il presidio Ospedaliero San Paolo di Savona afferente all'ASL 2 Sistema Sanitario Ligure è attivo il P.D.T.A sullo Scompenso Cardiaco (SC) attraverso l'implementazione del Progetto P.O.N.T.E (A Pilot study for implementation of regiOnal guidelines for the integrated maNagement between hospital and Territory of hEart failure ligurian population). Il progetto arruola tutti i pazienti ricoverati presso le medicine con diagnosi di ammissione di scompenso cardiaco e età $\geq 65$ anni. In Figura 2 è presentata la distribuzione dei ricoveri per scompenso cardiaco presso la nostra ASL. Obiettivo del progetto è di evitare le ri- 
ospedalizzazioni per scompenso cardiaco attraverso una presa in carico precoce del paziente (Tabella 1). Il progetto utilizza una modalità di disease management basata sul case manager infermieristico in congiunzione con il medico di area internistica e cardiologica. Il paziente scompensato che afferisce al progetto è generalmente un paziente in classe NYHA 3-4 che si caratterizza per la presenza di multiple comorbidità (broncopneumopatia cronica ostruttiva, insufficienza renale, diabete mellito, cardiopatia ischemica, ipertensione arteriosa, fibrillazione atriale, dislipidemia, arteriopatia periferica sono le più frequenti) con frequenti riacutizzazioni e fasi critiche di instabilità clinica indotte sia dalla malattia indice (lo scompenso cardiaco) che dalle comorbidità associate e che richiede frequentemente il ricovero. Il paziente affetto da scompenso cardiaco è infatti un esempio paradigmatico di paziente affetto da comorbidità in cui alla malattia indice dominante (scompenso cardiaco) si associano specifiche malattie correlate. ${ }^{5}$ La comorbidità è un problema importante e in continua crescita, soprattutto nelle società con invecchiamento della popolazione in grado di influenzare non solo la severità dei sintomi e la qualità della vita del paziente ma anche la prognosi correlata al rischio di morte e ospedalizzazione. ${ }^{6} \mathrm{Al}$ paziente oltre ad un regolare follow-up telefonico e clinico viene fornito, in caso di peggioramento del quadro clinico, la possibilità di accedere direttamente al Day Hospital senza recarsi in Pronto Soccorso per essere sottoposto alle cure del caso che generalmente prevedono la cura della malattia indice ma in alcuni momenti anche l'eventuale trattamento delle comorbidità associate. Inoltre al paziente viene garantito attraverso la figura del case manager infermieristico un processo di educazione terapeutica (Figure 3 e 4). L'educazione terapeutica è un nuovo paradigma di cura per le malattie croniche che migliora non solo le conoscenze dei pazienti, ma soprattutto le competenze, intese come saper fare, le capacità relazionali, intese come saper essere favorendo l'autonomia della scelta ed un senso di responsabilità del malato per renderlo protagonista attivo della cura aumentando l'aderenza alla terapia. ${ }^{7}$ Da Novembre 2019 nel nostro presidio Ospedaliero è stato inaugurata l'Area infermieristica Ospedale-Territorio come ulte-

Tabella 1. Obiettivo generale e obiettivi specifici del progetto PONTE.

Obiettivo generale

Obiettivi del GOI della cronicità è la creazione di un nuovo modello di cura che favorisca l'integrazione e il coordinamento tra Ospedale e Territorio per contribuire a migliorare la tutela delle persone affette da malattie croniche rendendo più efficaci ed efficienti i servizi sanitari in termini di prevenzione e assistenza assicurando maggior uniformità ed equità di accesso ai cittadini

Obiettivi specifici

La riduzione dei re-ricoveri dei pazienti affetti da malattie croniche

L'identificazione dell'internista del Day Hospital/Day Service come figura di riferimento del percorso del paziente nella fase di acuzie, come coordinatore dei vari specialisti e come perno di congiunzione con il medico di medicina generale per evitare la duplicazione di servizi e indagini e l'omissioni di prestazioni necessarie.

L'implementazione di un modello di cura proattivo con l'avvio di un programma educazionale del paziente, dei familiari e del caregivers attraverso la figura del case manager infermieristico con l'obiettivo di migliorare la conoscenza della malattia e in particolare dei sintomi/segni di peggioramento, di aumentare l'aderenza al trattamento e di ridurre il rischio di eventi avversi correlati al trattamento

La creazione di una cartella clinica centralizzata sul paziente fruibile da tutti gli operatori impegnati nel processo di cura del paziente

Formazione del personale coinvolto per l'acquisizione di un know-how necessario per coordinare e gestire i pazienti con complesse e multiple comorbidità

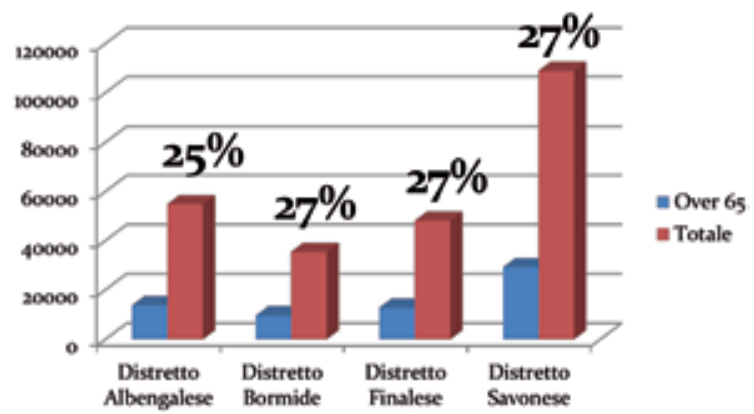

Figura 1. Rapporto over 65 su totale residenti dei 4 distretti sanitari dell'ASL 2 Sistema Sanitario Ligure.

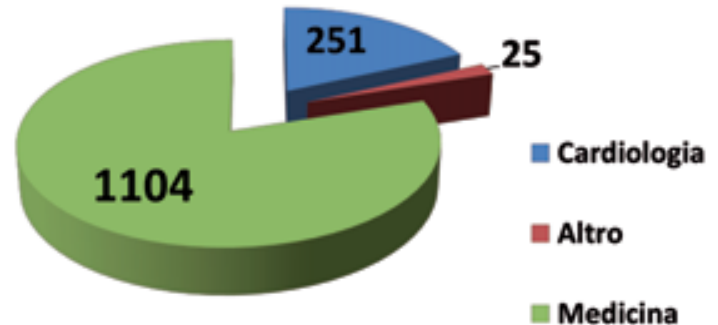

\section{Pazienti totali 1380}

Figura 2. Distribuzione per reparti di cura dei pazienti ricoverati per scompenso cardiaco presso la nostra ASL anno 2016. 
Quando contattare l'infermiere o il medico:

Aumento di peso superiore a $3 \mathrm{~kg}$ che non ha risposto all'aumento della dose del diuretico.

$\checkmark$ Incertezza relativa alla posologia del diuretico da incrementare.

$\checkmark$ Comparsa di edemi degli arti o gonfiore dell'addome.

$\checkmark \quad$ Peggioramento della dispnea per livelli di esereizio leggero.

- Comparsa di impossibilità a dormire supini nel ketto e di risvegli dal sonno per mancanza di fiato.

$\checkmark$ Tosse in peggioramento, tosse stimolata dalla posizione sdraiata.

Capogiri in peggioramento oppure di recente comparsa non collegati ad improvvisi cambiamenti di posizione.

$\checkmark$ Cardiopalmo prolungato.

Quando richiedere l'intervento del 112:

- Grave difficolta' respiratoria o sensazione di soffocamento

- Dolore o senso di oppressione al petto

- Svenimento o perdita di coscienza

- Improvviso disturbo della parola o della vista

- Battito cardiaco troppo veloce o troppo lento associato a sintomi

\section{Figura 3. Brochure esplicativa del progetto PONTE.}

Dipartimento di Medicina Interna Durctive Dote R. Tmane SC Medicina Interna

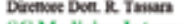

SC Medicina Interna ?

Dinthose Doet L. Preod

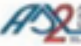

Savea

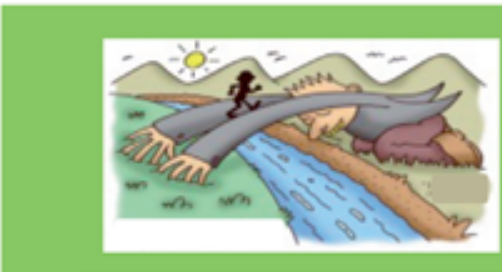

\section{Progetto P.O.N.T.E}

Studio pilora per I'implementazione di linee guida negionali per la gestione integrata tra aspedale e territorio dei pazienti afferti da scompenso

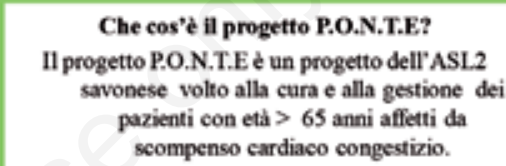

Che cos'è il progetto P.O.N.T.E?

Il progetto P.O.N.T.E è un progetto dell'ASL.2 savonese volto alla cura e alla gestione dei pazienti con età > 65 anni affetti da scompenso cardiaco congestizio.

Dipartimento di Medicina Interna

Medicina Interna 1 ed ematologia

Direttore Dr. Tassara Rodolfo

Medicina Interna 2 e Cure intermedie

Direttore Dr. Parodi Lionello

\section{ZONA VERDE}

Questa è la zona in cui Lei deve rimanere!

Non ha il "fiato corto" né mancanza o difficolta

a respirare

Non sono presenti edemi (gambe piedi pancia gonfi)

II peso è stabile

Non ci sono dolori al torace

\section{ZONA GIALLA}

Attenzione!

$\checkmark$ Aumento del peso $1 / 1,5 \mathrm{Kg}$ in poco tempo

$\checkmark$ Aumento della difficoltà a respirare specie per piccoli sforzi

$\checkmark$ Respiro difficoltoso durante la notte

(uso di cuscini o riposo obbligato in sedia)

$\checkmark$ Aumento della presenza di edemi (gambe piedi pancia gonfi)

$\checkmark$ Vertigini o svenimenti aumento della stanchezza e diminuzione delle energie

\section{ZONA ROSSA \\ Emergenza!}

$\checkmark$ Improvvisa e pesante difficoltà a respirare anche in assenza di sforzi fisici

$\checkmark$ Dolore al torace

Ricordatevi di:

$\checkmark$ Pesarvi ogni mattina prima di colazione e dopo avere urinato

$\checkmark$ Assumere le medicine prescritte ad orario

Figura 4. I semafori da ricordare!

$\checkmark$ Mantenere una dieta povera di sale

$\checkmark$ Alternare attività fisica e riposo 
riore perno di congiunzione tra ospedale e territorio. Il processo di educazione terapeutica di eventuali pazienti ricoverati viene pertanto anche garantito da questa area che accoglie eventuali pazienti che necessitano di un periodo di stabilizzazione clinica.

\section{Risultati}

La valutazione della prima diagnosi di scompenso cardiaco (codici SDO 428, 398.91, 402.01, 402.11, 02.91, 404.03, 404.93, 404.13, 404.91) e della prima diagnosi di insufficienza respiratoria acuta (codici 518.81) e seconda diagnosi di ammissione di scompenso cardiaco presso la nostra ASL (Figura 5) ha dimostrato una riduzione dei ricoveri per scompenso cardiaco del 13\% nel primo anno di attività (2013-2014) e del 15\% dopo 6 anni. Va comunque considerato che per gli anni 2013 e 2014 la valutazione dei ricoveri per scompenso cardiaco è stata fatta considerando solo la prima diagnosi di dimissione per scompenso cardiaco. Pertanto verosimilmente il numero di ricoveri per gli anni 2013 e 2014 è sottostimato. I nostri risultati sono in linea con i dati della letteratura internazionale che dimostrano come l'implementazione di programmi multidisciplinari post-dimissione per la presa in carico precoce e costante del paziente affetto da scompenso cardiaco cronico inducono una riduzione del 35\% delle riammissioni ospedaliere. ${ }^{8,9}$

\section{Conclusioni}

Il progresso scientifico ha sicuramente migliorato il processo di cura per i pazienti con una singola patologica medica ma la maggior parte dei pazienti che si recano in Ospedale sono affetti da multiple comorbidità spesso in aggiunta a quadri di deterioramento cognitivo che ne complicano ulteriormente la gestione. ${ }^{1}$ L'attuale approccio focalizzato sulla cura della singola malattia dovrebbe essere pertanto sostituito con una visione olistica incentrata sul paziente. Il cambiamento demografico della popolazione con il progressivo incremento dell'età media ha determinato la comparsa di un nuovo modello di paziente che a causa delle sue multimorbidità si caratterizza per una crescente complessità non solo dal punto di vista clinico ma anche terapeutico-gestionale. ${ }^{2}$ L'aumento della spettanza di vita correlati ad una crescente domanda di assistenza sanitaria sono però anche intimamente correlati all'incontrollato incremento della spesa sanitaria verificatosi negli ultimi decenni. Se consideriamo infatti che tali patologie assorbono più del $70 \%$ dei costi sanitari diretti, provocando altrettanti costi indiretti e sono in costante aumento a causa dell'invecchiamento gene-

\section{Numero ricoveri per Scompenso Cardiaco ASL 2.}

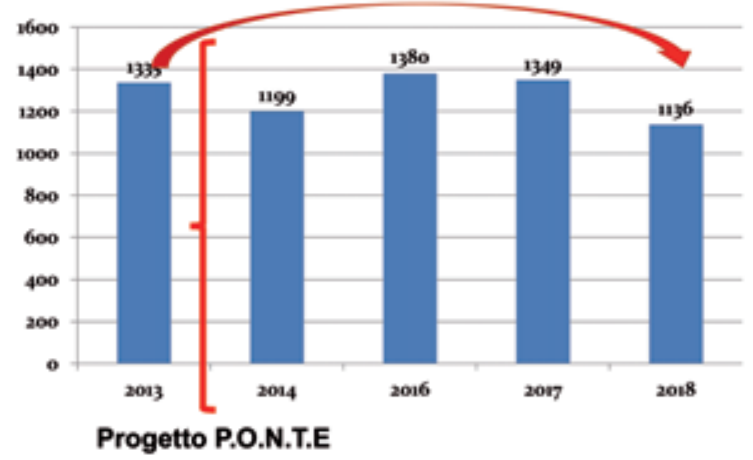

Figura 5. Distribuzione dei ricoveri per scompenso cardiaco dal 2013 al 2018 presso la nostra ASL.

rale della popolazione, possiamo affermare che le patologie croniche rappresentano l'emergenza del $3^{\circ}$ millennio. È quindi emersa la necessità da un lato di investire nella prevenzione, riducendo i fattori di rischio individuale, dall'altro di ricercare modelli di cura che agendo in maniera interdisciplinare e integrata inducano una presa in carico globale del paziente e prevengano le riacutizzazioni di malattie. ${ }^{8} \mathrm{Il}$ buon funzionamento dei sistemi sanitari e la loro tenuta si rapporta quindi con la capacità di fornire al paziente l'intervento più efficace per un bisogno specifico (appropriatezza clinica) e nel contempo di erogare la prestazione più efficace, più gradita, più sicura e più economica nel contesto assistenziale più idoneo all'erogazione delle cure (appropriatezza organizzativa). Sono considerati inappropriati e quindi indicatori di scarsa performance $\mathrm{i}$ casi trattati in regime di ricovero che le strutture sanitarie potrebbero trattare in un diverso setting assistenziale con identico beneficio per il paziente e con minor impiego di risorse. È andata così delineandosi la ricerca di percorsi standard i Percorsi Diagnostico Terapeutici e Assistenziali (PDTA) primo passo per definire dei percorsi di cura tra ospedale e territorio il più possibili uniformi, efficienti e standardizzati. ${ }^{10}$ Per un paziente giovane affetto da una singola patologia anche complessa, la definizione di un PDTA risulta relativamente semplice ma nel caso di un paziente fragile, pluripatologico ed anziano il tutto diventa più complesso. Un percorso efficiente deve offrire al malato, alla famiglia e al caregiver la possibilità di ricevere un'assistenza sicura e di qualità nel setting di cura più adeguato. Non esiste un modello organizzativo in sanità perfetto, ma ogni modello deve essere implementato nel contesto sociale, economico e culturale in cui si applica. Nella realtà sanitaria si percepisce quotidianamente un distacco tra territorio 
e ospedale con difficoltà concrete nel garantire ai pazienti un corretto percorso assistenziale, sia all'interno che all'esterno dell'Ospedale. La riorganizzazione sia degli ambiti Ospedalieri che Territoriali ad oggi non è riuscita a realizzare la continuità di cura elemento essenziale per una sanità efficiente e competitiva. Basterebbe un semplice ponte per permettere a due rive opposte di poter comunicare nell'ottica di una filosofia di cura patient centred care e di una filosofia organizzativa ad intensità di cura. Funzione del medico ospedaliero e del medico territoriale deve pertanto essere quella di integrarsi e di coordinarsi per poter gestire casi a varia complessità e intensità nel setting più idoneo che possa essere il Territorio, l'Ospedale o una struttura intermedia. Non più una modalità di cura per luoghi ma una modalità di cura per caso. Ogni struttura ospedaliera dovrebbe quindi implementare degli ambulatori della complessità come i Day Service multidisciplinari per la gestione e il monitoraggio di patologie croniche complesse che data la loro complessità non possono essere gestite a livello territoriale ma che attraverso una presa in carico precoce possono evitare il ricovero ospedaliero. Tali strutture devono diventare il perno di congiunzione tra Ospedale e Territorio.

\section{Bibliografia}

1. McPhail SM. Multimorbidity in chronic disease: impact on health care resources and costs. Risk Manage Healthcare Policy 2016;9:143-56.

2. Lehnert T, Heider D, Leicht H, et al. Review: health care utilization and costs of elderly persons with multiple chronic conditions. Med Care Res Rev 2011;68:387-420.

3. Barlow J, Wright C, Sheasby J, et al. Self management approaches for people with chronic conditions: a review. Patient Educ Couns 2002;48:177-87.

4. Sistema Sanitario Regione Ligura. Asl 2; https://www. asl2.liguria.it

5. Norton C. Epidemiology and cost of advanced heart failure. Progr Cardiovasc Dis 2011;54:78-85.

6. Tinker A. How to improve patient outcomes for chronic disease and comorbidities. Health Catalyst

7. Therapeutic Patient Education Continuing Education Programmers for Health Care Providers in the Field of Prevention of Chronic Diseases. World Health Organization; 1998. Available from: www.euro.who.int/ data/assets/ pdf_file/0007/.../E63674.pdf

8. Elrodt G. Evidenced-based disease management. JAMA 1997;278:1687-92.

9. Gonseth J. The effectiveness of disease management programs in reducing hospital re-admission in older patients with heart failure: a systematic review and meta-analysis of published reports. Eur Heart J 2004;25:1570-95.

10. Piano nazionale della cronicità; www.salute.gov.it 


\title{
Modelli a confronto nei percorsi gestionali dello scompenso cardiaco: l'esperienza campana
}

\author{
Fernando Gallucci \\ UOC Medicina Interna 3, AORN A. Cardarelli, Napoli, Italia
}

\section{Introduzione}

La popolazione campana è caratterizzata da una delle maggiori percentuali di soggetti ad alto rischio cardiovascolare in Italia e, quindi, anche da un'incidenza e prevalenza di cardiopatia ischemica, diabetica ed ipertensiva mediamente alte (Progetto PASSI, Atlante ANMCO delle malattie cardiovascolari). Dal momento che queste cardiopatie, a loro volta, rappresentano le principali cause dello scompenso cardiaco (SC), ne deriva un incremento significativo della incidenza e prevalenza anche di tale sindrome in Campania.

Un dato indispensabile per valutare l'adeguatezza delle risposte assistenziali e per fornire basi solide al processo di regionalizzazione della Sanità è rappresentato dalla conoscenza della realtà epidemiologica. ${ }^{1}$ Considerando nello specifico la Campania, il tasso di ricovero per SC risulta di 343,3/100.000 abitanti, rappresentando la prima causa di ricovero nei soggetti over 65 anni (1369/100.000 abitanti). ${ }^{2,3}$ Inoltre, frequentemente, nei pazienti ricoverati per altre patologie, lo SC rappresenta un problema medico coesistente che ne complica notevolmente la gestione e la prognosi. ${ }^{4}$ Un recente studio FADOI condotto in $23 \mathrm{UO}$ di Medicina Interna (MI) campane ha dimostrato che l'età avanzata e la presenza di molteplici comorbilità caratterizzano i pazienti con SC ricoverati nei reparti di MI. ${ }^{5}$ Bisogna considerare che oltre il $50 \%$ dei casi di SC interessa la popolazione con età $\geq 75$ anni, fascia d'età in continuo incremento anche in Campania $(>1 / 3$ della popolazione generale residente). Infatti, le differenze demografiche evidenziate in un altro studio

Corrispondente: Fernando Gallucci, UOC Medicina Interna 3 , AORN A. Cardarelli, via A. Cardarelli 9, 80131 Napoli, Italia. E-mail: fernando.gallucci@aocardarelli.it

Articolo pubblicato secondo la Creative Commons Attribution NonCommercial 4.0 License (CC BY-NC 4.0).

${ }^{\circ}$ Copyright: the Author(s), 2021

Licensee PAGEPress, Italy

QUADERNI - Italian Journal of Medicine 2021; 9(2):e17
FADOI di confronto Toscana-Campania (TUSCAM), che vedevano la popolazione campana rappresentata da pazienti di età inferiore, vanno progressivamente riducendosi nel tempo. ${ }^{6}$

Anche nella nostra Regione, il sistema ospedaliero non è in grado e non deve, da solo, garantire una risposta all'insieme dei fabbisogni assistenziali dei pazienti con SC: è necessario organizzare ed ottimizzare una filiera complessiva per affrontare la prevenzione e le varie forme di terapia nello $\mathrm{SC}$, la riabilitazione cardiovascolare, le forme di gestione alternative al ricorso al pronto soccorso ed al ricovero ordinario, fino alla fase delle cure palliative. ${ }^{7}$

I dati del Programma Nazionale Esiti dell'AGENAS (aggiornamento 2018) mostrano che in Campania, in media, la frequenza di ospedalizzazioni per SC è elevata, e seppure i dati di mortalità per questa patologia entro i 30 giorni dalla dimissione siano in media con il dato nazionale, la percentuale di riammissione in tale intervallo di tempo (fase vulnerabile) è pari a circa il $14 \%$, con grande eterogeneità tra le province. Il dato è confermato dallo studio Campania Scompenso (CASCO) dell'ANMCO regionale. ${ }^{8}$ Relativamente alla gestione di pazienti con $\mathrm{SC}$, una survey ANMCO Campania (Dati presentati al Congresso nazionale ANMCO 2015), condotta su un campione di Medici di Medicina Generale (MMG), mostra che la Medicina Generale (MG) giudica favorevolmente la costituzione di una rete per la gestione integrata dello $\mathrm{SC}$ che ruoti intorno ad Unità SC, al cui funzionamento i MMG ritengono di poter contribuire. Il mancato coordinamento ASL-Ospedali viene percepito quale principale problema gestionale, evidenziando, tra l'altro, che la rete ambulatoriale della Cardiologia ospedaliera per valutazioni diagnostico-terapeutiche urgenti o per servizi in DH terapeutico è raramente fruibile sul territorio con conseguente carenza di alternative al ricorso al PS anche per quadri patologici non complessi o noti. ${ }^{8}$ Tra i fattori non cardiaci in grado di influenzare il numero di re-ospedalizzazioni per SC viene individuato anche il ruolo del Medico. A tal proposito, lo studio AGISCO, che ha raccolto i dati di pazienti ricoverati in 29 UO di MI campane associate FADOI, ha dimostrato che ancora persiste un'a- 
derenza non-ottimale alle più recenti linee guida prescrittive nei pazienti con SC. ${ }^{9}$

Nella regione Campania, sono stati realizzati progetti di Percorso Diagnostico Terapeutico Assistenziale (PDTA) sullo SC ed altri sono in fase di studio.

\section{Progetto PRESASCO}

L'ASL della provincia di Caserta ha attivato un progetto, inserito nel programma Terra dei Fuochilinea 3 per il trattamento precoce dello SC che prevede il coinvolgimento di oltre $50 \mathrm{MMG}$ ed i reparti di Cardiologia di alcuni PO. Viene condivisa una scheda clinico/anamnestica compilata dai MMG che intercettano casi di SC con relativo avvio agli ambulatori dedicati, istituiti presso le UO di Cardiologia dell'ASL, mentre gli specialisti Cardiologi delle suddette UO compilano una scheda di dimissione che viene acquisita in tempo reale dai MMG. Le riunioni, tenutesi a partire dal novembre 2018 , hanno visto impegnate varie categorie professionali (rappresentanti della MG, delle UO di Cardiologia dei Presidi della Asl di Caserta e del Servizio Controllo di GestioneStruttura Saniarp), con l'obiettivo di condividere un percorso finalizzato all'individuazione di pazienti scompensati e di costruirne uno virtuoso in grado di garantire al paziente il più rapido accesso alle cure ed il monitoraggio costante. Alla piattaforma Saniarp è stato chiesto di creare una scheda ad hoc sul paziente scompensato, in base alle indicazioni fornite dagli stessi medici, per facilitare la comunicazione fra la Medicina di base e l'Ospedale. I rappresentanti dei MMG hanno dato la disponibilità ad implementare $\mathrm{i}$ dati relativi ai pazienti scompensati su Saniarp, cominciando da quelli in classe $\mathrm{C}$ e $\mathrm{D}$, che risultano più gravi e con più di una ospedalizzazione per SC. I rappresentanti dei Medici ospedalieri presenti hanno dato disponibilità ad organizzare ambulatori dedicati in giorni ed orari stabiliti, prenotati direttamente dal Medico di base tramite Saniarp che elaborerà la lista di attesa dei suddetti ambulatori.

\section{PDTA ASL NA 1}

Anche l'ASL NA1 ha provveduto all'elaborazione di un PDTA con l'impegno di darne diffusione a tutte le figure professionali coinvolte nei processi assistenziali e di procedere ad un censimento aziendale delle Strutture da coinvolgere in un'esperienza pilota per testarne la piena operatività. Inoltre, è stata considerata la necessità di provvedere alla formazione continua del personale da coinvolgere, con l'organizzazione di Master di I, II Livello e di Audit Clinici sullo SC.

\section{Progetto GPS: gestione multidisciplinare del paziente con scompenso cronico}

Il progetto, portato avanti sotto il coordinamento degli Organi regionali, è finalizzato ad individuare una linea guida regionale per la Gestione Integrata in Rete dello SC ed a condividere l'importanza della sensibilizzazione e del coinvolgimento di tutti gli attori, la proposta di percorso di mobilizzazione del paziente tra figure professionali, un PDTA del paziente con SC cronico e gli indicatori di processo e di esito del percorso di gestione (Tabella 1 e Figura 1). Nell'ambito del Piano Nazionale della Cronicità, l'obiettivo generale della gestione di pazienti con $\mathrm{SC}$ cronico è prevenire le riacutizzazioni e la progressione della

Tabella 1. Progetto GPS: Obiettivi di fondo della gestione integrata.

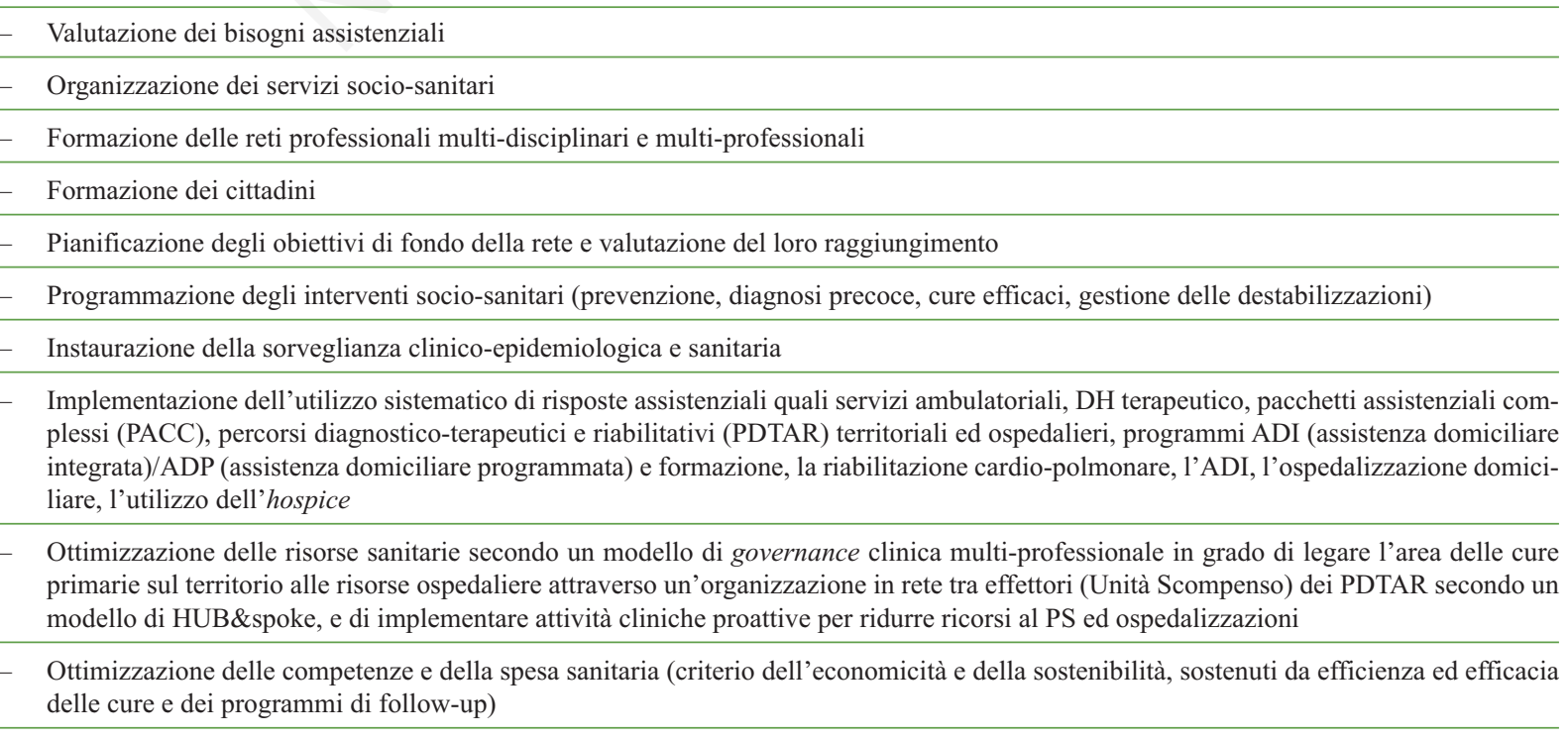




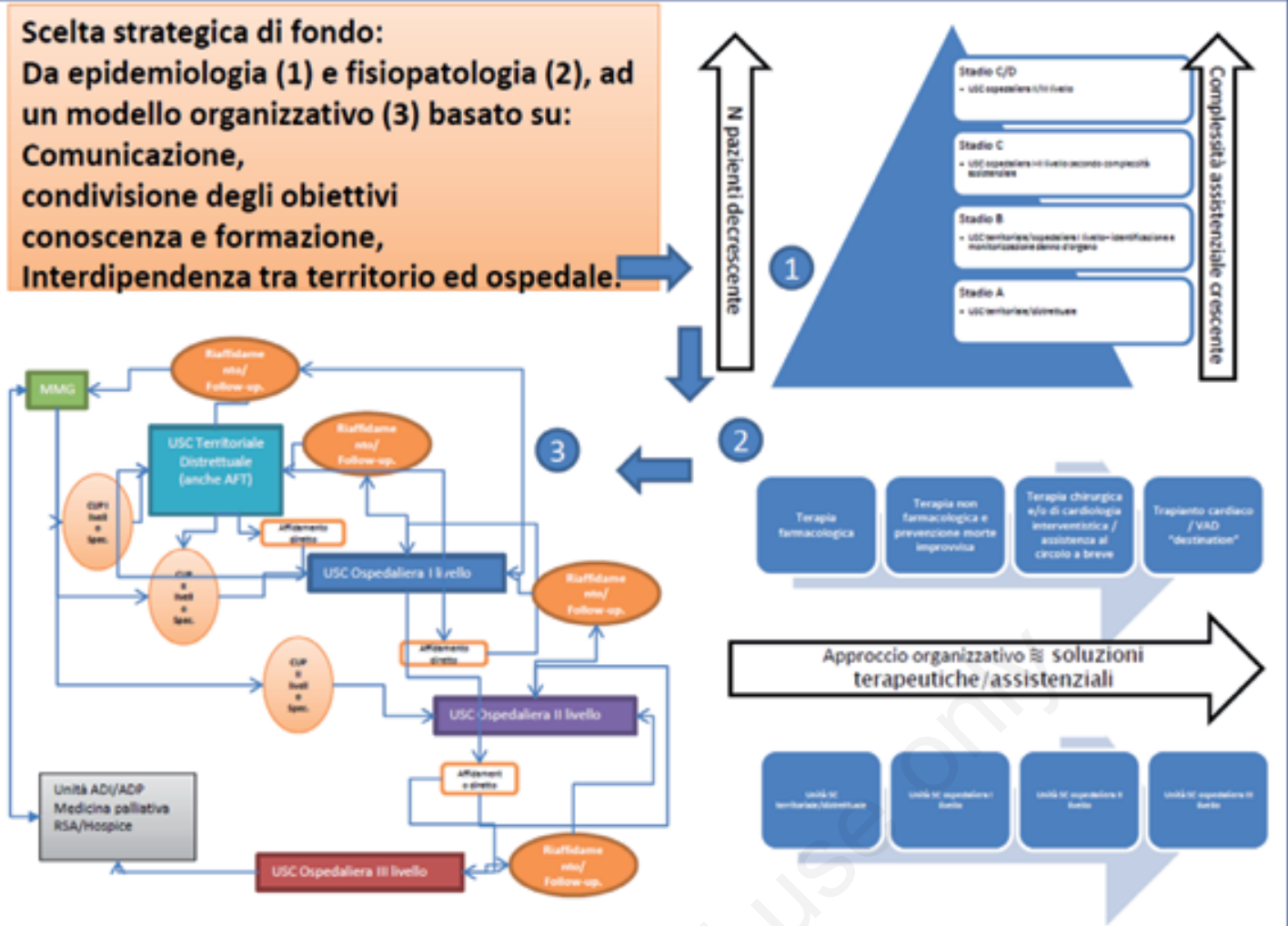

Tratto da: Linea d'indirizzo clinico-organizzativo della regione Campania, 2020.

Figura 1. Proposta di modello organizzativo. Tratto da: Linea d'indirizzo clinico-organizzativo della regione Campania, 2020. Documento presentato alla Commissione Permanente Sanità Regione Campania (Novembre 2015).

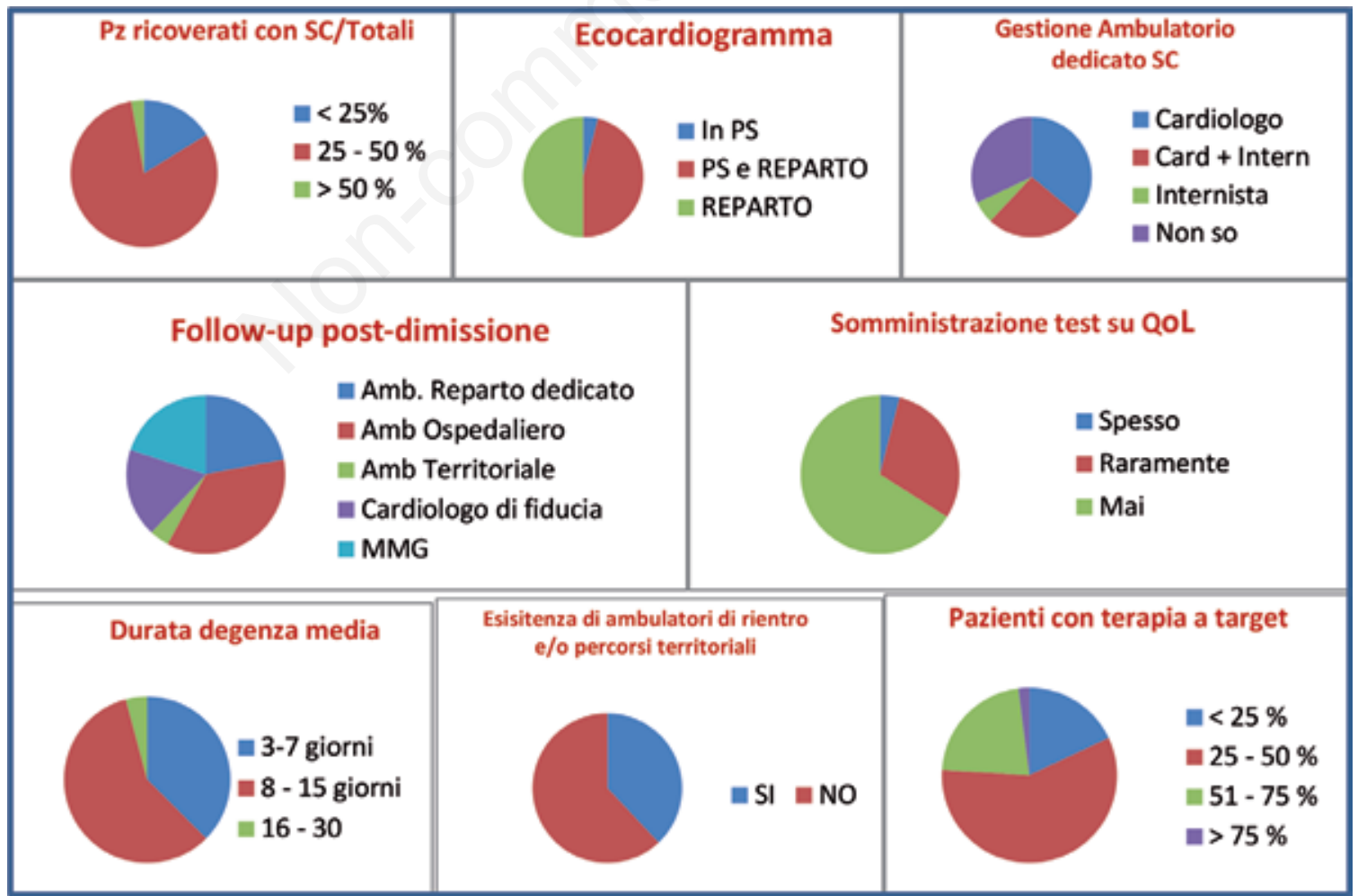

Figura 2. Dati dalla Indagine conoscitiva gestione Scompenso Cardiaco in Medicina Interna - FADOI CAMPANIA (Ottobre 2020). 
malattia con una gestione attiva ed intensiva del paziente nel suo domicilio, evitando ospedalizzazioni inappropriate. Costruire un modello organizzativo centrato sul paziente è garanzia di effettiva presa in carico dei bisogni globali, di creazione di una relazione empatica tra il team assistenziale e la persona con cronicità ed i suoi Caregiver di riferimento.

I due workshops, organizzati in occasione del Progetto GPS sul tema (20.06.2018 e 29.10.2019), hanno offerto l'opportunità di interessanti confronti attraverso la condivisione del lavoro del gruppo multidisciplinare finalizzato all'individuazione della proposta di gestione della specifica tipologia di pazienti per valorizzare il modello di governance, tracciabilità ed innovazione del processo identificato anche con il supporto delle analisi estratte dalla piattaforma Saniarp.

Nell'ottobre 2020, è stata condotta una Survey per conto dell'area SC del Direttivo Regionale FADOI che ha realizzato una fotografia della attuale situazione logistico-assistenziale relativa alla gestione del paziente con SC in Campania (Figura 2).

Risulta, quindi, evidente il grande impegno in atto nella nostra Regione per la progettazione di programmi gestionali relativi al paziente affetto da SC, patologia caratterizzata da notevole impatto socio-sanitario.

\section{Bibliografia}

1. Catapano A, Vanuzzo D. Epidemiologia Nazionale e Regionale. G Ital Cardiol 2009; 10:38S-57S.
2. Ministero della Salute. Rapporto Annuale sull'attività di ricovero ospedaliero. Dati SDO 2013. Available from: http://www.salute.gov.it/imgs/C_17_pubblicazioni_219 0 allegato.pdf.

3. Biagi P, Gussoni G, Iori I, et al. CONFINE Study Group; Clinical profile and predictors of in-hospital outcome in patients with heart failure: the FADOI CONFINE Study. Int J Cardiol 2011;152:88-94.

4. Cavaliere R, Gallucci F, Mathieu G. La prevenzione cardiovascolare nello Scompenso Cardiaco, in: Position paper FADOI sulla prevenzione cardiovascolare nei pazienti complessi a rischio. Quaderni IJM 2015;3:339-55.

5. Gallucci F, Fontanella A, Ronga I, Uomo G, et FASHION Study Group. Results of prospective multicenter study on heart failure on Campania Internal Medicine wards: the FASHION study. IJM 2017;11:184-190.

6. Gallucci F, Biagi P, Landini G, et al. Final report of Tuscany-Campania heart failure study. XXI Congresso Nazionale della Società Scientifica FADOI. Roma, 14-17 maggio 2016. IJM 2016;10:S50.

7. Aspromonte N, Gulizia MM, Di Lenarda A, et al.. La rete cardiologica per la cura del malato con scompenso cardiaco: organizzazione dell'assistenza ambulatoriale. G Ital Cardiol (Rome) 2016;17:570-93.

8. Palmieri V, Iodice P, Palermo A, et al. Prevenzione e sorveglianza attiva nello scompenso cardiaco: ANMCO Campania per una sinergia sistemica ospedale-territorio. Cardiologia negli Ospedali 2019;227:58-62.

9. Gallucci F, Ferrara L, Mastrobuoni C, et al. A.S.G (Agisco Study Group). Adherence to guidelines recommandations for heart failure treatment: data from a multicenter observational study (AGISCO study). XII Congresso Nazionale della Società Scientifica FADOI, Sorrento 13 -16 maggio 2017. IJM 2017;11:S9. 


\title{
Modelli a confronto nei percorsi gestionali dello scompenso cardiaco: l'esperienza pugliese
}

\author{
Franco Mastroianni, ${ }^{1}$ Giovanni Larizza, ${ }^{1}$ Fabiana D’Onofrio, ${ }^{1}$ Anna Belfiore, ${ }^{2}$ Maria Veronica Palma, ${ }^{2}$ Simona Cataldi ${ }^{2}$
}

${ }^{1}$ UOC Medicina Interna, EE Ospedale Generale Regionale F. Miulli, Acquaviva delle Fonti (BA); ${ }^{2}$ UOC Medicina Interna Universitaria A. Murri, Policlinico, Bari, Italia

\section{Introduzione}

Lo scompenso cardiaco cronico (SCC) è un problema sanitario tra i più rilevanti nei paesi industrializzati. In Italia rappresenta la prima causa di ricovero dopo i 65 anni con un notevole impegno economico per il SSN. L'età avanzata e le multiple comorbilità dei pazienti affetti da SCC, condiziona frequentemente il ricovero di questi pazienti ricoveri presso le unità di Medicina Interna, dove il DRG 127 è una dei più comuni. L'EURO (Observational Research Program Heart Failure Pilot Survey) ha riportato un tasso di reospedalizzazioni ad un anno del $43,9 \%$ e del $31,9 \%$ rispettivamente per i pazienti ammessi in ospedale per scompenso acuto e cronico.

In Puglia sono registrati più di 80.000 pazienti con SCC e circa 15.000 ricoveri anno con un incremento negli ultimi anni ed una significativa quota di ricoveri ripetuti $(30 \%)$. La fascia più interessata è quella di età superiore ai 75 anni. I pazienti anziani con SCC hanno frequenti riacutizzazioni che possono essere variamenti associati a fattori precipitanti (bronchite intercorrente, insorgenza di fibrillazione atriale, scompenso diabetico) $\mathrm{o}$ ad un trattamento inadeguato, multiple comorbidità, mancanza di un adeguato supporto sociale, gestione non ottimale nel periodo extra-ospedaliero. In assenza di programmi di continuità assistenziale, i pazienti fanno riferimento ad una nuova ospedalizzazione per risolvere problemi che potrebbero essere trattati in altri setting assistenziali. Vengono di seguito riportate le esperienze

Corrispondente: Franco Mastroianni, UOC Medicina Interna, EE Ospedale Generale Regionale F. Miulli, SP per Santeramo, $\mathrm{Km} 4,100$, Acquaviva delle Fonti (BA), Italia.

E-mail: franco_mastroianni@libero.it

Articolo pubblicato secondo la Creative Commons Attribution NonCommercial 4.0 License (CC BY-NC 4.0).

${ }^{\circ}$ Copyright: the Author(s), 2021

Licensee PAGEPress, Italy

QUADERNI - Italian Journal of Medicine 2021; 9(2):e18 condotte in due ospedali pugliesi, dove sono stati attivati ambulatori per lo SCC presso Unità Operative di Medicina Interna.

\section{L'esperienza dell'ambulatorio Miulli}

Presso l'Ospedale F. Miulli è attivo un ambulatorio internistico sullo scompenso cardiaco, afferente dapprima alla geriatria e poi alla medicina interna, che è stato realizzato a partire dal progetto INNESCO (INnovazione NEllo Scompenso $)^{1}$ che ha valutato gli effetti di un programma educazionale condotto su pazienti e caregiver al fine di ridurre le ri-ospedalizzazioni. Lo studio INNESCO, sebbene preliminare e con un numero ridotto di pazienti, ha dimostrato che in seguito alla presa in carico del paziente da parte di un case manager (infermiere) si ottiene una riduzione delle ospedalizzazioni. Il progetto prevedeva la somministrazione di un questionario per il rilevamento dei bisogni e la successiva consegna di un breve vademecum per la gestione domiciliare comprensivo di un diario giornaliero di automonitoraggio. Alla fine del progetto, della durata di sei mesi, è stato somministrato un questionario per verificare le capacità di apprendimento del percorso educazionale da parte dei pazienti e del caregiver principale. I 30 soggetti studiati (età media 77.5 anni) erano equamente distribuiti nelle 4 classi NYHA e solo il 10\% conosceva i sintomi della propria malattia.

Lo studio INNESCO ha evidenziato come il paziente inizialmente sia abbastanza disinformato delle caratteristiche generali dello scompenso. L'ignoto determina disinteresse, scarsa aderenza alla terapia e di conseguenza aumento delle riacutizzazioni e dei ricoveri ospedalieri.

L'educazione terapeutica fornita al paziente ha permesso non solo di conoscere le caratteristiche della malattia, dei sintomi e segni, della terapia farmacologica, ma soprattutto di migliorare la qualità della sua vita. Alla fine del percorso educazionale emerge un netto miglioramento delle conoscenze del paziente ( $18 \mathrm{pz}$ su 30 conoscono la malattia rispetto a 6 precedenti), che ha 
determinato un aumento della compliance alla terapia farmacologica ed un aumento complessivo dell'empowerment. Ruolo essenziale è stato quello dell'infermiere, il principale responsabile dell'attività di gestione integrata. Per le sue caratteristiche professionali, e se specificatamente formato, risulta particolarmente indicato nei programmi di counselling del paziente e dei familiari e nell'educazione sanitaria. Infine il dato sui ricoveri: il progetto ha determinato una riduzione delle re-ospedalizzazioni complessiva del $52 \%$.

\section{L'esperienza della Clinica Medica Universitaria A. Murri, Policlinico, Bari}

Presso la divisione di Medicina Interna Universitaria A. Murri, Policlinico di Bari, è attivo da diversi anni un ambulatorio per lo scompenso cardiaco, con l'obiettivo di assicurare la continuità assistenziale per il pazienti con SC dimessi dalla stessa unità operativa o da altre medicine. L'ambulatorio è gestito da internisti con competenze cardiologiche, e supportato dalla presenza di una infermiera professionale. Punto di partenza è l'inquadramento diagnostico-terapeutico effettuato durante il ricovero ospedaliero, che prevede lo screening bioumorale con dosaggio di NT-pro-BNP, esame elettrocardiografico ed ecocardiografico, l'attribuzione della classe funzionale NYHA, nonché la rivalutazione della terapia medica come indicato dalle linee guida. Alla dimissione è previsto un colloquio con il paziente e i parenti per valutare la consapevolezza del paziente relativa alla patologia cardiaca e per eventuali consigli sullo stile di vita ed automonitoraggio del peso, della pressione arteriosa e degli eventuali edemi declivi. Il paziente viene rimandato in ambulatorio dopo 15-30 giorni dalla dimissione per valutare la stabilità clinica e l'aderenza alla terapia prescritta; lo stesso viene quindi inserito in un protocollo di gestione ambulatoriale che prevede visite programmate ogni 3-6 mesi. Sono possibili visite non programmate, su segnalazione del medico di medicina generale o dello stesso paziente, se vengono rilevati sintomi di allarme: accentuazione della dispnea, comparsa di edemi declivi e incremento ponderale, valori pressori elevati o $<90 \mathrm{mmHg}$, episodi sincopali. Durante ciascuna visita vengono raccolte informazioni relative a segni e sintomi dello SC, adesione alla terapia prescritta, supporto sociale, eseguito un elettrocardiogramma e rivalutata la classe NYHA; ogni 6-12 mesi si ripete l'ecocardiogramma. Ogni 3-6 mesi vengono prescritti esami biuomorali. La terapia medica è rivalutata ad ogni controllo, in particolare viene effettuata la titolazione dei $\beta$-bloccanti e degli ACE-inibitori $\mathrm{o} /$ sartani e viene rivalutato il dosaggio dei diuretici. In considerazione dell'età avanzata dei pazienti che afferiscono all'ambulatorio scompenso e delle multiple co-morbidità, viene effettuato il controllo delle patologie concomitanti di interesse internistico, quali diabete, patologie tiroidee, anemie carenziali, ipertensione, insufficienza renale, disturbi osteo-articolari. Al termine di ciascuna visita viene fornita al paziente una lettera per il curante, in modo da assicurare la continuità assistenziale fra la struttura ospedaliera e il territorio. Se vengono ravvisate situazioni di emergenza il paziente viene indirizzato ad un nuovo ricovero ospedaliero. Il contatto continuo con il paziente è assicurato da una linea telefonica messa a disposizione per le urgenze e per eventuali consigli terapeutici e sullo stile di vita. Recentemente è stata effettuata una rivalutazione dell'attività ambulatoriale valutando gli esiti del programma di assistenza a lungo termine ( 2 anni) su un gruppo di 147 pazienti. ${ }^{2}$ I pazienti arruolati nella valutazione (età media 76 $\pm 9,6$ anni, con il $38,8 \%$ di età superiore a 80 anni), avevano una elevata prevalenza di comorbidità e la maggior parte era in classe NYHA III-IV. Le cause più comuni di SC erano l'ipertensione e le valvulopatie. Più frequentemente il pazienti avevano uno $\mathrm{SC}$ a frazione d'eiezione conservata $(61,9 \%)$. Nel corso del followup è stato osservato un significativo miglioramento della classe NYHA a 6 mesi ed una stabilizzazione della stessa a 24 mesi. Questo dato correla con l'ottimizzazione della terapia medica prescritta secondo le raccomandazioni delle linee guida internazionali. In particolare si è registrato un incremento dell'uso dei farmaci che agiscono a livello del sistema renina-angiotensina (ACE-inibitori e sartani), dei $\beta$-bloccanti e dei diuretici, compresi i risparmiatori di potassio. Il dato più significativo dello studio osservazionale è stata la riduzione delle ospedalizzazioni per SC del $42 \%$ nei 24 mesi di osservazione, ottenuta dal confronto con le ospedalizzazioni degli stessi pazienti nei 24 mesi precedenti lo studio, quando non erano inseriti in alcun programma di gestione ambulatoriale. I principali fattori che hanno inciso sulle re-ospedalizzazione, in accordo con altri dati della letteratura, sono stati l'anemia, l'appartenenza al classe NYHA III-IV e la precedente ospedalizzazione.

\section{Bibliografia}

1. Dell'era P, Pietroforte C, Mastroianni F, et al. Management of heart failure in outpatients: INNESCO study. Ital J Med 2014;8(suppl. 2):May.

2. Belfiore A, Palmieri VO, Di Gennaro C, et al. Long term management of chronic heart failure patients in internal medicine. Intern Emerg Med 2020;15:49-58. 


\title{
Modelli a confronto nei percorsi gestionali dello scompenso cardiaco: l'esperienza di Sassari. Il progetto LEAP
}

\author{
Carlo Andrea Usai, Francesco Luigi Bandiera \\ Medicina Interna, Ospedale Santissima Annunziata, Azienda Ospedaliero-Universitaria, Sassari, Italia
}

\section{Introduzione}

Lo scompenso cardiaco (SC) è una delle patologie croniche più diffuse nei Paesi avanzati, con un elevato impatto sulla qualità della vita, la sopravvivenza (mortalità del 50\% a 5 anni dalla diagnosi - paragonabile a quello delle neoplasie maligne) ed il consumo di risorse per il sistema sanitario. ${ }^{1,2}$

Nei Paesi Occidentali la prevalenza dello SC è dell' $1-2 \%$ nella popolazione generale con picchi del $6,4 \%$ nei soggetti con età $>65$ anni. Il ricovero per SC (DRG 127) rappresenta la seconda causa di ricovero dopo il parto e la prima causa di ospedalizzazione dei pazienti di età superiore ai 65 anni. $^{2}$

\section{Criteri e strumenti di ricerca}

\section{Obiettivi e metodologia}

Il progetto complessivo di riorganizzazione della gestione del paziente con scompenso cardiaco si articola nelle tre fasi di seguito riportate: ${ }^{3-4}$

- Analisi. Analisi delle attuali modalità di presa in carico dei pazienti con SC nei principali reparti di afferenza del paziente (Cardiologia, Medicina interna, Geriatria Clinica Medica e Pronto Soccorso), nei percorsi ambulatoriali dedicati e dei volumi di pazienti gestiti dalla struttura.

- Piano di intervento. Identificazione, valutazione e validazione di un piano di interventi di leve di mi-

Corrispondente: Carlo Andrea Usai, Medicina Interna, Ospedale Santissima Annunziata, Azienda Ospedaliero-Universitaria, via Enrico De Nicola 1, 07100 Sassari, Italia. Tel.: +39.0792061606. E-mail: carlo.usai@aousassari.it

Articolo pubblicato secondo la Creative Commons Attribution NonCommercial 4.0 License (CC BY-NC 4.0).

${ }^{\circ}$ Copyright: the Author(s), 2021

Licensee PAGEPress, Italy

QUADERNI - Italian Journal of Medicine 2021; 9(2):e19 glioramento potenzialmente attivabili per l'ottimizzazione della presa in carico.

- Implementazione. Programmazione delle attività utili al fine di garantire l'effettiva implementazione delle soluzioni di miglioramento selezionate, attraverso l'identificazione dei responsabili dei singoli interventi, la definizione della stima dei tempi e il monitoraggio dell'avanzamento delle attività.

La realizzazione di tale progetto è stata resa possibile dalla costituzione di un gruppo di lavoro multidisciplinare aziendale (internisti, cardiologi, geriatri, medici del Pronto Soccorso) che ha curato la stesura del presente documento. Bip Life Sciences ha supportato il gruppo nell'intero percorso progettuale assicurando la collaborazione di personale qualificato con esperienza nell'ottimizzazione organizzativo-gestionale dei percorsi paziente.

\section{L'analisi del percorso paziente}

L'analisi dell'attuale percorso di riferimento del paziente è stata condotta ricostruendo la modalità di presa in carico nelle seguenti fasi: i) accesso in Pronto Soccorso (Figura 1) e trattamento nei Reparti di degenza (Figura 2); ii) follow-up postdimissione.

\section{Il nuovo indirizzo del paziente con scompenso cardiaco}

Sulla base delle evidenze raccolte, il gruppo di lavoro ha identificato i seguenti ambiti di intervento prioritari: i) la definizione di standard condivisi per la presa in carico in PS; ii) la definizione di standard condivisi per l'invio ai reparti di ricovero (Figura 3); iii) l'adozione di una lettera di dimissione condivisa e standardizzata quale efficace strumento di raccordo col territorio (MMG) e di promozione della continuità di cure; iv) l'articolazione degli attuali ambulatori al fine di offrire una continuità assistenziale, con percorsi di cura differenziati, personalizzati, omogenei. 


\section{Bip.

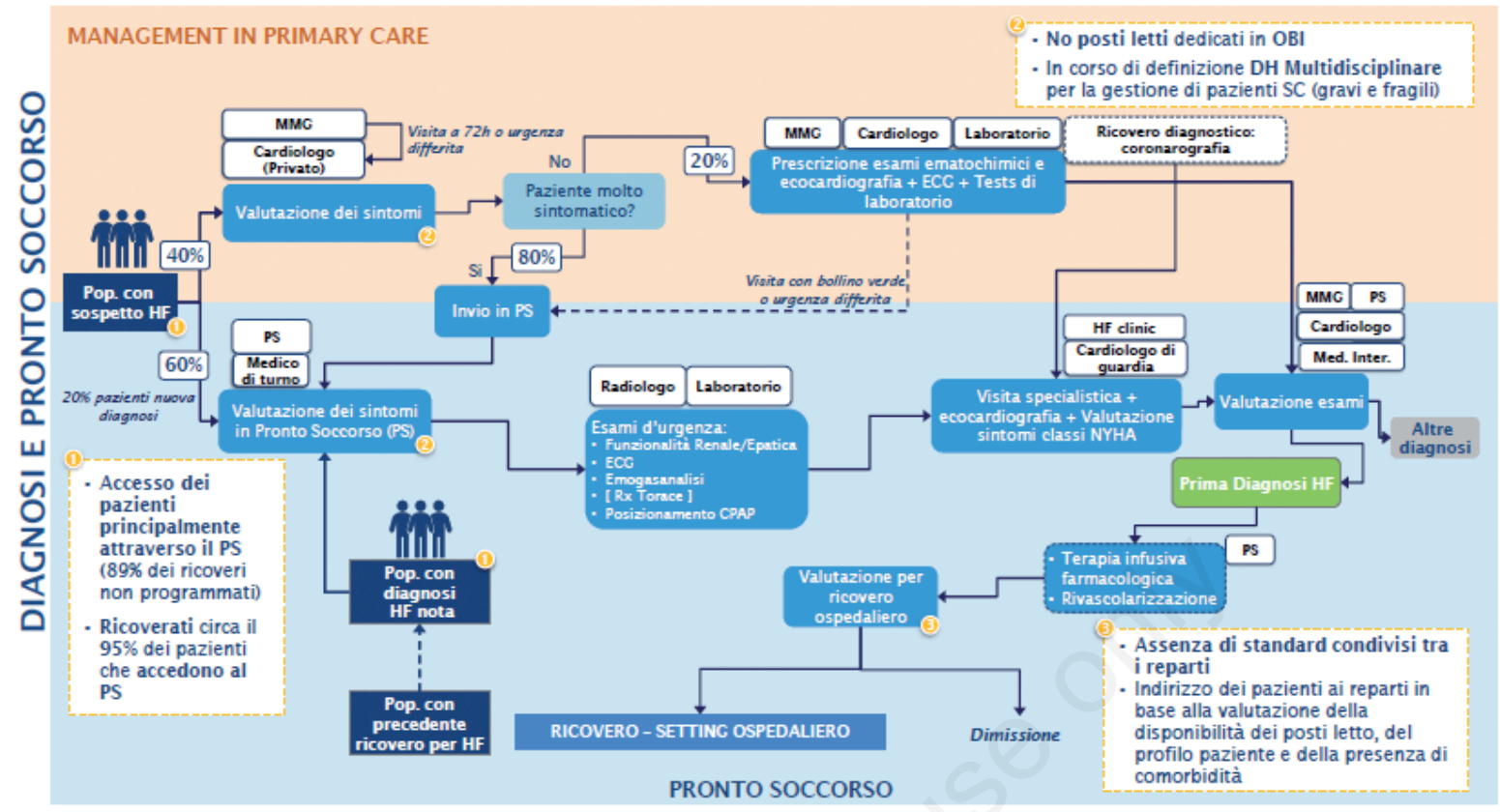

Q. Principali snodi organizzativi del Patient Flow

Nota: Ricostruzione del Patient Flow attraverso le interviste ai clinici coinvolti nel progetto (Novembre 2018)

Figura 1. Il percorso SC: accesso in Pronto Soccorso.

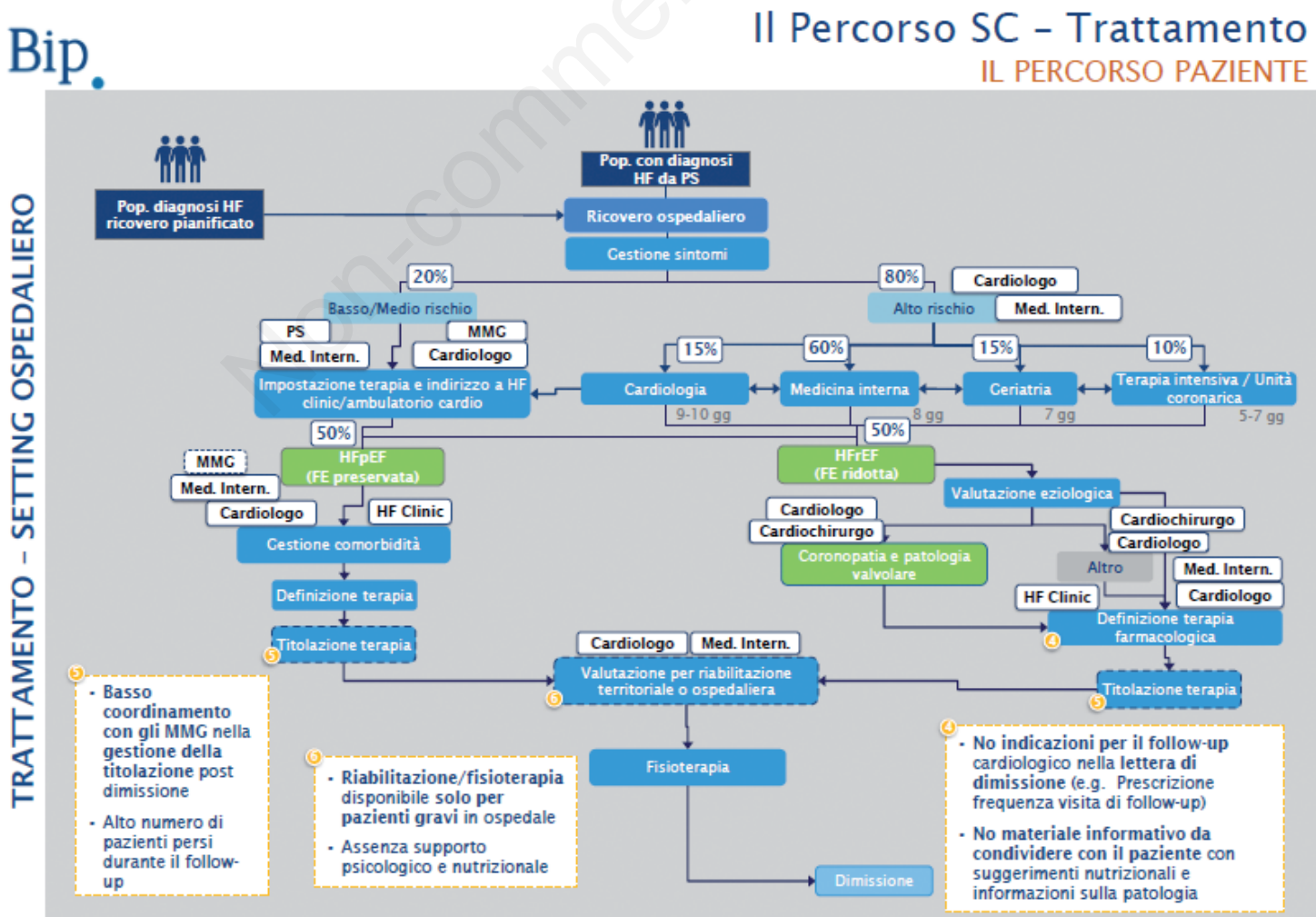

Figura 2. Il percorso SC: trattamento, setting ospedaliero. 


\section{Criteri di Indirizzo dal PS ai Reparti}

\begin{tabular}{l} 
PROFILO CLINICO PAZIENTE CON SC \\
PRIMO EVENTO SC \\
Classe NYHA I-II-III scatenato da evento concomitante (infettivo o altro) \\
extra cardiaco $\mathrm{e} / \mathrm{O}$ : \\
1. Aritmico che necessitano di monitoraggio in ambiente intensivo \\
2. Aritmico non grave \\
Classe NYHA > III richiede trattamento intensivo e studio emodinamico \\
\hline SC CRONICO \\
Aritmie a rischio o portatore di ICD che ha scaricato correttamente \\
SC dx da ipertensione polmonare in studio pneumologico/reumatologico \\
SC di origine ischemica acuta (SCA) \\
\hline RECIDIVA SC \\
Paziente in attesa di trattamento interventistico - chirurgico \\
Cardiopatia in fase molto avanzata che richieda trattamento intensivo, se \\
ipotizzabili strategie di supporto ventricolare - trapianto \\
Cardiopatia e peggioramento della classe funzionale senza EPA o shock \\
con patologia che richieda approfondimento o terapia \\
Grave patologia associata SC anche in classe funzionale avanzata, con \\
prognosi infausta a breve per patologia o con controindicazioni ad \\
indagini emodinamiche \\
SC e sindrome coronarica acuta (STEMI, NSTEMI, angina instabile)
\end{tabular}

2010 vo Ruciness Intenration Partners S n A

Figura 3. Criteri di indirizzo dal Pronto Soccorso ai reparti.

\section{Bibliografia}

1. Ponikowski, et al. 2016 ESC Guidelines for the diagnosis and treatment of acute and chronic heart failure. Eur Heart J 2016;37:2129-200.

2. Aspromonte, Golizia, Di Lenarda, et al. Documento di consenso ANMCO/SIC. La rete cardiologica per la cura del malato con scompenso cardiaco organizzazione dell-assistenza ambulatoriale. G Ital Cardiol 2016;17:570-93.

3. Marangoni, et al. Epidemiologia, impatto organizzativo e costi dello scompenso cardiaco in Italia. G Ital Cardiol 2012;13:139S-44S.

4. Ministero della Salute. Piano Nazionale della Cronicità; 2016. 


\title{
Il ruolo delle cure palliative nello scompenso cardiaco end-stage
}

\author{
Claudio Santini, ${ }^{1}$ Maria Serena Fiore ${ }^{2}$ \\ ${ }^{1}$ Medicina Interna, Ospedale Vannini, Roma; ${ }^{2}$ Medicina Interna, Ospedale Pertini, Roma, Italia
}

\section{Introduzione}

Il ruolo delle cure palliative nelle patologie non oncologiche è da alcuni anni al centro dell'interesse della comunità scientifica per motivi che attengono alla sfera clinico-terapeutica, deontologica, sociale ed anche economica. Il tema è ovviamente in stretta connessione al concetto di terminalità della quale, in realtà si parla da molti anni. Già ne Il Caleidoscopio edito a Genova nel 1987 dedicato a Il paziente terminale si legge: il tema è interessante ed altrettanto apertamente trascurato nel corso della formazione del medico. I problemi che infatti pone il malato terminale non sono solo strettamente sanitari ma anche psicologici, deontologici, sociali ed umani. ...Ma se si vogliono identificare i pazienti da definire in fase terminale di malattia, bisogna riconoscere che anche da questo punto di vista non esiste una sempre facile separazione del periodo in cui l'individuo continua a vivere, sia pure sotto il peso della malattia e delle sue limitazioni, dalla fase in cui il processo si fa rapidamente evolutivo e la fine compare. ${ }^{1}$

Il dibattito è per molti aspetti ancora aperto anche se esistono ormai definizioni ufficiali. Dal Glossario di riferimento in cure palliative (2003): per malato in fase terminale si intende la persona affetta da una patologia cronica evolutiva in fase avanzata, per la quale non esistano o siano sproporzionate terapie con l'obiettivo di una stabilizzazione della malattia e/o di un prolungamento significativo della vita. Questa fase viene stabilita indicativamente con un criterio temporale in novanta giorni per il paziente oncologico mentre per le patologie croniche degenerative non oncologiche è necessario individuare altri criteri, non necessariamente di durata della vita residua.

Corrispondente: Claudio Santini, Medicina Interna, Ospedale Vannini, Via Acqua Bullicante 4, 00177 Roma, Italia. E-mail: santini.cla@gmail.com

Articolo pubblicato secondo la Creative Commons Attribution NonCommercial 4.0 License (CC BY-NC 4.0).

${ }^{\circ}$ Copyright: the Author(s), 2021

Licensee PAGEPress, Italy

QUADERNI - Italian Journal of Medicine 2021; 9(2):e20
Tra le tante affermazioni rispetto al tema possiamo condividere: che la terminalità si può identificare con una fase temporale ma soprattutto determina un percorso che deve tenere conto di molteplici fattori biologici, clinici, etici, psicologici, sociali; che sono stati individuati molti indicatori ma ciascuno di essi preso singolarmente ha scarso valore e che, pertanto, nella definizione della terminalità, occorre procedere a una valutazione multidimensionale. ${ }^{2-4}$

A lato di queste considerazioni, si colloca la raccomandazione dell'Organizzazione Mondiale della Sanità per cui le Cure palliative dovrebbero essere erogate il più precocemente possibile nel corso di una malattia cronica, quando cioè i malati si avvicinano alla fase finale della vita, fino alla fase terminale.

Nel 2010 il Medical Council elabora un documento sul Fine vita in cui si definisce che I malati si avvicinano alla fine della vita quando è probabile che essi muoiano entro i successivi 12 mesi. In questa definizione sono inclusi i malati la cui morte è imminente (attesa entro poche ore o giorni) e quelli con malattie inguaribili, progressive, in fase avanzata, con una condizione clinica di fragilità generale. Questi malati sono a rischio di morte sia per una crisi acuta e improvvisa legata alla loro malattia cronica, sia per condizioni acute causate da eventi improvvisi e catastrofici. ${ }^{5,6}$

Nel Dicembre 2011, in Gran Bretagna, il National Institute for Health and Clinical Excellence (NICE) auspica lo sviluppo di Cure palliative totalmente orientate ai bisogni/preferenze dei malati che si avvicinano alla fine della vita e dei loro familiari e sempre meno dipendenti dalla tipologia della diagnosi. ${ }^{7}$

Nonostante i vari ed autorevoli tentativi di chiarificazione il tema è tuttora costellato di domande e non sempre abbiamo risposte certe. ${ }^{4,5}$

La prima: Qual è il momento nel quale un malato può essere definito 'alla fine della vita'? Le possibili risposte sono ancora domande e tutte tra di loro interconnesse. L'imminenza della morte? Le preferenze e le aspettative del paziente? La gravità complessiva della malattia? La prognosi? Il giudizio clinico dei curanti? $?^{4-6}$

La seconda, cosiddetta sorprendente è cruciale ed almeno a questa dobbiamo trovare la nostra risposta: Quale dei pazienti che sto curando non sarei sorpreso 
se morisse entro ... 1 anno ${ }^{8,9}$ La risposta a questa domanda è infatti il punto di partenza per definire il percorso di ciascuno dei nostri pazienti perché nel caso in cui dovessimo decidere per il NO dovremmo andare a cercare indicatori generali di declino ed indicatori specifici per ciascuna patologia in un processo complesso di Identificazione (del paziente bisognoso di cure palliative, a maggior rischio di peggioramento e morte), Valutazione (dei bisogni e possibili supporti attraverso il colloquio con il paziente ed i suoi familiari), Pianificazione (di cure proattive, migliorando il coordinamento degli interventi e la comunicazione). ${ }^{9}, 10$

E ancora, la domanda del medico (che si sovrappone e fa eco a quella del paziente, della sua famiglia, dell'equipe... che si devono preparare): potevo prevedere come sarebbe andata a finire? Ho fatto davvero tutto quanto in mio potere per rendere gli ultimi attimi di vita del mio paziente più dignitosi? Il medico che non realizzi quanto poco tempo sia rimasto al paziente può perdere l'occasione di dedicare il tempo a migliorare la qualità della vita residua e negare al paziente l'opportunità di prepararsi alla morte. Può, in sintesi, perdere l'occasione per spostare il focus dalla malattia al paziente: e questo è lo scopo profondo delle cure palliative. ${ }^{10,11}$

Da qui la definizione di Cure palliative OMS 2014 un approccio che migliora la qualità della vita dei malati e delle loro famiglie che si trovano ad affrontare le problematiche associate a malattie inguaribili, attraverso la prevenzione e il sollievo della sofferenza per mezzo di una identificazione precoce e di un ottimale trattamento del dolore e delle altre problematiche di natura fisica, psicofisica e spirituale.

L'ultima domanda potrebbe essere quella del paziente, e citando Atul Gawande: ${ }^{12}$

Dal momento che le cure mediche sempre più al-

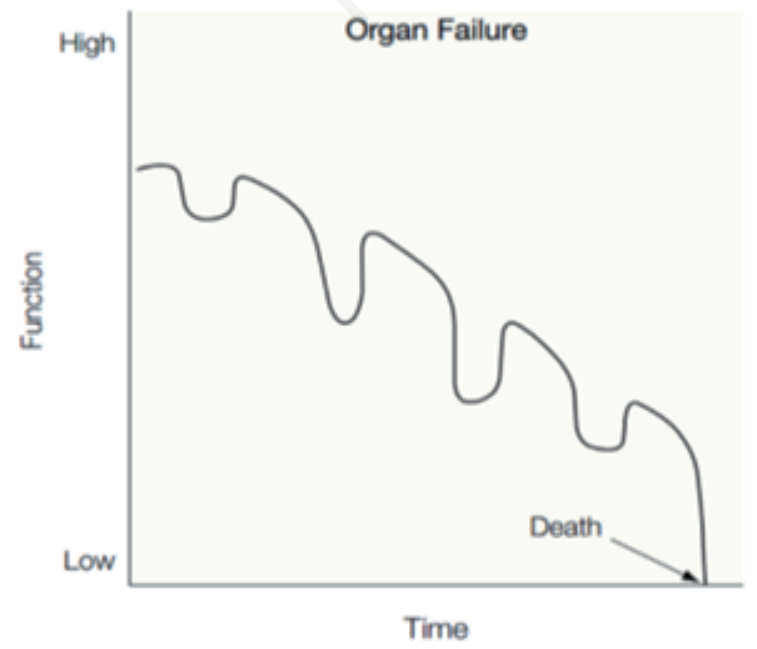

Figura 1. Traiettoria delle grandi insufficienze d'organo. l'avanguardia hanno portato a risolvere alcuni fondamentali problemi, come respirare (lo fa il ventilatore) o alimentarsi (ci pensano flebo o sondini), è sempre più difficile comprendere il timing del fine vita.

Come tu puoi decidere di esprimere i tuoi pensieri e le tue preoccupazioni riguardo alla morte, quando la medicina stessa ha reso quasi impossibile comprendere chi sono davvero le persone che sono vicine al momento della morte?

Tutto questo, è ormai chiaro, va valutato sia in ambito oncologico che non oncologico e pertanto anche nella gestione delle grandi insufficienze d'organo che sono di squisito interesse internistico. Facendo riferimento alle sole patologie cardiovascolari, l'OMS nel 2014 stima che le stesse costituiscano il $38,47 \%$ della necessità di cure palliative per adulti (essendo 34,01\% la quota per le patologie neoplastiche).

\section{Traiettorie di malattia ed insufficienza cardiaca}

Il problema della definizione della terminalità, soprattutto non oncologica, e del ruolo delle cure palliative nei diversi percorsi ha molto a che vedere con le ben note traiettorie di malattia. Differenziare tra le diverse traiettorie significa conoscere le relative necessità e contribuire a definire strategie personalizzate $\mathrm{e}$ migliori programmi di cura. ${ }^{13,14}$

La traiettoria dell'insufficienza cardiaca (IC) ha un tipico pattern in cui si alternano fasi di riacutizzazione a fasi di ripresa con un declino che si verifica progressivamente fino alla morte (entry-reentry) (Figura 1).

Tale andamento di malattia rende assai difficile decidere quando sia opportuno il tempo della cura palliativa. Infatti il medico, il paziente, la sua famiglia, l'equipe di assistenza sono abituati al fatto che dopo periodi di malattia anche molto grave il paziente possa anche drammaticamente migliorare e tornare ad una vita pressoché normale; ciò può portare ad una falsa prospettiva ed allontanare dalla consapevolezza del vicino declino. La prognosi, arte sempre incerta, è difficile da stimare se la malattia ha queste caratteristiche: quale sarà il peggioramento clinico dal quale non sarà più possibile aspettarsi una ripresa? Anche una profonda e duratura conoscenza tra paziente e medico non giova all'arte della prognosi; sembra addirittura che questa consuetudine tenda a rendere più ottimistica la prognosi del paziente. ${ }^{14-16}$ Un'ulteriore motivazione che rende difficile definire la terminalità, e perciò lo spostamento del focus dalla malattia al paziente privilegiando l'approccio palliativo, sono le significative deviazioni alle note traiettorie di malattia. Tali deviazioni hanno diverse determinanti su cui è necessario riflettere e soffermarsi: i) la variabilità individuale nella risposta o meno alle terapie e alle varie complicanze; ii) le esacerbazioni, spesso imprevedibili 
legate alle comorbilità del singolo paziente; iii) il sopraggiungere di nuove opportunità terapeutiche; iv) la sovrapposizione con la fisiologica terminalità dell'invecchiamento.

Conseguenza inevitabile della difficoltà ad identificare la fase terminale dell'IC è il rallentamento dell'attivazione delle cure palliative e della sospensione di trattamenti invasivi o aggressivi.

Peraltro, non vi è ormai dubbio che il trattamento palliativo anche nello specifico contesto dello scompenso cardiaco possa essere affiancato a quello attivo ed allora, a seconda della traiettoria di malattia, può essere difficile determinare l'enfasi da dare all'uno o all'altro nelle diverse fasi.

Ma anche quando sia definito il tempo delle cure palliative nell'insufficienza cardiaca end stage, resta da definire quali esse siano e come fornirle al paziente anche relativamente alla scelta del setting più adeguato. ${ }^{14,17-19}$

\section{Definizione dell'insufficienza cardiaca end stage}

La prevalenza dell'IC in Europa è stimata tra 0,4\%$2 \%$ con incremento lineare all'avanzare dell'età. Il $50 \%$ dei pazienti con IC muore entro 5 anni dalla formulazione della diagnosi mentre il $50 \%$ dei soggetti con fase avanzata di malattia (classe NYHA III-IV) muore entro 1 anno. L'età avanzata dei pazienti (76 anni in media) e l'elevata incidenza di comorbilità costituiscono importanti ragioni di tale infausta prognosi. ${ }^{20-24}$

Approssimativamente il 5\% dei pazienti con IC presentano malattia in fase end stage refrattaria alle terapie mediche. Rispetto a tali pazienti gli strumenti prognostici risultano di difficile utilità rispetto al singolo paziente e dai dati del SUPPORT Project si evince una particolare difficoltà prognostica dei curanti se è vero che meno del $75 \%$ dei pazienti arruolati è sopravvissuto più di sei mesi. Gli stessi interessanti dati mostrano che circa un quarto dei pazienti ospedalizzati per severo scompenso cardiaco aveva espresso la preferenza di non essere rianimato (in correlazione con età avanzata, percezione di una prognosi severa, compromissione dello stato funzionale e maggiore durata del ricovero) e che la percezione del medico rispetto alla preferenza del paziente non era accurata in circa un quarto dei casi; il miglioramento degli aspetti comunicativi mediante discussione approfondita ed esplicita delle tematiche del fine vita ed eventuali preferenze del paziente non si associava al raggiungimento di un maggior grado di concordanza tra paziente e medico ed a sostanziale modifica delle preferenze del paziente. ${ }^{25}$

Al tema dell'insufficienza cardiaca end stage le società scientifiche di area cardiologica hanno negli ul- timi hanno dedicato attenzione e specifici pronunciamenti ufficiali. Le Linee guida ESC $2016^{26}$ hanno identificato i pazienti affetti da insufficienza cardiaca da considerare end stage e nei quali quindi definire un approccio terapeutico palliativo focalizzato sui sintomi più che sulla progressione di malattia.

Le caratteristiche del paziente end-stage sono individuate in: i) un progressivo declino, fisico e mentale tale da configurare la dipendenza del paziente per la maggior parte delle attività quotidiane; ii) grave sintomatologia con scarsa qualità di vita; iii) frequenti ricoveri ospedalieri o episodi di scompenso nonostante ottimizzazione della terapia, esclusione della possibilità di trapianto o supporto meccanico; iv) cachessia cardiaca; v) giudizio clinico di fine vita nonostante l'ottimizzazione della terapia farmacologiche e non farmacologiche.

Un bel contributo alla definizione dell'insufficienza cardiaca end stage proviene anche da Linee guida di area nursing. ${ }^{27}$ Vengono in esse definiti gli indicatori clinici di declino specificamente connessi allo scompenso cardiaco $^{28}$ (Figura 2).

In generale gli indicatori generali di peggior prognosi (aspettativa di vita di poche settimane) includono uno stato di scarsa performance, la compromissione dello stato nutrizionale e un basso livello di albumina.

Tra gli strumenti prognostici raccomandati dalla Linea Guida vi è la Palliative Performance Scale ${ }^{29}$ (Tabella 1) che è anche lo strumento prescelto per la valutazione del paziente end stage in un documento SIAARTI del 2013. ${ }^{30}$

Tale documento, sforzo collaborativo tra diverse figure coinvolte nella gestione delle patologie end stage, è stato elaborato con l'obiettivo di identificare i criteri clinici e di valutazione globale disponibili in letteratura utili ad individuare i pazienti con insufficienza cronica end stage ed a consentire scelte appropriate di cura nonché a descrivere i criteri etici che ispirano le scelte terapeutiche e fornire un supporto agli operatori per coinvolgere i familiari nelle stesse.

La metodologia di fondo del documento è basata sulla valorizzazione di tutte le professionalità coinvolte nella gestione clinica dei pazienti nonché sulla massima considerazione per l'autodeterminazione del malato, sostenuto dalla sua rete di prossimità.

Sulla base di tali presupposti sono individuati i criteri clinici generali per inquadrare il paziente potenzialmente end stage (Tabella 2).

Sul tema specifico della insufficienza cardiaca è possibile definire tre fasi.

\section{I fase - Fase di gestione della patologia (NYHA I-II)}

Tale fase origina dal processo diagnostico e dalla impostazione terapeutica di base ed il paziente, informato compiutamente della diagnosi, delle prospettive 


\begin{tabular}{|c|c|}
\hline $\begin{array}{l}\text { Cardiopatia } \\
\text { congestizia }^{3}\end{array}$ & $\begin{array}{l}\text { Aspettative di vita }<6 \text { mesi: } \\
\text { - Dolore toracico, dispnea a riposo o con minimo sforzo, già } \\
\text { trattata in modo ottimale con diuretici e vasodilatatori. } \\
\text { - Insufficienza cardiaca congestizia }>2 \text { ricoveri nel corso } \\
\text { dell'anno. } \\
\text { - Aumento del } 50 \% \text { della dose di farmaco per via orale o aggiunta } \\
\text { di farmaci di nuova classe. } \\
\text { - Frazione di eiezione ventricolare sinistra }<20 \% \text {. } \\
\text { - Creatinina }>350 \mathrm{mmol} / \mathrm{L} \text {. }\end{array}$ \\
\hline & $\begin{array}{l}\text { Solo poche settimane: } \\
\text { - Anamnesi d'arresto cardiaco e di rianimazione } \\
\text { cardiopolmonare. } \\
\text { - Anamnesi di sincope inspiegata. } \\
\text { - Aritmie resistenti. } \\
\text { - Ipertensione. } \\
\text { - Diabete insulino-dipendente. } \\
\text { - Uso della nicotina. } \\
\text { - Precedente by-pass coronarico. }\end{array}$ \\
\hline
\end{tabular}

Figura 2. Indicatori specifici di declino dell'insufficienza cardiaca. Da: Registered Nurses'Association of Ontario. (2011). End-of-life Care During the Last Days and Hours. Toronto, ON.

Tabella 1. Scala funzionale per la valutazione globale e prognostica del malato (Palliative Performance Scale).

\begin{tabular}{|c|c|c|c|c|c|}
\hline$\%$ & Deambulazione & $\begin{array}{l}\text { Livello di attività } \\
\text { Evidenza di malattia }\end{array}$ & Cura di sé & $\begin{array}{l}\text { Introduzione liquidi } \\
\text { e/o solidi }\end{array}$ & Livello di coscienza \\
\hline 100 & Normale & $\begin{array}{l}\text { Normale } \\
\text { Nessuna malattia }\end{array}$ & Completamente autonoma & Normale & Normale \\
\hline 90 & Normale & $\begin{array}{l}\text { Normale } \\
\text { Qualche grado di malattia }\end{array}$ & Completamente autonoma & Normale & Normale \\
\hline 80 & Normale & $\begin{array}{l}\text { Normale con sforzo } \\
\text { Qualche grado di malattia }\end{array}$ & Completamente autonoma & Normale o ridotta & Normale \\
\hline 70 & Ridotta & $\begin{array}{l}\text { Non può svolgere un } \\
\text { compito o lavoro } \\
\text { Qualche grado di malattia }\end{array}$ & Completamente autonoma & Come sopra & Normale \\
\hline 60 & Ridotta & $\begin{array}{l}\text { Non può svolgere hobbies } \\
\text { o lavori di casa } \\
\text { Malattia significativa }\end{array}$ & $\begin{array}{l}\text { Assistenza occasionale } \\
\text { Assistenza richiesta }\end{array}$ & Come sopra & Normale o confuso/a \\
\hline 50 & $\begin{array}{l}\text { Prevalentemente } \\
\text { seduto/a o disteso/a }\end{array}$ & $\begin{array}{l}\text { Non può svolgere alcun lavoro } \\
\text { Malattia estesa }\end{array}$ & $\begin{array}{l}\text { Assistenza considerevole } \\
\text { Assistenza richiesta }\end{array}$ & Come sopra & Normale o confuso/a \\
\hline 40 & $\begin{array}{l}\text { Prevalentemente } \\
\text { a letto }\end{array}$ & Come sopra & Prevalentemente assistito & Come sopra & $\begin{array}{l}\text { Normale o sonnolento/a } \\
\text { o confuso/a }\end{array}$ \\
\hline 30 & Allettato/a & Come sopra & Assistenza totale & Ridotta & Come sopra \\
\hline 20 & Allettato/a & Come sopra & Come sopra & Minima & Come sopra \\
\hline 10 & Allettato/a & Come sopra & Come sopra & Solo cura della bocca & Sonnolento/a o in coma \\
\hline 0 & Morte & - & - & - & - \\
\hline
\end{tabular}


terapeutiche attuali e della possibile prognosi, può sin dall'inizio contribuire ad una pianificazione anticipata e condivisa del percorso di cura.

\section{II fase (NYHA III-IV) - Fase di supporto e palliativa}

Tale fase di caratterizza per le ripetute ospedalizzazioni e necessità di terapie e supporti avanzati non disgiunte dal controllo dei sintomi e dalle considerazioni sulla qualità di vita del paziente con approccio multidisciplinare ed olistico. In questa seconda fase, congiuntamente al paziente ed ai suoi familiari, è opportuno ridiscutere le scelte terapeutiche ed il piano di cura.

\section{III fase (NYHA IV) - Fase terminale propriamente detta}

Tale fase è dominata da disfunzione renale, ipotensione, edemi refrattari, astenia, dispnea e cachessia.

L'obiettivo terapeutico di questa fase è incentrato sul controllo dei sintomi e valutazione dei bisogni globali con ampia discussione su cure di fine vita ed individuazione dell'ambiente più idoneo ad erogarle (coinvolgendo anche i familiari).

Ai criteri clinici generali e specifici per patologia si propone di affiancare alcuni elementi valutativi di ordine generale in un approccio più squisitamente palliativistico (Tabella 3).

Sulla base di tutte le precedenti considerazioni, può essere considerato end stage il paziente con insufficienza cardiaca cronica che, nonostante terapia ottimale, presenti (Tabelle 4-6): i) classe NYHA IV; ii) ipotensione arteriosa e/o ritenzione di liquidi; iii) segni di cachessia; iv) più di una ospedalizzazione negli ultimi 6 mesi; v) scarsa risposta alla resincronizzazione cardiaca quando indicata; vi) necessità di frequente $\mathrm{o}$ continuo supporto farmacologico infusionale.

\section{Ruolo della terapia palliativa}

Le componenti chiave delle cure palliative nel contesto dello scompenso cardiaco, a seconda della fase in cui si sia definito di introdurle, sono da ricercare in primis nel focus sul miglioramento della qualità di vita del paziente (e dei suoi familiari), quindi nella frequente valutazione dei sintomi (compresa dispnea e dolore) legati all'avanzamento dello stato di malattia ed alle comorbilità focalizzandosi sul controllo degli stessi; nel garantire al paziente ed ai familiari supporto psicologico e spirituale secondo necessità ed infine nel definire un programma avanzato di terapia comprendente anche preferenze su luogo della morte, eventuale rianimazione (e disattivazione di device quali pace maker e defibrillatori). ${ }^{28,31-33}$

\section{Dispositivi impiantabili ed insufficienza cardiaca end-stage: un approccio palliativo}

Poiché il numero di pazienti affetti da insufficienza cardiaca trattati con impianto di pacemaker (PM) e defibrillatori (ICD) con eventuale supporto biventricolare (di fatto terapie di supporto a funzioni vitali compromesse) è in progressivo aumento, la problematica concernente la loro disattivazione nei pazienti inquadrati come end stage si pone e si porrà sempre più frequentemente; di ciò esiste infatti specifica menzione anche nelle posizioni delle Linee guida sia di area cardiologica che palliativistica. ${ }^{33-38}$

Il tema è indubbiamente complesso anche per la

Tabella 2. Criteri clinici generali per inquadrare il paziente potenzialmente end-stage.

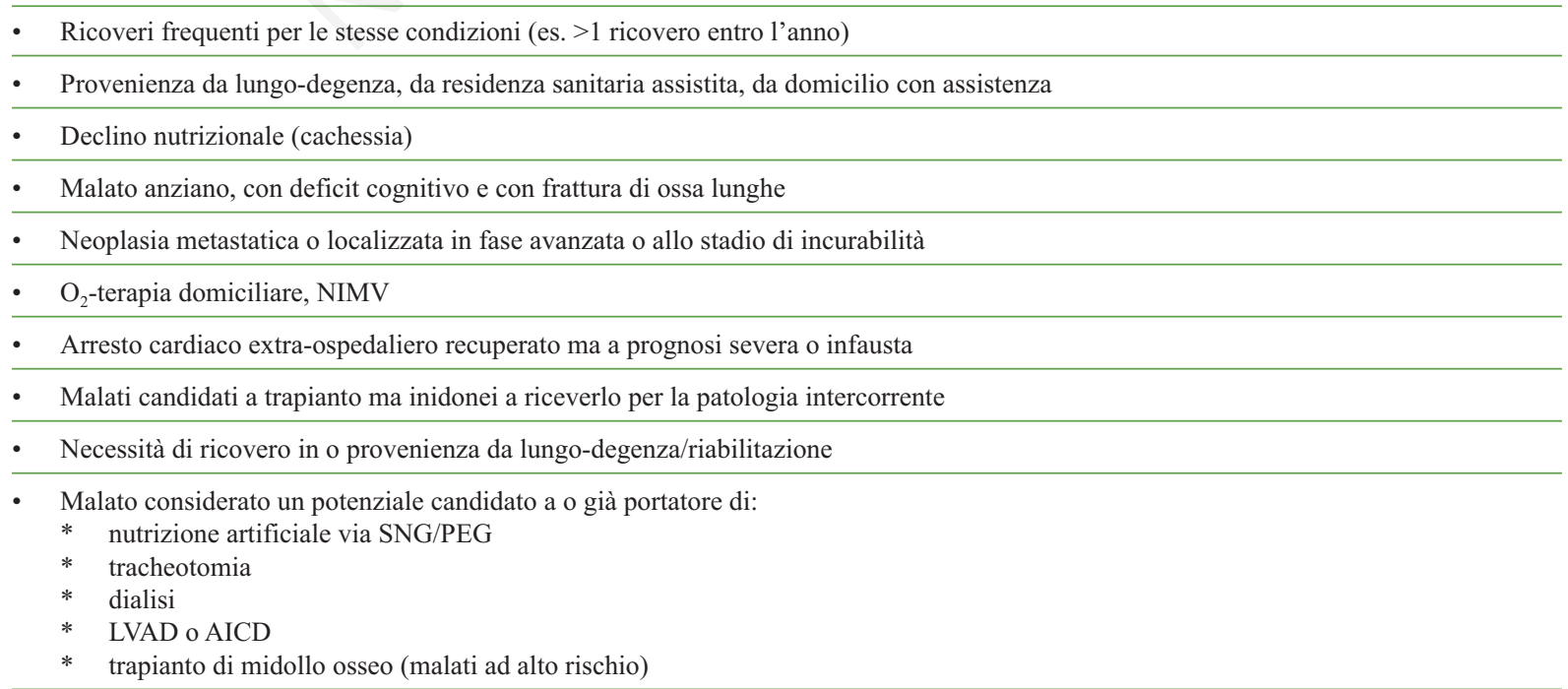

Da: SIAARTI Grandi insufficienze d'organo end stage: cure intensive o palliative? (2013). 
Tabella 3. Quesiti integrativi dei criteri clinici specifici e generali.

\begin{tabular}{|c|c|}
\hline Sintomo di valutazione & $\begin{array}{l}\text { - } \quad \text { Ci sono sintomi fisici o psicologici rilevanti e angoscianti? } \\
\text { Il ricovero viene richiesto per difficile controllo di sintomi cronici (sintomo di moderata-grave } \\
\text { intensità durata }>24-48 \text { h)? }\end{array}$ \\
\hline Valutazione sociale & - $\quad$ Ci sono significative problematiche sociali? \\
\hline Valutazione dei bisogni spirituali & - $\quad$ Ci sono significativi bisogni spirituali? \\
\hline $\begin{array}{l}\text { Identificazione degli obiettivi } \\
\text { di cura centrati sul malato }\end{array}$ & $\begin{array}{l}\text { - Vi è chiarezza e documentazione sugli obiettivi di cura? } \\
\text { - Vi è chiarezza e documentazione sugli obiettivi di cura? } \\
\text { - Vi sono incertezze o disaccordi tra malato, personale e/o familiari in materia di: } \\
\text { * Decisioni relative a trattamenti medici aggressivi? } \\
\text { * Preferenze sui trattamenti intensivi? } \\
\text { - Quali sono le aspettative di approfondimento diagnostico, di cura e assistenza identificate dal } \\
\text { malato, dalla famiglia, dal legittimo rappresentante? } \\
\text { - Le opzioni di trattamento sono in linea con le aspettative di cura identificate dal malato, dalla } \\
\text { - Samiglia, dal legittimo rappresentante? } \\
\text { - Sono state esplicitate dal malato particolari scelte etiche (rifiuto/accettazione di determinati tipi di } \\
\text { approfondimento diagnostico e di trattamento rispetto ad altri)? } \\
\text { - Il malato ha già partecipato ad una pianificazione anticipata della cura? }\end{array}$ \\
\hline $\begin{array}{l}\text { Comprensione delle opzioni } \\
\text { di prognosi/trattamento }\end{array}$ & $\begin{array}{l}\text { - Il malato e la famiglia comprendono e condividono le scelte relative alla fase attuale della traiettoria } \\
\text { di malattia, alla prognosi, e alle opzioni di trattamento? } \\
\text { - Se la durata stimata dell'eventuale ricovero in TI è } \geq 7 \text { gg il malato e la famiglia lo accettano? }\end{array}$ \\
\hline Invio a cure post-dimissione & $\begin{array}{l}\text { - Quali sono i fattori chiave per un passaggio sicuro da un ambiente ad un altro? } \\
\text { - E stato contattato il mmg dopo il ricovero? } \\
\text { - La dimissione è stata concordata con il Mmg? }\end{array}$ \\
\hline
\end{tabular}

Da: SIAARTI Grandi insufficienze d'organo end stage: cure intensive o palliative? (2013).

Tabella 4. Criteri specifici per la definizione di scompenso cardiaco end stage.

\begin{tabular}{ll}
\hline- & Classe NYHA IV \\
\hline- & $>1$ ospedalizzazione negli ultimi 6 mesi \\
\hline - & Ipotensione periferica e/o ritenzione di liquidi \\
\hline - & Necessità di frequente o continuo supporto farmacologico infusionale \\
\hline & Scarsa risposta alla resincronizzazione cardiaca quando indicata \\
\hline
\end{tabular}

Tabella 5. Tipologia di pazienti con insufficienza cardiaca in cui è indicato prendere in considerazione cure di fine vita.

- Progressivo declino funzionale (fisico e mentale) e dipendenza nella maggior parte delle attività quotidiane

- Severa sintomatologia con povera qualità di vita nonostante l'ottimizzazione delle terapie farmacologiche e non farmacologiche

- Esclusione della possibilità di trapianto cardiaco e supporto circolatorio meccanico

Tabella 6. Componenti "chiave" delle cure palliative nei pazienti con scompenso cardiaco.

- Miglioramento e mantenimento nelle migliori condizioni possibili della qualità di vita del paziente e della sua famiglia

- Frequente valutazione dei sintomi (soprattutto dispnea e dolore) riferibili allo scompenso cardiaco ed alle altre comorbilità e trattamento mirato per la risoluzione degli stessi

- Definire un piano di cura che tenga conto delle preferenze del paziente anche in ordine alla disattivazione di eventuali device e scelta del luogo della morte 
mancata conoscenza da parte dei pazienti e la scarsa confidenza dei medici con gli strumenti culturali per discuterne con pazienti e familiari; un modo per affrontarlo è operare una distinzione fra la scelta della disattivazione di un PM (soprattutto nei malati PMdipendenti) e di ICD. ${ }^{33,36}$

In quest'ultimo caso, infatti, il quesito riguarda la necessità di risparmiare al paziente in fase terminale gli interventi del dispositivo quando non siano in grado di apportare un sostanziale miglioramento della durata e della qualità della vita residua. Si stima che il $20 \%$ dei pazienti portatori di ICD manifestino uno shock durante l'ultima settimana di vita sperimentando quindi dolore e peggioramento della qualità della vita residua.

Sul piano etico quindi, la disattivazione di un ICD è un'opzione possibile (così come il rifiuto e l'interruzione di una terapia medica) e coerente con l'approccio palliativo di fine vita. Essa tuttavia, come raccomandato da documenti di consenso disponibili, deve essere il punto finale di un processo trasparente e deliberato, con piena e documentata tracciabilità della decisione da parte del paziente e del medico. Peraltro, la possibilità di disattivare un ICD, in caso di peggioramento delle condizioni di salute, dovrebbe essere discussa con il paziente e la sua famiglia al momento dell'impianto e far parte integrante del consenso informato. È ovviamente raccomandato che al paziente che abbia scelto di disattivare un ICD debba essere garantito che un eventuale ripensamento sarà accolto e il dispositivo riattivato.

Più controversa è invece la scelta concernente la disattivazione del PM, nei malati PM-dipendenti, poiché la stessa provocherebbe la morte immediata ponendo il dubbio etico del configurarsi la condizione di eutanasia o suicidio assistito. Nel caso di pazienti non PM-dipendenti e in quelli sottoposti a stimolazione biventricolare, la disattivazione non provoca la morte immediata o in tempi rapidi, ma potrebbe invece peggiorare la qualità della vita del malato (inducendo bradicardia sintomatica o deterioramento emodinamico) e ciò sarebbe in contrasto con la finalità dell'approccio palliativo. Invece, in caso di imminente esaurimento del generatore può essere opportuno valutare di non procedere alla sua sostituzione.

Un tema affine è quello relativo alla disattivazione dei dispositivi per l'assistenza al circolo Left Ventricular Assist Devices (LVAD) il cui impiego, anche come Destination Therapy (DT) nei pazienti con scompenso refrattario ed avanzato non candidabili al trapianto, è in incremento (un centinaio di malati/anno in Italia). La sopravvivenza a 2 anni di pazienti sottoposti ad impianto per DT è del $50 \%$ circa e la morte può sopraggiungere per progressione dell'insufficienza cardiaca o per complicanze relate al LVAD..$^{38-40}$

È stato recentemente proposto che la disattivazione di un LVAD possa essere considerata eticamente possibile, come approccio palliativo, in tutte le condizioni cliniche estreme (coma irreversibile, shock, infezioni, insufficienza multi organo, default del dispositivo) e non solo nell'end stage della insufficienza cardiaca. ${ }^{40}$

\section{Opzioni terapeutiche specifiche e setting di cura}

I pazienti con insufficienza cardiaca end stage manifestano una importante quota di sintomi comprendenti dolore, dispnea, astenia, depressione, edema, insonnia, ansia, anoressia e confusione che nell'insieme delineano una qualità di vita estremamente scarsa e che sono comparabili, se non superiori, a quelli sperimentati dai pazienti neoplastici. ${ }^{41,42}$ In questo ambito le terapia palliative hanno dimostrato di migliorare gli outcomes centrati sulla soddisfazione dei pazienti in una varietà di popolazioni nonché di minimizzare le ospedalizzazioni e ridurre i costi globali dell'insufficienza cardiaca. ${ }^{43-52}$

Le terapie specifiche per il controllo dei sintomi, anche se con basso livello di evidenza, sono costituite essenzialmente dall'utilizzo di morfina, ${ }^{51}$ incremento della concentrazione di ossigeno, utilizzo dei diuretici per ridurre la congestione la dispnea, riduzione della dose di antipertensivi per minimizzare il rischio di cadute. ${ }^{26,52-54}$

Tutte queste terapie, idealmente e possibilmente, dovrebbero essere effettuate a domicilio del paziente fornendo alla famiglia tutto il necessario supporto.

La scelta del luogo di cura nella fase finale di malattia è cruciale e dovrebbe essere affrontata e condivisa anticipatamente con il paziente e la sua famiglia anche perché la stessa influenza obbligatoriamente le scelte di trattamento.

Qualora il ricovero ospedaliero sia la scelta preferenziale, andrà privilegiata la collocazione del paziente in degenza ordinaria (minori vincoli di monitoraggio ed auspicabilmente maggiore disponibilità alla presenza dei familiari piuttosto che in ambiente intensivo. ${ }^{30}$

Le tematiche in discussione dovrebbero trovare posto in una adeguata pianificazione delle cure e delle cure di fine vita, attraverso direttive anticipate; ciò potrebbe effettivamente chiarire le preferenze del paziente posto che siano sempre esplicitate allo stesso ed ai familiari tutte le informazioni necessarie per esprimerle.

\section{Conclusioni}

La progressione dell'insufficienza cardiaca, nonostante lo sviluppo di terapie farmacologiche e non farmacologiche sempre più avanzate, costituisce una 
condizione di particolare disagio per i pazienti (ed i loro familiari) con importanti sequele fisiche e psicologiche (tra cui depressione e difficoltà psicosociali). L'elevata mortalità e la scarsa qualità di vita dei pazienti hanno portato a ripensare la strategia di gestione globale dell'insufficienza cardiaca sviluppando un approccio multidisciplinare volto a migliorare i sintomi ed il dolore in tutte le fasi del continuum fisiopatologico dello scompenso (anche in associazione alla migliore terapia usuale per ciascuna fase) ma soprattutto nel contesto dell'end stage. Tale approccio, che ha nella comunicazione, nel supporto emozionale, nella condivisione delle scelte di cura e nella definizione anticipata di un piano di trattamento gli elementi caratterizzanti, va introdotto precocemente nella traiettoria di malattia con potenziamento corrispondente alla progressione della stessa ed ha dimostrato di essere in grado di migliorare la qualità di vita dei pazienti con insufficienza cardiaca. ${ }^{43,45,53-58}$

\section{Bibliografia}

1. Il paziente terminale, Il Caleidoscopio italiano, Ario Zilli, Genova 1987

2. Turriziani A, Terapie di supporto, cure palliative e oncologia: un contributo alla chiarezza. RICP 2012;14,2:5-84.

3. Gambacorta M, Campanini M, Nardi R, Le problematiche di fine vita: quale consapevolezza da parte degli internisti? Quaderni -Ital J Med 2017; 5(4):5-22

4. Lusiani L, Bullo C, Il concetto di terminalità: certezze e incertezze. Quaderni - Ital J Med 2017; 5(4):23-27

5. Http://www.gmc-uk.org/static/documents/content/End of_life.pdf (last accessed January 2013)

6. http://publications.nice.org.uk/quality-standard-for-endof-life-care-for-adults-qs 13/quality-statement-3- assessment-care-planning-and-review (last accessed January 2013)

7. AHRQ Agency for Healthcare Research and Quality Dec. 2004 "End-of-life care and outcomes"

8. The'Surprise question': Lynn J 2005 Altarum Institute Center for Elder Care and Advanced Illness www.thehastingscenter.org/pdf/living-long-in-fragile-health.pdf

9. Thomas K et al. Prognostic indicator Guidance 4 Edition.

10. Chesi G, Montanari P, Nardi R. Cure palliative: nuova branca specialistica o competenze da riscoprire? Quaderni Ital J Med 2015;3:583-7.

11. D'Amore F, The doctor and the patient: doing too much or too little. Ital J Med 2013;7:135-7.

12. Gawande A, What should medicine do when it can't save you? Annals of Medicine, August 22010

13. Murtagh FEM, Preston M. Higginson I. Patterns of dying :palliative care for non malignant disease. Clin Med 2004, 4 39-44

14. Lunney JR, Lynn J, Foley DJ et al, Pattern of functional decline at the end of life. JAMA 2003; 289(18):2387-92

15. Christakis N, Lamont E, Extent and determinant of error in doctors'prognoses in terminally ill patients: prospective cohort study. BMJ 2000; 320(7233):469-73
16. Magnani L, Traiettorie di malattia: non sempre i pazienti e le famiglie sono informati. Quaderni- Ital J Med 2017; 5(4):28-3143.

17. Ruegger J, Hodgkinson S, Field-Smith S, Ahmedzai SH, Care of adults in the last days of life: summary of NICE guidance. BMJ 2015;305:h6631 doi:10.1136/bmmj. h6631.

18. Kelley AS, Treatment intensity at end of life - time to act on the evidence. Lancet 2011;378:1364-5

19. Roger VL, Weston SA, Redfield MM et al. Trends in heart failure incidence and survival in a communitybased population. JAMA. 2004;292:344 -350.

20. Hunt SA, Baker DW, Chin MH et al. ACC/AHA guidelines for the evaluation and management of chronic heart failure in the adult: executive summary: a report of the American College of Cardiology/ American Heart Association Task Force on Practice Guidelines (Committee to revise the 1995 Guidelines for the Evaluation and Management of Heart Failure). J Am Coll Cardiol 2001;38:2101-13.

21. McMurray JJV, Adamopoulos S, Anker SD et al: ESC Guidelines for the diagnosis and treatment of acute and chronic heart failure 2012. The Task Force for the diagnosis and Treatment of Acute and Chronic Heart Failure 2012 of the European Society of Cardiology. Developed in collaboration with the Heart Failure Association (HFA) of the ESC. Eur Heart J 2012:33;1787-1847

22. Lindenfeld J, Albert NM, Boehmer JP et al: HFSA 2010 Comprehensive Heart Failure Practice Guidelines. J Card Fail. 2010; 16:1-194

23. Wong CY, Chaudhry SI, Desai MM, Krumholz HM, Trends in comorbidity, disability, and polypharmacy in heart failure. Am J Med 2011;124:136-143

24. CostanzoMR, Mills RM,Wynne J, Characteristics of "StageD" heart failure: Insights from the Acute Decompensated

25. Krumholz H, Phillips R, Hammel MB, Teno J et al. Resuscitation preferences among patients with severe congestive heart failure. Results from the SUPPORT project. Circulation. 1998;98:648-655

26. Ponikowski P, Voors A.A, Anker S.D et al 2016 ESC Guidelines for diagnosis and treatment of acute and chronic heart failure, European Journal of Heart Failure ( 2016) doi:10.1002/ejhf.592

27. Registered Nurses' Association of Ontario. (2011). Endof-life Care During the Last Days and Hours. Toronto, ON: Registered Nurses' Association of Ontario. Clinical Best Practice Guidelines

28. Derfler M, Jacob M, Wolf RE, Bleyer F et al. Mode of death from congestive heart failure: implications for clinical management. Am J Geriatr Cardiol. 2004; 13(6): 299-304.29.

29. Anderson, F., Downing, G. M., Hill et al. . (1996). Palliative Performance Scale (PPS): A new tool. Journal of Palliative Care, 12(1), 5-11.

30. SIAARTI Grandi insufficienze d'organo end stage: cure intensive o cure palliative? Documento condiviso per una pianificazione delle scelte di cura (2013)

31. Romanò M, Graziano G, Zucco F, Le cure palliative nel trattamento della insufficienza cardiaca avanzata. Rivista Italiana di Cure Palliative. 2006;4:37-44

32. Jaarsma T, Beattie JM, Ryder M et al. Palliative care in heart failure: a position statement from the palliative 
care workshop of the Heart Failure Association of the European Heart Society. Eur J Heart Fail. 2009; 11: 433-443

33. Waterhouse E, Ahmad F, Do implantable cardioverter defibrillators complicate end-of-life care for those with heart failure? Curr Opin Support Palliat Care. 2011; 5:307-311

34. Mc Geary A, Eldergill S, Medicolegal issues arising when pacemaker and implantable cardioverter defibrillator devices are deactivated in terminally ill patients. Med Sci Law. 2010,50:40-44

35. Padeletti L, Arnar DO, Boncinelli L et al. EHRA (European Heart Rhythm Association) Expert Consensus Statement on the management of cardiovascular implantable electronic devices in patients nearing end of life or requesting withdrawal of therapy. Europace. 2010;12:1480-1489

36. Morgenweck CJ, Ethical considerations for discontinuing pacemakers and automatic implantable cardiac defibrillators at the end-of-life. Curr Opin Anaesthesiol. 2013; 26:171-175

37. Kramer DB, Kesselheim AS, Brock DW et al. Ethical and legal views of physicians regarding deactivation of cardiac implantable electrical devices: A quantitative assessment. Heart Rhythm. 2010;7:1537-1542

38. Kirklin JK, Naftel DC, Kormos RL et al. Third INTERMACS Annual report: the evolution of destination therapy in the United States. J Heart Lung Transplant. 2011;30:115-123

39. Martinelli L, Cannata A, Oliva F, Frigerio M, Nuove strategie per il trattamento dell'insufficienza cardiaca refrattaria: Chi avviare al supporto meccanico "longterm"? Cardiologia 2012. Atti del 46 Corso Dipartimento Cardiotoracovascolare "A. DE GASPERIS" pag. 437-440

40. Rady M, Verheijde JL, Ethical Challenges With Deactivation of Durable Mechanical Circulatory Support at the End of Life: Left Ventricular Assist Devices and Total Artificial Hearts. J Intensive Care Med published online 6 March 2012. DOI: 10.1177/0885066611432415

41. Bekelman DB, Havranek EP, Becker DM, et al.: Symptoms, depression, and quality of life in patients with heart failure. J Card Fail 2007;13:643-648.

42. Evangelista LS, Lombardo D, Malik S et al . Examining the effects of an outpatient palliative care consultation on symptom burden, depression, and quality of life in patients with symptomatic heart failure. J Card Fail 2012;18:894-899

43. Brannstrom M, Boman K, Effects of person-centred and integrated chronic heart failure and palliative home care. PREFER: a randomized controlled study. Eur J Heart Fail 2014;16:1142-1151.

44. Diop MSA, Rudolph JL, Zimmermann KM et al. Pal- liative Care Interventions for Patients with Heart Failure:A Systematic Review and Meta-Analysis . Journal of Palliative Medicine Volume 20, Number 1, 2017

45. Rogers JG, Patel CB, Mentz RJ et al. The Palliative Care in Heart Failure (PAL-HF) Randomized, Controlled Clinical. J Am Coll Cardiol. 2017 July 18; 70(3):

46. Shah AB, Morrissey RP, Baraghoush A, et al.: Failing the failing heart: A review of palliative care in heart failure.Rev Cardiovasc Med 2013;14:41-48.

47. Goodlin SJ: Palliative care for end-stage heart failure. Curr Heart Fail Rep 2005;2:155-160.

48. McCarthy M, Lay M, Addington-Hall J: Dying from heart disease. J R Coll Physicians Lond 1996;30:325-328.

49. Lynn J, Teno JM, Phillips RS, et al.: Perceptions by family members of the dying experience of older and seriously ill patients. SUPPORT Investigators. Study to understand prognoses and preferences for outcomes and risks of treatments. Ann Intern Med 1997;126:97-106.

50. Pattenden JF, Mason AR, Lewin RJ: Collaborative palliative care for advanced heart failure: Outcomes and costs from the 'Better Together' pilot study. BMJ Support Palliat Care 2013;3:69-76.

51. Johnson MJ, McDonagh TA, Harkness A, et al. Morphine for the relief of breathlessness in patients with chronic heart failure - a pilot study. Eur J Heart Fail 2002;4:753-756.

52. Jaarsma T, Beattie JM, Ryder M et al . Palliative care in heart failure: a position statement from the palliative care workshop of the Heart Failure Association of the European Society of Cardiology. Eur J Heart Fail 2009; 11:433-443.

53. McIlvennan CK, Allen LA, Palliative care in patients with heart failure BMJ 2016;353:i1010

54. Denvir MA, Murray SA, Boyd KJ, Future care planning: a first step to palliative care for all patients with advanced heart disease. Heart 2015;101:1002-1007.

55. Dev S, Abernethy AP, Rogers JG et al. Preferences of people with advanced heart failure-A structured narrative literature review to inform decision making in the palliative care setting. Am Heart J 2012;164:313319.e5.

56. Schellinger S, Sidebottom A, Briggs L: Disease specific advance care planning for heart failure patients: Implementation in a large health system. J Palliat Med 2011;14:1224-1230.

57. Parry R, Land V, Seymour J, How to communicate with patients about future illness progression and end of life: a systematic review. BMJ Supportive \& Palliative Care 2014;4:331-41.

58. Levenson JW, McCarthy EP, Lynn J, et al.: The last six months of life for patients with congestive heart failure. JAm Geriatr Soc 2000;48(5 Suppl):S101-S10 


\section{LINEE GUIDA PER GLI AUTORI}

I Quaderni dell 'Italian Journal of Medicine (Quaderni ITJM), costituiscono una collana supplementare solo online annessa alla rivista Italian Journal of Medicine contenente lavori solo in lingua italiana .

I Quaderni ITJM pubblicano:

- Monografie ad hoc individuate dal Presidente FADOI, dal Consiglio Direttivo, dal Board Scientifico o dall'Editor in Chief dell'Italian Journal of Medicine, in funzione del contesto scientifico-istituzionale attuale.

- Monografie ad hoc su temi di particolare rilevanza scientifica a cura della Commissione FADOI Giovani.

- Traduzioni in italiano di alcuni lavori pubblicati sui numeri standard dell'Italian Journal of Medicine, di particolare interesse per la comunità scientifica.

\section{STESURA DEI LAVORI}

I lavori dovranno essere redatti in modo conforme alle linee guida sotto riportate:

- I manoscritti devono essere scritti interamente in lingua italiana, su documento di Word, con carattere Times New Roman/Arial, dimensione 12, formato A4, interlinea doppia e margini $2,54 \mathrm{~cm}$

Parole totali: $\max$ 4000; Sommario/Abstract: $\max 250$ parole; Bibliografia: min 40 voci; Tabelle e Figure: $3 / 5$ totali (le tabelle non devono superare n. 1 pagina del documento in Word).

- La strutturazione del contenuto deve attenersi agli standard internazionali per la Rassegna (Review): i) Abstract riassuntivo dell'intero lavoro; ii) Introduzione al tema trattato; iii) Criteri e strumenti di ricerca (criteri di inclusione/esclusione, banche dati consultate, ...); iv) i successivi paragrafi devono illustrare le più recenti scoperte scientifiche nel settore; v) Conclusioni; vi) Bibliografia.

- La prima pagina deve riportare: i) titolo (in stampatello minuscolo), senza acronimi; ii) nome e cognome per esteso di ciascun autore; iii) affiliazione(i) di ciascun autore, numerate con numeri arabi iv) eventuali ringraziamenti; v) nome e indirizzo postale completi dell'autore corrispondente, corredati da telefono, fax, e-mail; vi) da 3 a 5 parole chiave, separate da virgola. La seconda pagina può riportare: i) contributi degli autori, e.g. informazioni relative a contributi sostanziali delle persone coinvolte nello studio (http://www.icmje.org/\#author); ii) dichiarazione relativa a potenziali conflitti d'interesse; iii) ulteriori informazioni (e.g. fondi, esposizioni durante conferenze...).

- In caso di utilizzo di tabelle, queste devono essere tutte numerate con numeri arabi e citate nel testo in ordine consecutivo (e.g. NON nominare le tabelle come Tabella $1 \mathrm{~A}, 1 \mathrm{~B}, \ldots$ o $1.0,1.1, \ldots)$. Le tabelle devono essere presentate in formato editabile. Ciascuna tabella deve essere corredata da una breve didascalia; in caso di abbreviazioni, riportare una nota a piè di CIASCUNA tabella che spieghi TUTTE le abbreviazioni presenti in ognuna.

- In caso di utilizzo di figure, queste devono essere inviate in formato tiff o .jpg, allegate al manoscritto in singoli files, secondo le seguenti specifiche:

i) a colori (salvate in modalità CMYK): minimo 300 dpi di risoluzione;

ii) in bianco e nero: minimo $600 \mathrm{dpi}$ di risoluzione;

iii) minimo $17,5 \mathrm{~cm}$ di larghezza.

Ciascuna figura deve essere corredata da una breve didascalia.

$N B$ : In caso di Tabelle/Figure riprese e/o modificate da altri lavori già pubblicati, sarà cura degli autori accertarsi se tali materiali siano o meno coperti da copyright e procurarsi i permessi necessari per la riproduzione. Tali permessi dovranno essere allegati alla versione definitiva del lavoro. L'ufficio editoriale si riserva la facoltà di rimuovere Tabelle/Figure coperte da copyright, se sprovviste dei necessari permessi.

- In caso di utilizzo di abbreviazioni, la prima volta che esse sono citate è necessario scrivere per esteso la definizione+abbreviazione tra parentesi tonde [e.g. risonanza magnetica $(\mathrm{RMN})]$, a seguire si dovrà riportare solo l'abbreviazione (unica eccezione: nei titoli e nelle didascalie di tabelle e figure NON si utilizzano abbreviazioni).

\section{BIBLIOGRAFIA}

Le voci bibliografiche devono essere formattate secondo lo stile Vancouver.

Nella sezione Bibliografia, le voci bibliografiche devono essere numerate consecutivamente nell'ordine in cui appaiono per la prima volta nel testo (NON in ordine alfabetico) e, nel testo, devono essere indicate con numeri arabi in apice. Voci bibliografiche riferite a comunicazioni personali o dati non pubblicati devono essere incorporate nel testo e NON inserite tra le voci numerate [e.g. (Wright 2011, dati non pubblicati) o (Wright 2011, comunicazione personale)]. Le voci bibliografiche nella sezione Bibliografia devono tassativamente essere preparate come segue:

i) più di 3 autori, citare 3 autori, et al. Se il lavoro contiene solo 4 autori, citarli tutti e 4 ;

ii) titolo del lavoro in stampatello minuscolo;

iii) nome della rivista, senza punti, abbreviato secondo gli standard internazionali; in caso di dubbi sulla corretta abbreviazione, fare riferimento ai seguenti siti:

a. ISI Journal Abbreviations Index (http://library.caltech.edu/ reference/abbreviations/);

b. Biological Journals and Abbreviations (http://home.ncifcrf. gov/research/bja/);

c. Medline List of Journal Titles (ftp://ftp.ncbi.nih.gov/pubmed/J_Medline.txt);

iv) inserire l'anno di pubblicazione subito dopo il nome della rivista, seguito da punto e virgola;

v) NON inserire giorno o mese di pubblicazione;

vi) citare solo il volume, seguito dai due punti (NON citare il fascicolo tra parentesi);

vii) abbreviare le pagine, e.g. 351-8

Per accertarsi di aver correttamente formattato le voci bibliografiche, confrontarle con le citazioni in PubMed (http://www.ncbi. nlm.nih.gov/pubmed).

Esempi (prestare attenzione anche alla punteggiatura):

Articolo standard su Rivista

Halpern SD, Ubel PA, Caplan AL. Solid-organ transplantation in HIV-infected patients. N Engl J Med 2002;347:284-7.

Proceedings

Christensen S, Oppacher F. An analysis of Koza's computational effort statistic for genetic programming. In: Foster JA, Lutton E, Miller J, Ryan C, Tettamanzi AG, eds. Genetic programming. EuroGP 2002: Proceedings of the 5th European Conference on Genetic Programming, 2002 Apr 3-5, Kinsdale, Ireland. Berlin: Springer; 2002. pp 182-91.

Articoli i cui autori sono Organizzazioni

Diabetes Prevention Program Research Group. Hypertension, insulin, and proinsulin in participants with impaired glucose tolerance. Hypertension 2002;40:679-86

Libri

Murray PR, Rosenthal KS, Kobayashi GS, Pfaller MA. Medical microbiology. 4th ed. St. Louis, MO: Mosby; 2002. (CITAZIONE DEL LIBRO INTERO)

Meltzer PS, Kallioniemi A, Trent JM. Chromosome alterations in human solid tumors. In: Vogelstein B, Kinzler KW, eds. The genetic basis of human cancer. New York, NY: McGraw-Hill; 2002. pp 93113. (CITAZIONE DI UN CAPITOLO)

\section{MODALITÀ D'INVIO DEI LAVORI}

Monografie

Gli Autori dovranno fare riferimento all'Editor-in-Chief o alle persone da lui designate nelle lettere di invito a scrivere gli articoli programmati.

Per gli articoli inviati da giovani internisti, gli Autori dovranno fare riferimento al Dr. Michele Meschi (e-mail:mmeschi@ausl.pr.it)e agli eventuali altri referenti da lei designati, nelle prime fasi di stesura dei manoscritti, revisioni e correzioni. 


\title{
QUADERNI
}

\author{
dell'Italian Journal
}

of Medicine

Il Dr. Meschi raccoglierà poi le versioni definitive dei lavori di ciascuna monografia e provvederà all'invio di tutti i materiali all'ufficio editoriale.

I lavori solo nella loro versione definitiva e approvata dalla Commissione FADOI Giovani dovranno pervenire all'ufficio editoriale già pronti per l'impaginazione e immediata pubblicazione (già corredati da eventuali permessi per la riproduzione di tabelle e immagini redatti secondo le presenti linee guida).

\section{Traduzioni}

Previo invito dell'Editor-in-Chief, gli Autori dovranno far pervenire all'ufficio editoriale la versione tradotta in italiano, al seguente indirizzo e-mail: paola.granata@pagepress.org

Il file in formato Word dovrà essere formattato secondo gli standard editoriali della rivista ufficiale ed essere già pronto per impagina- zione e immediata pubblicazione (corredato da eventuali permessi per la riproduzione di tabelle e immagini).

Si prega di inviare le eventuali tabelle in formato editabile e le figure in alta definizione secondo gli standard sopra riportati.

\section{NOTA PER GLI AUTORI}

I lavori pubblicati sui Quaderni ITJM non verranno indicizzati, ma saranno liberamente disponibili in un'apposita sezione del sito FADOI (http://www.fadoi.org/) e della rivista ufficiale.

Gli Autori i cui lavori siano accettati per la pubblicazione sui Quaderni ITJM e che fossero interessati a vederli pubblicati anche sulla rivista ufficiale, dovranno sottomettere attraverso il sito dell'ITJM (www.italjmed.org) la versione (già tradotta) in inglese e redatta in modo conforme alle linee guida della rivista; seguiranno poi la procedura di selezione tramite peer review e, se accettati, saranno inseriti nel piano editoriale standard.

\section{STAFF EDITORIALE}

Paola Granata, Journal Manager

paola.granata@pagepress.org

Claudia Castellano, Production Editor

Tiziano Taccini, Technical Support
PUBBLICATO DA

PAGEPress Publications

via A. Cavagna Sangiuliani 5

27100 Pavia, Italy

T. +39.0382 .1549020$

F: +39.0382 .1727454$

\section{pagepress}

www.pagepress.org info@pagepress.org

\section{QUADERNI - ITALIAN JOURNAL OF MEDICINE}

Tutti gli articoli pubblicati sui QUADERNI - Italian Journal of Medicine sono redatti sotto la responsabilità degli Autori. La pubblicazione o la ristampa degli articoli della rivista deve essere autorizzata per iscritto dall'editore. Ai sensi dell'art. 13 del D.Lgs 196/03, i dati di tutti i lettori saranno trattati sia manualmente, sia con strumenti informatici e saranno utilizzati per l'invio di questa e di altre pubblicazioni e di materiale informativo e promozionale. Le modalità di trattamento saranno conformi a quanto previsto dall'art. 11 del D.Lgs 196/03. I dati potranno essere comunicati a soggetti con i quali PAGEPress intrattiene rapporti contrattuali necessari per l'invio delle copie della rivista. Il titolare del trattamento dei dati è PAGEPress Srl, via A. Cavagna Sangiuliani $5-27100$ Pavia, al quale il lettore si potrà rivolgere per chiedere l'aggiornamento, l'integrazione, la cancellazione e ogni altra operazione di cui all'art. 7 del D.Lgs 196/03.

https://www.italjmed.org/index.php/ijm/quad 
